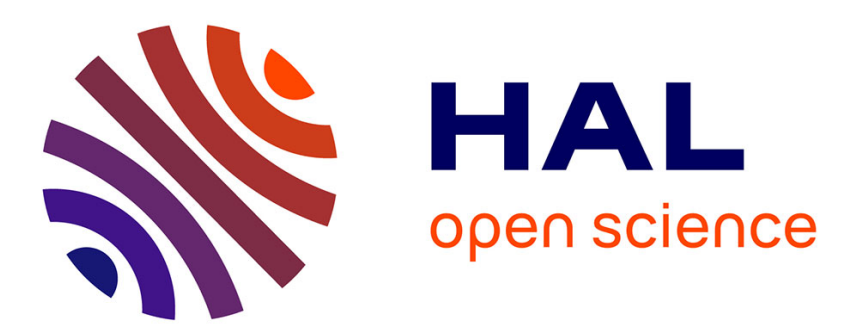

\title{
Orbifoldes géométriques spéciales et classification biméromorphe des variétés kählériennes compactes
}

Fédéric Campana

\section{To cite this version:}

Fédéric Campana. Orbifoldes géométriques spéciales et classification biméromorphe des variétés kählériennes compactes. 2009. hal-00356763

\section{HAL Id: hal-00356763 \\ https://hal.science/hal-00356763}

Preprint submitted on 28 Jan 2009

HAL is a multi-disciplinary open access archive for the deposit and dissemination of scientific research documents, whether they are published or not. The documents may come from teaching and research institutions in France or abroad, or from public or private research centers.
L'archive ouverte pluridisciplinaire HAL, est destinée au dépôt et à la diffusion de documents scientifiques de niveau recherche, publiés ou non, émanant des établissements d'enseignement et de recherche français ou étrangers, des laboratoires publics ou privés. 


\section{ORBIFOLDES GÉOMÉTRIQUES SPÉCIALES ET CLASSIFICATION BIMÉROMORPHE DES VARIÉTÉS KÄHLÉRIENNES COMPACTES}

Frédéric Campana

4 décembre 2008

\section{Table des matières}

1 INTRODUCTION 4

1.1 Abstract ....................... 4

1.2 Introduction . . . . . . . . . . . . . . . 5

2 LA CATÉGORIE DES ORBIFOLDES GÉOMÉTRIQUES 8

2.1 Diviseurs orbifoldes . . . . . . . . . . . . . 8

2.2 Invariants : dimension canonique, groupe fondamental, points entiers. . . . . . . . . . . . . . . 10

2.3 Morphismes orbifoldes. . . . . . . . . . . . . . . . 11

2.4 Résolution d'une orbifolde géométrique. . . . . . . . . . . . . . 15

2.5 Faisceaux de formes différentielles sur les orbifoldes géométriques lisses. . . . . . . . . . . . . . . . 15

2.6 Tenseurs holomorphes orbifoldes. . . . . . . . . . . . . . 18

2.7 Dimension canonique d'un faisceau différentiel de rang 1 . . . 19

2.8 Invariance biméromorphe de la dimension canonique . . . . . . 21

2.9 Invariance étale de la dimension canonique . . . . . . . . . . . . . 22

2.10 Inégalité de Bogomolov (version orbifolde) . . . . . . . . . . . 23 
2.11 Orbifoldes géométriques log-canoniques et klt . . . . . . . . . 24

2.12 Équivalence biméromorphe. . . . . . . . . . . . . . . . . . . . 25

2.13 Restriction à une sous-variété. . . . . . . . . . . . . . . . . . 28

3 BASE ET FIBRE ORBIFOLDES D'UN MORPHISME 31

3.1 Base orbifolde d'un morphisme . . . . . . . . . . . 31

3.2 Fibrations nettes. . . . . . . . . . . . . . . . . 34

3.3 Composition de fibrations . . . . . . . . . . . . . . . 36

3.4 Fibres orbifoldes. . . . . . . . . . . . . . . . . 38

4 DIMENSION CANONIQUE D'UNE FIBRATION. 40

4.1 Dimension canonique d'une fibration. . . . . . . . . . . . 40

4.2 Équivalence biméromorphe de fibrations. . . . . . . . . . . . . 42

4.3 Fibration de Moishezon-Iitaka. . . . . . . . . . . . . . . . . 45

4.4 Fibrations de type général et orbifoldes spéciales. . . . . . . . 46

5 COURBES RATIONNELLES ORBIFOLDES. 47

5.1 Notion de courbe rationnelle orbifolde . . . . . . . . . . . . 47

5.2 "Descente" de courbes rationnelles. . . . . . . . . . . . . . 50

5.3 Lemme de "cassage" orbifolde. . . . . . . . . . . . . . . . . 51

5.4 Semi-positivité générique. . . . . . . . . . . . . . . 53

5.5 Quotients globaux et relèvement de courbes rationnelles orbifoldes. . . . . . . . . . . . . . . . . 5 54

5.6 Uniréglage, Connexité Rationnelle orbifolde . . . . . . . . . 56

5.7 Quotients rationnels orbifoldes . . . . . . . . . . . . . 59

5.8 Le cas des quotients globaux. . . . . . . . . . . . . . 61

5.9 Sections orbifoldes. . . . . . . . . . . . . . 63

5.10 Quotients globaux : l'implication $R E \Longrightarrow R C C$. . . . . . . . 68

5.11 Uniréglage et Dimension Canonique . . . . . . . . . . . . . . 70

5.12 Appendice : Quotients méromorphes. . . . . . . . . . . . 72

6 ADDITIVITÉ ORBIFOLDE $\quad 74$

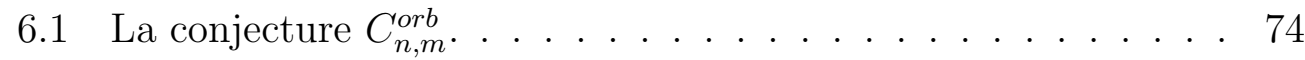

6.2 Le cas des fibrations de type général. . . . . . . . . . . . . . . 74

6.3 Première application $: \kappa=0 \ldots \ldots . \ldots . \ldots 76$

6.4 Le quotient $\kappa$-rationnel (conditionnel) . . . . . . . . . 77 
7 FIBRATIONS DE TYPE GÉNÉRAL. 80

7.1 Fibrations de type général : définition, stabilité par composition 80

7.2 Faisceaux de Bogomolov et fibrations de type général. . . . . . 81

7.3 Restriction à une sous-variété générique. . . . . . . . . . . . . 81

7.4 Presque-holomorphie des fibrations de type général . . . . . . 84

7.5 Réduction de type général simultanée. . . . . . . . . . . . . 86

8 ORBIFOLDES GÉOMÉTRIQUES SPÉCIALES 88

8.1 Fibre et base orbifolde d'une fibration . . . . . . . . . . . 88

8.2 Premiers exemples . . . . . . . . . . . . . . . . . . 89

8.3 Composition de fibrations spéciales . . . . . . . . . . . . 90

9 LE “COEUR” D'UNE ORBIFOLDE GÉOMÉTRIQUE 92

9.1 Construction du Coeur. . . . . . . . . . . . . . . . . . 92

9.2 Fonctorialité. . . . . . . . . . . . . . . . . . . . . . 94

9.3 Connexité par chaînes spéciales. . . . . . . . . . . . . . . . 94

9.4 Invariance par revêtement étale. . . . . . . . . . . . . . . 95

10 DÉCOMPOSITION DU COEUR. $\quad 97$

10.1 La décomposition conditionnelle du coeur. . . . . . . . . . . . 97

10.2 Existence de variétés de type et dimension donnés. . . . . . . 99

10.3 Analogie avec les algèbres de Lie. . . . . . . . . . . . . . . . . 101

10.4 Fonctorialité (conditionnelle) . . . . . . . . . . . . . . . . . 101

10.5 Relèvement de propriétés par dévissage. . . . . . . . . . . . . . 102

11 GROUPE FONDAMENTAL 104

11.1 Groupe fondamental d'une orbifolde géométrique lisse . . . . . 104

11.2 Suite exacte associée à une fibration orbifolde nette . . . . . . 107

11.3 Revêtement universel d'une orbifolde géométrique lisse . . . . 110

11.4 Finitude résiduelle et critère d'abélianité. . . . . . . . . . . . . 112

11.5 Г-réduction (ou réduction de Shafarevich) orbifolde. . . . . . . 115

12 CONJECTURES $\quad 119$

12.1 Stabilité par déformation et spécialisation . . . . . . . . . . 119

12.2 Groupe fondamental et revêtement universel . . . . . . . . . . 121

12.3 Pseudométrique de Kobayashi . . . . . . . . . . . . . . . . . . 123

12.4 Points rationnels : corps de fonctions . . . . . . . . . . . . . . 124

12.5 Points rationnels : arithmétique . . . . . . . . . 126 
12.6 Familles de variétés canoniquement polarisées. . . . . . . . . . 128

12.7 Formes différentielles . . . . . . . . . . . . . . . . . . 131

\section{INTRODUCTION}

\subsection{Abstract}

This is the geometric-orbifold version of [Ca01/04]. We define the bimeromorphic category of geometric orbifolds. These interpolate between (compact Kähler) manifolds and such manifolds with logarithmic structure, and may be considered as "virtual" ramified covers of the underlying manifold. These geometric orbifolds are here considered as fully geometric objects, and thus come naturally equipped with the usual invariants of varieties : morphisms and bimeromorphic maps, differential forms, fundamental groups and universal covers, fields of definition and rational points. The general expectation is that their geometry is qualitatively the same as that of manifolds with similar invariants. The most elementary of such geometric properties are established here. The arguments of [Ca01] can then be directly adapted to extend the main structure results established there to this geometric-orbifold category. We hope to come back to deeper aspects later. The motivation is that the natural frame for the theory of bimeromorphic classification of compact Kähler (and complex projective) manifolds without orbifold structure unavoidably seems to be the category of geometric orbifolds, as shown here (and in [Ca01] for manifolds) by the fonctorial decomposition of special orbifolds as tower of orbifolds with fibres having either $\kappa_{+}=-\infty$ or $\kappa=0$, and also, in a different context, by the minimal model program, in which most proofs naturally work only after the adjunction of a "boundary". An orbifold is special if it does not map "stably" onto a (positive-dimensional) orbifold of general type, while having $\kappa_{+}=-\infty$ means that it maps only onto orbifolds having $\kappa=-\infty$, and is expected to mean rationally connected in the orbifold category.

Moreover, fibrations enjoy in the bimeromorphic category of geometric orbifolds extension (or "additivity") properties not satisfied in the category of varieties without orbifold structure, permitting to express invariants of the total space as the extension (or "sum") of those of the generic fibre and of 
the base. Indeed, the discrepancy between the invariants of the total space and the "sum" of those of the base and general fibres is mainly due to the presence of multiple fibres, taken into account in the orbifold (but not in the manifold) category. For example, the natural sequence of fundamental groups always becomes exact in the orbifold category. Also the total space of a fibration is special if so are the generic fibre and the base. Both properties are very false without orbifold structures. This makes this category suitable to lift properties from orbifolds having either $\kappa_{+}=-\infty$ or $\kappa=0$ to those which are special. And even leads to expect that specialness is the exact geometric characterisation of some important properties (such as potential density or vanishing of the Kobayashi pseudometric).

Let us notice that the notion of morphism used to treat fundamental groups, based on the classical divisibility differs, by necessity, from the one used to deal with other geometrical aspects.

\subsection{Introduction}

L'objectif du texte est en priorité de définir et d'établir les propriétés de base de nouveaux objets (les "orbifoldes géométriques") qui semblent être essentiels pour la compréhension de la structure birationnelle des variétés projectives ou Kählériennes compactes, et en donnent une vue synthétique globale très simple. Les démonstrations données reposent cependant sur les techniques usuelles de la géométrie algébrique/analytique. De nombreuses questions ou conjectures à leur sujet sont également formulées.

Le présent texte est la suite de [Ca01/04]. Dans ce texte était introduite la notion de base orbifolde $(Y \mid \Delta(f))$ d'une fibration $f: X \rightarrow Y$, avec $X$ compacte Kähler, cette base orbifolde étant vue comme un revêtement ramifié "virtuel" de $Y$ éliminant virtuellement les fibres multiples en codimension 1 de $f$. Ces fibres multiples forment l'obstruction principale à exprimer les invariants géométriques fondamentaux de $X$ comme extension (ou "somme") de ceux de la fibre générique $X_{y}$, et de la base $Y$ de $f$. On y avait introduit, de plus, une nouvelle classe de variétés, dites spéciales. Ce sont, par définition, celles n'ayant pas de fibration sur une base orbifolde de type général. Un $X$ arbitraire y était scindé, à l'aide d'une unique fibration fonctorielle (son "coeur"), en ses parties antithétiques : spéciale (les fibres) et de type général (la base orbifolde). On y avait décomposé ${ }^{1}$ fonctoriellement toute

\footnotetext{
${ }^{1}$ Conditionnellement en une version orbifolde $C_{n, m}^{o r b}$ de la conjecture $C_{n, m}$ d'Titaka.
} 
variété spéciale en tours de fibrations à fibres orbifoldes ayant soit $\kappa_{+}=-\infty$ (version conjecturale de la connexité rationnelle), soit $\kappa=0$. La géométrie spéciale apparaissant ainsi comme la combinaison au sens orbifolde de ces deux géométries classiques.

Cette décomposition (et peut-être aussi, parallèlement, la théorie des modèles minimaux où l'adjonction d'un "bord" joue un rôle crucial dans nombre de démonstrations) semble indiquer que le cadre naturel de la théorie de la classification des variétés (projectives complexes ou Kählériennes compactes) est la catégorie biméromorphe des orbifoldes géométriques. Cette catégorie restant, dans une première étape, à définir.

L'objectif principal du présent texte est de le faire (du moins dans le cas "lisse"), et d'en développer les propriétés de base les plus simples (celles dont la démonstration s'adapte directement du cas des variétés à celle des orbifoldes). Le point de vue adopté est que ces objets, qui interpolent entre les variétés sans structure orbifolde et les variétés logarithmiques, sont des objets géométriques à part entière, et sont donc équipés de tous les attributs des variétés usuelles : morphismes, transformations biméromorphes, formes différentielles, groupe fondamental et revêtement universel, corps de définition, points rationnels. Nous espérons développer des aspects plus profonds ultérieurement. En particulier, seules les orbifoldes lisses sont étudiées ici. Il semble indispensable de considérer un cadre plus général (celui des orbifoldes "klt" ou "l.c") pour les développements ultérieurs.

Notons que les fibrations possèdent bien, dans cette catégorie, les propriétés attendues d'additivité qui font défaut dans celle des variétés sans structure orbifolde, et qui sont à l'origine de leur introduction. Par exemple, la suite des groupes fondamentaux devient exacte dans cette catégorie. De même, l'espace total d'une fibration à fibres et base orbifoldes spéciales est spéciale. Cette catégorie devrait ainsi permettre, par dévissage, de "relever" à la "géométrie spéciale" certaines des propriétés attendues des "géométries $\kappa_{+}=-\infty$ et $\kappa=0$ ". Et même de conjecturer que certaines propriétés importantes (densité potentielle et nullité de la pseudométrique de Kobayashi) sont exactement caractéristiques des orbifoldes spéciales.

Le contenu du texte est le suivant : le $\S 2$ introduit la catégorie des orbifoldes, les morphismes étant définis de 3 façons différentes (équivalentes) : multiplicités (voie géométrique; voir définition 2.3, qui est probablement la contribution principale du présent texte), préservation des faisceaux de formes différentielles, et disques testants. Le $\S 3$ définit la base orbifolde "stable" d'une fibration $f:(X \mid \Delta) \rightarrow Y$, et calcule la base orbifolde "stable" 
d'une composée. Dans le $\S 4$, on établit l'invariance biméromorphe de la dimension de Kodaira de la base orbifolde stable d'une fibration, résultat utilisé constamment dans la suite. Le $§ 5$ définit les courbes rationnelles orbifoldes, et pose la question de savoir si leurs propriétś sont analogues à celles du cas non orbifolde. On montre que c'est bien le cas lorsque l'orbifolde géométrique considérée est un "quotient global" au sens de 5.15. La considération du champ algébrique associé pourrait permettre de traiter le cas général avec des arguments similaires si les multiplicités sont entières. Le $\S 6$ contient des rappels extraits de [Ca01] sur l'additivité de la dimension de Kodaira dans le cadre orbifolde. C'est le résultat technique central du présent texte. Le $\S 7$ contient des préliminaires techniques sur les fibrations de type général, nécessaires pour la construction du coeur, effectuée au $\S 9$, après un exposé au $\S 8$ des propriétés et exemples de base des orbifoldes spéciales. Au $\S 10$, on décompose (conditionnellement en $C_{n, m}^{\text {orb }}$ ) fonctoriellement le coeur en tour de fibrations à fibres orbifoldes ayant soit $\kappa_{+}=-\infty$, soit $\kappa=0$. Ce dévissage permet de formuler des conditions sous lesquelles "relever" aux orbifoldes spéciales les propriétés attendues des orbifoldes ayant soit $\kappa_{+}=-\infty$, soit $\kappa=0$. Dans le $\S 11$, on définit et étudie le groupe fondamental et le revêtement universel, et on construit la $\Gamma$-réduction dans le cadre orbifolde (avec morphismes au sens divisible). Dans le $\S 12$, on énonce des conjectures qui étendent directement au cadre orbifolde celles de [Ca01], et résultent du "relèvement" du $\S 10$.

Le projet d'étendre aux orbifoldes géométriques les résultats de [Ca01] y était déjà proposé. Il a été aussitôt entrepris par S. Lu dans math.AG/0211029. La définition d'un morphisme orbifolde qui y est donnée, en terme de "relevant multiplicities", permet d'adapter directement les arguments de [Ca01], mais n'admet pas d'interprétation géométrique explicite, indispensable pour les applications et développements ultérieurs.

Je voudrais remercier D. Greb et K. Jabusch pour m'avoir signalé une erreur dans la version initiale. 


\section{LA CATÉGORIE DES ORBIFOLDES GÉOMÉTRIQUES}

\subsection{Diviseurs orbifoldes}

Definition 2.1 Soit $X$ un espace analytique complexe normal (dénombrable à l'infini; X sera, de plus, supposé compact et connexe dans la suite de ce texte, à l'exception du présent §2 essentiellement). On note $W(X)$ l'ensemble des diviseurs de Weil irréductibles de $X$.

Une multiplicité orbifolde sur $X$ est une application $m: W(X) \rightarrow$ $(\mathbb{Q} \cup+\infty):=\overline{\mathbb{Q}^{+}}$telle que $m(D) \geq 1$ pour tout $D \in W(X)$, et telle que $m(D)=1$ pour localement presque tout $D \in W(X)$ (ie : pour tout compact $K$ de $X, m(D)=1$ pour tous les $D \in W(X)$ rencontrant $K$, sauf un nombre fini d'entre eux). Une telle multiplicité orbifolde est dite entière si elle prend ses valeurs dans $(\mathbb{N} \cup+\infty)$.

Une multiplicité orbifolde $m$ sera dite finie si elle ne prend pas la valeur $+\infty$.

Le diviseur orbifolde associé est $\Delta:=\sum_{D \in W(X)}(1-1 / m(D)) . D$. C'est une somme localement finie définissant un $\mathbb{Q}$-diviseur effectif sur $X$. (Par convention, $1 /(+\infty):=0)$. On notera $m_{\Delta}: W(X) \rightarrow(\mathbb{Q} \cup+\infty)$ la multiplicité orbifolde définissant $\Delta$.

Le support de $\Delta$, noté $\operatorname{supp}(\Delta)$, ou $\lceil\Delta\rceil$, est la réunion des $D \in W(X)$ tels que $m(D)>1$.

Une orbifolde géométrique ${ }^{2}$ est un couple, noté $(X \mid \Delta)$, de la forme précédente. Une orbifolde géométrique $(X \mid \Delta)$ sera dite finie si sa multiplicité $m_{\Delta}$ est finie, et entière si sa multiplicité l'est.

On dira que $(X \mid \Delta)$ est lisse si $X$ est lisse et si $\operatorname{supp}(\Delta)$ est un diviseur $\grave{a}$ croisements normaux.

Remarque 2.2 1. Lorsque $m$ ne prend que les valeurs 1 et $+\infty$, le diviseur $\Delta$ est entier, réduit, de multiplicité 1 . On dira que l'orbifolde géométrique

\footnotetext{
${ }^{2}$ Le terme "géométrique" provient de ce que l'on ne conserve de la structure orbifolde que le support (un diviseur) et les multiplicités, mais que l'on ne se donne pas d'action locale d'un groupe. En ce sens, une "orbifolde géométrique" peut être vue comme la trace géométrique en codimension 1 d'une orbifolde au sens usuel. Nous espérons que ce terme ne créera as de confusion avec les orbifoldes usuelles.
} 
$(X \mid \Delta)$ est ouverte, ou : logarithmique. On notera $(X \mid D)$ une telle orbifolde géométrique. Le cas général interpole donc entre les cas propre (où $\Delta=0$ ) et logarithmique.

Toute variété lisse et quasi-projective $U$ s'écrit : $U=X-D$, avec $(X \mid D)$ lisse. Les propriétés de $U$ sont celles de $(X \mid D)$ ne dépendant que de $X-D$. Ce sont en fait les propriétés "birationnelles" de $(X \mid D)$ au sens défini ci-dessous. Nous en verrons de nombreuses, nouvelles.

On écrira aussi $\Delta=\sum_{j \in J}\left(1-1 / m_{j}\right) \cdot D_{j}$ si $J$ est un sous-ensemble localement fini de $W(X)$ contenant tous les $D$ tels que $m(D)>1$, avec $m_{j}=m\left(D_{j}\right), \forall j \in J$.

2. On peut considérer, plus géneralement, des fonctions de multiplicité à valeurs réelles dans $\{[1,+\infty[\cup\{+\infty\}\}$. Les définitions et résultats ci-dessous s'appliquent, ainsi que leurs démonstrations, avec des modifications mineures, à ce cadre élargi (susceptible d'autres applications).

3. Si $\Delta, \Delta^{\prime}$ sont des diviseurs orbifoldes sur $X$, de fonctions de multiplicité $m, m^{\prime}$ respectivement, on dira que $\Delta \geq \Delta^{\prime}$ si $m \geq m^{\prime}$. (Ceci signifie en effet que $\left(\Delta-\Delta^{\prime}\right)$ est un diviseur effectif). Si ces deux orbifoldes géométriques sont entières, on dira que $\Delta^{\prime}$ divise $\Delta$ (noté $m_{\Delta^{\prime}}(D) \mid m_{\Delta^{\prime}}(D)$ divise $m_{\Delta}(D), \forall D \in$ $W(X)$ (avec la convention : $n$ divise $+\infty, \forall n>0$, entier).

4. On définit de manière évidente le produit de deux orbifoldes géométriques. Ce produit est donc lisse si les facteurs le sont.

5. Si $\Delta, \Delta^{\prime}$ sont deux (ou même une famille d') orbifoldes géométriques sur $X$ on définit de manière évidente $\sup \left\{\Delta, \Delta^{\prime}\right\}$ et inf $\left\{\Delta, \Delta^{\prime}\right\}$.

6. D. Abramovich a introduit, dans [Abr 07], le terme de constellation et pour son raffinement toroidal, qui lui est dû, celui de firmament. La notion de "constellation" consiste en la donnée d'un système (compatible) de diviseurs orbifoldes géométriques sur toutes les modifications propres de $X$, considérées simultanément. Nous tentons ici de considérer les propriétés biméromorphes (définies de manière adéquate) des "orbifoldes géométriques" individuelles, sans inclure la totalité de la constellation associée dans la donnée initiale. La différence entre les deux notions semble cependant inessentielle.

D'ailleurs, dans le cas considéré ici, où $X$ est projective lisse et le support de $\Delta$ à croisement normaux, la notion d'orbifolde géométrique est un cas particulier de celle de "champ algébrique" lisse de Deligne-Mumford, et le terme est donc compatible (au niveau des objets, sinon des morphismes définis ci-dessous) avec les terminologies antérieures.

L' expression "orbifolde géométrique" sera parfois abrégée en "orbifolde" 
dans la suite. On parlera ainsi de "morphismes orbifoldes, fibre ou base orbifolde" d'une fibration. Ceci ne devrait pas créer de confusion, puisque les "orbifoldes" au sens usuel ne sont jamais considérées dans le présent texte.

\subsection{Invariants : dimension canonique, groupe fonda- mental, points entiers.}

Le principe que nous voudrions illustrer dans le texte qui suit est le suivant :

1. Les orbifoldes géométriques (lisses) sont des objets géométriques à part entière, au même titre que les variétés complexes (projectives ou compactes) : on peut leur attribuer en particulier les invariants géomériques définis cidessous.

2. Leurs propriétés géométriques sont les mêmes que celles des variétés (sans structure orbifolde géométrique) ayant des invariants analogues.

3. Nombre de ces propriétés sont établies en adaptant (en général sans difficultés majeures) les démonstrations établissant celles des variétés sans structure orbifolde géométrique. Pour certaines propriétés cependant, l'adaptation semble requérir des idées nouvelles. Des exemples sont : les courbes rationnelles orbifoldes, ou le groupe fondamental des orbifoldes géométriques qui sont soit Fano, soit à fibré canonique trivial.

- Si $(X \mid \Delta)$ est une orbifolde géométrique, on définit (voir [Ca04], les définitions détaillées sont aussi données ci-dessous, dans les chapitres correspondants ) :

1. Son fibré canonique $K_{X \mid \Delta}:=K_{X}+\Delta$ : c'est un $\mathbb{Q}$-diviseur sur $X$.

2. Sa dimension canonique (ou de Kodaira $\left.{ }^{3}\right): \kappa(X \mid \Delta):=\kappa\left(X, K_{X}+\Delta\right) \geq$ $\kappa(X) \in\{-\infty, 0,1, \ldots, \operatorname{dim}(X)\}$, lorsque $X$ est compacte et irréductible.

On dira que $(X \mid \Delta)$ est de type général si $\kappa(X \mid \Delta)=\operatorname{dim}(X)>0$.

3. On définira aussi des faisceaux de formes (pluri)-différentielles et, plus généralement, de tenseurs holomorphes sur une orbifolde géométrique lisse.

\footnotetext{
${ }^{3}$ Habituellement appelée "dimension de Kodaira", elle est en fait introduite pour les surfaces dans le livre de Shafarevitch et al. sur la classification des surfaces projectives, et en général par S. Iitaka et B. Moishezon (dont nous suivons la terminologie). K. Kodaira ne l'a utilisée qu'en 1975, alors qu'elle était déjà d'usage courant.
} 
(Voir $\S 2.5$ et $\S 2.6$ ci-dessous).

- Lorsque $(X \mid \Delta)$ est entière, on peut aussi définir :

4. Son groupe fondamental $\pi_{1}(X \mid \Delta)$ et son revêtement universel (voir [Ca04] et le $\S 11$ ci-dessous).

5. Sa pseudométrique de Kobayashi $d_{X \mid \Delta}$ (voir [Ca04] et le $\S 12.3$ cidessous).

6. Ses points entiers sur un corps de nombres, pour un modèle donné. (Voir [Ca05], et le $\$ 12.5$ ci-dessous).

7. La notion d'orbifolde géométrique peut naturellement être définie en géométrie algébrique sur d'autres corps que $\mathbb{C}$. Un cas intéressant est celui des corps de fonctions (méromorphes sur une courbe projective définie sur $\mathbb{C}$ ou sur un corps fini). Voir [Ca01] ou le $\S 12.4$ ci-dessous.

\subsection{Morphismes orbifoldes.}

La définition suivante est la contribution principale du présent texte :

Definition 2.3 Soit $f: Y \rightarrow X$ une application holomorphe entre espaces analytiques complexes normaux, et $\Delta_{Y}, \Delta_{X}$ des diviseurs orbifoldes sur $Y, X$ respectivement. On note ici $m_{Y}, m_{X}$ les multiplicités orbifoldes associées. On dit que $f$ induit un morphisme orbifolde (noté alors $f:\left(Y \mid \Delta_{Y}\right) \rightarrow$ $\left.\left(X \mid \Delta_{X}\right)\right)$ si :

1. $f(Y)$ n'est pas contenu dans $\left|\Delta_{X}\right|$.

2. $X$ est $\mathbb{Q}$-factorielle au sens algébrique (ie : tout diviseur de Weil irréductible sur $X$ est $\mathbb{Q}$-Cartier $)$.

3. pour tout $D \in W(X)$, et tout $E \in W(Y)$, on a : t. $m_{Y}(E) \geq m_{X}(D)$, si $t>0$, où $: t=t_{E, D} \in \mathbb{Q}^{+}$est tel que $f^{*}(D)=t_{E, D} \cdot E+R$, avec $R$ un diviseur effectif de $Y$ ne contenant pas $E$.

Definition 2.4 Dans l'étude du groupe fondamental orbifolde (défini seulement pour les orbifoldes géométriques entières, voir §11), nous utiliserons une notion plus restrictive de morphisme orbifolde, celle de morphisme orbifolde divisible : il s'agit d'un morphisme orbifolde dans le sens précédent, mais satisfaisant la variante "muliplicités classiques" : $m_{X}(D)$ divise $t . m_{Y}(E)^{4}$, pour tous $D, E$ comme dans la condition 2 ci-dessus. (Par convention, $+\infty$ est multiple de tout entier $m>0$ ).

\footnotetext{
${ }^{4} \mathrm{Au}$ lieu de $: t \cdot m_{Y}(E) \geq m_{X}(D)$.
} 
Pour distinguer ces deux types de morphismes, nous appellerons "morphismes non-classiques" ceux définis en 2.3 ci-dessus, et "morphismes classiques, ou divisibles" ceux définis ici.

On notera $G_{e o r b}{ }^{Q}$ la catégorie des orbifoldes géométriques $\mathbb{Q}$-factorielles munie des morphismes non-classiques, et Georb ${ }^{Z}$ sa sous-catégorie pleine constituée des orbifoldes entières. On notera en fin Georb div la catégorie des orbifoldes géométriques $\mathbb{Q}$-factorielles entières munie des morphismes "classiques" (ou "divisibles").

Pour indiquer qu'un morphisme orbifolde $f:\left(Y \mid \Delta_{Y}\right) \rightarrow\left(X \mid \Delta_{X}\right)$ appartient à l'une de ces trois catégories, on le notera : $f:\left(Y \mid \Delta_{Y}\right)^{*} \rightarrow\left(X \mid \Delta_{X}\right)^{*}$, avec $*=\operatorname{div}, Z, Q$ selon le cas.

\section{Remarque 2.5}

0. Les motivations pour la condition 3. de la définition 2.3 précédente sont multiples, bien que peu évidentes a priori : tout d'abord, cette condition est exactement celle qui préserve les faisceaux de formes pluri-différentielles (voir proposition 2.12), ainsi que les morphismes du disque unité pour les orbifoldes géométriques entières (voir proposition 2.7).

Enfin, une motivation géométrique plus directe (dans le cas divisible) est la compatibilité avec la composition des fibrations : soient $f: X \rightarrow Y$ et $g: Y \rightarrow Z$ deux fibrations entre variétés complexes projectives normales. On suppose $f$ et $g$ à fibres équidimensionnelles pour simplifier.

Soit $D$ un diviseur de Weil sur $Z$. Alors la multiplicité de la fibre de $(g \circ f)$ au-dessus du point générique de $D$ est le $p g c d$, noté $\mu(D)$, des $t_{j} \cdot m_{j}$ si $g^{*}(D)=$ $\sum_{j} t_{j} .\left(E_{j}\right)$, où $m_{j}=\operatorname{pgcd}\left(s_{i j}\right)$, avec : $f^{*}\left(E_{j}\right)=\sum_{i} s_{i j} . F_{i j}$. (Les entiers $t_{j}, s_{i j}$ sont bien définis au-dessus des points génériques de $D, E_{j}$, puisque $f, g$ sont à fibres équidimensionnelles, de sorte que tous les $E_{j}$ ont pour image $D$ ). Les $m_{j}$ sont donc simplement les multiplicités usuelles des fibres de $f$ au-dessus des points génériques des $E_{j}$.

Si l'on définit une orbifolde géométrique $\left(Y \mid \Delta_{f}\right)$ sur $Y$ en posant : $m_{\Delta_{f}}\left(E_{j}\right):=m_{j}$ pour tout diviseur de Weil $E_{j}$ sur $Y$ comme ci-dessus, et une orbifolde géométrique $\left(Z \mid \Delta_{g \circ f}\right)$ en posant $m_{\Delta_{g \circ f}}(D):=\mu(E)$, pour tout $E \subset Z$, alors : pour tout diviseur orbifolde $\Delta_{Y}$ sur $Y$, l'application $g:\left(Y \mid \Delta_{Y}\right) \rightarrow\left(Z \mid \Delta_{g \circ f}\right)$ est un morphisme orbifolde (au sens de 2.3) si et seulement si $\Delta_{Y} \geq \Delta_{f}$.

Cette situation sera étudiée en détail aux $\S 3.1$ et $\S 3.3$.

1. Si $m_{X}(D)=1$ et si $X$ est factorielle, la condition 2. est vide (puisque $t>0$ est entier et $m_{Y}(E) \geq 1$ ). Seuls les $D \subset\left\lceil\Delta_{X}\right\rceil$ et $E$ tels que $f(E) \subset$ 
$\left\lceil\Delta_{X}\right\rceil$ fournissent donc des conditions non vides. Si $X$ et $Y$ sont compacts, les conditions à vérifier sont donc en nombre fini.

2. Si $f:\left(Y \mid \Delta_{Y}\right) \rightarrow\left(X \mid \Delta_{X}\right)$ et $g:\left(Z \mid \Delta_{Z}\right) \rightarrow\left(Y \mid \Delta_{Y}\right)$ sont des morphismes, le composé $f \circ g$ aussi. La démonstration est immédiate.

3. Si $\Delta_{X}=\Delta_{Y}=0$, toute application holomorphe $f: X \rightarrow Y$ est un morphisme orbifolde. Si $f:\left(Y \mid \Delta_{Y}\right) \rightarrow\left(X \mid \Delta_{X}\right)$ est un morphisme orbifolde, et si $\Delta_{Y}^{+} \geq \Delta_{Y}$, alors $f:\left(Y \mid \Delta_{Y}^{+}\right) \rightarrow\left(X \mid \Delta_{X}\right)$ est un morphisme orbifolde. De même : si $\Delta_{X} \geq \Delta_{X}^{-}$, alors $f:\left(Y \mid \Delta_{Y}\right) \rightarrow\left(X \mid \Delta_{X}^{-}\right)$est un morphisme orbifolde.

4. Si $\left(X \mid \Delta_{X}\right)$ est logarithmique, alors $f:\left(Y \mid \Delta_{Y}\right) \rightarrow\left(X \mid \Delta_{X}\right)$ est un morphisme orbifolde si et seulement si $\Delta_{Y} \geq f^{-1}\left(X \mid \Delta_{X}\right)$.

5. On dit que $f:\left(Y \mid \Delta_{Y}\right) \rightarrow\left(X \mid \Delta_{X}\right)$ est un morphisme orbifolde biméromorphe élémentaire (ou une modification orbifolde élémentaire) si c'est un morphisme orbifolde, si $f: Y \rightarrow X$ est birationnel, et si $f_{*}\left(\Delta_{Y}\right)=\Delta_{X}$. On suppose ici que les deux espaces $Y, X$ considérés sont algébriquement $\mathbb{Q}$-factoriels. Noter que les trois propriétés sont indépendantes (deux d'entre elles n'impliquent pas la troisième). On dira que deux telles orbifoldes géométriques sont biméromorphiquement équivalentes s'il existe une chaine de morphismes orbifoldes biméromorphes élémentaires les reliant. De manière équivalente : c'est la relation d'équivalence engendrée par les morphismes orbifolde biméromorphes élémentaires.

- Voir 2.32 et 2.33 pour des exemples utiles d'équivalences biméromorphes.

6. Si $f:\left(Y \mid \Delta_{Y}\right) \rightarrow(X \mid \Delta)$ et $f^{\prime}:\left(Y \mid \Delta_{Y}\right) \rightarrow\left(X \mid \Delta^{\prime}\right)$ sont des morphismes orbifoldes, ils se factorisent par $f^{+}:\left(Y \mid \Delta_{Y}\right) \rightarrow\left(X \mid \Delta^{+}\right)$, avec $\Delta^{+}:=$ $\sup \left\{\Delta, \Delta^{\prime}\right\}$. De même, si $f:(X \mid \Delta) \rightarrow\left(Y \mid \Delta_{Y}\right)$ et $f:\left(X \mid \Delta^{\prime}\right) \rightarrow\left(Y \mid \Delta_{Y}\right)$ sont des morphismes orbifoldes, ils se factorisent par $f^{-}:\left(X \mid \Delta^{-}\right) \rightarrow\left(Y \mid \Delta_{Y}\right)$, avec $\Delta^{-}:=\inf \left\{\Delta, \Delta^{\prime}\right\}$.

7. Si $f: Y \rightarrow\left(X \mid \Delta_{X}\right)$ est une application holomorphe propre et surjective avec $Y$ et $\left(X \mid \Delta_{X}\right)$ lisses, et $f^{-1}\left(\left\lceil\Delta_{X}\right\rceil\right)$ à croisement normaux, il existe un élément minimum, noté $f^{+}\left(\Delta_{X}\right)$, parmi les diviseurs orbifoldes $\Delta_{Y}$ sur $Y$ tels que $f:\left(Y \mid \Delta_{Y}\right) \rightarrow\left(X \mid \Delta_{X}\right)$ soit un morphisme orbifolde. On l'appelle relèvement de $\Delta_{X}$ à $Y$. Pour tout $E \in W(Y)$, la $f^{+}\left(\Delta_{X}\right)$-multiplicité de $E$ est $m_{Y}(E):=\sup \left\{1, \sup _{D}\left\{m(D) / t_{E, D}\right\}\right\}, D \in W(X)$ tel que $f^{*}(D)=$ $t_{E, D} \cdot E+R$, avec $t_{E, D}>0$ et $R$ effectif, de support ne contenant pas $E$.

Si l'on veut des orbifoldes entières (resp. et des morphismes divisibles), on doit remplacer $\left\{m(D) / t_{E, D}\right\}$ par $\left\{\left\lceil m(D) / t_{E, D}\right\rceil\right\}$ (resp. par : $\operatorname{ppcm}_{D}\left\{m(D) / \operatorname{pgcd}\left(t_{E, D}, m(D)\right\}\right.$. 
On impose la condition $m_{Y}(E) \geq 1, \forall E \in W(Y)$, puisqu'elle n'est pas toujours satisfaite (par exemple si $f: Y \rightarrow X$ est un revêtement ramifié de courbes projectives, si $\Delta_{X}=0$, et si $E$ est un point de ramification de $f$ ).

On notera que si $g: Z \rightarrow Y$ est une seconde application holomorphe propre et surjective, alors $(f \circ g)^{+}\left(\Delta_{X}\right) \leq g^{+}\left(f^{+}\left(\Delta_{X}\right)\right)$, mais que l'on n'a, en général, pas égalité.

Par exemple : pour $f$, éclater la surface $X$ en un point lisse du support (non vide, de dimension 1) de $\left(\Delta_{X}\right)$, puis pour $g$, éclater l'intersection de la transformée stricte du support de $\Delta_{X}$ avec le diviseur exceptionnel. Si la composante de $\Delta_{X}$ contenant le premier point éclaté est de multiplicité $m \geq 2$, la multiplicité du diviseur exceptionnel du second éclatement dans $(f \circ g)^{+}\left(\Delta_{X}\right)\left(\right.$ resp. dans $\left.g^{+}\left(f^{+}\left(\Delta_{X}\right)\right)\right)$ est $m / 2$ (resp. $\left.m\right)$.

Definition 2.6 Soit $\mathbb{D}$ le disque unité de $\mathbb{C}$, et $X$ lisse. On note $H o l\left(\mathbb{D},\left(X \mid \Delta_{X}\right)\right)$ l'ensemble, muni de la topologie de la convergence compacte, des applications holomorphes $h: \mathbb{D} \rightarrow X$ qui sont des morphismes orbidolde lorsque $\mathbb{D}($ resp. $X)$ est muni du diviseur orbifolde vide (resp. $\left.\Delta_{X}\right)$. (voir $[C-W 05])$.

Proposition 2.7 Soit $\left(Y \mid \Delta_{Y}\right),\left(X \mid \Delta_{X}\right)$ des orbifoldes géométriques, avec $Y, X$ lisses, et $f: Y \rightarrow X$ holomorphe. Alors :

L'application de composition : $f_{*}: \operatorname{Hol}\left(\mathbb{D},\left(Y \mid \Delta_{Y}\right)\right) \rightarrow \operatorname{Hol}(\mathbb{D}, X)$ définie par $: f_{*}(h):=f \circ h$ a son image contenue dans $\operatorname{Hol}\left(\mathbb{D},\left(X \mid \Delta_{X}\right)\right)$ si $f$ : $\left(Y \mid \Delta_{Y}\right) \rightarrow\left(X \mid \Delta_{X}\right)$ est un morphisme (d'orbifoldes géométriques). Si $\left(Y \mid \Delta_{Y}\right)$ est entière, la réciproque est vraie (ie : $f$ est un morphisme orbifolde si $f_{*}$ a son image contenue dans $\left.\operatorname{Hol}\left(\mathbb{D},\left(X \mid \Delta_{X}\right)\right)\right)$.

Démonstration : Si $f$ est un morphisme d'orbifoldes, la remarque 2.2(2) précédente montre que l'image de $f_{*}$ est contenue dans $\operatorname{Hol}\left(\mathbb{D},\left(X \mid \Delta_{X}\right)\right)$. Réciproquement, supposons que, avec les notations de 2.3, $f^{*}(D)=t . E+R$, avec $t>0$. Supposons que $t . m_{Y}(E)<m_{X}(D)$. Soit $y \in E$ un point générique lisse, et $h: \mathbb{D} \rightarrow Y$ holomorphe telle que $h(0)=y$, et que $h^{*}(E)=m_{Y}(E) \cdot\{0\}$, avec $:\left(h(\mathbb{D}) \cap\left\lceil\Delta_{Y}\right\rceil\right)=\{0\}$. (On peut réaliser ces conditions en restreignant suffisamment $\mathbb{D}$, puisque $\left(Y \mid \Delta_{Y}\right)$ est entière). Alors : $(f \circ h)^{*}(D)=t . h^{*}(E)+h^{*}(R)=t . m_{Y}(E) .\{0\}$. Puisque $t . m_{Y}(E)<m_{X}(D)$, $f$ n'est donc pas un morphisme d'orbifoldes. 


\subsection{Résolution d'une orbifolde géométrique.}

On peut définir comme suit la notion de modèle lisse d'une orbifolde géométrique arbitraire $(X \mid \Delta)$, définie sur un espace complexe normal $X$ algébriquement $\mathbb{Q}$-factoriel, c'est-à-dire tel que tout diviseur de Weil sur $X$ soit $\mathbb{Q}$-Cartier. On note $\operatorname{Sing}(X)$ le lieu singulier de $X$.

Soit $\Delta=\sum_{j}\left(1-1 / m_{j}\right) . D_{j}$ un diviseur orbifolde de $X$ de support $D$, où $m_{j} \in \mathbb{Q} \cap \infty, \forall j$.

Definition 2.8 Une résolution $p:\left(Y \mid \Delta_{Y}\right) \rightarrow(X \mid \Delta)$ de l'orbifolde géométrique $(X \mid \Delta)$ est alors une désingularisation $p: Y \rightarrow X$ de $X$ telle que $p^{-1}((D) \cup \operatorname{Sing}(X))$ soit un diviseur à croisements normaux sur $Y$, et $\left(Y \mid \Delta_{Y}\right)$ une orbifolde géométrique lisse de $Y$ de support contenu dans $p^{-1}((D) \cup \operatorname{Sing}(X))$, telle que $p_{*}\left(\Delta_{Y}\right)=\Delta$, et telle que $p:\left(Y \mid \Delta_{Y}\right) \rightarrow(X \mid \Delta)$ soit un morphisme orbifolde.

Remarque 2.9 Si $p:\left(Y \mid \Delta_{Y}\right) \rightarrow(X \mid \Delta)$ et $p:\left(Z \mid \Delta_{Z}\right) \rightarrow(X \mid \Delta)$ sont deux telles résolutions, et si $u: V \rightarrow Y$ et $v: V \rightarrow Z$ sont biméromorphes (propres), il existe une plus petite orbifolde géométrique $\Delta_{V}$ sur $V$ telle que $u:\left(V \mid \Delta_{V}\right) \rightarrow\left(Y \mid \Delta_{Y}\right)$ et $v:\left(V \mid \Delta_{V}\right) \rightarrow\left(Z \mid \Delta_{Z}\right)$ soient des morphismes orbifoldes biméromorphes élémentaires, de sorte que $p \circ u:\left(V \mid \Delta_{V}\right) \rightarrow(X \mid \Delta)$ et $p \circ v:\left(V \mid \Delta_{V}\right) \rightarrow(X \mid \Delta)$ sont aussi des résolutions, pourvu que les images réciproques de $(D \cup \operatorname{Sing}(X))$ par $p \circ u$ et $p \circ v$ soient des diviseurs à croisements normaux.

- Lorsque $X$ n'est pas $\mathbb{Q}$-factorielle, la notion de résolution d'une orbifolde géométrique n'est pas définie ici (et il ne semble pas immédiat d'en donner une définition naturelle). La situation est donc, ici encore, analogue à celle des résolutions logarithmiques du programme des (Log) modèles minimaux.

\subsection{Faisceaux de formes différentielles sur les orbi- foldes géométriques lisses.}

Notations : Soit $(X \mid \Delta)$ une orbifolde géométrique lisse (ie : $X$ est lisse, et $\lceil\Delta\rceil$ est à croisements normaux). On note $\Delta=\sum_{h \in H}\left(1-1 / m_{h}\right) \cdot D_{h}=$ $\sum_{h \in H} a_{h} \cdot D_{h},[a]=\lfloor a\rfloor$ étant la partie entière du réel $a$, et $\lceil a\rceil:=-\lfloor(-a)\rfloor$ son "arrondi supérieur". Ici les multiplicités $m_{j}$ sont donc des rationnels dans $[1,+\infty[$ ou $+\infty$. 
Soit $q \geq 0$ un entier, et $\Omega_{X}^{q}(\log \lceil\Delta\rceil)$ le faiceau des germes de formes différentielles à pôles logarithmiques dans $\lceil\Delta\rceil$ associé.

- Nous allons définir, pour une orbifolde géométrique lisse $(X \mid \Delta)$ les faisceaux $S^{N}\left(\Omega^{q}(X \mid \Delta)\right)$, analogues des faisceaux $\operatorname{Sym}^{N}\left(\Omega_{X}^{q}\right)$ lorsque $\Delta=0$.

Localement, dans des coordonnées locales $x=\left(x_{1}, \ldots, x_{p}\right)$ adaptées à $\Delta$, c'est-à-dire telles que $\Delta$ ait pour équation ${ }^{5}: \Pi_{j=1}^{j=p} x_{j}^{\left(1-1 / m_{j}\right)}$, ce faisceau admet donc comme $\mathcal{O}_{X}$-module les générateurs : $d x_{J} / x_{J}$, pour $J$ partie ordonnée à $q$ éléments de $\{1, \ldots, p\}$. On a noté $: d x_{J} / x_{J}:=\Lambda_{j \in J} d x_{j} / x_{j}$.

On note alors, pour tous les entiers non-négatifs $q, N: S_{N, q}(X \mid \Delta):=$ $S^{N}\left(\Omega^{q}(X \mid \Delta)\right)$ le sous-faisceau analytique cohérent localement libre ${ }^{6}$ de $\operatorname{Sym}^{N}\left(\Omega_{X}^{q}(\log \lceil\Delta\rceil)\right)$ engendré par les éléments :

$\left\{d x_{(J)} / x_{(J)}:=x^{\lceil k / m\rceil} \cdot \otimes_{l=1}^{l=N} d x_{J_{l}} / x_{J_{l}}=x^{\lceil-k \cdot a\rceil} \cdot \otimes_{l=1}^{l=N} d x_{J_{l}},(J)=\left(J_{1}, \ldots, J_{N}\right)\right\}$,

définis comme suit :

1. Les $J_{l}$ sont des parties ordonnées (croissantes) à $q$ éléments de $\{1, \ldots, p\}$. Les $N$-uplets $\left(J_{1}, \ldots, J_{N}\right)$ considérés sont croissants au sens large pour l'ordre lexicographique sur les parties ordonnées à $q$ éléments de $\{1, \ldots, p\}$.

2. Pour tout $j=1, \ldots, p$, on note $k_{j}$ le nombre d'occurrences de $j$ dans la suite $J_{1}, \ldots, J_{N}$. (C'est-à-dire que $k_{j}=\sum_{l=1}^{l=N} k_{j, l}$, où $k_{j, l}=1$ si $j \in J_{l}$, et $k_{j, l}=0$ sinon).

3. On a aussi noté $k / m$ le $p$-uplet $\left(k_{1} / m_{1}, \ldots, k_{p} / m_{p}\right)$, et $\lceil k / m\rceil$ le $p$-uplet : $\left(\left\lceil k_{1} / m_{1}\right\rceil, \ldots,\left\lceil k_{p} / m_{p}\right\rceil\right)$. Enfin : $x^{\lceil k / m\rceil}:=\prod_{j=1}^{j=p} x_{j}^{\left\lceil k_{j} / m_{j}\right\rceil}$.

4. On a enfin noté $(-k . a)$ le $p$-uplet $\left(k_{1} \cdot a_{1}, \ldots,-k_{p} . a_{p}\right)$, et $\lceil-k . a\rceil$ le $p$ uplet : $\left(\left\lceil-k_{1} \cdot a_{1}\right\rceil, \ldots,\left\lceil-k_{p} \cdot a_{p}\right\rceil\right)$. Enfin $: x^{\lceil-k \cdot a\rceil}:=\Pi_{j=1}^{j=p} x_{j}^{\left\lceil-k_{j} \cdot a_{j}\right\rceil}$, avec $: a_{j}:=$ $\left(1-1 / m_{j}\right)$.

- Cette définition est clairement indépendante des cartes adaptées locales utilisées.

On vérifie alors aisément que l'application naturelle $S_{N, q}(X \mid \Delta) \otimes$ $S_{M}, q(X \mid \Delta) \rightarrow S_{N+M}, q(X \mid \Delta)$ est bien définie (ie : prend bien ses valeurs dans le membre de droite).

Remarque 2.10 1. A nouveau, la définition précédente s'applique avec changement mineur au cas où les multiplicités de $\Delta$ sont réelles.

\footnotetext{
${ }^{5}$ symbolique : les $m_{j}$ sont les $\Delta$-multiplicités des hyperplans de coordonnées.

${ }^{6}$ C'est la propriété cruciale, utilisée constamment dans la suite. Elle permet de négliger les sous-ensembles de codimension 2 ou plus, et donc de ne faire intervenir que le lieu de codimension 1 du diviseur orbifolde.
} 
2. L'origine des faisceaux $S_{N, q}(X \mid \Delta)=S_{N, q}$ (définis dans [Ca01]) est la suivante, pour les orbifoldes géométriques entières : localement, dans la carte $x$ précédente, $X \mid \Delta$ a un revêtement universel local $f: Y \rightarrow X$ donné dans la carte $y=\left(y_{1}, \ldots, y_{p}\right)$ par $: f(y)=x=\left(x_{1}=: y_{1}^{m_{1}}, \ldots, x_{p}=: y_{p}^{m_{p}}\right)^{7}$. Lorsque $N$ est suffisamment divisible, $f^{*}\left(S_{N, q}\right)=\operatorname{Sym}^{N}\left(\Omega_{Y}^{q}\right)$. Pour $N$ général, $S_{N, q}$ est le plus grand sous-faisceau $\mathcal{F}$ de $\operatorname{Sym}^{N}\left(\Omega_{X}(\log (\lceil\Delta\rceil))\right.$ tel que $f^{*}(\mathcal{F}) \subset$ $\operatorname{Sym}^{N}\left(\Omega_{Y}^{q}\right)$.

3. Ces faisceaux ont été utilisés de manière cruciale dans le cadre de la théorie de Nevanlinna dans [C-P05], avec $p=2, q=1$.

4. En général, $S_{N, q}(X \mid \Delta)$ contient, mais n'est pas égal à $\operatorname{Sym}^{N}\left(\Omega^{q}(X \mid \Delta)\right)$.

Exemple 2.11 1. Si $q=\operatorname{dim}(X), S_{N, q}(X \mid \Delta)=N \cdot K_{X}+\sum_{h \in H}\{\lfloor(N(1-$ $\left.\left.\left.1 / m_{h}\right)\right\rfloor . D_{h}\right\}:=N . K_{X}+\lfloor N . \Delta\rfloor:=\left\lfloor N .\left(K_{X}+\Delta\right)\right\rfloor$. Le $\mathbb{Q}$-diviseur $K_{X}+\Delta$ est le fibré canonique de $(X \mid \Delta)$.

2. Si $\Delta_{X}=0, S_{N, q}(X \mid \Delta)=\operatorname{Sym}^{N}\left(\Omega_{X}^{q}\right), \forall N, q$.

3. Si $\Delta=\lceil\Delta\rceil$, alors : $S_{N, q}(X \mid \Delta)=\operatorname{Sym}^{N}\left(\Omega_{X}^{q}(\log (\Delta))\right), \forall N$, q. On a bien sûr : $S_{N, q}(X \mid \Delta) \subset S_{N, q}\left(X \mid \Delta^{\prime}\right)$ si $\Delta \leq \Delta^{\prime}$.

Les faisceaux $S_{N, q}(X \mid \Delta)$ interpolent donc, en général, entre $\operatorname{Sym}^{N}\left(\Omega_{X}^{q}\right)$ et $\operatorname{Sym}^{N}\left(\Omega_{X}^{q}(\log (\lceil\Delta\rceil))\right)$.

4. Soit $\left(\mathbb{P}^{1} / D\right)$ l'orbifolde géométrique logarithmique de dimension 1 , avec $D$ réduit de support 2 points distincts de $\mathbb{P}^{1}$ (par exemple 0 et $\infty$ ). Alors les faisceaux $S_{N, 1}\left(\mathbb{P}^{1} / D\right)$ sont triviaux, de rang 1 . On en déduit que les faisceaux $S_{N, q}\left(\left(\mathbb{P}^{1} / D\right)^{r}\right)$ sont tous triviaux.

Proposition 2.12 Soient $\left(Y \mid \Delta_{Y}\right)$ et $\left(X \mid \Delta_{X}\right)$ des orbifoldes géométriques lisses, et $f: Y \rightarrow X$ holomorphe.

1. Si $f$ induit un morphisme d'orbifoldes géométriques, alors $\left.f^{*}\left(S_{N, q}\left(X \mid \Delta_{X}\right)\right) \subset S_{N, q}\left(Y \mid \Delta_{Y}\right)\right)$ pour tous $N, q$.

2. Si $\left.f^{*}\left(S_{N, 1}\left(X \mid \Delta_{X}\right)\right) \subset S_{N, 1}\left(Y \mid \Delta_{Y}\right)\right)$ pour $N=\operatorname{ppcm}_{j}\left(\operatorname{num}\left(m_{j}\right)\right)$, avec $\Delta_{X}=\sum_{j \in J}\left(1-1 / m_{j}\right)$. $D_{j}$, alors $f$ induit un morphisme d'orbifoldes géométriques. On a noté num $\left(m_{j}\right)$ le numérateur $u_{j}$ de $m_{j}=\frac{u_{j}}{v_{j}}$, si $u_{j}, v_{j} \in \mathbb{Z}$ sont premiers entre eux.

Démonstration : Les notations sont celles introduites ci-dessus.

\footnotetext{
${ }^{7}$ Avec la convention : $x_{i}=y_{i}^{+\infty}:=\exp \left(y_{i}\right)$, ou encore $: d x_{i} / x_{i}=d y_{i}$, si $m_{i}=+\infty$.
} 
Pour 1, il suffit de montrer que, pour tous $N, q, f^{*}\left(x^{\lceil k / m\rceil} \cdot \otimes_{l=1}^{l=N} d x_{J_{l}} / x_{J_{l}}\right) \in$ $S_{N, q}\left(Y \mid \Delta_{Y}\right)$. Puisque ce dernier faisceau est localement libre, il suffit (Hartogs) de vérifier cettte inclusion en codimension un dans $Y$. Soit donc $E \in W(Y)$ et $b \in E$ un point générique. Soit $y=\left(y_{1}, \ldots, y_{n}\right)$ des coordonnées locales de $Y$ en $b$ telles que $E$ ait pour équation locale $y_{1}=0$. Au voisinage de $b$, on a donc : $f(y)=\left(y_{1}^{t_{1}} \cdot f_{1}(y), y_{1}^{t_{2}} \cdot f_{2}(y), \ldots, y_{1}^{t_{p}} \cdot f_{p}(y)\right)$, avec $f_{j}(b) \neq 0$ pour $j \geq 1$. Notre hypothèse est que $t_{j} \cdot m^{\prime} \geq m_{j}$, pour $j=1, \ldots, p$, si $m^{\prime}:=m_{\Delta_{Y}}(E)$.

Donc : $f^{*}\left(d x_{J} / x_{J}\right)=d y_{1} / y_{1} \wedge u_{J}, \forall J$, avec $u_{J}, \forall J$, une $(q-1)$-forme holomorphe, si $|J|=q$, et si $t_{j} \geq 1$ pour un $j \in J$ au moins. Par suite, à des termes holomorphes près : $f^{*}\left(x^{\lceil k / m\rceil} \cdot \otimes_{l=1}^{l=N} d x_{J_{l}} / x_{J_{l}}\right)=g(y) \cdot y_{1}^{s} \cdot\left(\otimes_{l=1}^{l=k^{\prime}}\left(d y_{1} / y_{1} \wedge\right.\right.$ $\left.\left.u_{J_{l}}\right)\right) \otimes_{l=k^{\prime}+1}^{l=N} w_{l}$, avec $g$ et $w_{l}$ holomorphes, et $s:=\sum_{j=1, \ldots, p} t_{j}\left\lceil k_{j} / m_{j}\right\rceil$.

On veut montrer que $s \geq\left\lceil k^{\prime} / m^{\prime}\right\rceil$, où $k^{\prime}$ est l'entier ci-dessus (nombre d'occurences de $d y_{1} / y_{1}$ dans la forme précédente). On a évidemment : $k^{\prime} \leq \sum_{j=1}^{j=p} k_{j}$. Par ailleurs : $t_{j} \cdot m^{\prime} \geq m_{j}, \forall j$, donc : $t_{j} / m_{j} \geq 1 / m^{\prime}, \forall j$. Donc, pour tout $j: t_{j} \cdot\left\lceil k_{j} / m_{j}\right\rceil \geq t_{j} . k_{j} / m_{j} \geq k_{j} / m^{\prime}$. Donc $: s=\sum t_{j} \cdot\left\lceil k_{j} / m_{j}\right\rceil \geq$ $\sum k_{j} / m^{\prime} \geq k^{\prime} / m^{\prime}$. Puisque $s$ est entier, on a donc bien $: s \geq\left\lceil k^{\prime} / m^{\prime}\right\rceil$.

Pour 2, soit $E, D, b, t, f$ comme ci-dessus. On a donc, en particulier : $x_{1}(f(y))=y_{1}^{t_{1}}$. Donc : $f^{*}\left(x_{1}^{\left\lceil N / m_{1}\right\rceil} \cdot\left(d x_{1} / x_{1}\right)^{\otimes N}\right)=t_{1}^{N} \cdot y_{1}^{t_{1}\left\lceil N / m_{1}\right\rceil} \cdot\left(d y_{1} / y_{1}\right)^{\otimes N}$. On a, par hypothèse : $t_{1} \cdot\left\lceil N / m_{1}\right\rceil \geq N / m^{\prime}$. Or, num $\left(m_{1}\right)$ divise $N=$ $\operatorname{ppcm}\left(\operatorname{num}\left(m_{j}\right)\right)$, et on a donc : $t_{1} \cdot N / m_{1}=t_{1} \cdot\left\lceil N / m_{1}\right\rceil \geq N / m^{\prime}$. C'est précisément (multipliée par $N$ ) l'inégalité définissant les morphismes orbifolde.

De l'exemple 2.11 on déduit :

Corollaire 2.13 Si $f:\left(Y \mid \Delta_{Y}\right) \rightarrow\left(X \mid \Delta_{X}\right)$ est un morphisme orbifolde surjectif et génériquement fini entre orbifoldes géométriques lisses, on a : $\left(K_{Y}+\Delta_{Y}\right) \geq f^{*}\left(K_{X}+\Delta_{X}\right)$ (signifiant que la différence est $\mathbb{Q}$-effective). En particulier, $\kappa\left(Y \mid \Delta_{Y}\right) \geq \kappa\left(X \mid \Delta_{X}\right)$ si $Y$ est compacte et connexe.

Remarque 2.14 Dans [Ca04, définition 2.40, p. 541], la notion de morphisme était définie seulement à l'aide des fibrés canoniques. Cette notion semble trop faible pour étudier la catégorie des orbifoldes géométriques dans un cadre biméromorphe.

\subsection{Tenseurs holomorphes orbifoldes.}


On reprend les hypothèses et notations de la section précédente définissant les faisceaux $S_{N, q}(X \mid \Delta)$ sur $(X \mid \Delta)$ lisse. On va définir plus généralement, de manière entièrement similaire, les faisceaux $T_{s}^{r}(X \mid \Delta)$ de tenseurs holomorphes $r$-contravariants et $s$-covariants, muni des opérations de contraction et de tensorisation usuelles. Cette définition résulte d'une discussion avec M. Paun.

Dans des cordonnées locales adaptées $\left(x_{1}, \ldots, x_{n}\right)$, c'est le faisceau $T_{s}^{r}(X \mid \Delta)$ localement libre engendré comme $\mathcal{O}_{X}$-module par les :

$$
t_{v}^{u}:=x^{\lceil(h-k) \cdot a\rceil} \cdot \bigotimes_{j=1}^{j=s} d x_{v(j)} \bigotimes_{i=1}^{i=r} \partial / \partial x_{u(i)},
$$

où $u:[1, r] \rightarrow[1, p]$ et $v:[1, s] \rightarrow[1, p]$ sont des applications quelconques, le $p$-uplet $\lceil(h-k) . a\rceil$ (à valeurs dans $\mathbb{Z}$ ) étant défini comme dans la section précédente.

De la définition on déduit les deux propriété usuelles suivantes :

- On a une application de tensorisation naturelle :

$$
\otimes: T_{s}^{r}(X \mid \Delta) \bigotimes T_{q}^{p}(X \mid \Delta) \rightarrow T_{s+q}^{r+p}(X \mid \Delta), \forall p, q, r, s .
$$

- On a une application de contraction naturelle :

$$
c: T_{s+p}^{r+p}(X \mid \Delta) \rightarrow T_{s}^{r}(X \mid \Delta), \forall p, r, s .
$$

Remarque 2.15 On pourrait définir de même des espaces de jets sur $(X \mid \Delta)$ lisse, jets qui interviennent dans l'étude de l'hyperbolicité.

\subsection{Dimension canonique d'un faisceau différentiel de rang 1}

Notations Soit $(X \mid \Delta)$ une orbifolde géométrique lisse, avec $X$ lisse, compacte et connexe, et $N, q \geq 0$, entiers. On notera $N_{0}:=N_{0}(\Delta)$ le plus petit commun multiple des num $\left(m_{\Delta}(D)\right)$ pour les $D \in W(X)$ tels que $m_{\Delta}(D)$ soient finis.

Soit $L_{N} \subset S_{N, q}(X \mid \Delta)$ un sous faisceau analytique cohérent de rang 1. On notera $\overline{L_{N}} \subset S_{N, q}(X \mid \Delta)$ sa saturation dans $S_{N, q}(X \mid \Delta)$, saturation qui est par définition le plus grand sous-faisceau analytique cohérent de rang un de $S_{N, q}(X \mid \Delta)$ contenant $L_{N}$. Ce faisceau est donc sans cotorsion. 
Soit maintenant $L \subset \Omega_{X}^{q}$ un sous-faisceau analytique cohérent de rang 1, et pour tout $N \geq 0$, soit $L_{N} \subset S_{N, q}(X \mid \Delta)$ l'image de $L^{\otimes N}$ dans $\operatorname{Sym}^{N}\left(\Omega_{X}^{q}\right) \subset S_{N, q}(X \mid \Delta)$, et soit enfin $\overline{L_{N}} \subset S_{N, q}(X \mid \Delta)$ sa saturation dans ce même faisceau.

On a des applications naturelles $\overline{L_{N}} \otimes \overline{L_{N^{\prime}}} \rightarrow \overline{L_{N+N^{\prime}}}$, qui induisent au niveau des sections une structure d'anneau gradué sur $R(X \mid \Delta, L):=$ $\oplus_{N \geq 0} H^{0}\left(X, \overline{L_{N}}\right)$. On notera $R_{0}((X \mid \Delta), L):=\oplus_{M \geq 0} H^{0}\left(X, \overline{L_{M . N_{0}}}\right)$ : c'est évidemment un sous-anneau gradué de $R((X \mid \Delta), L)$.

On notera $\kappa(X \mid \Delta, L)$ le degré de transcendance sur $\mathbb{C}$ de cet anneau, diminué d'une unité si ce degré est au moins 1 ; si ce degré de transcendance est 0 , on notera $\kappa(X \mid \Delta, L)=-\infty$. On définit de même $\kappa_{0}((X \mid \Delta), L)$ à l'aide de l'anneau $R_{0}((X \mid \Delta), L)$.

Lemme 2.16 Si $(X \mid \Delta)$ est lisse, et si $N_{0}=N_{0}(\Delta)$ :

1. Pour tout $N \geq 0$, on a, en codimension un ${ }^{8}$ sur $X:\left(\overline{L_{N}}\right)^{\otimes N_{0}} \subset$ $\left(\overline{L_{N_{0}}}\right)^{\otimes N}=\overline{L_{N . N_{0}}}$.

2. De plus, $H^{0}\left(X,\left(\overline{L_{N_{0}}}\right)^{\otimes N}\right)=H^{0}\left(X, \overline{L_{N . N_{0}}}\right)$

3. $\kappa_{0}((X \mid \Delta), L)=\kappa((X \mid \Delta), L)$.

4. $\kappa((X \mid \Delta), L)=\overline{\lim }_{N \rightarrow+\infty}\left(\log \left(p_{N}(X \mid \Delta, L) / \log N\right)=\max _{N>0}\left(\operatorname{dim}\left(\Phi_{N}(X)\right)\right)\right.$. Ici, $p_{N}(X \mid \Delta, L):=h^{0}\left(X, \overline{L_{N}}\right)$, et $\Phi_{N}(X)$ est l'application méromorphe induite par le système linéaire complet $\overline{L_{N}}$.

En particulier, $\kappa(X \mid \Delta, L) \in\{-\infty, 0,1, \ldots, p:=\operatorname{dim}(X)\}$ est appelé (suivant la terminologie de Moishezon) la dimension canonique de $L$.

La $\mathbb{C}$-algèbre $K(X \mid \Delta, L):=\oplus_{N \geq 0} H^{0}\left(X,\left(\overline{L_{N}}\right)\right.$ est appelée l'algèbre canonique de $L$. Lorsque $L=K_{X}$, on note simplement $K(X \mid \Delta)$ cette algèbre, appelée l'algèbre canonique de $(X \mid \Delta)$.

Démonstration : 1 . Soit $a \in D_{j}$, générique, et $x=\left(x_{1}, \ldots, x_{p}\right), p=$ $\operatorname{dim}(X)$ des coordonnées locales de $X$ en $a$ telles que l'équation de $D_{j}$ en $a$ soit : $x_{1}^{(1-1 / m)}=0$. Soit $w=\left(d x_{1} \wedge u\right)+v$ un générateur local de $L \subset \Omega_{X}^{q}$ en $a$, avec $u, v$ des $(q-1)$-formes holomorphes telles que : $u(a) \neq 0$, et $i_{\left(\partial / \partial x_{1}\right)} v:=$ $v\left(\partial / \partial x_{1}, \ldots\right)=0$. Donc $\overline{L_{N}}$ est engendré près de $a \operatorname{par}\left(x_{1}^{-\lfloor N / m\rfloor}\right) \cdot w^{\otimes N}$ si $v=0$, et par $w^{\otimes N}$ si $v \neq 0$, ceci pour tout $N>0$. Les inclusions annoncées sont donc clairement vraies dans les deux cas.

Si $v=0, \overline{L_{N}}$ est engendré près de $a$ par : $\left(x_{1}^{b} / x_{1}^{N}\right) \cdot w^{\otimes N}$, avec $b=\lceil N / m\rceil$. Puisque $N_{0}=n^{\prime} \cdot \operatorname{num}(m)$, avec $n^{\prime}$ entier, $N_{0}\lceil N / m\rceil \geq\left\lceil N_{0} \cdot N / m\right\rceil=$

\footnotetext{
${ }^{8}$ C'est-à-dire : sur le complémentaire d'un fermé de Zariski de codimension au moins 2.
} 
$N\left\lceil N_{0} / m\right\rceil$. La première inclusion de faisceaux résulte de la première inégalité, et l'égalité de faisceaux de la seconde égalité.

2. Résulte de 1. par Hartogs, puisque $S_{N, q}(X \mid \Delta)$ est localement libre, et entraine immédiatement 3.

Les assertions de 4. sont classiques (voir, par exemple [U75]).

Exemple 2.17 1. Si $q=\operatorname{dim}(X), \kappa\left(X \mid \Delta, K_{X}\right)=\kappa\left(X, K_{X}+\Delta\right)=\kappa(X \mid \Delta)$.

2. Soit $(X \mid \Delta):=\left(\mathbb{P}^{1} / D\right)^{r}, r \geq 1$ l'exemple 4 de 2.11. Pour tout $q \geq 1$ et tout sous-faisceau $L$ de rang 1 de $\Omega_{X}^{q}$, on a donc : $\kappa(X \mid \Delta, L) \leq 0$.

\subsection{Invariance biméromorphe de la dimension cano- nique}

Proposition 2.18 Soit $\left(Y \mid \Delta^{\prime}\right)$ et $(X \mid \Delta)$ des orbifoldes géométriques lisses, $X, Y$ compactes et connexes. Soit $f: Y \rightarrow X$ une application biméromorphe. On a équivalence entre les deux conditions suivantes :

1. $f:\left(Y \mid \Delta^{\prime}\right) \rightarrow(X \mid \Delta)$ est un morphisme orbifolde biméromorphe élémentaire. ${ }^{9}$

2.Il existe $N$, divisible par les multiplicités de $\Delta$ et de $\Delta^{\prime}$, tel que $\left.f^{*}\left(S_{N, 1}(X \mid \Delta)\right) \subset S_{N, 1}\left(Y \mid \Delta^{\prime}\right)\right)$, et $\left.f_{*}\left(S_{N, 1}\left(Y \mid \Delta^{\prime}\right)\right) \subset S_{N, 1}(X \mid \Delta)\right)$.

Démonstration : Si la condition 1. est satisfaite, la première (resp. seconde) des propriétés 2. est satisfaite par 2.12 (resp. par le lemme d'Hartogs, et le fait que $S_{N, 1}(X \mid \Delta)$ ) est localement libre).

Si la condition 2. est satisfaite, alors $f$ est un morphisme orbifolde par la première condition 2. et 2.12. De plus, $f_{*}\left(\Delta^{\prime}\right)=\Delta$ par la seconde condition 2 . En effet, cette condition garantit qu'en codimension 1 , les multiplicités de $\Delta$ coincident avec celles de $\Delta^{\prime}$ sur les transformés stricts des composantes du support de $\Delta$

Théorème 2.19 Soit $X, X^{\prime}$ lisses compactes et connexes, et $u:\left(X^{\prime} \mid \Delta^{\prime}\right) \rightarrow$ $(X \mid \Delta)$ un morphisme orbifolde biméromorphe élémentaire entre orbifoldes géométriques lisses. Soit $L \subset \Omega_{X}^{q}$ un sous-faisceau cohérent de rang 1 , et $L^{\prime}:=u^{*}(L) \subset \Omega_{X^{\prime}}^{q}$. On note, pour tout $N \geq 0 u^{*}: \overline{L_{N}} \rightarrow \overline{L_{N}^{\prime}}$ le morphisme naturel de faisceaux. Alors :

1. $u^{*}: H^{0}\left(X \mid \Delta, L_{N}\right) \rightarrow H^{0}\left(X^{\prime} \mid \Delta^{\prime}, L_{N}^{\prime}\right)$ est un isomorphisme.

2. Donc : $p_{N}(X \mid \Delta, L)=p_{N}\left(X^{\prime} \mid \Delta^{\prime}, L^{\prime}\right)$.

2. $\kappa(X \mid \Delta, L)=\kappa\left(X^{\prime} \mid \Delta^{\prime}, L^{\prime}\right)$.

\footnotetext{
${ }^{9}$ Voir remarque 2.5 , (5) ci-dessus pour la définition.
} 
Démonstration : Soit $A \subset X$ le lieu (de codimension 2 au moins) audessus duquel $u$ n'est pas un isomorphisme. Soit $r: H^{0}\left(X, \overline{L_{N}}\right) \rightarrow H^{0}(X-$ $A, \overline{L_{N}}$ ) la restriction. Par Hartogs, c'est un isomorphisme (d'espaces vectoriels complexes). Soit $r^{\prime}: H^{0}\left(X^{\prime}, \overline{L_{N}^{\prime}}\right) \rightarrow H^{0}\left(X-A, \overline{L_{N}}\right)$ la restriction (composée avec $u$ ). Elle est injective. Puisque $r^{\prime} \circ u^{*}=r, u^{*}$ est un isomorphisme.

Exemple 2.20 Le cas des orbifoldes géométriques logarithmiques est simple : si $\left(X^{\prime} / D^{\prime}\right)$ et $(X / D)$ sont des orbifoldes géométriques logarithmiques lisses (ie : si $X, X^{\prime}$ le sont, et $D, D^{\prime}$ réduites à croisements normaux), ces deux orbifoldes géométriques logarithmiques sont biméromorphes si et seulement s'il existe une suite $w_{j}=u_{j}^{\prime} \circ\left(u_{j}^{-1}\right): X_{j-1} \rightarrow X_{j}$ d'équivalences biméromorphes, pour $j=1, \ldots, m$, telle que $\left(u_{j}^{-1}\left(D_{j}\right)\right)=\left(u_{j}^{\prime}\right)^{-1}\left(D_{j-1}\right)$, avec $\left(X_{0} \mid D_{0}\right)=(X \mid D)$, et $\left(X_{m} \mid D_{m}\right)=\left(X^{\prime} \mid D^{\prime}\right)$. La condition "croisements normaux" n'est pas superflue $: X=\mathbb{P}^{2}$, D la réunion de 3 droites concourantes, et $X^{\prime}=\mathbb{P}^{1} \times \mathbb{P}^{1}$, D' la réunion de 3 fibres de la projection sur le premier facteur, le montrent : la dimension canonique n'est pas même préservée.

Par exemple, les orbifoldes géométriques $(X \mid \Delta):=\left(\mathbb{P}^{1} / D_{1}\right)^{r}, r \geq 1$ de l'exemple 4 de 2.11, et $\left(X^{\prime} \mid \Delta^{\prime}\right):=\left(\mathbb{P}^{r} / D_{r}\right)$, où $D_{r}$ est la réunion réduite et à croisements normaux de $(r+1)$ hyperplans projectifs (distincts) sont biméromorphiquement équivalentes. On en déduit que les $S_{N, q}\left(X^{\prime} \mid \Delta^{\prime}\right)$ sont aussi tous triviaux, et $\kappa\left(X^{\prime} \mid \Delta^{\prime}, L\right) \leq 0$ pour tout sous-faisceau $L$ de rang 1 de $\Omega_{\mathbb{P}^{r}}^{q}$.

Si $U=X-D$ est une variété quasi-projective lisse (connexe), avec $(X \mid D)$ lisse, les propriétés de $(X \mid D)$ qui sont des invariants biméromorphes sont donc des invariants de $U$. Tels sont donc $\kappa(X \mid D), H^{0}\left(X, S^{N}\left(\Omega^{p}(X \mid D)\right)\right.$. Et en particulier $q(U):=H^{0}\left(X, \Omega^{1}(X \mid D)\right.$.

\subsection{Invariance étale de la dimension canonique}

Definition 2.21 Soit $v:\left(Y^{\prime} \mid \Delta^{\prime}\right) \rightarrow(Y \mid \Delta)$ un morphisme orbifolde entre orbifoldes géométriques lisses et entières de même dimension q.

Si $N .\left(K_{Y}+\Delta\right)=v_{*}\left(N .\left(K_{Y^{\prime}}+\Delta^{\prime}\right)\right)$, pour un entier $N$ tel que $N . \Delta$ soit entier, on dit que $v$ est étale en codimension 1.

Proposition 2.22 Soit $v:\left(Y^{\prime} \mid \Delta^{\prime}\right) \rightarrow(Y \mid \Delta)$ un morphisme orbifolde entre orbifoldes géométriques lisses.

1. On a équivalence entre les 3 propriétés (a),(b),(c) suivantes:

(a). $v$ est étale en codimension 1. 
(b). Pour tous $N>0, q \geq 0$, $N$ étant suffisamment divisible, $v^{*}$ : $S_{N, q}(Y \mid \Delta) \rightarrow S_{N, q}\left(Y^{\prime} \mid \Delta^{\prime}\right)$ est un isomorphisme au-dessus du complémentaire d'un fermé analytique $A \subset Y$ de codimension au moins 2.

(c). Il existe un fermé analytique $A \subset Y$ de codimension au moins 2 tel que, au-dessus du complémentaire de $A$ dans $Y, \Delta^{\prime}$ divise exactement $v^{*}(\Delta)$ (ie : pour tout $D^{\prime} \in W(Y)$ rencontrant $\left.\left(Y^{\prime}-v^{-1}(A)\right)\right), r\left(D^{\prime}\right):=$ $m_{\Delta^{\prime}}\left(D^{\prime}\right) / m_{\Delta}\left(v\left(D^{\prime}\right)\right)$ est un entier égal à l'ordre de ramification de $v$ au point générique de $\left.D^{\prime}\right)$.

2. De plus, si $v$ est étale en codimension 1, alors pour tout sous faisceau $L \subset \Omega_{Y}^{q}$ cohérent de rang $1, \kappa(Y \mid \Delta, L)=\kappa\left(Y^{\prime} \mid \Delta^{\prime}, u^{*}(L)\right)$.

Démonstration : Assertion 1 : localement en $b^{\prime} \in Y^{\prime}-A^{\prime}, A^{\prime}:=v^{-1}(A)$, quitte à augmenter un peu $A$ de sorte que $\Delta \cap(Y-A)$ soit lisse, on a des coordonnées $y^{\prime}=\left(y_{1}^{\prime}, \ldots, y_{n}^{\prime}\right)$, ainsi que des coordonnées locales $y=\left(y_{1}, \ldots, y_{n}\right)$ en $b:=v\left(b^{\prime}\right)$ telles que $v\left(y^{\prime}\right)=\left(y_{1}^{\prime r}, y^{\prime} 2, \ldots, y_{n}^{\prime}\right)$, et telles que les équations de $\Delta$ en $b\left(\right.$ resp. $\Delta^{\prime}$ en $\left.b^{\prime}\right)$ soient $: y_{1}^{1-1 / m}\left(\right.$ resp. $\left.y_{1}^{\prime 1-1 / m^{\prime}}\right)$. Un calcul immédiat montre alors que les proriétés (a) et (c) sont simultanément vérifiées, ceci si et seulement si : $r \cdot m^{\prime}=m$. D'où l'équivalence de (a) et (c). Puisque (a) et le cas particulier $q=n$ de (b), et que (c) implique immédiatement (a), l'assertion 1 est établie.

L'assertion 2 résulte de Hartogs, du fait que les faisceaux $S_{N, q}(Y \mid \Delta)$ sont localement libres, et de la propriété (b) ci-dessus. .

\subsection{Inégalité de Bogomolov (version orbifolde)}

Le théorème suivant est dû à F. Bogomolov ([Bo 78]) lorsque $D$ est vide. Sa démonstration s'étend immédiatement au cas logarithmique, en utilisant le fait, dû̀ à P. Deligne ([De 74]), que les sections de $\left.\Omega_{X}^{p}(\log D)\right)$ sont $d$ fermées ${ }^{10}$.

Théorème 2.23 Soit $X$ une variété Kählérienne compacte et connexe ${ }^{11}$, $D$ un diviseur à croisements normaux sur $X$, et $\left.L \subset \Omega_{X}^{p}(\log D)\right)$ un sousfaisceau cohérent de rang 1 . Alors :

(1) $\kappa(X, L) \leq p$.

\footnotetext{
${ }^{10}$ Cette version logarithmique de [Bo 78] a été observée indépendamment par $\mathrm{S}$. Lu.

${ }^{11} \mathrm{Ou}$, plus généralement, biméromorphe à une telle variété.
} 
(2) Si $\kappa(X, L)=p$, alors il existe une (unique) fibration méromorphe $f: X \rightarrow Y$ telle que les saturations dans $\left.\Omega_{X}^{p}(\log D)\right)$ de $L$ et de $f^{*}\left(K_{Y}\right)$ coincident. (En particulier, $\operatorname{dim}(Y)=p$, et $f$ est la fibration d'Iitaka définie par le système linéaire des sections de $\overline{L_{m}}$, pour $m>0$ assez grand, la saturation de $L^{\otimes m}$ étant prise dans $\left.S_{m, p}(X / D)=\operatorname{Sym}^{m}\left(\Omega_{X}^{p}(\log D)\right)\right)$.

Démonstration : Elle est identique à celle de [Bo 78] (dans le cas projectif), ou de [Ca 04] (dans le cas Kähler), en utilisant [De 74] au lieu de la classique fermeture des formes différentielles holomorphes de la théorie de Hodge.

Corollaire 2.24 Soit $X$ une variété Kählérienne compacte et connexe, et $\Delta$ un diviseur orbifolde sur $X$. Soit $L \subset \Omega_{X}^{p}$ un sous-faisceau cohérent de rang 1. Alors :

(1) $\kappa(X \mid \Delta, L) \leq p$.

(2) Si $\kappa(X \mid \Delta, L)=p$, alors il existe une (unique, à équivalence biméromorphe près) fibration méromorphe $f: X \rightarrow Y$ telle que $L$ et $f^{*}\left(K_{Y}\right)$ coincident au point générique de $X$. (En particulier, $\operatorname{dim}(Y)=p$, et $f$ est la fibration d'Iitaka définie par le système linéaire des sections de $\bar{L}_{m}$, pour $m>0$ assez grand, la saturation de $L^{\otimes m}$ étant prise dans $\left.S_{m, p}(X \mid \Delta)\right)$.

Démonstration : Soit $D$ le support de $\Delta$. On a, bien sûr : $\kappa(X \mid \Delta, L) \leq$ $\kappa(X / D, L) \leq p$. D'où (1), par 2.23 (1) ci-dessus, appliqué à $L$ dans $\left.\Omega_{X}^{p}(\log D)\right)$. Si on a égalité, on a aussi $: p=\kappa(X \mid \Delta, L) \leq \kappa(X / D, L) \leq p$. D'où (2), en appliquant de la même façon 2.23 (2)•

\subsection{Orbifoldes géométriques log-canoniques et klt}

Ces notions sont définies comme dans le cadre du PMML (=LMMP en Anglais), avec la condition additionnelle de $\mathbb{Q}$-factorialité.

\section{Definition 2.25}

- L'orbifolde géométrique $(X \mid \Delta)$ est dite log-canonique si :

1. $X$ est $\mathbb{Q}$-factorielle.

2. Le faisceau $\omega_{X}=K_{X}$ est $\mathbb{Q}$-Cartier.

3.Pour toute (ie : pour une) résolution $p:\left(Y \mid \Delta_{Y}\right) \rightarrow(X \mid \Delta)$, on a: $K_{X}+\Delta_{Y} \geq p^{*}\left(K_{X}+\Delta\right)$.

- L'orbifolde géométrique $(X \mid \Delta)$ est dite klt si, pour une (ie : pour toute) résolution $p:\left(Y \mid \Delta_{Y}\right) \rightarrow(X \mid \Delta)$, on $a:\left(K_{X}+\Delta_{Y}\right)-p^{*}\left(K_{X}+\Delta\right):=$ E est un diviseur effectif supporté sur le diviseur exceptionnel de $p$ tout entier. 
Exemple 2.26 Les orbifoldes géométriques lisses sont log-canoniques, et klt si et seulement si à multiplicités finies.

Remarque 2.27 Soit $p:\left(Y \mid \Delta_{Y}\right) \rightarrow(X \mid \Delta)$ une résolution d'une orbifolde géométrique log-canonique. Alors $p^{*}\left(H^{0}\left(X, N\left(K_{(X \mid \Delta)}\right)=H^{0}\left(Y, N \cdot K_{\left(Y \mid \Delta_{Y}\right)}\right)\right.\right.$ est indépendant de la résolution choisie, pour tous $N>0$.

Question 2.28 Soit $p:\left(Y \mid \Delta_{Y}\right) \rightarrow(X \mid \Delta)$ une résolution d'une orbifolde géométrique log-canonique.

Pour tous $N>0, q>0$, le faisceau $S^{N}\left(\Omega^{q}(X \mid \Delta)\right):=p_{*}\left(S^{N}\left(\Omega^{q}\left(Y \mid \Delta_{Y}\right)\right)\right.$ est-il indépendant de la résolution $p$ ?

Si oui, alors : $p^{*}\left(H^{0}\left(X, S^{N}\left(\Omega^{q}(X \mid \Delta)\right)\right)\right)=H^{0}\left(Y,\left(S^{N}\left(\Omega^{q}\left(Y \mid \Delta_{Y}\right)\right)\right)\right)$ est indépendant de la résolution choisie, pour tous $N, q$.

Question 2.29 Supposons que $(X \mid \Delta)$ soit une orbifolde géométrique log-canonique, et que les faisceaux $\left.S^{N}\left(\Omega^{q}(X \mid \Delta)\right)\right)$ soient biens définis (indépendants de la résolution de $(X \mid \Delta)$ ). Les faisceaux $S^{N}\left(\Omega^{q}(X \mid \Delta)\right)$ ) sontils alors réflexifs?

Pour $q=n:=\operatorname{dim}(X)$, c'est une conséquence de la définition. Lorsque $q=1,(n-1)$, une réponse positive partielle est fournie dans $[G-K-K]$.

\subsection{2 Équivalence biméromorphe.}

Rappelons (définition $2.3(5))$ que $f:\left(Y \mid \Delta_{Y}\right) \rightarrow\left(X \mid \Delta_{X}\right)$ est un morphisme orbifolde biméromorphe élémentaire si c'est un morphisme orbifolde, si $f: Y \rightarrow X$ est birationnel, et si $f_{*}\left(\Delta_{Y}\right)=\Delta_{X}$. On suppose ici que $Y, X$ sont $\mathbb{Q}$-factorielles. Noter que les trois propriétés sont indépendantes (deux d'entre elles n'impliquent pas la troisième).

Definition 2.30 On dira que deux orbifoldes géométriques log-canoniques sont biméromorphiquement équivalentes s'il existe une chaine de morphismes orbifoldes biméromorphes élémentaires les reliant, et tels que chacunes des orbifoldes géométriques de cette chaine soit log-canonique. De manière équivalente : c'est la relation déquivalence engendrée par les morphismes orbifolde biméromorphes élémentaires entre orbifoldes géométriques log-canoniques.

Remarque 2.31 Il résulte de a remarque 2.27 que la dimension canonique d'une orbifolde géométrique log-canonique est un invariant birationnel, au sens défini ci-dessus. 
L'équivalence biméromorphe dans cette catégorie présente des différences notables avec le cas non orbifolde géométrique. En particulier : l'exemple 2.32 ci-dessous montre que deux orbifoldes géométriques lisses (projectives) biméromorphiquement équivalentes ne sont pas nécessairement dominées par une troisième qui leur est biméromorphiquement équivalente. Cette différence complique considérablement l'étude de l'équivalence biméromorphe orbifolde.

Exemple 2.32 (transformation de Cremona) Soit $X:=\mathbb{P}^{2}$, et 3 points $a, b, c \in X$ non alignés. On note $A, B, C$ les trois droites projectives de $X$ passant par 2 de ces 3 points. Soit $u: X^{\prime} \rightarrow X$ l'éclaté de $X$ en les 3 points $a, b, c$, et $A^{\prime}, B^{\prime}, C^{\prime}$ les transformées strictes des droites $A, B, C$ dans $X^{\prime}$.

On note enfin $E, F, G$ les trois diviseurs irréductibles de $u$. Alors $X^{\prime}$ admet une seconde contraction $v: X^{\prime} \rightarrow Y$ sur $Y \cong \mathbb{P}^{2}$, qui contracte $A^{\prime}, B^{\prime}, C^{\prime}$ sur trois points $a^{\prime}, b^{\prime}, c^{\prime} \in Y$, et $E, F, G$ sur trois droites projectives $E^{\prime}, F^{\prime}, G^{\prime}$ de $Y \cong \mathbb{P}^{2}$. ( $v \circ u^{-1}: X \rightarrow Y$ n'est donc autre que la transformation de Cremona).

On peut munir $X^{\prime}$ des deux structures orbifoldes géométriques (logarithmiques):

$\Delta:=(E+F+G)$ et $\Delta^{\prime}:=\left(A^{\prime}+B^{\prime}+C^{\prime}\right)$.

Alors : $u:\left(X^{\prime} \mid \Delta\right) \rightarrow X$ et $v:\left(X^{\prime} \mid \Delta^{\prime}\right) \rightarrow Y$ sont des morphismes orbifoldes élémentaires, puisque le support de $\Delta$ (resp. $\left.\Delta^{\prime}\right)$ est u-exceptionnel (resp. v-exceptionnel).

De plus, $u: X^{\prime}=\left(X^{\prime} \mid 0\right) \rightarrow X$ et $v: X^{\prime} \rightarrow Y$ sont des morphismes orbifoldes élémentaires, par dédinition même.

Donc, l'identité ensembliste $1_{X^{\prime}}:\left(X^{\prime} \mid \Delta\right) \rightarrow X^{\prime}=\left(X^{\prime} \mid 0\right)$ et $1_{X^{\prime}}:\left(X^{\prime} \mid \Delta^{\prime}\right) \rightarrow X^{\prime}$ sont des morphismes orbifoldes birationnels (non élémentaires).

Par suite, $\left(X^{\prime} \mid \Delta\right)$ et $\left(X^{\prime} \mid \Delta^{\prime}\right)$ sont birationnellement équivalentes.

Soit maintenant $\Delta^{\prime \prime}:=\sup \left(\Delta, \Delta^{\prime}\right)=\left(A^{\prime}+B^{\prime}+C^{\prime}+E+F+G\right)$ : c'est un diviseur orbifolde (logarithmique) sur $X^{\prime}$. De plus, $\kappa\left(X^{\prime} \mid \Delta^{\prime \prime}\right)=\kappa(X / D)=0$, si $D:=(A+B+C)$. Donc $\left(X^{\prime} \mid \Delta^{\prime \prime}\right)$ n'est birationnellement équivalent ni à $\left(X^{\prime} \mid \Delta\right)$ ni à $\left(X^{\prime} \mid \Delta^{\prime}\right)$, puisque $\kappa\left(X^{\prime} \mid \Delta\right)=\kappa(X \mid 0)=-\infty$.

Si l'orbifolde géométrique lisse $\left(Z \mid \Delta_{Z}\right)$ domine (par des morphismes orbifoldes) $g:\left(Z \mid \Delta_{Z}\right) \rightarrow\left(X^{\prime} \mid \Delta\right)$ et $g^{\prime}:\left(Z \mid \Delta_{Z}\right) \rightarrow\left(X^{\prime} \mid \Delta^{\prime}\right)$, elle domine aussi $\left(X^{\prime} \mid \Delta^{\prime \prime}\right)$. En effet, on a, ensemblistement $g=g^{\prime}$. Si $E^{\prime} \in W\left(X^{\prime}\right)$, si $H \in W(Z)$, et si $g^{*}\left(E^{\prime}\right)=t . H+\ldots$, avec $t>0$, on a : t. $m_{Z}(F) \geq$ 
$\sup \left\{m_{\Delta}\left(E^{\prime}\right), m_{\Delta^{\prime}}\left(E^{\prime}\right)\right\}$, par hypothèse. Mais c'est justement la conclusion cherchée.

Donc $\kappa\left(Z \mid \Delta_{Z}\right) \geq \kappa\left(X^{\prime} \mid \Delta^{\prime \prime}\right)=0$, et $\left(Z \mid \Delta_{Z}\right)$ n'est pas birationnelle à $\left(X^{\prime} \mid \Delta\right)$.

Exemple 2.33 Le phénomène précédent (deux orbifoldes géométriques birationnellement équivalentes non dominées par une troisième qui leur est birationnellement équivalente) existe aussi pour les orbifoldes géométriques à multiplicités (entières) finies.

Soit $B$ une courbe elliptique, et $b_{i} \in B, i=1,2$ deux points distincts dont la différence $\left(b_{2}-b_{1}\right) \in A u t^{0}(B)$ est un élément d'ordre 2 . Soit $\left.X:=B \times \mathbb{P}^{1}\right)$. Donc $X$ est munie des projection $p: X \rightarrow B$ et $q: X \rightarrow \mathbb{P}^{1}$. Soit $D_{i}, i=1,2$ deux sections disjointes de $q$, et soit $F_{i}:=p^{-1}\left(b_{i}\right), i=1,2$. On note $a_{i} \in X$ l'intersection de $D_{i}$ et de $F_{i}$.

Soit $t: X " \rightarrow X$ l'éclatement de $X$ en les points $a_{i}, i=1,2, F^{\prime \prime}{ }_{i}$ la transformée stricte de $F_{i}$ dans $X "$ ", et $E^{\prime \prime}{ }_{i}, i=1,2$ le diviseur exceptionnel de $t$ au-dessus de $a_{i}$. Soit $t^{\prime}: X^{\prime \prime} \rightarrow X^{\prime}$ la contraction des deux (-1)-courbes $F^{\prime \prime}{ }_{i}, i=1,2$ dans $X^{\prime \prime}, E_{i}^{\prime} \subset X^{\prime}$ l'image de $E^{\prime \prime}{ }_{i}$ dans $X^{\prime}$, et $D_{i}^{\prime}, i=1,2$ $l^{\prime}$ 'image de $D_{i}$ dans $X^{\prime}$ par $t^{\prime}$. Alors il existe une fibration $b^{\prime}: X^{\prime} \rightarrow B$ telle que $p^{\prime} \circ t^{\prime}=p \circ t$. Et $E_{i}^{\prime}=\left(p^{\prime}\right)^{-1}\left(b_{i}\right), i=1,2$. Par le choix de $\left(b_{2}-b_{1}\right)$, il existe un isomorphisme $f: X \rightarrow X^{\prime}$ qui échange $F_{1}$ et $F_{2}$, ainsi que $D_{1}$ et $D_{2}$.

Donc $f$ se relève en une involution $f$ "de $X "$ qui échange $D{ }_{1}$ et $D{ }_{2}$, et envoit F" ${ }_{1}$ sur $E{ }_{2}$, et $E "{ }_{1}$ sur $F{ }_{2}$.

Soit $m>1$ un entier. On munit $X "$ de deux diviseurs orbifolde $\Delta{ }^{\prime}{ }_{i}$, avec $\Delta "_{1}:=(1-1 / m) \cdot\left[D{ }_{1}+F{ }_{1}+E{ }_{2}+D{ }_{2}\right]$, et $\Delta{ }_{2}:=(1-1 / m) \cdot\left[D{ }_{1}+\right.$ $\left.F^{\prime \prime}{ }_{2}+E^{\prime \prime}{ }_{1}+D{ }_{2}\right]$. On a donc :

$\Delta ":=\sup \left\{\Delta{ }_{1}, \Delta{ }_{2}\right\}=(1-1 / m) \cdot\left[D{ }_{1}+E "_{1}+E{ }_{2}+F{ }_{1}+F{ }_{2}+D{ }_{2}\right]$.

On vérifie immédiatemment que $\Delta{ }_{1}$ et $\Delta{ }{ }_{2}$ sont biméromorphiquement équivalentes. En effet, $t:\left(X " \mid \Delta{ }_{1}\right) \rightarrow(X \mid \Delta)$ et $t^{\prime}:\left(X{ }^{\prime \prime} \mid \Delta{ }_{2}\right) \rightarrow\left(X^{\prime} \mid \Delta^{\prime}\right)$ sont des morphismes orbifoldes biméromorphes élémentaires si l'on définit: $\Delta:=(1-1 / m) \cdot\left[D_{1}+D_{2}\right]$ et $\Delta^{\prime}:=(1-1 / m) \cdot\left[D_{1}^{\prime}+D_{2}^{\prime}\right]$. De plus, il existe un isomorphisme $g:(X \mid \Delta) \rightarrow\left(X^{\prime} \mid \Delta^{\prime}\right)$, induit par $f$.

On en déduit que $h^{0}\left(X^{\prime \prime} \mid \Delta^{\prime \prime}{ }_{i}, S^{N}\left(\Omega^{1}\left(X^{\prime \prime} \mid \Delta^{\prime \prime}{ }_{i}\right)\right)\right)=1, \forall N>0$.

Par ailleurs, $t:\left(X " \mid \Delta^{\prime \prime}\right) \rightarrow\left(X \mid \Delta^{+}\right)$est un morphisme orbifolde élémentaire si $\Delta^{+}:=(1-1 / m) \cdot\left[F_{1}+F_{2}+D_{1}+D_{2}\right]$. D'où l'on déduit que : $h^{0}\left(X^{\prime \prime} \mid \Delta^{\prime \prime}, S^{N}\left(\Omega^{1}\left(X^{\prime \prime} \mid \Delta^{\prime \prime}\right)\right)\right) \geq h^{0}\left(B, S^{N}\left(\Omega^{1}\left(B \mid \Delta_{B}\right)\right)\right) \cong 2 N .(1-1 / m)$, 
puisque $p:\left(X^{\prime \prime} \mid \Delta^{\prime \prime}\right) \rightarrow\left(B \mid \Delta_{B}\right)$ est un morphisme orbifolde, si $\Delta_{B}:=$ $(1-1 / m) \cdot\left(b_{1}+b_{2}\right)$.

En particulier, $(X " \mid \Delta ")$ n'est pas biméromorphe à $\left(X " \mid \Delta{ }_{i}\right)$, puisque l'équivalence biméromorphe préserve les sections de $S^{N}\left(\Omega^{q}\right)$.

Definition 2.34 Si $(X \mid \Delta)$ et $\left(X \mid \Delta^{\prime}\right)$ sont deux orbifoldes géométriques lisses, avec $\Delta \geq \Delta^{\prime}$, l'identité de $X v:=1_{X}:(X \mid \Delta) \rightarrow\left(X \mid \Delta^{\prime}\right)$ est un morphisme orbifolde, qui est un accroissement élémentaire s'il existe deux modifications élémentaires $u:(X \mid \Delta) \rightarrow\left(Y \mid \Delta_{Y}\right)$ et $u^{\prime}:\left(X \mid \Delta^{\prime}\right) \rightarrow\left(Y \mid \Delta_{Y}\right)$ telles que $u^{\prime} \circ 1_{X}=u$ (ie : $u=u^{\prime}$ ensemblistement).

Si v" $:=1_{X}:\left(X \mid \Delta^{\prime \prime}\right) \rightarrow\left(X \mid \Delta^{\prime}\right)$ est un second accroissement élémentaire, l'application biméromorphe composée $\left(v^{\prime \prime}\right)^{-1} \circ v=1_{X}:(X \mid \Delta) \rightarrow(X \mid \Delta$ ") sera appelée une symétrie élémentaire.

Exemple 2.35 Dans l'exemple 2.32 ci-dessus, $1_{X^{\prime}}:\left(X^{\prime} \mid \Delta\right) \rightarrow\left(X^{\prime} \mid \Delta^{\prime}\right)$ est une symétrie élémentaire, composée des morphismes orbifoldes (égaux à $1_{X^{\prime}}$ ensemblistement) sur $X^{\prime}=(X \mid 0)$.

Definition 2.36 Une suite d'équivalences biméromorphes orbifoldes $g_{j}$ : $\left(X_{j} \mid \Delta_{j}\right) \rightarrow\left(X_{j-1} \mid \Delta_{j-1}\right)$ est dite ascendante si chacune des $h_{j}$ est ensemblistement une application holomorphe biméromorphe. Les modifications orbifolde élémentaires et les symétries élémentaires sont donc des suites ascendantes (à un seul élément) d'équivalences biméromorphes orbifolde.

Nous énonçons sans démonstration un résultat qui n'est pas utilisé dans la suite, substitut faible dans le cadre orbifolde géométrique au fait que deux variétés biméromorphes sont dominées par une troisième :

Théorème 2.37 Toute équivalence biméromorphe $h:\left(X_{0} \mid \Delta_{0}\right) \rightarrow \rightarrow$ $\left(X_{N} \mid \Delta_{N}\right)$ entre orbifoldes géométriques lisses est une composée $h=f \circ g^{-1}$ de deux équivalences biméromorphes $f:(X \mid \Delta) \rightarrow\left(X_{0} \mid \Delta_{0}\right)$ et $g:(X \mid \Delta) \rightarrow$ $\left(X_{N} \mid \Delta_{N}\right)$, où $f$ (resp. $g$ ) est composée d'une suite ascendante de modifications orbifoldes élémentaires et de symétries élémentaires.

\subsection{Restriction à une sous-variété.}

Definition 2.38 Soit $(Y \mid \Delta)$ une orbifolde géométrique lisse, et $j: V \rightarrow Y$ l'inclusion d'une sous-variété ${ }^{12}$ non contenue dans le support de $\Delta$.

\footnotetext{
${ }^{12}$ ie : un sous-ensemble analytique fermé irréductible.
} 
L'orbifolde géométrique $j^{\prime}:\left(V^{\prime} \mid \Delta^{\prime}\right) \rightarrow(Y \mid \Delta)$ est une restriction de $\Delta$ à $V$ si :

1. $j^{\prime}: V^{\prime} \rightarrow V$ est une résolution de $V$,

2. $\left(j^{\prime}\right)^{-1}(\operatorname{supp}(\Delta))$ est un diviseur de $V^{\prime}$ à croisements normaux.

3. $j^{\prime}:\left(V^{\prime} \mid \Delta^{\prime}\right) \rightarrow(Y \mid \Delta)$ est un morphisme orbifolde tel que, de plus :

4. pour tout diviseur orbifolde $\Delta$ " sur $V^{\prime}$ satisfaisant les conditions $1,2,3$ précédentes, on $a: \Delta " \geq \Delta$.

En particulier, l'orbifolde $\left(V^{\prime} \mid \Delta^{\prime}\right)$ est donc lisse, et pour toute résolution $j^{\prime}: V^{\prime} \rightarrow V$ satisfaisant la condition 2 ci-dessus, il existe un unique diviseur orbifode $\Delta^{\prime}$ sur $V^{\prime}$ satisfaisant les conditions 3,4 .

Proposition 2.39 Soit $(Y \mid \Delta)$ une orbifolde géométrique lisse, et $j: V \rightarrow$ $Y$ l'inclusion d'une sous-variété non contenue dans le support de $\Delta$. Soit $\left(V^{\prime} \mid \Delta_{V^{\prime}}\right)$ une restriction de $\Delta \grave{a} V$.

1. Si $u: V^{\prime \prime} \rightarrow V^{\prime}$ est une modification propre telle que $\left(j^{\prime} \circ u\right)^{-1}(\operatorname{supp}(\Delta))$ est à croisements normaux, et si $\left(V^{\prime \prime} \mid \Delta_{V}\right.$ ") est la restriction de $\Delta \grave{a} V$ de support $V^{\prime \prime}$, alors $u:\left(V^{\prime \prime} \mid \Delta_{V^{\prime \prime}}\right) \rightarrow\left(V^{\prime} \mid \Delta_{V^{\prime}}\right)$ est une modification orbifolde élémentaire (et en particulier un morphisme orbifolde).

2. Deux restrictions minimales de $\Delta$ à $V$ sont biméromorphiquement équivalentes au sens orbifolde géométrique.

\section{Démonstration :}

1. Montrons que $u$ est un morphisme orbifolde. Soit donc $F \in W\left(V^{\prime}\right)$ et $E \in W(Y)$ choisi tel que $\left(j^{\prime}\right)^{*}(E)=t . F+\ldots$, avec $t>0$ et $t . m_{\Delta_{V^{\prime}}}(F)=$ $m_{\Delta}(E)$, l'égalité résultant de la minimalité de $\Delta_{V^{\prime}}$. Si $F^{\prime} \in W\left(V^{\prime \prime}\right)$ est le transformé strict de $F$ dans $V^{\prime \prime}$, on a donc : $\left(j^{\prime} \circ u\right)^{*}(E)=t . F^{\prime}+\ldots$, et par hypothèse, $t . m_{\Delta_{V^{\prime}}}\left(F^{\prime}\right) \geq m_{\Delta}(E)$. Donc $m_{\Delta_{V^{\prime \prime}}}\left(F^{\prime}\right) \geq m_{\Delta_{V^{\prime}}}(F)$, et $u$ est un morphisme orbifolde. En fait, puisque $\Delta_{V}$ " est minimale, on a égalité, puisque $m_{\Delta_{V},}\left(F^{\prime}\right):=\inf \left\{m_{\Delta}(E) / t\right\}$, pour $E, t$ comme ci-dessus. Donc $u_{*}\left(\Delta_{V} "\right)=$ $\Delta_{V^{\prime}}$.

2. Résulte de 1 . et de la définition de l'équivalence biméromorphe orbifolde.

Exemple 2.40 Pour les courbes, on a unicité de la restriction (minimale ou pas). On va traiter ici un cas particulier (local, près de $(0,0) \in X$ ), lorsque $V \subset X$ est une courbe irréductible dans une surface lisse $X=\mathbb{C}^{2}$. 
Supposons que $V$ soit lisse, et ait en $(0,0)$ un ordre de contact t avec $\Delta$, de multiplicité $m$, de support $D$, d'équation $y=0$, si $(x, y)$ sont des coordonnées locales de $X$ en $(0,0)$. C'est le cas si, par exemple, une équation locale de $V$ en $(0,0)$ est $: y^{t}=x^{s}$, avec $t, s$ entiers premiers entre eux. On va calculer la multiplicité $\mu$ dans $g:\left(V^{\prime} \mid \Delta_{V^{\prime}}\right) \rightarrow V$ de $v:=g^{-1}(0,0)$, si $g: V^{\prime} \rightarrow V$ est la normalisation de $V$.

Par définition même, $g^{*}(D)=t .\{v\}+\ldots$ Donc, $\mu=\sup \{1, m / t\}$ (et $\mu=\lceil m / t\rceil$ si l'on se place dans la catégorie des orbifoldes géométriques entières). Si $\operatorname{supp}(\Delta)$ a 2 branches en $(0,0)$, il faut prendre pour $\mu$ le sup des 2 expressions ainsi obtenues.

Remarque 2.41 La restriction ne commute pas avec l'équivalence biméromorphe. Si $(Y \mid \Delta) \sim\left(Y_{1} \mid \Delta_{1}\right)$, et si $V_{1} \subset Y_{1}$ est la transformée stricte de $V$, deux restrictions de $\Delta \grave{a} V$ et de $\Delta_{1} \grave{a} V_{1}$ ne sonrt en général pas biméromorphiquement équivalentes. Considérer par exemple $V$, une droite projective de $Y:=\mathbb{P}^{2}$, avec $\Delta:=0$, et $u: Y_{1} \rightarrow Y$ l'éclatement de $Y$ en un point $a \in V$, si $\Delta_{1}:=(1-1 / m)$.E, avec $m>1$ et $E$ le diviseur exceptionnel de $u$.

On restreindra néammoins des applications méromorphes dans le cadre orbifolde biméromorphe :

Definition 2.42 Soit $f:(Y \mid \Delta) \rightarrow X$ une application méromorphe propre et surjective, et $V$ une sous-variété $V$ de $Y$ qui n'est contenue ni dans le lieu d'indétermination de $f$, ni dans le support de $\Delta$. On suppose l'orbifolde géométrique $(Y \mid \Delta)$ lisse (et $X$ normale). On appellera restriction de $f$ à $V$, et on notera $f_{V}:\left(V \mid \Delta_{V}\right) \rightarrow X$ la composée $f_{V}:=f^{\prime} \circ j^{\prime}:\left(V^{\prime} \mid \Delta_{V^{\prime}}\right) \rightarrow X$, pour $j^{\prime}:\left(V^{\prime} \mid \Delta_{V^{\prime}}\right) \rightarrow\left(Y^{\prime} \mid \Delta^{\prime}\right)$ une restriction de $\Delta \grave{a} V$ comme ci-dessus, choisie telle que, de plus, $f^{\prime}:=f \circ v: V^{\prime} \rightarrow X$ soit holomorphe.

Remarque 2.43 En général, les invariants biméromorphes de $f_{V}$ dépendent du choix de la restriction. Voir néammoins $\$ 7.3$ pour une situation importante dans laquelle on a indépendance de la dimension canonique lors de ces choix, et §3.4 pour une autre situation particulière. 


\section{BASE ET FIBRE ORBIFOLDES D'UN MORPHISME}

\subsection{Base orbifolde d'un morphisme}

Definition 3.1 On appellera morphisme toute application $f: Y \rightarrow X$, holomorphe propre et surjective, entre espaces analytiques complexes $Y, X$ normaux. Une fibration est un morphisme à fibres connexes.

Soit $\Delta_{Y}:=\Delta$ un diviseur orbifolde sur $Y$.

On note $m\left(\Delta_{Y}\right): W(Y) \rightarrow \mathbb{Q} \cup\{\infty\}:=\overline{\mathbb{Q}}$ la multiplicité de $\Delta_{Y}$.

Si $D \in W(X)$, et si $f: Y \rightarrow X$ est un morphisme, on écrit : $f^{*}(D)=$ $\sum_{j \in J(f, D)} m_{j} . D_{j}+R$, où $R$ est le plus grand diviseur de $X$ de support contenu dans $f^{*}(D)$, et $f$-exceptionnel (ie : tel que la codimension de $f_{*}(R)$ dans $Y$ soit au moins 2$)$. Remarquer que les entiers $m_{j}$ sont bien définis, même si $X$ n'est pas $\mathbb{Q}$-factorielle.

Definition 3.2 (Voir $\left[C a 04,1.29\right.$, p. 523]) Soit $\left(Y \mid \Delta_{Y}\right)$ une orbifolde géométrique. La base orbifolde $\left(X \mid \Delta\left(f, \Delta_{Y}\right)\right)$ du morphisme $f:\left(Y \mid \Delta_{Y}\right) \rightarrow$ $X$ est l'orbifolde géométrique $\left(X \mid \Delta\left(f, \Delta_{Y}\right)\right)$ définie par la multiplicité $m\left(f, \Delta_{Y}\right): W(X) \rightarrow \mathbb{Q} \cup\{\infty\}$ telle que, pour tout $D \in W(X):$ $m\left(f, \Delta_{Y} ; D\right):=i n f_{j \in J(f, D)}\left\{m_{j} \cdot m_{\Delta_{Y}}\left(E_{j}\right)\right\}$.

Lorsque $\Delta_{Y}=0, \Delta(f, 0)$ est simplement notée $\Delta(f)$.

La définition précédente est celle des catégories Georb $^{Q}$ et Georb ${ }^{Z}$ (voir définition 2.4).

Dans Georb ${ }^{\text {div }}$, on remplace :

$m\left(f, \Delta_{Y} ; D\right):=i n f_{j \in J(f, D)}\left\{m_{j} \cdot m_{\Delta_{Y}}\left(E_{j}\right)\right\}$ ci-dessus par :

$m\left(f, \Delta_{Y} ; D\right):=\operatorname{pgcd}_{j \in J(f, D)}\left\{m_{j} . m_{\Delta_{Y}}\left(E_{j}\right)\right\}$.

On précisera si nécessaire la catégorie considérée en notant : $\left(X \mid \Delta\left(f, \Delta_{Y}\right)\right)^{*}$, ou $\left.: \Delta\left(f, \Delta_{Y}\right)\right)^{*}$, avec $: *=Z, Q$, div selon le cas.

\section{Remarque 3.3}

1. Même si $X$ et $\left(Y \mid \Delta_{Y}\right)$ sont lisses, $\left(X \mid \Delta\left(f, \Delta_{Y}\right)\right)$ n'est pas lisse, en général.

2. Si $f:(Y \mid \Delta) \rightarrow\left(X \mid \Delta^{\prime}\right)$ est un morphisme orbifolde, on a : $\Delta^{\prime} \leq$ $\Delta(f, \Delta)$. En général, si $X$ est lisse, $f:(Y \mid \Delta) \rightarrow(X \mid \Delta(f, \Delta))$ n'est pas un 
morphisme orbifolde (à cause des diviseurs $f$-exceptionnels de $Y$, négligés dans la définition de $\left.\Delta\left(f, \Delta_{Y}\right)\right)$. Si $f$ est un morphisme fini, c'est cependant le cas (ceci résulte de 3.13, assertion 2, ci-dessous). On peut toujours, en augmentant les multiplicités des diviseurs $f$-exceptionnels, faire de $f:\left(Y \mid \Delta_{Y}^{+}\right) \rightarrow(X \mid \Delta(f, \Delta))$ un morphisme orbifolde sans changer $\Delta(f, \Delta)$.

3. Si $X$ est une courbe (lisse), $f:(Y \mid \Delta)^{*} \rightarrow\left(X \mid \Delta\left(f, \Delta_{Y}\right)^{*}\right)$ est un morphisme dans la catégorie Georb*, avec $*=Z, Q$, div, puisqu'il n'y a alors pas de diviseur $f$-exceptionnel. Dans ce cas, $\left(X \mid \Delta\left(f, \Delta_{Y}\right)^{*}\right.$ est aussi la plus grande structure orbifolde sur $X$ rendant $f$ un morphisme dans la catégorie Georb*.

4. Si $f=g \circ h$, avec $h: Y \rightarrow X^{\prime}$ une fibration, et $g: X^{\prime} \rightarrow X$ finie (donc $g \circ h$ est la factorisation de Stein du morphisme $f$ ), alors : $\Delta(f, \Delta)=$ $\Delta(g, \Delta(h, \Delta))$ peut être calculée en 2 étapes. (Ceci résulte aussi de 3.13 cidessous). Si $g$ n'est pas finie, on a seulement : $\Delta(f, \Delta) \geq \Delta(g, \Delta(h, \Delta))$. Voir ci-dessous pour les cas d'égalité.

5. La définition 3.2 est motivée, entre autres, par la propriété suivante $([\mathrm{Ca} 04,1.30]):$ si $\Delta_{Y}=\Delta(g)$, pour une fibration $g: Z \rightarrow Y$, alors $\Delta\left(f, \Delta_{Y}\right)=\Delta(f, \Delta(g)) \geq \Delta(f \circ g)$, la différence provenant des diviseurs de $Z$ qui sont $g$-exceptionnels, mais non $f \circ g$-exceptionnels. Sur des modifications adéquates de $Z, X$ et $Y$, on obtient $([\mathrm{Ca} 04,1.31])$ des fibrations $g^{\prime}: Z^{\prime} \rightarrow Y^{\prime}, f^{\prime}: Y^{\prime} \rightarrow X^{\prime}$ telles que $\Delta\left(f^{\prime} \circ g^{\prime}\right)=\Delta\left(f^{\prime}, \Delta\left(g^{\prime}\right)\right)$.

Nous allons généraliser cette relation au cas où l'on a un diviseur orbifolde initial sur $Z$. Les arguments sont cependant essentiellement les mêmes. Pour cela, quelques résultats préliminaires doivent être établis.

Lemme 3.4 Soit $v, f, f^{\prime}=f \circ v$ des applications holomorphes, avec $v$ : $\left(Y^{\prime} \mid \Delta^{\prime}\right) \rightarrow(Y \mid \Delta)$ un morphisme orbifolde biméromorphe tel que $\Delta=v_{*}\left(\Delta^{\prime}\right)$ :

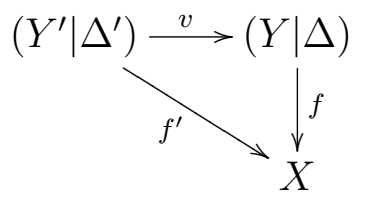

Alors : $\Delta(f, \Delta)=\Delta\left(f^{\prime}, \Delta^{\prime}\right)$.

Démonstration : Soit $D \in W(X)$, alors :

$f^{\prime *}(D)=v^{*}\left(f^{*}(D)\right)=v^{*}\left(\sum_{j} m_{j} . D_{j}+R\right)=\sum_{j} m_{j} \cdot\left(v^{*}\left(D_{j}\right)\right)+v^{*}(R)=$ $\sum_{j} m_{j} \cdot \overline{D_{j}}+\sum_{(j, h)} m_{j} \cdot n_{(j, h)} E_{(j, h)}+v^{*}(R)=\sum_{j} m_{j} \cdot \overline{D_{j}}+R^{\prime}$, où $\overline{D_{j}}$ est le transformé strict de $D_{j}$ par $v$. Bien sûr, $v^{*}(R)$ est $f^{\prime}$-exceptionnel. 
D'autre part, puisque $v$ est un morphisme orbifolde, on a, pour tous $(j, h)$ :

$$
n_{(j, h)} \cdot m^{\prime}\left(E_{(j, h)}\right) \geq m\left(D_{j}\right),
$$

et donc $: n_{(j, h)} \cdot m_{j} \cdot m^{\prime}\left(E_{(j, h)}\right) \geq m_{j} \cdot m\left(D_{j}\right)$. On a noté $m: W(Y) \rightarrow \overline{\mathbb{Q}}$, et $m^{\prime}:$ $W^{\prime}(Y) \rightarrow \overline{\mathbb{Q}}$ les fonctions de multiplicité associées à $\Delta$ et $\Delta^{\prime}$ respectivement.

Puisque $v_{*}\left(\Delta^{\prime}\right)=\Delta$, on a de plus : $m\left(D_{j}\right)=m^{\prime}\left(\overline{D_{j}}\right)$.

Notant $m_{f}$ et $m_{f}^{\prime}$ les multiplicités définissant $\Delta(f, \Delta)$ et $\Delta\left(f^{\prime}, \Delta^{\prime}\right)$ respectivement, on a donc :

$$
m_{f}(D)=i n f_{j}\left\{m_{j} \cdot m\left(D_{j}\right)\right\}
$$

tandis que :

$$
m_{f}^{\prime}(D)=i n f_{(j, h)}\left\{m_{j} \cdot m^{\prime}\left(\overline{D_{j}}\right), m_{j} \cdot n_{(j, h)} \cdot m^{\prime}\left(E_{(j, h)}\right)\right\}
$$

On déduit alors des relations (1) et (2) précédentes que $m_{f}(D)=m_{f}^{\prime}(D)$, ce qui est l'assertion du lemme.

Corollaire 3.5 Dans la situation du lemme 3.4 précédent, si $h: X \rightarrow W$ est une application holomorphe, avec $W$ lisse, alors $\Delta\left(h \circ f^{\prime}, \Delta^{\prime}\right)=\Delta(h \circ f, \Delta)$ est indépendant de $\Delta^{\prime}$, avec $\Delta=v_{*}\left(\Delta^{\prime}\right)$, $v$ étant un morphisme d'orbifoldes géométriques.

Lemme 3.6 Supposons que, dans le diagramme commutatif suivant, $w$ et $g$ soient des morphismes orbifolde, que $w$ et $v$ soient biméromorphes, et que les variétés considérées soient $\mathbb{Q}$-factorielles, connexes et compactes.

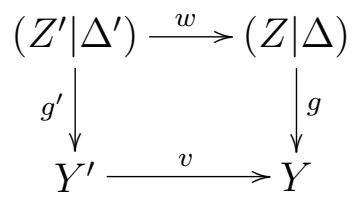

Alors : $v:\left(Y^{\prime} \mid \Delta\left(g^{\prime}, \Delta^{\prime}\right)\right) \rightarrow(Y \mid \Delta(g, \Delta))$ est un morphisme orbifolde. De plus, si $w_{*}\left(\Delta^{\prime}\right)=\Delta$, alors $v_{*}\left(\Delta\left(g^{\prime}, \Delta^{\prime}\right)\right)=\Delta(g, \Delta)$.

Démonstration : Soit $E \in W(Y), E^{\prime} \in W\left(Y^{\prime}\right)$ tels que $v^{*}(E)=s . E^{\prime}+$ $\ldots$, avec $s \geq 1$. On veut montrer que $s . m_{g}^{\prime}\left(E^{\prime}\right) \geq m_{g}(E)$, si $m_{g}, m_{g}^{\prime}$ désignent les fonctions de multiplicité associées à $\Delta(g, \Delta)$ et $\Delta\left(g^{\prime}, \Delta^{\prime}\right)$ respectivement. De même, $m, m^{\prime}$ sont celles de $\Delta$ et $\Delta^{\prime}$.

Si $g^{\prime *}\left(E^{\prime}\right)=\sum_{j} m_{j} \cdot F_{j}^{\prime}+R^{\prime}$, on a $: m_{g}^{\prime}\left(E^{\prime}\right):=\inf _{j}\left\{m_{j} \cdot m^{\prime}\left(F_{j}^{\prime}\right)\right\}$. 
On choisit et fixe un $j$ tel que $g^{\prime}\left(F_{j}^{\prime}\right)=E^{\prime}$ et $m_{j} \cdot m^{\prime}\left(F_{j}^{\prime}\right)=m_{g}^{\prime}\left(E^{\prime}\right)$. On a donc :

$$
\left(v \circ g^{\prime}\right)^{*}(E)=\left(g^{\prime}\right)^{*}\left(s \cdot E^{\prime}+\ldots\right)=s \cdot m_{j} \cdot F_{j}^{\prime}+\ldots
$$

puisque $E^{\prime}$ est la seule composante irréductible de $v^{*}(E)$ contenant $g^{\prime}\left(F_{j}^{\prime}\right)$.

Si $g^{*}(E)=\sum_{k \in K} n_{k} . F_{k}$, on a donc : $m_{g}(E)=\inf _{k}\left\{n_{k} \cdot m\left(F_{k}\right)\right\}$, puisque $g$ est un morphisme orbifolde, par hypothèse.

Posant : $w^{*}\left(F_{k}\right)=s_{(j, k)} \cdot F_{j}^{\prime}+\ldots$, on déduit de (1), en calculant la multiplicité de $F_{j}^{\prime}$ dans $(g \circ w)^{*}(E)$, utilisant $g \circ w=v \circ g^{\prime}$ :

$$
s . m_{j} \geq n_{k} \cdot s_{(j, k)}, \forall k
$$

On déduit de (1) et (2) que :

$$
s . m_{g}^{\prime}\left(E^{\prime}\right)=s \cdot m_{j} \cdot m^{\prime}\left(F_{j}^{\prime}\right) \geq n_{k} \cdot s_{(j, k)} \cdot m^{\prime}\left(F_{j}^{\prime}\right) \geq n_{k} \cdot m\left(F_{k}\right) \geq m_{g}(E),
$$

et donc la conclusion : s. $m_{g}^{\prime}\left(E^{\prime}\right) \geq m_{g}(E)$ par application directe des définitions; l'avant-dernière inégalité résultant du fait que $w$ est un morphisme orbifolde.

La seconde assertion résulte du lemme 3.4

Remarque 3.7 La condition "g morphisme orbifolde" est essentielle. Elle ne peut être affaiblie en : " $g$ " morphisme orbifolde".

\subsection{Fibrations nettes.}

Definition 3.8 Soit $g:(Z \mid \Delta) \rightarrow Y$ une fibration, avec $(Z \mid \Delta)$ et $Y$ lisses. On dira que $g$ est nette (relativement à $g^{\prime}$ ) s'il existe un diagramme commutatif :

dans lequel :

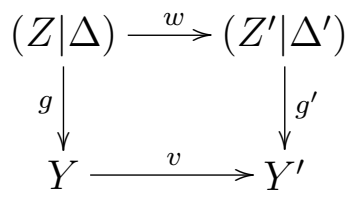

1. $w$ est un morphisme orbifolde, $v$ et $w$ sont biméromorphes, $Z^{\prime}, Y, Y^{\prime}$ lisses, et $w_{*}(\Delta)=\Delta^{\prime}$.

2. Tout diviseur g-exceptionnel de $Z$ est $w$-exceptionnel.

On dira que $g$ est nette si elle est nette relativement à une fibration $g^{\prime}$ comme ci-dessus. 
On dira que $g$ est nette et haute si elle est nette, et si $g:(Z \mid \Delta) \rightarrow$ $(Y \mid \Delta(g, \Delta))$ est un morphisme orbifolde.

On dira que $g$ est strictement nette si elle est nette, et si, de plus, sa base orbifolde est lisse.

Exemple 3.9 Toute fibration sur une courbe est strictement nette et haute.

Proposition 3.10 Si $g^{\prime}:\left(Z^{\prime} \mid \Delta^{\prime}\right) \rightarrow Y^{\prime}$ est donnée, il existe un diagramme comme ci-dessus avec :

1. $g:(Z \mid \Delta) \rightarrow Y$ strictement nette relativement à $g^{\prime}$.

2. $g:(Z \mid \Delta) \rightarrow(Y \mid \Delta(g, \Delta))$ est un morphisme orbifolde (ie : $g$ est strictement nette et haute).

Démonstration : On construit le diagramme commutatif ci-dessous :

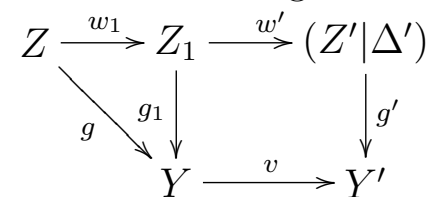

dans lequel $: v$ est une modification telle que le morphisme déduit de $g^{\prime}$ par $v$ soit plat sur la composante pincipale ([R72]), et tel que $Y$ soit lisse, avec $v^{-1}\left(\operatorname{Supp}\left(\Delta\left(g^{\prime}, \Delta^{\prime}\right)\right)\right)$ soit à croisements normaux sur $Y$. La désingularisation de $Z_{1}$ fournit alors $Z$. On pose $: w:=w^{\prime} \circ w_{1}$. Les diviseurs $g$-exceptionnels de $Z$ sont alors $w$-exceptionnels, par platitude de $g_{1}$. On munit $Z$ du diviseur orbifolde $\Delta$ tel que $w_{*}(\Delta)=\Delta^{\prime}$. Pour les diviseurs $F \in W(Z)$ qui sont $w$ exceptionnels, leurs multiplicité peut être choisie arbitrairement assez grande. Ceci établit la première assertion.

La seconde assertion résulte de 3.6, si les multiplicités des diviseurs $g$-exceptionnels de $Z$ (qui sont donc $w$-exceptionnels) sont choisies assez grandes.

Corollaire 3.11 Supposons que, dans le diagramme commutatif suivant, $w$ soit un morphisme orbifolde biméromorphe élémentaire, et que $g$ et $g^{\prime}$ soient des fibrations hautes et strictement nettes, et enfin que $v$ soit biméromorphe.

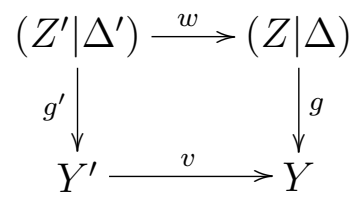

Alors : $v:\left(Y^{\prime} \mid \Delta\left(g^{\prime}, \Delta^{\prime}\right)\right) \rightarrow(Y \mid \Delta(g, \Delta))$ est un morphisme orbifolde biméromorphe élémentaire. 
En particulier, la classe d'équivalence biméromorphe des bases orbifoldes de fibrations équivalentes pour la relation d'équivalence engendrée par de tels diagrammes est bien définie (ie : est indépendante du représentant choisi).

\section{Question 3.12 : Équivalence biméromorphe des bases orbifoldes.}

Soit $(Z \mid \Delta)$ et $\left(Z^{\prime} \mid \Delta^{\prime}\right)$ deux orbifoldes lisses biméromorphiquement équivalentes, avec $Z, Z^{\prime}$ compactes, kähler et connexes. Si $g: Z \rightarrow Y$ et $g^{\prime}: Z^{\prime} \rightarrow Y^{\prime}$ sont deux fibrations biméromorphiquement équivalentes (ie : avec la même famille de fibres génériques), avec $Y, Y^{\prime}$ lisses, et si $g$ et $g^{\prime}$ sont trictement nettes, leurs bases orbifoldes sont-elles biméromorphiquement équivalentes?

Nous établirons dans le chapitre $\S 4$ suivant une propriété plus faible, mais centrale pour les résultats du présent texte : l'égalité de la dimension canonique de deux telles bases orbifoldes.

\subsection{Composition de fibrations}

Dans cette section, toutes les orbifoldes et variétés considérées sont lisses, compactes et connexes.

On considère un diagramme commutatif de morphismes orbifolde entre orbifoldes géométriques lisses (compactes et connexes). On suppose que les flèches verticales sont des fibrations.

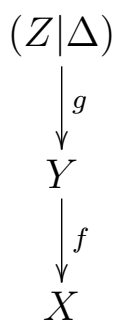

Proposition 3.13 Soit $g: Z \rightarrow Y$ et $f: Y \rightarrow X$ deux fibrations, avec $Y, X$ lisses, et $\Delta$ un diviseur orbifolde sur $Z$.

On peut donc construire trois diviseurs orbifoldes $: \Delta_{Y}:=\Delta(g, \Delta)$ sur $Y$, ainsi que $\Delta_{f g}:=\Delta(f \circ g, \Delta)$ et $\Delta_{f / g}:=\Delta(f, \Delta(g, \Delta))$ sur $X$. Alors :

1. $\Delta(f \circ g, \Delta) \leq \Delta(f, \Delta(g, \Delta))$.

2. On a $\Delta(f \circ g, \Delta)=\Delta(f, \Delta(g, \Delta))$ si $m_{\Delta}(F)$ est assez grand, pour tout $F \in W(Z)$ qui est $g$-exceptionnel, mais non pas $f \circ g$-exceptionnel.

3. Si $g:(Z \mid \Delta) \rightarrow(Y \mid \Delta(g, \Delta))$ est un morphisme orbifolde, on $a: \Delta(f \circ$ $g, \Delta)=\Delta(f, \Delta(g, \Delta))$. 
Démonstration : Soit $D \in W(X)$. Alors $\left(g^{*}\left(f^{*}\right)\right)(D)=\left(\sum_{j} g^{*}\left(m_{j} E_{j}\right)+\right.$ $g^{*}(R)=\sum_{j, h} m_{j} . n_{j, h} F_{j, h}+R^{\prime}$. Notons $m_{f g}$ la multiplicité de $\Delta(f \circ g, \Delta)$, et $m_{f / g}$ celle de $\Delta(f, \Delta(g, \Delta))$.

Donc : $m_{f g}(D):=\inf _{j, h}\left\{m_{j} . n_{j, h} \cdot m_{\Delta}\left(F_{j, h}\right)\right\}$. La somme porte sur les composantes $F_{j, h}$ de $(f \circ g)^{*}(D)$ qui ne sont pas $f \circ g$-exceptionnelles.

Par définition, $m_{f / g}(D)=\inf _{j, h}\left\{m_{j} . n_{j, h} . m_{\Delta}\left(F_{j, h}\right)\right\}$, la somme portant sur les composantes de $(f \circ g)^{*}(D)$ qui ne sont exceptionnelles ni pour $g$, ni pour $f \circ g$.

D'où la première assertion.

Pour établir la seconde assertion, il suffit donc d'observer que $m_{f g}(D)=$ $m_{f / g}(D)$ si, pour chaque $F$ qui est une composante $g$-exceptionnelle mais non $f \circ g$-exceptionnelle de de $(f \circ g)^{*}(D)$, on a $: m_{\Delta}(F) \geq m_{j} . n_{j, h} . m_{\Delta}\left(F_{j, h}\right)$, pour (au moins) une composante $F_{j, h}=F^{\prime}$ qui n'est ni $g$-, ni $f \circ g$-exceptionnelle, et telle que, de plus, $g(F) \subset g\left(F^{\prime}\right)=g\left(F_{j, h}\right)=D_{j}=D$ (et il en existe toujours).

Pour établir la troisième assertion, nous allons, pour tout diviseur $F \in$ $W(Z)$ qui est $g$-exceptionnel, mais non $f \circ g$-exceptionnel, montrer l'existence d'un tel diviseur $F^{\prime}$, si $g$ est un morphisme orbifolde.

Soit donc $D:=f(g(F)) \in W(X)$. Soit $E \in W(Y)$ tel que $g^{*}(E)=$ $t_{E, F} \cdot F+\ldots$, avec $t_{E, F}>0$.

Puisque $g:(Z \mid \Delta) \rightarrow\left(Y \mid \Delta_{Y}\right)$ est un morphisme orbifolde, on a :

$$
t_{E, F} \cdot m_{\Delta}(F) \geq m_{g}(E):=m_{\Delta_{Y}}(E) .
$$

De plus, il existe $F^{\prime} \in W(Z)$ tel que $g\left(F^{\prime}\right)=E$, et $t_{E, F^{\prime}} \cdot m_{\Delta}\left(F^{\prime}\right)=m_{g}(E)$, avec $g^{*}(E)=t_{E, F^{\prime}} \cdot F^{\prime}+\ldots$, par définition de $\Delta_{Y}=\Delta(g, \Delta)$. On a donc aussi : $t_{E, F} \cdot m_{\Delta}(F) \geq t_{E, F^{\prime}} \cdot m_{\Delta}\left(F^{\prime}\right)$.

Soit $D:=f \circ g(F)=f \circ g\left(F^{\prime}\right)$. Si $f^{*}(D)=s . E+\ldots$, on a donc : $(f \circ g)^{*}(D)=s \cdot t_{E, F^{\prime}} \cdot F^{\prime}+t^{+} \cdot F+\ldots$, où $t^{+} \geq s . t_{E, F}$, puisque $f^{*}(D)$ peut avoir un support dont plusieurs composantes distinctes contiennent $g(F)$. Il en résulte que $t^{+} \cdot m_{\Delta}(F) \geq s \cdot t_{E, F^{\prime}} \cdot m_{\Delta}\left(F^{\prime}\right) \geq s \cdot m_{g}(E) \geq m_{f / g}(D)$. Puisque ceci est vrai pour tout $F \in W(Z)$ qui est $g$-exceptionnel, mais non $f \circ g$ exceptionnel, on a bien : $m_{f \circ g}(D) \geq m_{f / g}(D), \forall D \in W(X)$. En effet, $m_{f / g}(D)$ est le minimum des quantités s.t $t_{E, F^{\prime}} \cdot m_{\Delta}\left(F^{\prime}\right)$, pour $F^{\prime}$ tel que $g\left(F^{\prime}\right)=E^{\prime}$, avec $f\left(E^{\prime}\right)=D$.

De 3.13, on déduit maintenant :

Proposition 3.14 Soit $g: Z \rightarrow Y$ et $f: Y \rightarrow X$ deux fibrations. Soit $\Delta$ un diviseur orbifolde sur $Z$. Si $g:(Z \mid \Delta) \rightarrow Y$ est nette et haute (ce 
que l'on peut réaliser par un morphisme biméromorphe élémentaire), alors $\Delta(f \circ g, \Delta)=\Delta(f, \Delta(g, \Delta))$.

Ce résultat montre que, quitte à effectuer sur $(Z \mid \Delta)$ une transformation biméromorphe élémentaire, on peut calculer la base orbifolde de $f \circ g$ en deux étapes.

De 3.13 et 3.10, on déduit plus généralement, par récurrence sur $r$ :

Proposition 3.15 Soit $g_{j}^{\prime}: Z_{j}^{\prime} \rightarrow Z_{j+1}^{\prime}$, pour $j=0,1, \ldots, r$ des fibrations méromorphes dominantes connexes, avec $Z_{0}^{\prime}$ lisse, compacte et connexe. Soit $\Delta_{0}^{\prime}$ un diviseur orbifolde sur $Z_{0}^{\prime}$. Il existe alors un morphisme orbifolde biméromorphe élémentaire $w:\left(Z_{0} \mid \Delta_{0}\right) \rightarrow\left(Z_{0}^{\prime} \mid \Delta_{0}^{\prime}\right)$, des applications biméromorphes $w_{j}: Z_{j} \rightarrow Z_{j}^{\prime}$, et des applications holomorphes $g_{j}: Z_{j} \rightarrow Z_{j+1}$ telles que, pour $j=0,1, \ldots, r$ :

1. $g_{j}^{\prime} \circ w_{j}=w_{j+1} \circ g_{j}$.

2. $h_{j}:=g_{j} \circ g_{j-1} \circ \cdots \circ g_{1} \circ g_{0}: Z_{0} \rightarrow Z_{j+1}$ est strictement nette et haute.

3. $\Delta\left(h_{j}, \Delta_{0}\right)=\Delta\left(g_{j}, \Delta\left(h_{j-1}, \Delta_{0}\right)\right)$.

\subsection{Fibres orbifoldes.}

Definition 3.16 Soit $f:(X \mid \Delta) \rightarrow Y$ une fibration (propre). On suppose l'orbifolde géométrique $(X \mid \Delta)$ lisse. On définit alors la fibre générique orbifolde $(X \mid \Delta)_{y}:=\left(X_{y} \mid \Delta_{y}\right)$ comme étant, pour $y \in Y$ générique, la restriction de $\Delta \grave{a} X_{y}:=f^{-1}(y)$. C'est-à-dire que si $\Delta=\sum_{D \in W(X)}\left(1-\frac{1}{m_{\Delta}(D)}\right)$. D, on $a: \Delta_{y}:=\sum_{D \in W(X)}\left(1-\frac{1}{m_{\Delta}(D)}\right) \cdot\left(D \cap X_{y}\right)$.

Le théorème de Sard montre alors que $\left(X_{y} \mid \Delta_{y}\right)$ est lisse.

Definition 3.17 On dit que $f$ et $f^{\prime}$ sont (biméromorphiquement) élémentairement équivalentes s'il existe un diagramme commutatif :

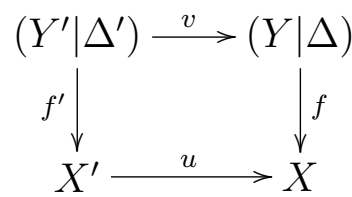

dans lequel $u, v$ sont biméromorphes, et $v$ un morphisme orbifolde tel que $v_{*}\left(\Delta^{\prime}\right)=\Delta$.

Plus généralement, $f$ et $f^{\prime}$ sont équivalentes (on note alors : $f \sim f^{\prime}$ ) si elles le sont pour la relation d'équivalence engendrée par de tels diagrammes. 
Proposition 3.18 Si $f:(X \mid \Delta) \rightarrow Y$ et $f^{\prime}:\left(X^{\prime} \mid \Delta^{\prime}\right) \rightarrow Y^{\prime}$ sont deux fibrations biméromorphiquement équivalentes, et si $(X \mid \Delta)$ et $(X \mid \Delta)$ sont lisses, leurs fibres orbifoldes génériques correspondantes sont biméromorphiquement équivalentes.

Démonstration. Il suffit de démontrer l'assertion lorsque $f$ et $f^{\prime}$ sont élémentairement équivalentes. Dans ce cas, il suffit de choisir $x \in X$ tel que les fibres orbifoldes de $f$ et $f^{\prime}$ au-dessus de $x$ soient lisses. Alors $v$ induit une équivalence orbifolde élémentaire entre ces deux fibres

Remarque 3.19 Lorsque $f$ n'est pas holomorphe, l'assertion précédente peut être en défaut : les fibres orbifoldes génériques de 2 modèles biméromorphes (au sens de 3.17 ci-dessus) de $(X \mid \Delta)$ rendant $f$ holomorphe ne sont pas nécessairement biméromorphes, et peuvent même avoir des dimensions canoniques différentes. Voir l'exemple 3.20 ci-dessous, mais aussi le théorème 6.3 pour une situation dans laquelle la dimension canonique des fibres orbifoldes génériques ne dépend pas du modèle biméromorphe choisi.

Remarquons que l'exemple simple qui suit montre que la proposition 3.18 tomberait en défaut si la relation d'équivalence biméromorphe ci-dessus n'imposait pas l'holomorphie et la préservation des fibres de $f$.

Remarque 3.20 Par contre, si f n'est pas presque-holomorphe ${ }^{13}$, la fibre orbifolde générique $\left(X_{y} \mid \Delta_{X_{y}}\right)$ de $f$ n'est pas bien définie, à équivalence orbifolde près, et sa dimension canonique dépend en général du modèle biméromorphe choisi. Considérer, par exemple, $f: X:=\mathbb{P}^{2} \rightarrow \mathbb{P}^{1}$ la fibration définie par les coniques passant par 4 points $a_{j} \in \mathbb{P}^{2}$ en position générale. Les fibres de cette fibrations sont donc les coniques de la famille, sans structure orbifolde. Si l'on éclate les points $a_{j}$, et si l'on dénote par $A_{j}$ les diviseurs exceptionnels de cet éclatement, alors la fibration holomorphe $f^{\prime}: X^{\prime} \rightarrow \mathbb{P}^{1}$ obtenue est holomorphe. Munissant $X^{\prime}$ de la structure orbifolde $\Delta_{m}^{\prime}:=\sum_{j}(1-1 / m) . A_{j}$, les fibres lisses de $f^{\prime}$ rencontrent transversalement les $A_{j}$, et sont donc munies de la structure orbifolde induite consistant en quatre points munis de la multiplicité $m$, et leur dimension canonique est donc $-\infty$ (resp. 0, resp. 1) si $m=1$ (resp. $m=2$, resp. $m \geq 3$ ). Observer que, pour tout $m,(X \mid 0)$ et $\left(X^{\prime} \mid \Delta_{m}^{\prime}\right)$ sont biméromorphes.

\footnotetext{
${ }^{13}$ Voir définition 7.16 .
} 


\section{DIMENSION CANONIQUE D'UNE FI- BRATION.}

\subsection{Dimension canonique d'une fibration.}

Definition 4.1 Soit $f:(Y \mid \Delta) \rightarrow X$ une fibration avec $(Y \mid \Delta)$ et $X$ lisses. On définit : $L_{f}:=f^{*}\left(K_{X}\right) \subset \Omega_{Y}^{p}$, si $p:=\operatorname{dim}(X)$.

Rappelons que l'on a aussi défini dans le lemme 2.16 les invariants suivants :

$\kappa\left(Y \mid \Delta, L_{f}\right):=\kappa(f, \Delta)$, et $p_{N}\left(Y \mid \Delta, L_{f}\right):=p_{N}(f, \Delta), \forall N>0$.

Remarque 4.2 Soit $v:\left(Y^{\prime} \mid \Delta^{\prime}\right) \rightarrow(Y \mid \Delta)$ un morphisme orbifolde biméromorphe entre orbifoldes lisses tel que $v_{*}\left(\Delta^{\prime}\right)=\Delta$, et $f^{\prime}:=f \circ v$. Alors il résulte du lemme 3.4 que : $p_{N}\left(f^{\prime}, \Delta^{\prime}\right)=p_{N}(f, \Delta), \forall N>0$, et $\kappa(f, \Delta)=\kappa\left(f^{\prime}, \Delta^{\prime}\right)$.

L'objectif de cette section est de démontrer le :

Théorème 4.3 Soit $f:(Y \mid \Delta) \rightarrow X$ une fibration, avec $(Y \mid \Delta)$ et $X$ lisses. Alors :

1. $\kappa\left(Y \mid \Delta, L_{f}\right) \leq \kappa(X \mid \Delta(f, \Delta))$.

2. Si $f$ est nette, la dernière inégalité est une égalité.

Démonstration : Elle utilise le lemme 4.5 ci-dessous.

Definition 4.4 Soit $F$ un diviseur entier effectif sur $Y$. On dit que $F$ est partiellement supporté sur les fibres de $f$ s'il existe un diviseur effectif $D$ sur $X$ tel que $F \leq f^{*}(D)$, et tel que pour toute composante irréductible $D^{\prime}$ de $D$, il existe une composante irréductible $E^{\prime}$ de $f^{-1}\left(D^{\prime}\right)$ non contenue dans $F$, et telle que $f\left(E^{\prime}\right)=D^{\prime}$.

On a :

Lemme 4.5 ([Ca04, lemma 1.22]) Soit $F$ un diviseur de $Y$ partiellement supporté sur les fibres de $f$, et $L \in \operatorname{Pic}(X)$.

Alors l'injection naturelle : $H^{0}\left(Y, f^{*}(L)\right) \subset H^{0}\left(Y, f^{*}(L)+F\right)$ est surjective.

Le théorème 4.3 résulte alors de la proposition 4.6 suivante : 
Proposition 4.6 Soit $N_{0}=N_{0}(f, \Delta)$ le plus petit commun multiple des numérateurs des multiplicités finies de $\Delta(f, \Delta):=\Delta_{f}$. Soit $N:=N_{0} . M$, et $\overline{L_{N}}:=\overline{L_{N_{0} . M}} \subset S_{N, p}(Y \mid \Delta), \forall M>0, p:=\operatorname{dim}(X)$. Alors :

1. Il existe des diviseurs effectifs (dépendants de $M$ ) $F, E^{+}, E^{-}$sur $Y$ tels que : $\overline{L_{N}}=f^{*}\left(N\left(K_{X}+\Delta_{f}\right)+F+E^{+}-E^{-}\right.$, et tels que : $F$ soit partiellement supporté sur les fibres de $f, E^{+}$et $E^{-}$sont $f$-exceptionnels et sans composante commune.

2. Si f est nette, il existe un isomorphisme naturel :

$$
j_{M}: H^{0}\left(Y, \overline{L_{N}}\right) \rightarrow f^{*}\left(H^{0}\left(X,\left(N\left(K_{X}+\Delta_{f}\right)\right)\right)\right) .
$$

\section{Démonstration (de 4.6) :}

Assertion 1. Il suffit de voir que, en codimension 1 au-dessus de $X$, on a : $\overline{L_{N}}=f^{*}\left(N .\left(K_{X}+\Delta_{f}\right)+F\right.$, et même seulement que, si $D \in W(X)$, et $E \in W(Y)$ sont tels que $f(E)=D$, alors au-dessus du point générique de $D$, on a égalité entre ces deux faisceaux (pour un choix convenable de $F$ déterminé ci-dessous).

On note $\Delta_{f}:=\Delta(f, \Delta), m: W(Y) \rightarrow \overline{\mathbb{Q}^{+}}$la multiplicité définissant $\Delta$, et $m_{f}$ celle définissant $\Delta_{f}$.

Si $f^{*}(D)=t . E+\ldots$, avec $t>0$ entier, alors $t . s:=t . m(E) \geq m_{f}(D):=r$, par hypothèse. Dans des coordonnées locales $y=\left(y_{1}, \ldots, y_{n}\right)$ adaptées au voisinage du point $b \in E$, générique dans $E, E$ est défini par l'équation $y_{1}=0$, et $f(y)=\left(x_{1}, \ldots, x_{p}\right)=\left(y_{1}^{t}, f_{2}, \ldots, f_{p}\right)$, avec $w^{\prime}(b):=d f_{2}(b) \wedge \cdots \wedge d f_{p}(b) \neq 0$. De plus, $D$ est défini par l'équation $x_{1}=0$. Enfin, $\Delta$ (resp. $\Delta_{f}$ ) a en $b$ (resp. en $a:=f(b)$ ) pour équation $: y_{1}^{1-1 / s}=0$ (resp. $x_{1}^{1-1 / r}=0$ (avec $s, r$ définis ci-dessus, et $s . t \geq r)$.

Un générateur de $N .\left(K_{X}+\Delta_{f}\right)$ en $a$ est donc : $w:=\left(d x_{1} \wedge \cdots \wedge\right.$ $\left.d x_{p}\right) / x_{1}^{N-N / r}$. On en déduit que $f^{*}(w)=y_{1}^{((t / r)-s) N-N} \cdot\left(d y_{1} \wedge w^{\prime}\right)^{\otimes N}$, où $w^{\prime}$ est une $(p-1)$-forme holomorphe non nulle et sans zéro sur $Y$ près de $b$. On a, par hypothèse, $t / r \geq 1 / s$, avec égalité pour au moins l'une de composantes $E$ de $f^{*}(D)$. On en déduit bien la première assertion.

Assertion 2. On suppose que l'on a un diagramme commutatif :

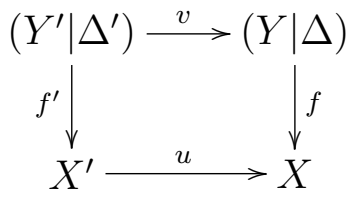

dans lequel les flèches horizontales sont biméromorphes, les orbifoldes lisses, et que les diviseurs $f^{\prime}$-exceptionnels de $Y^{\prime}$ sont $v$-exceptionnels. Donc 
$f^{\prime}$ est nette (relativement à $f$ ), et on veut montrer l'assertion 2 pour $f^{\prime}$ (et non pas pour $f$ ). On note $m^{\prime}, \Delta_{f}^{\prime}=\Delta_{f^{\prime}}^{\prime}, F^{\prime}, \ldots$ les données relatives à $f^{\prime}$ qui sont analogues à celles introduites pour $f$.

Soit $A \subset Y$ le lieu au-dessus duquel $v$ n'est pas un isomorphisme : il est analytique de codimension au moins deux dans $Y$. Observons tout d'abord que l'on a une injection naturelle de faisceaux $v_{*}\left(f^{\prime *}\left(N\left(K_{X^{\prime}}+\Delta_{f}^{\prime}\right)\right)+F^{\prime}\right) \rightarrow$ $\left(f^{\prime *}\left(N\left(K_{X}+\Delta_{f}\right)\right)+F\right)$ qui est un isomorphisme au-dessus de $(Y-A)$. Pour tout diviseur $v$-exceptionnel $E^{\prime}$ (pas nécessairement effectif) de $Y^{\prime}$, on a donc une bijection naturelle : $H^{0}\left(Y^{\prime}, N\left(K_{X^{\prime}}+\Delta_{f}^{\prime}\right)+F^{\prime}+E^{\prime}\right) \cong H^{0}\left(Y^{\prime}, N\left(K_{X^{\prime}}+\right.\right.$ $\left.\left.\Delta_{f}^{\prime}\right)+F^{\prime}\right)$, puisque les sections ainsi définies peuvent être vues comme des sections de $f^{*}\left(N\left(K_{X} \mid \Delta_{f}\right)\right)$ sur $(Y-A)$, la conclusion résultant du théorème d'Hartogs.

On a donc, en particulier, un isomorphisme : $H^{0}\left(Y^{\prime}, f^{\prime *}\left(N\left(K_{X^{\prime}}+\Delta_{f}^{\prime}\right)+\right.\right.$ $\left.F^{\prime}\right) \cong H^{0}\left(Y^{\prime}, f^{\prime *}\left(N\left(K_{X^{\prime}}+\Delta_{f}^{\prime}\right)\right)+F^{\prime}+E^{\prime+}-E^{\prime-}\right) \cong H^{0}\left(Y^{\prime}, \overline{L_{f^{\prime}, N}}\right)$.

Par le lemme 4.5, on a donc: $H^{0}\left(Y^{\prime}, f^{\prime *}\left(N\left(K_{X^{\prime}}+\Delta_{f}^{\prime}\right)\right)+F^{\prime}\right) \cong$ $H^{0}\left(Y^{\prime}, f^{\prime *}\left(N\left(K_{X^{\prime}}+\Delta_{f}^{\prime}\right)\right)\right) \cong H^{0}\left(Y^{\prime}, \overline{L_{f^{\prime}, N}}\right)$, et la conclusion

\section{2 Équivalence biméromorphe de fibrations.}

Soit $f:(Y \mid \Delta) \rightarrow X$, et $f:\left(Y^{\prime} \mid \Delta^{\prime}\right) \rightarrow X^{\prime}$ des fibrations, avec $X, Y, X^{\prime}, Y^{\prime}$ lisses compactes et connexes.

- On supposera dans tout ce $\S 4.2$ que les orbifoldes géométriques $(Y \mid \Delta)$ et $\left(Y^{\prime} \mid \Delta^{\prime}\right)$ sont lisses.

Rappelons (3.17) la :

Definition 4.7 On dit que $f$ et $f^{\prime}$ sont (biméromorphiquement) élémentairement équivalentes s'il existe un diagramme commutatif :

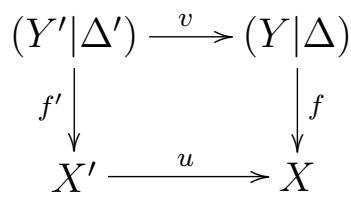

dans lequel $u, v$ sont biméromorphes, $u$ holomorphe mais $v$ seulement méromorphe, et $v$ un morphisme orbifolde tel que $v_{*}\left(\Delta^{\prime}\right)=\Delta$.

Plus généralement, $f$ et $f^{\prime}$ sont équivalentes (on note alors : $f \sim f^{\prime}$ ) si elles le sont pour la relation d'équivalence engendrée par de tels diagrammes.

Remarque 4.8 En général, u n'est pas un morphisme orbifolde sur les bases orbifoldes de $f$ et $f^{\prime}$, même s'il est holomorphe. 
Proposition 4.9 Dans le diagramme précédent, supposons u holomorphe. Aors :

$$
\begin{aligned}
& \text { 1. } u_{*}\left(\Delta\left(f^{\prime}, \Delta^{\prime}\right)\right)=\Delta(f, \Delta) \\
& \text { 2. } \kappa(X \mid \Delta(f, \Delta)) \geq \kappa\left(X^{\prime} \mid \Delta\left(f^{\prime}, \Delta\right)\right) \\
& \text { 3. On a égalité si } \kappa(X) \geq \kappa\left(X^{\prime}\right) \geq 0
\end{aligned}
$$

Démonstration : La première assertion résulte du lemme 3.6. La seconde s'ensuit immédiatement. La troisième est établie dans [Ca04,1.14,p. 514].

- La dimension canonique de la base d'une fibration n'est pas, en général, un invariant biméromorphe. On va maintenant attacher à une fibration $f$ : $(Y \mid \Delta) \rightarrow X$ comme ci-dessus un invariant biméromorphe (dans la catégorie des orbifoldes lisses) fondamental d'une fibration, grâce à la propriété de décroissance ci-dessus.

Definition 4.10 Soit $f:(Y \mid \Delta) \rightarrow X$ une fibration avec $(Y \mid \Delta)$ et $X$ lisses. On définit : $\kappa(X, f \mid \Delta):=\inf _{f^{\prime} \sim f}\left\{\kappa\left(X^{\prime} \mid \Delta(f, \Delta)\right\}\right.$. C'est la dimension canonique d'une fibration orbifolde.

Corollaire 4.11 Soit $f:(Y \mid \Delta) \rightarrow X$ une fibration, avec $(Y \mid \Delta)$ et $X$ lisses. Alors :

1. $\kappa\left((Y \mid \Delta), L_{f}\right)=\kappa\left(\left(Y^{\prime} \mid \Delta^{\prime}\right), L_{f^{\prime}}\right)$ si $f \sim f^{\prime}$.

2. $\kappa(f \mid \Delta)=\kappa\left((Y \mid \Delta), L_{f}\right) \leq \kappa(X \mid \Delta(f, \Delta))$.

3. Si $f$ est nette, la dernière inégalité est une égalité.

Démonstration : La première assertion résulte de l'invariance biméromorphe de la dimension canonique de $\left(L_{f}, \Delta\right)$, par le théorème 2.19.

Pour l'assertion 2, on choisit un diagramme :

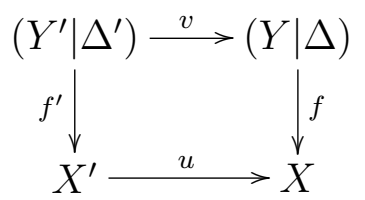

comme ci-dessus, avec $f^{\prime}$ nette relativement à $f, f$ choisie telle que $\kappa(f \mid \Delta)=\kappa(X \mid \Delta(f, \Delta))=\kappa\left(X^{\prime} \mid \Delta\left(f^{\prime}, \Delta^{\prime}\right)\right)$, la première égalité par le choix de $f$, la seconde par la décroissance 4.9 .2 et minimalité de $\kappa(X \mid \Delta(f, \Delta))$. Puisque $f^{\prime}$ est nette, on a : $\kappa\left(X^{\prime} \mid \Delta\left(f^{\prime}, \Delta^{\prime}\right)\right)=\kappa\left(\left(Y^{\prime} \mid \Delta^{\prime}\right), L_{f^{\prime}}\right)$, par le théorème 4.3. Puisque $\kappa\left(\left(Y^{\prime} \mid \Delta^{\prime}\right), L_{f^{\prime}}\right)=\kappa\left((Y \mid \Delta), L_{f}\right)$, par invariance birationnelle, on a : $\kappa(f \mid \Delta)=\kappa\left(X^{\prime} \mid \Delta\left(f^{\prime}, \Delta^{\prime}\right)\right)=\kappa\left(\left(Y^{\prime} \mid \Delta^{\prime}\right), L_{f^{\prime}}\right)=\kappa\left((Y \mid \Delta), L_{f}\right)$. L'inégalité résulte de ce que $\kappa\left(X^{\prime} \mid \Delta\left(f^{\prime}, \Delta^{\prime}\right)\right) \leq \kappa(X \mid \Delta(f, \Delta))$, par 4.9

L'assertion 3 n'est autre que 4.11 
Corollaire 4.12 Soit un diagramme commutatif de morphismes orbifoldes :

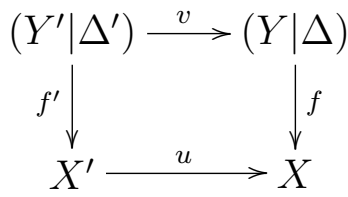

dans lequel les flèches horizontales sont génériquement finies, les flèches verticales des fibrations, avec $Y^{\prime}\left|\Delta^{\prime}, Y\right| \Delta, X^{\prime}, X$ lisses. Alors :

1. $\kappa\left(f \circ v \mid \Delta^{\prime}\right) \geq \kappa(f \mid \Delta)$

2. Si $v$ est étale en codimension 1 , on a égalité.

Démonstration : Il suffit, par 4.3 pour l'assertion 1, et 2.22 pour l'assertion 2, de montrer (par unicité de la saturation) que $v^{*}\left(L_{f}\right)=L_{f^{\prime}} \subset$ $\Omega_{Y^{\prime}}^{p}, p:=\operatorname{dim}(X)$ au point générique de $Y^{\prime}$. Or ceci est évident, puisqu'en un tel point : $v^{*}\left(L_{f}\right)=(f \circ v)^{*}\left(K_{X}\right)=f^{\prime *}\left(u^{*}\left(K_{X}\right)\right)=f^{\prime *}\left(K_{X^{\prime}}\right) \bullet$

Remarque 4.13 Les corollaires 4.6 et 4.12 (et leurs démonstrations) subsistent lorsque $X^{\prime}, X$ sont seulement normaux, et $f, f^{\prime}$ méromorphes, pourvu que l'on définisse $L_{f}, L_{f^{\prime}}$ comme les images dans $\Omega_{Y}^{p}, \Omega_{Y^{\prime}}^{p}$ de fibrés canoniques de modèles lisses arbitraires.

De 4.3 on déduit que :

Corollaire 4.14 Si $p:=\operatorname{dim}(X)$, si $f \sim f^{\prime}$ sont comme ci-dessus ${ }^{14}$, alors $\kappa(f \mid \Delta)=\kappa\left(f^{\prime} \mid \Delta^{\prime}\right):=\kappa\left((Y \mid \Delta), L_{f}\right)$ sont bien définis, où $L_{f} \subset \Omega_{Y}^{p}$ est cohérent de rang 1 , et coincide avec $f^{*}\left(K_{X}\right)$ aux points génériques de $Y$ (en lesquels $f$ est régulière).

En particulier, si $f:(X \mid \Delta) \rightarrow Y$ est une fibration méromorphe dominante, $\kappa(f \mid \Delta)$ est bien définie, sur tout modèle holomorphe net de $f$, et est indépendante de ce modèle. On l'appelle la dimension canonique de $f$.

On notera $[Y \mid \Delta(f \mid \Delta)]$ la base orbifolde d'une tel modèle biméromorphe strictement net et haut de $f$. On l'appellera une base orbifolde stable de $(f \mid \Delta)$.

Il peut sembler inattendu que la dimension canonique soit bien définie, même pour les fibrations non presque-holomorphes, puisque la dimension canonique de la fibre orbifolde générique n'est pas un invariant biméromorphe. Une explication partielle est fournie par la propriété suivante, puisque les composantes orbifoldes non invariantes biméromorphiquement sont horizontales dans le sens ci-dessous.

${ }^{14}$ Voir la définition 3.17. 
Corollaire 4.15 Soit $f:(Y \mid \Delta) \rightarrow X$ une fibration holomorphe nette, avec $(Y \mid \Delta)$ une orbifolde géométrique lisse. Soit $\Delta^{\text {vert }}\left(\right.$ resp. $\left.\Delta^{\text {hor }}\right)$ le diviseur orbifolde déduit de $\Delta$ par suppression de ses composantes horizontales (resp. verticales), c'est-à-dire celles qui se projettent surjectivement (resp. ne se projettent pas surjectivement) sur $X$ par $f$.

$$
\text { Alors : } \kappa(f \mid \Delta)=\kappa\left(f \mid \Delta^{\text {vert }}\right) \text {. }
$$

Démonstation : Par construction, $\Delta(f, \Delta)=\Delta\left(f, \Delta^{\text {vert }}\right)$. Puisque $f$ est nette, $\left.\kappa(f \mid \Delta)=\kappa(X \mid \Delta(f, \Delta))=\kappa\left(X \mid \Delta\left(f, \Delta^{\text {vert }}\right)\right)=\kappa\left(f \mid \Delta^{\text {vert }}\right)\right)$

\subsection{Fibration de Moishezon-Iitaka.}

Soit $(X \mid \Delta)$ une orbifolde géométrique lisse telle que $\kappa(X \mid \Delta) \geq 0$. On suppose $X$ compacte et connexe. Pour tout entier $m>0$ tel que $m \Delta$ soit entier et $H^{0}\left(X, m\left(K_{X}+\Delta\right)\right)$ soit non nul, on note $\Phi_{m}: X \rightarrow \mathbb{P}_{N_{m}}$ l'application méromorphe associée à ce système linéaire.

Les arguments classiques (voir [U75], par exemple), montrent que pour $m$ assez grand et divisible, cette application est à fibres connexes, indépendantes à biméromorphie près de $m$, et que ces fibres orbifoldes stables génériques ont $\kappa=0$, et que la base de cette fibration est de dimension $\kappa(X \mid \Delta)$.

On la notera $M_{(X \mid \Delta)}:(X \mid \Delta) \rightarrow M(X \mid \Delta)$, et on l'appellera la fibration de Moishezon-Iitaka ${ }^{15}$ de $(X \mid \Delta)$.

Elle peut être caractérisée, avec les mêmes arguments que dans le cas non-orbifolde, comme suit : toute fibration $g:(X \mid \Delta) \rightarrow Y$ dont les fibres orbifolde $F$ générales satisfont $\kappa(F)=0$ domine $M_{\left(X \mid \Delta_{X}\right)}$.

On en déduit immédiatemment la propriété de fonctorialité suivante :

Lemme 4.16 Soit $f:\left(X \mid \Delta_{X}\right) \rightarrow\left(Y \mid \Delta_{Y}\right)$ un morphisme dans la catégorie méromorphe des orbifoldes géométriques lisses ${ }^{16}$. On suppose que $X$ est compacte et connexe, que $\kappa\left(X \mid \Delta_{X}\right) \geq 0$, et que $\kappa\left(Y \mid \Delta_{Y}\right) \geq 0$.

Notons (pour simplifier les notations) $\left[M_{X} \mid \Delta_{M_{X}}\right]$ et $\left[M_{Y} \mid \Delta_{M_{Y}}\right]$ des bases orbifoldes stables de $M_{\left(X \mid \Delta_{X}\right)}$ et de $M_{\left(Y \mid \Delta_{Y}\right)}$ respectivement.

Il existe alors un morphisme (dans la catégorie biméromorphe, sur des représentants adéquats) $M_{f}:\left[M_{X} \mid \Delta_{M_{X}}\right] \rightarrow\left[M_{Y} \mid \Delta_{M_{Y}}\right]$ tel que : $M_{f} \circ$ $M_{\left(X \mid \Delta_{X}\right)}=M_{\left(Y \mid \Delta_{Y}\right)} \circ f$.

\footnotetext{
${ }^{15}$ Appelée habituellement "fibration d'Iitaka", bien qu'introduite indépendamment par B. Moishezon au CIM de Nice (1970).

${ }^{16}$ ie : $f$ devient un morphisme orbifolde sur des représentants biméromorphes adéquats de $\left(X \mid \Delta_{X}\right)$ et $\left(Y \mid \Delta_{Y}\right)$.
} 


\subsection{Fibrations de type général et orbifoldes spéciales.}

On définit ces deux notions, utilisées constamment dans la suite.

Definition 4.17 Soit $f:(Y \mid \Delta) \rightarrow X$ une fibration méromorphe avec $X, Y$ compacts et iréductibles, et $(Y \mid \Delta)$ une orbifolde géométrique lisse. On dit que $(f \mid \Delta)$ (ou $f$ s'il n' y a pas d'ambiguité sur $\Delta$ ) est une fibration de type général si $\kappa(f \mid \Delta)=\operatorname{dim}(Y)>0$.

Cet ensemble ne dépend donc que de la classe d'équivalence biméromorphe de $(Y \mid \Delta)$.

- La notion d'orbifolde géométrique spéciale est antithétique de celle de fibration de type général.

Definition 4.18 Une orbifolde géométrique lisse $(Y \mid \Delta)$, avec $Y$ compacte et connexe est dite spéciale si :

1. $Y \in \mathcal{C}$ (ie : $Y$ est biméromorphe à une variété Kählérienne compacte).

2. Il n'existe pas de fibration $f:(Y \mid \Delta) \rightarrow X$ de type général. (De manière équivalente : $(Y \mid \Delta)$ n'a pas d'application méromorphe dominante "stable" sur une orbifolde géométrique de type général de dimension strictement positive. Cette notion se formule donc naturellement dans la catégorie biméromorphe des orbifoldes géométriques). 


\section{COURBES RATIONNELLES ORBI- FOLDES.}

Nous introduisons ici les notions de base de la géométrie des courbes rationnelles dans le contexte orbifolde (lisse), et posons un certain nombre de questions, analogues orbifolde de celles déjà connues ou conjecturées dans le cadre non-orbifolde. Nous montrons enfin que lorsque $(X \mid \Delta)=X^{\prime} / G$ est un quotient global (voir la définition 5.15) ces propriétés peuvent être déduites des résultats connus lorsque $\Delta=0$. On montre en effet que, dans ce cas très particulier, on peut "descendre" (5.6), mais aussi "relever" (5.16) les courbes rationnelles à un revêtement orbifolde étale sur lequel $\Delta=0$. On espère pouvoir étendre ces résultats au cas général (où $(X \mid \Delta)$ est lisse et entière) en remplaçant le revêtement orbifolde $X^{\prime}$ dans les démonstrations par le champ algébrique lisse de Deligne-Mumford associé à $(X \mid \Delta)$.

\subsection{Notion de courbe rationnelle orbifolde}

Soit $(X \mid \Delta)$ une orbifolde lisse.

- Si les multiplicités sont entières ou $+\infty$, les morphismes orbifoldes considérés peuvent être de deux types :

1. Soit divisibles (ou encore : classiques). On notera alors $(X \mid \Delta)=$ $(X \mid \Delta)^{d i v}$ pour préciser la catégorie de morphismes considérés.

2. Soit non-classiques. On notera alors $(X \mid \Delta)=(X \mid \Delta)^{Z}$ pour préciser la catégorie de morphismes considérés.

- Si les multiplicités sont rationnelles $($ ou $+\infty)$, les morphismes sont nécessairement non-classiques. On le précisera néammoins en notant : $(X \mid \Delta)=(X \mid \Delta)^{Q}$.

- On notera Georb ${ }^{*}, *=Q, Z$, div, l'une des trois catégories dont les objets sont les orbifoldes géométriques lisses, les morphismes étant de l'un des trois types: $Q, Z$ ou div. 
Definition 5.1 1. Une courbe rationnelle orbifolde lisse est une orbifolde géométrique $\left(\mathbb{P}^{1} \mid \Delta^{\prime}\right)$ telle que deg $\left(K_{\left(\mathbb{P}^{1} \mid \Delta^{\prime}\right)}\right)<0$.

2. Soit $(X \mid \Delta)$ une orbifolde géométrique lisse. Une courbe rationnelle orbifolde (ou $\Delta$-courbe rationnelle) $R \subset(X \mid \Delta)$ est l'image d'un morphisme orbifolde non-constant et birationnel sur son image $\nu:\left(\mathbb{P}^{1} \mid \Delta^{\prime}\right) \rightarrow(X \mid \Delta)$, dans lequel $\left(\mathbb{P}^{1} \mid \Delta^{\prime}\right)$ est une courbe orbifolde rationnelle lisse.

Il y a donc trois types de courbes orbifoldes, selon la catégorie (de morphismes) considérée :

1. Les $\Delta^{\text {div }}$-courbes rationnelles divisibles si $(X \mid \Delta)^{\text {div }}$ est entière et si $\nu$ est un morphisme orbifolde divisible. Leur ensemble est noté $\operatorname{Ratl}(X \mid \Delta)^{\text {div }}$.

2. Les $\Delta^{Z}$-courbes rationnelles entières si $(X \mid \Delta)^{\text {div }}$ est entière et si $\nu$ est un morphisme orbifolde non-classique. Leur ensemble est noté $\operatorname{Ratl}(X \mid \Delta)^{Z}$. On a donc : $\operatorname{Ratl}(X \mid \Delta)^{\operatorname{div}} \subset \operatorname{Ratl}(X \mid \Delta)^{Z}$ :

3. Les $\Delta^{Q}$-courbes rationnelles, en général, si $\nu$ est un morphisme orbifolde non-classique. Leur ensemble est noté $\operatorname{Ratl}(X \mid \Delta)^{Q}$.

Lorsque $(X \mid \Delta)$ est logarithmique (i.e : si $\Delta=\lceil\Delta\rceil$ ), ces trois notions coincident cependant.

Exemple 5.2 Si $X=\mathbb{P}_{1}$, et si $\Delta^{\prime}=\sum_{j}\left(1-\frac{1}{m_{j}}\right)$. $p_{j}$, avec $m_{j}>1, \forall j \in J$, les $p_{j}$ étant des points distincts de $X$, alors $\left(\mathbb{P}_{1} \mid \Delta\right)$ est rationnelle orbifolde si et seulement si $\sum_{j}\left(1-1 / m_{j}\right)<2$.

Lorsque $\Delta^{\prime}$ est entière, c'est le cas si et seulement si la suite ordonnée croissante des $m_{j}, j \in J$ est l'une des suivantes :

$|J| \leq 2$ : quelconque, $(+\infty,+\infty)$ exclue.

$|J|=3:(2,2, m), \forall m<+\infty ;(2,3,3),(2,3,4) ;(2,3,5)$.

De l'exemple 2.40, on déduit :

Proposition 5.3 Soit $(X \mid \Delta), \Delta:=\left(\sum_{j}\left(1-\frac{1}{m_{j}}\right) . D_{j}\right)$ une orbifolde géométrique lisse, et $R \subset X$ une courbe (rationnelle) irréductible non contenue dans le support de $\Delta$, de normalisation $\nu: \mathbb{P}^{1} \rightarrow X$. On a équivalence entre les deux propriétés suivantes:

1. $R \subset(X \mid \Delta)^{Q}$ est une $\Delta^{Q}$-courbe rationnelle.

2. $\operatorname{deg}\left(K_{\mathbb{P}^{1}}+\Delta^{\prime}\right)=-2+\sum_{p \in \mathbb{P}^{1}}\left(1-\frac{1}{\mu_{p}}\right)<0, \Delta^{\prime}:=\sum_{p \in \mathbb{P}^{1}}\left(1-\frac{1}{\mu_{p}}\right) \cdot\{p\}$ étant le plus petit diviseur orbifolde sur $\mathbb{P}^{1}$ qui fait de $\nu:\left(\mathbb{P}^{1} \mid \Delta^{\prime}\right) \rightarrow(X \mid \Delta)$ un morphisme orbifolde. Les $\mu_{p}$ sont définis comme suit.

Pour tous $j, p \in \mathbb{P}^{1}$, on définit $t_{j, p}$ par $: \nu^{*}\left(D_{j}\right)=\sum_{p \in \mathbb{P}^{1}} t_{j, p} .\{p\}$, et $\mu_{p}:=$ $\max _{j}\left\{1, m_{j} / t_{j, p}\right\}, j$ décrivant l'ensemble des indices tels que $: t_{j, p}>0$. 
Remarque 5.4 1. Si l'on veut que $R$ soit une $\Delta^{Z}$-courbe rationnelle, et si $(X \mid \Delta)$ est entière, on doit choisir : $\mu_{p}:=\max _{j}\left\{\left\lceil m_{j} / t_{j, p}\right\rceil\right\}$.

2. Si $\Delta$ est entière et finie, et si l'on veut que $R$ soit une $\Delta^{d i v}$-courbe rationnelle, on doit choisir : $\mu_{p}:=\operatorname{ppcm}_{j}\left\{m_{j, p}^{\prime}:=m_{j} / \operatorname{pgcd}\left\{t_{j, p}, m_{j}\right\}\right\}$.

3. Si $(X \mid \Delta)$ est entière, on a donc : $\operatorname{Ratl}(X \mid \Delta)^{Q} \supset \operatorname{Ratl}(X \mid \Delta)^{Z} \supset$ $\operatorname{Ratl}(X \mid \Delta)^{d i v}$. Ces inclusions sont strictes en général.

\section{Exemple 5.5}

1. Si $R$, rationnelle, a tous ses contacts avec chacun des $D_{j}$ d'ordre au moins $m_{j}$, alors $R$ est $\Delta^{Q}$-rationnelle, avec $: \Delta^{\prime}=0$.

2. Si $(X \mid \Delta)$ est une orbifolde géométrique lisse logarithmique $(\Delta=$ $\operatorname{supp}(\Delta))$, les $\Delta$-courbes rationnelles sont les courbes rationnelles $R$ de $X$ dont la normalisation rencontre $\Delta$ en, au plus, 1 point.

3. Considérons par exemple $X=\mathbb{P}^{2}$, et $\Delta$ la réunion de 4 droites en position générale, affectées de multiplicités $3,3,5,7$. Donc $\left(\mathbb{P}^{2} \mid \Delta\right)$ est Fano, puisque $1 / 3+1 / 3+1 / 5+1 / 7=106 / 105>1$. Il n'existe qu'un nombre fini de droites $\Delta^{Q}$-rationnelles, puisqu'une droite rencontrant $\operatorname{Supp}(\Delta)$ en au moins 3 points, y acquiert des multiplicités au moins $3,3,7$, et que $1 / 3+$ $1 / 3+1 / 7=17 / 21<1$. Par contre, $\left(\mathbb{P}^{2} \mid \Delta\right)$ a une famille à un paramètre de coniques irréductibles $\Delta^{Q}$-rationnelles : les coniques tangentes à chacune des 4 droites de $\operatorname{Supp}(\Delta)$. Elles acquièrent en effet en leurs points de contact des multiplicités rationnelles $3 / 2,3 / 2,5 / 2,7 / 2$. Puisque $4-(2 / 3+2 / 3+2 / 5+$ $2 / 7)=4-2(1 / 3+1 / 3+1 / 5+1 / 7)=4-2(1+1 / 105)=2-2 / 105<2$, ces coniques sont bien $\Delta^{Q}$-rationnelles.

Par contre, ces coniques ne sont pas $\Delta^{Z}$-rationnelles, car $\lceil 3 / 2\rceil,\lceil 3 / 2\rceil,\lceil 5 / 2\rceil,\lceil 7 / 2\rceil=2,2,3,4$, et donc telles que : $-2+1 / 2+$ $1 / 2+2 / 3+3 / 4=5 / 12>0$. Il semble possible d'avoir des familles à nombre positif de paramètres de $\Delta^{\text {div }}$-courbes rationnelles, mais seulement pour de grands degrés (voir l'exemple 5.55 ci-dessous).

4. Soit $X$ une variété projective lisse et torique. Et $D$ son diviseur anticanonique torique (à croisements normaux). On affecte chacune des composantes de $D$ d'une multiplicité finie, et on note $\Delta$ le diviseur orbifolde résultant. Si $R$ est une courbe rationnelle torique (adhérence de l'orbite d'un sous-groupe algébrique à un paramètre du tore agissant sur $X)$, non contenue dans $D$, alors $R$ rencontre $D$ en au plus 2 points en lesquels elle est unibranche. C'est donc une $\Delta^{\text {div }}$-courbe rationnelle . 


\section{2 "Descente" de courbes rationnelles.}

La définition des $\Delta^{*}$-courbes rationnelles $(*=Q, Z$ ou div), est compatible avec celle des courbes rationnelles usuelles et des revêtements ramifiés. En effet :

Théorème 5.6 Soient $(X \mid \Delta)$, lisse et entière. On suppose qu'il existe un morphisme fini $f: \bar{X} \rightarrow X$, avec $\bar{X}$ lisse (projectif), ramifiant au moins (resp. exactement) au-dessus de $\Delta^{17}$. Soit $\bar{R} \subset \bar{X}$ une courbe rationnelle, et $R:=f(\bar{R}) \subset X$. Alors $R$ est une $\Delta^{\text {div }}$-courbe rationnelle.

Si l'on a seulement $m_{j}^{\prime} \geq m_{j}, \forall j$, alors $R$ est une $\Delta^{Z}$-courbe rationnelle.

Démonstration : Nous ne montrerons que le cas divisible (le cas nonclassique est similaire, plus simple).

Lorsque la restriction $f: R^{\prime} \rightarrow R$ est birationnelle, la conclusion résulte de ce que la composée de deux morphismes orbifoldes est un morphisme orbifolde (notant que $f: \bar{X} \rightarrow(X \mid \Delta)$ est un morphisme orbifolde). Dans le cas général, composant les inclusions de $R, \bar{R}$ dans $X, \bar{X}$ avec les normalisations, nous pouvons supposer que $R, \bar{R}$ sont lisses rationnelles (pour simplifier les notations).

Soit maintenant $\bar{b} \in \bar{R}$ tel que $b:=f(b) \in D_{j}$. Soit $e(\bar{b})$ l'ordre de ramification de $f: \bar{R} \rightarrow R$ en $\bar{b}$, et $\bar{D}_{j}:=f^{-1}\left(D_{j}\right)$.

On note aussi $e(b):=\operatorname{pgcd}\left\{e(\bar{b}), \bar{b} \in f^{-1}(b) \cap \bar{R}\right\}$.

La formule de projection fournit pour les nombres d'intersection locaux près de $b, \bar{b}$ :

$$
m_{j}^{\prime} \cdot\left(\bar{D}_{j} \cdot \bar{R}\right)_{\bar{b}}=\left(f^{*}\left(D_{j}\right) \cdot \bar{R}\right)_{\bar{b}}=\left(D_{j} \cdot f_{*}(R)\right)_{b}=e(\bar{b}) \cdot\left(D_{j} \cdot R\right)_{b}
$$

D'où l'on déduit, par le théorème de Bezout, que, pour tout $j$ tel que $f(b) \in D_{j}, m_{j}^{\prime}$, et donc aussi $m_{j}$, divise $e(b) .\left(D_{j} . R\right)_{b}$.

Et donc, désignant par $\Delta^{\prime}$ le plus petit diviseur orbifolde entier sur $R$ faisant de l'inclusion (normalisée) de $R$ dans $X$ un morphisme $\Delta$-orbifolde divisible : $m_{\Delta^{\prime}}(b)$ divise $e(b)$. Donc : $K_{R^{\prime}} \geq f^{*}\left(K_{R}+\Delta^{\prime}\right)$, ce qui achève la démonstration du théorème.

\footnotetext{
${ }^{17}$ Ceci signifie que, si $\Delta:=\sum_{j}\left(1-1 / m_{j}\right) \cdot D_{j}$, alors $f$ est étale au-dessus du complémentaire du support de $\Delta$, et ramifie à l'ordre $m_{j}^{\prime}$ multiple de $m_{j}$ (resp. $m_{j}^{\prime}=$ $\left.m_{j}, \forall j\right)$ en chaque point $\bar{x}$ de $\bar{X}$ tel que $f(\bar{x}) \in D_{j}$.
} 
Le théorème résulte aussi du lemme suivant, qui en donne une version quantitative, appliqué à $R^{\prime}:=\bar{R}$ et $R=R$, puisque $g(\bar{R})=g(R)=0$ :

Lemme 5.7 Soit $f: R^{\prime} \rightarrow R$ un morphisme surjectif de degré $d>0$ entre courbes projectives lisses et connexes. Pour tout $b^{\prime} \in R^{\prime}$, on note e $\left(b^{\prime}\right)$ l'ordre de ramification de $f$ en $b^{\prime}$, et pour $b \in R$, par $e(b):=\operatorname{pgcd}\left\{e\left(b^{\prime}\right), b^{\prime} \in f^{-1}(b)\right\}$, et enfin par $e^{+}(b):=\max \left\{e\left(b^{\prime}\right), b^{\prime} \in f^{-1}(b)\right\} \geq e(b)$.

$$
\text { Alors : } \frac{2\left(g\left(R^{\prime}\right)-1\right)}{d} \geq \frac{2(g(R)-1)}{d}+\sum_{b}\left(1-\frac{1}{e^{+}(b)}\right) \geq \frac{2(g(R)-1)}{d}+\sum_{b}\left(1-\frac{1}{e(b)}\right) .
$$

Démonstration : Appliquant la formule de Riemann-Hurwitz à $f: R^{\prime} \rightarrow$ $R$, on obtient :

$2 g\left(R^{\prime}\right)-2=2 d \cdot(g(R)-1)+\sum_{b}\left(\sum_{\bar{b} \in f^{-1}(b)}(e(\bar{b})-1)\right)=2 d \cdot(g(R)-1)+\sum_{b}\left(d-\sum_{b^{\prime} \in f^{-1}(b)} 1\right)$

Divisant par $d$, nous obtenons :

$$
\frac{2\left(g\left(R^{\prime}\right)-1\right)}{d}=\frac{2(g(R)-1)}{d}+\sum_{b}\left(1-\frac{\sum_{b^{\prime}} 1}{\sum_{b^{\prime}} e\left(b^{\prime}\right)}\right)
$$

La conclusion résulte alors de ce que $: \frac{\sum_{b^{\prime}} 1}{\sum_{b^{\prime}} e\left(b^{\prime}\right)} \leq e(b) \leq e^{+}(b)$

\subsection{Lemme de "cassage" orbifolde.}

Proposition 5.8 Soit $(X \mid \Delta)$ une orbifolde lisse avec $X$ projective connexe, et $C_{t}$ une famille algébrique couvrante de courbes $\Delta^{Q}$-rationnelles. Alors : $\left(K_{X}+\Delta\right) \cdot C_{t}<0$.

Démonstration : Elle se fait en passant au graphe d'incidence normalisé $q: Z \rightarrow X$ de la famille, et en utilisant le fait que $q^{*}\left(K_{X}\right) \leq K_{Z}$, puisque la famille est couvrante. On conclut avec la formule d'adjonction : $\left(K_{X}+\right.$ $\Delta) \cdot C_{t}=q^{*}\left(K_{X}+\Delta\right) \cdot C_{t} \leq\left(K_{Z}+q^{*}(\Delta)\right) \mid C_{t}=\operatorname{deg}\left(K_{C_{t}}+q^{*}(\Delta)\right)<0$, puisque $C_{t}$ est $\Delta$-rationnelle. (On a identifié $C_{t}$ et son image dans $Z$ )

La question centrale d'existence de la géométrie des $\Delta$-courbes rationnelles est la réciproque : 
Question 5.9 Soit $(X \mid \Delta)$ une orbifolde lisse avec $X$ projective connexe. Soit $C \subset X$ une courbe projective irréductible non contenue dans le support de $\Delta$. Soit $a \in C, a \notin S u p p(\Delta)$. Si $\left(K_{X}+\Delta\right) . C<0$, existe-t'il une $\Delta$-courbe rationnelle passant par a?

Nous allons déduire du classique "lemme de cassage" ("Bend and break") de Miyaoka-Mori [Mi-Mo 86] une réponse positive dans le cas (très) particulier suivant.

Théorème 5.10 Soit $(X \mid \Delta)$ une orbifolde géométrique lisse, entière et à multiplicités finies. On suppose $X$ projective. On suppose aussi qu'il existe un morphisme fini $f: \bar{X} \rightarrow X$, avec $\bar{X}$ lisse, ramifiant au moins (au sens divisible) au-dessus de $\Delta^{18}$.

Soit $C \subset X$ une courbe projective irréductible, non contenue dans $\operatorname{Supp}(\Delta)$, et telle que $\left(K_{X}+\Delta\right) . C<0$.

Alors : si $a \in C$ n'appartient pas au support de $\Delta$, il existe une $\Delta^{\text {div }}$ courbe rationnelle $R$ de $X$ passant par $a$.

Si $H$ est un diviseur ample sur $X$, on peut choisir $R$ telle que :

$$
H . R \leq \frac{2 . H . C}{-\left(K_{X}+\Delta\right) \cdot C}
$$

Remarque 5.11 L'hypothèse très restrictive de l'existence de $f: \bar{X} \rightarrow X$ devrait pouvoir être supprimée en remplaçant ce revêtement par le champ algébrique (de Deligne-Mumford) associé à $(X \mid \Delta)$, et en établissant un "lemme de cassage" dans cette catégorie.

Démonstration : Soit $\bar{C}$ une composante irréductible de $f^{-1}(C)$. Alors $K_{\bar{X}} \cdot \bar{C}=t . f^{*}\left(K_{X}+\Delta\right) . C<0$ si $f_{*}(\bar{C})=t . C$. Soit $\bar{H}:=f^{*}(H)$. Si $\bar{a} \in \bar{C}$ est au-dessus de $a$, il existe, d'après [Mi-Mo 86], une courbe rationnelle $\bar{R}$ passant par $\bar{a}$ et telle que :

$$
\bar{H} \cdot \bar{R} \leq 2 \cdot \frac{\bar{H} \cdot \bar{C}}{-\left(K_{\bar{X}} \cdot \bar{C}\right)}
$$

Posant $d . R:=f_{*}(\bar{R})$, avec $R:=f(\bar{R})$. Il suffit donc de montrer que $R$ est une $\Delta$-courbe rationnelle. Le théorème 5.6 ci-dessus achève donc la démonstration

\footnotetext{
${ }^{18}$ voir 5.6 ci-dessus.
} 
Corollaire 5.12 Soit $(X \mid \Delta)$ et $f: \bar{X} \rightarrow X$ satisfaisant les hypothèses du théorème précédent. On suppose que $\left(K_{X}+\Delta\right) . C_{t}<0$ pour une famille $C_{t}$ de courbes irréductibles de $X$ recouvrant un ouvert (analytique) non vide de $X$. Alors un ouvert de Zariski non-vide de $X$ est recouvert par une famille algébrique de courbes $\Delta$-rationnelles $R_{s}$.

Si la famille $C_{t}$ est algébrique, le $H$-degré des $R_{s}$ satisfait la borne de 5.10 .

\subsection{Semi-positivité générique.}

La présente section est suggérée par une question orale d'Amaël Broustet ([Br 08$])$ : "peut-t-on étendre aux orbifoldes géométriques le théorème de semi-positivité géométrique de Miyaoka (voir [Miy 87] et [SP 92]) ?"

Plus précisément :

Question 5.13 Soit $(X \mid \Delta)$ une orbifolde lisse et finie, avec $X \in \mathcal{C}$. S'il existe des entiers strictement positifs $m, p$ tels que $S^{m} \Omega^{p}(X \mid \Delta)$ ait un quotient $\mathcal{Q}$ tel que $c_{1}(\mathcal{Q})$ ne soit pas pseudo-effectif, alors $(X \mid \Delta)$ est-elle uniréglée (i.e : génériquement recouverte par une famille de $\Delta$-courbes rationnelles)?

On a une réponse positive lorsque $\Delta=0$ si $X$ est projective ([C-Pe 06]), et aussi dans le cas (très) particulier suivant :

Théorème 5.14 Soit $(X \mid \Delta)$ une orbifolde géométrique lisse, entière et à multiplicités finies. On suppose $X$ projective. On suppose aussi qu'il existe un morphisme fini $f: \bar{X} \rightarrow X$, avec $Y$ lisse, ramifiant exactement (au sens divisible) au-dessus de $\Delta^{19}$.

Alors $(X \mid \Delta)$ est uniréglée s'il existe des entiers strictement positifs $m, p$ tels que $S^{m} \Omega^{p}(X \mid \Delta)$ ait un quotient $\mathcal{Q}$ tel que $c_{1}(\mathcal{Q})$ ne soit pas pseudoeffectif, et si $m$ est divisible par toutes les multiplicités de $\Delta$.

Démonstration : C'est une réduction immédiate au cas où $\Delta=0$. En effet : $f^{*}\left(S^{m} \Omega^{p}(X \mid \Delta)\right)=\operatorname{Sym}^{m}\left(\Omega_{\bar{X}}^{p}\right)$ a pour quotient $f^{*}(\mathcal{Q})$, qui n'est pas pseudo-effectif. Donc $\bar{X}$ est uniréglée, par [C-Pe 06], theorem 0.3, et donc aussi $(X \mid \Delta)$, par 5.6

\footnotetext{
${ }^{19} \mathrm{C}$ 'est donc un quotient global au sens de 5.15 ci-dessous.
} 


\subsection{Quotients globaux et relèvement de courbes ra- tionnelles orbifoldes.}

Definition 5.15 Soit $(X \mid \Delta)$ une orbifolde géométrique lisse, entière et finie (avec $X \in \mathcal{C}$ ). On dit que $(X \mid \Delta)$ est un quotient global s'il existe $f: X^{\prime} \rightarrow$ $X$ finie, holomorphe, avec $X^{\prime}$ lisse, et ramifiant exactement au-dessus de $\Delta$ au sens de 5.6.

On dit alors que $f: X^{\prime} \rightarrow(X \mid \Delta)$ est galoisien (de groupe $G$ ) s'il existe un groupe fini $G$ agissant effectivement sur $X^{\prime}$ tel que $f$ soit le quotient $X^{\prime} \rightarrow X=X^{\prime} / G$.

Tout quotient global est aussi un quotient global galoisien (par la construction usuelle, en remarquant qu'elle préserve ici la lissité des revêtements $X^{\prime}$ ).

Le résultat suivant permet de ramener les assertions sur les courbes rationnelles orbifoldes au cas non-orbifolde pour les quotients globaux.

Théorème 5.16 Soit $f: X^{\prime} \rightarrow(X \mid \Delta)$ un quotient global, et $R \subset X$ une $\Delta^{\text {div }}$-courbe rationnelle. Alors toute composante irréductible $R^{\prime} \subset X^{\prime}$ de $f^{-1}(R)$ est une courbe rationnelle.

Démonstration : Considérons le diagramme commutatif :

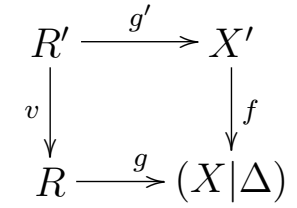

dans lequel les flèches horizontales sont les normalisations composées avec les inclusions, tandis que $v$ est génériquement la restriction de $f$.

Soit $b^{\prime} \in R^{\prime}, b:=v\left(b^{\prime}\right)$, et $t_{j}, t_{j}^{\prime}$ respectivement les ordres de contacts en $b$ et $b^{\prime}$ respectivement de $D_{j}$ avec $R$, et de $f^{-1}\left(D_{j}\right)$ avec $R^{\prime}$. Notons enfin $e\left(b^{\prime}\right):=e$ l'ordre de ramification en $b^{\prime}$ de $v$. La formule de projection (ou la commutativité du diagramme précédent) montre que :

$$
f_{*}\left(R^{\prime}\right) \cdot D_{j}=e \cdot g^{*}\left(D_{j}\right)=e \cdot t_{j}=R^{\prime} \cdot f^{*}\left(D_{j}\right)=m_{j} \cdot t_{j}^{\prime}
$$

Puisque $X^{\prime}$ est supposé lisse, $t_{j}^{\prime}$ est entier.

Notons $d_{j}:=\operatorname{pgcd}\left(t_{j}, m_{j}\right), u_{j}:=t_{j} / d_{j}, m_{j}^{\prime}:=m_{j} / d_{j}$.

Donc $\operatorname{pgcd}\left(u_{j}, m_{j}^{\prime}\right)=1$. 
On déduit donc de l'égalité ci-dessus que, pour tout $j \in J(b)$, ensemble des $j$ tels que $g(b) \in D_{j}$ :

$$
e . u_{j}=m_{j}^{\prime} \cdot t_{j}^{\prime}
$$

De sorte que : $u_{j}$ divise $t_{j}^{\prime}$ et $m_{j}^{\prime}$ divise $e$ pour tout $j \in J(b)$.

Soit alors : $m^{\prime}:=\operatorname{ppcm}\left\{m_{j}^{\prime}, j \in J(b)\right\}$.

Alors $m^{\prime}$ divise $e$ par ce qui précède. Posons : $e:=e^{\prime} \cdot m^{\prime}$.

L'assertion du théorème sera établie si l'on montre que $v: R^{\prime} \rightarrow\left(R \mid \Delta_{R}\right)$ est étale (ie : si $e=m^{\prime}$, ce qui équivaut ici à : $v^{*}\left(K_{R}+\Delta_{R}\right)=K_{R^{\prime}}, \Delta_{R}$ étant le diviseur orbifolde sur $R$ attribuant à (tout) $b \in R$ la multiplicité $m^{\prime}$ précédente, qui est la plus petite faisant de $g:\left(R \mid \Delta_{R}\right) \rightarrow(X \mid \Delta)$ un morphisme orbifolde divisible).

Nous allons montrer que $e^{\prime}=1$, ce qui établira donc le théorème.

Dans l'égalité suivante, les trois termes sont des entiers, et $e^{\prime}$ divise donc $e^{\prime \prime}:=\operatorname{pgcd}\left(e, t_{j}^{\prime}, j \in J(b)\right):$

$$
e^{\prime} \cdot\left(\frac{m^{\prime}}{m_{j}^{\prime}}\right)=\left(\frac{t_{j}^{\prime}}{u_{j}}\right), \forall j \in J(b)
$$

Dans des coordonnées locales adaptées, nous pouvons donc supposer (par lissité et revêtement étale local de $X^{\prime}$ ) que, si $s \in \mathbb{D}$ est une coordonnée locale sur $R^{\prime}$, alors :

$$
g^{\prime}(s)=\left(s^{t_{1}^{\prime}} \cdot\left(1+s \cdot w_{1}(s)\right), \ldots, s^{t_{n}^{\prime}} \cdot\left(1+s \cdot w_{n}(s)\right),\right.
$$

tandis que :

$$
f \circ g^{\prime}(s)=p_{1}\left(s^{e}\right), \ldots, p_{n}\left(s^{e}\right),
$$

les $w_{k}, p_{k}$ et $x_{k}$ ci-dessous étant des fonctions analytiques au voisinage de 0 .

On a donc, sur un voisinage de $0 \in \mathbb{D}$, disque unité de $\mathbb{C}$ :

$$
p_{k}\left(s^{e}\right)=s^{t^{\prime} \cdot m_{k}} \cdot\left(1+s . w_{k}(s)\right), \forall k:=1, \ldots, n .
$$

On en déduit que $e \cdot t_{k}=t_{k}^{\prime} \cdot m_{k}$ et que $\left(1+s \cdot w_{k}(s)\right)=1+s^{e} \cdot x_{k}\left(s^{e}\right)$, pour $s$ assez voisin de $0 \in \mathbb{C}$, et $k=1, \ldots, n$. Donc :

$$
g^{\prime}(s)=\left(s^{t^{\prime}} \cdot\left(1+s^{e} \cdot x_{1}\left(s^{e}\right)\right), \ldots, s^{t_{n}^{\prime}} \cdot\left(1+s^{e} \cdot x_{n}\left(s^{e}\right)\right) .\right.
$$

Puisque $g^{\prime}$ est génériquement injective près de 0 , on a bien :

$$
\operatorname{pgcd}\left(e, t_{k}^{\prime}, k=1, \ldots, n\right)=1 \text {. }
$$

Ce qui achève la preuve 
Remarque 5.17 Sous les hypothèses précédentes, la démonstration montre, plus généralement, que si $\left(R \mid \Delta_{R}\right) \rightarrow(X \mid \Delta)$ est un morphisme orbifolde, avec $R$ une courbe irréductible, et si $R^{\prime} \subset f^{-1}(R) \subset X^{\prime}$ est une composante irréductible, alors $v: R^{\prime} \rightarrow(R \mid \Delta)$ est étale (au sens orbifolde suivant :)

Definition 5.18 Soit $f:\left(B^{\prime} \mid \Delta_{B^{\prime}}\right) \rightarrow\left(B \mid \Delta_{B}\right)$ un morphisme fini surjectif entre courbes lisses projectives et connexes $B^{\prime}, B$. On dit que $f$ est étale si $f^{*}\left(K_{B}+\Delta_{B}\right)=K_{B^{\prime}}+\Delta_{B^{\prime}}$. Ceci équivaut à $: e\left(b^{\prime}\right) \cdot m^{\prime}\left(b^{\prime}\right)=m(b)$ pour tout $b^{\prime} \in B^{\prime}, e\left(b^{\prime}\right)$ (resp. $m\left(b^{\prime}\right)$, resp. $m(b)$ ) désignant l'ordre de ramification en $b^{\prime}$ (resp. la multiplicité de $\Delta_{B^{\prime}}$ en $b^{\prime}$, resp. la multiplicité de $\Delta_{B}$ en $b:=f\left(b^{\prime}\right)$ ).

Si $f:\left(B^{\prime} \mid \Delta_{B^{\prime}}\right) \rightarrow\left(B \mid \Delta_{B}\right)$ est étale, alors $\left(B^{\prime} \mid \Delta_{B^{\prime}}\right)$ est rationnelle (resp. elliptique) si et seulement si $\left(B \mid \Delta_{B}\right)$ l'est. (On dit que $\left(B^{\prime} \mid \Delta_{B^{\prime}}\right)$ est elliptique si $\left.\operatorname{deg}\left(K_{\left(B^{\prime} \mid \Delta_{B^{\prime}}\right)}\right)=0\right)$.

\subsection{Uniréglage, Connexité Rationnelle orbifolde}

Definition 5.19 Soit $(X \mid \Delta)$ une orbifolde géométrique lisse, avec $X \in \mathcal{C}$ connexe. On dit que $(X \mid \Delta)$ est :

1. uniréglée (abrégé en : UR) si le point générique de $X$ est contenu dans une courbe rationnelle orbifolde de $(X \mid \Delta)$.

2. rationnellement engendrée (en abrégé : RE) si, pour toute fibration méromorphe dominante $f:(X \mid \Delta) \rightarrow Y$, toute base orbifolde stable $\left[Y \mid \Delta(f, \Delta)^{Q}\right]$ de $f$ est uniréglée. (Cette notion est introduite, en l'absence de structures orbifoldes, dans [Ca95]).

Puisque les invariances birationnelles, de l'uniréglage et des bases orbifoldes stables, sont inconnues, nous supposons l'uniréglage de toute base orbifolde stable.

Il est essentiel que les bases orbifoldes soient considérées dans la catégorie $G_{\text {eorb }}^{Q}$ à cause des exemples 5.36 et 5.43 ci-dessous.

3. faiblement rationnellement connexe par chaines (en abrégé : $F R C C)$ si tout couple de points génériques de $X$ est contenu dans une chaine (ie : une réunion finie connexe) de courbes rationnelles orbifoldes de $(X \mid \Delta)$, ou de leurs limites (considérées dans Chow $(X))$.

4. rationnellement connexe par chaines (en abrégé : RCC) si tout couple de points génériques de $X$ est contenu dans une chaine de courbes rationnelles orbifoldes de $(X \mid \Delta)$.

5. rationnellement connexe (en abrégé : $R C$ ) si tout couple de points génériques de $X$ est contenu dans une courbe rationnelle orbifolde de $(X \mid \Delta)$. 
6. fortement rationnellement connexe (en abrégé : $R C^{+}$) si tout ensemble fini de points génériques de $X$ est contenu dans une courbe rationnelle orbifolde de $(X \mid \Delta)$

7. Les trois notions de $\Delta$-courbes rationnelles (divisible, entière ou non) peuvent être employées ci-dessus, et conduisent d'ailleurs peut-être à des notions équivalentes dans nombre de cas importants (mais pas toujours : si $X=\mathbb{P}_{1}$, par exemple, les notions $U R^{Q}$ et $U R^{Z}$ diffèrent si $\Delta$ est à multiplicités rationnelles et si son support a au moins 4 points).

Si l'on veut préciser la notion de courbe rationnelle orbifolde employée, on utilisera la notation $U R^{d i v}, R E^{d i v}, R C C^{d i v}, F R C C^{d i v}, R C^{d i v}, R C^{+d i v}$, si l'on est dans la catégorie Georb ${ }^{\text {div }}$, avec des notations similaires pour Georb*, $*=Q, Z$.

8. Les notions plus fortes suivantes semblent devoir être utiles dans l'etude de l'invariance birationnelle des notions précédentes (voir le lemme 5.31 cidessous).

Nous dirons que $(X \mid \Delta)$ est précisément uniréglée (noté $U R_{\text {evit }}$ ) si pour tout sous ensemble analytique $Z$ de $X$ de codimension au moins 2 , et tout point générique de $X$, il existe une courbe rationnelle de $(X \mid \Delta)$ passant par ce point et évitant (ie : ne rencontrant pas) $Z$. On a donc les trois notions : $U R_{\text {evit }}^{*}$, avec $*=Z, Q$, div.

On définit de la même façon les notions $R E_{\text {evit }}, F R C C_{\text {evit }}, R C C_{\text {evit }}$, etc...

On a, bien sûr, l'implication : $U R_{\text {evit }}^{*} \Longrightarrow U R^{*}, \ldots, R C_{\text {evit }}^{*} \Longrightarrow R C^{*}$ et la démonstration du lemme 5.31 montre que les propriétés $U R_{\text {evit }}, \ldots, R C_{\text {evitt }}^{+}$ sont des invariants biméromorphes.

\section{Exemple 5.20}

1. Si $(X \mid \Delta)$ est lisse, Fano (ie : $-\left(K_{X}+\Delta\right)$ est ample sur $\left.X\right)$, et s'il existe $f: \bar{X} \rightarrow X$ satisfaisant les hypothèses du théorème 5.10, alors $(X \mid \Delta)$ est uniréglée.

2. Soit $(X \mid \Delta)$ l'exemple torique de 5.5 .4 ci-dessus. Alors $(X \mid \Delta)$ est rationnellement connexe, puisque toutes ses courbes toriques rationnelles noncontenues dans $D$ sont $\Delta$-rationnelles.

3. L'orbifolde $\left(\mathbb{P}_{2} \mid \Delta\right)$ de l'exemple 5.5 .3 ci-dessus est $R C$ (mais avec des coniques $\Delta$-rationnelles non-entières). L'argument de comptage de l'exemple 5.55 semble indiquer qu'elle devrait être $\Delta$-rationnelle pour des courbes rationnelles de degré assez grand sans structure orbifolde.

\section{Remarque 5.21}


- On a les implications évidentes :

$R C^{+} \Longrightarrow R C \Longrightarrow R C C \Longrightarrow F R C C \Longrightarrow$ Uniréglée.

$R E \Longrightarrow$ Uniréglée.

Par contre, l'implication : $F R C C \Longrightarrow R E$ (ou même : $R C^{+} \Longrightarrow R E$ ) n'est évidente que si l'on suppose que la propriété $F R C C$ (resp. $R C^{+}$) est biméromorphiquement invariante (dans le cadre orbifolde et si les multiplicités sont finies, du moins. Voir la remarque 5.24.1 ci-dessous).

- On a aussi (à l'aide de l'invariance biméromorphe) les implications évidentes :

$R C_{\text {evit }}^{+} \Longrightarrow R C_{\text {evit }} \Longrightarrow R C C_{\text {evit }} \Longrightarrow F R C C_{\text {evit }} \Longrightarrow R E_{\text {evit }} \Longrightarrow U R_{\text {evit }}$

- Lorsque $\Delta=0$, et si $X$ est projective lisse, on a évidemment : $R C C \Longleftrightarrow$ $F R C C$, ainsi que les difficiles implications réciproques, basées sur le "lemme de collage" 20 :

- $R E \Longrightarrow R C C=F R C C([\mathrm{GHS} 03])$ et :

- $R C C \Longrightarrow R C \Longrightarrow R C^{+}$([KoMiMo92]).

\section{Question 5.22}

1. Les propriétés FRCC et RCC sont-elles toujours équivalentes? (ie: $F R C C \Longrightarrow R C C$ ?)

2. Les propriétés précédentes (définies en 5.19) sont-elles stables par déformation (au sens de 12.1)?

3. Les résultats de [KoMiMo92] et [GHS01] subsistent-ils dans le cadre orbifolde géométrique? Plus précisément : soit $(X \mid \Delta)$ une orbifolde géométrique lisse, avec $X \in \mathcal{C}$, connexe. Alors :

4. Si $(X \mid \Delta)$ est RCC, est-elle RC? Plus généralement, tout sousensemble fini de $X$ est-il alors contenu dans une courbe rationnelle orbifolde (irréductible) de $(X \mid \Delta)$ ?

5. Si $f:(X \mid \Delta)^{\text {div }} \rightarrow B$ est une fibration sur une courbe projective, et si les fibres orbifoldes génériques de $f$ sont $R C$, alors $f$ admet-t-elle une multisection orbifolde (ie : une multisection $s: B \rightarrow X$ d'image normalisée irréductible $B^{\prime}$ telle que $f:\left(B^{\prime} \mid \Delta_{B^{\prime}}\right) \rightarrow(B \mid \Delta(f, \Delta))$ soit étale au sens orbifolde, si $g:\left(B^{\prime} \mid \Delta_{B^{\prime}}\right) \rightarrow(X \mid \Delta)$ est un morphisme orbifolde divisible, $\Delta_{B^{\prime}}$ étant la structure orbifolde minimale sur $B^{\prime}$ avec cette propriété. Voir 5.37 pour plus de détails pour une formulation précise.)?

6. Nous donnerons ci-dessous au $\S 5.8$ une réponse affirmative aux questions posées pour les quotients globaux et les courbes $\Delta^{\text {div }}$-rationnelles.

20 "Glueing lemma" dans l'original [Ko-Mi-Mo]. 
Proposition 5.23 Soit $f:(X \mid \Delta) \rightarrow Y$ une fibration méromorphe dominante, avec $X \in \mathcal{C}$ connexe, et $(X \mid \Delta)$ lisse. Si la fibre orbifolde (générique) $\left(X_{y} \mid \Delta_{y}\right)$ et la base orbifolde stable de $f$ sont $R E$, alors $(X \mid \Delta)$ est $R E$.

Démonstration : Soit $g:(X \mid \Delta) \rightarrow Z$ une fibration méromorphe dominante. Si $g$ ne se factorise pas par $f$, la base orbifolde stable de $g$ est uniréglée, puisque recouverte par les images par $g$ des fibres orbifoldes de $f$, qui sont RE, et dont les images (munies de leur structures orbifoldes stables) par $g$ sont donc uniréglées. Sinon, $g=h \circ f$, et $h:[(Y \mid \Delta(f, \Delta)] \rightarrow Z$ a pour base orbifolde stable $\left[\left(Z \mid \Delta_{Z}\right)\right]$ celle de $h$ (par la propriété 3.13). Donc $\left[\left(Z \mid \Delta_{Z}\right)\right]$ est uniréglée, puisque $\left[\left(Y \mid \Delta_{Y}\right)\right]$ est RE, par hypothèse.

\section{Remarque 5.24}

1. La propriété $R C C$ n'est pas préservée par équivalence biméromorphe (avec les définitions ci-dessus) pour les orbifoldes géométriques logarithmiques. Par exemple : $\left(\mathbb{P}^{2} \mid D\right)$ est (Fano et) $R C C$, si $D$ est la réunion de 2 droites distinctes concourantes en 1 point $a$, puisque les droites passant par $a$ sont $D$-rationnelles (ce sont en fait les seules $D$-courbes rationnelles). Par éclatement de $a$, on obtient une orbifolde géométrique qui n'est pas $R C C$ : elle admet une fibration orbifolde évidente sur l' orbifolde géométrique $\left(\mathbb{P}^{1} \mid D^{\prime}\right)$, où $D^{\prime}$ est la réunion (réduite) de 2 points. Donc $\kappa\left(\mathbb{P}^{1} \mid D^{\prime}\right)=0$.

En particulier, l'orbifolde logarithmique $\left(\mathbb{P}_{2} \mid D\right)$ est Fano, sans être RE.

2. Le phénomène précédent semble lié au fait que les singularités du couple $\left(\mathbb{P}^{2}, D\right)$ sont log-canoniques $(l c)$, mais pas $k l t$. D'où la :

3. Question : Pour les orbifoldes géométriques lisses "finies", les propriétés $R C$ et $R C C$ sont-elles stables par équivalence biméromorphe? Les propriétés $R C$ et $R C_{\text {evit }}$ sont-t'elles équivalentes? Même question pour les autres propriétés : $U R, R E, F R C C, \ldots$ ?

\subsection{Quotients rationnels orbifoldes}

Théorème 5.25 Soit $(X \mid \Delta)$ une orbifolde géométrique lisse, avec $X \in \mathcal{C}$ connexe. Il existe une unique fibration méromorphe dominante $R:=R_{(X \mid \Delta)}$ : $X \rightarrow R(X \mid \Delta)$ telle que $e^{21}:$

1. Les fibres orbifoldes de $R$ sont $F R C C$.

2. Si $y \in R(X \mid \Delta)$ est général, la fibre $X_{y}$ de $R$ au-dessus de y ne rencontre aucune courbe rationnelle orbifolde de $(X \mid \Delta)$.

\footnotetext{
${ }^{21}$ Ici encore, le résultat est valable pour les trois notions de $\Delta$-courbe rationnelle.
} 
Démonstration : Nous utilisons ici (brièvement) l'appendice technique 5.12 ci-dessous.

Si $(X \mid \Delta)$ n'est pas uniréglée, $R=i d_{X}$. Sinon, on désigne par $A \subset \mathcal{C}(X)$ l'ensemble des points paramétrant une courbe rationnelle orbifolde (réduite) de $(X \mid \Delta)$. On va montrer que $A$ est Z-régulier (au sens de 5.57). La conclusion résultera alors de 5.58. Soit donc $B \subset \mathcal{C}(X)$ un sous-ensemble analytique fermé irréductible (donc compact, par [Lieb78]) tel que $Z_{a}$ soit une courbe rationnelle orbifolde réduite de $(X \mid \Delta)$ pour un sous-ensemble $A^{\prime}:=A \cap B$ qui n'est pas contenu dans une réunion dénombrable de sous-ensembles analytiques fermés stricts de $B$. Le membre générique $Z_{b}$ de la famille paramétrée par $B$ est donc une courbe rationnelle de $X$. Soit $Z \subset B \times X$ le graphe d'incidence (normalisé) de la famille $Z_{b}, b \in B$, muni de ses projections $p: Z \rightarrow X$ et $q: Z \rightarrow B$. Au-dessus d'un ouvert de Zariski dense $B^{*}$ de $B, q$ est une submersion de fibre $\mathbb{P}_{1}$, et il existe une structure orbifolde fixe $\Delta_{P}$ sur $\mathbb{P}_{1}$ (à automorphisme près de $\left.\mathbb{P}_{1}\right)$ telle que $q_{a}:\left(Z_{a} \mid \Delta_{P}\right) \rightarrow(X \mid \Delta)$ soit un morphisme orbifolde, pour tout $a \in A$ " $\subset A^{\prime}, A$ " n'étant pas contenu dans une réunion dénombrable de sous-ensembles analytiques fermés stricts de $B$. Le morphisme $q_{a}$ est donc naturellement la restriction d'un morphisme orbifolde $q:\left(Z \mid \Delta_{Z}\right) \rightarrow(X \mid \Delta)$, pour une structure orbifolde $\Delta_{Z}$ sur $Z$ dont la restriction à $Z_{b}$ est une courbe rationnelle orbifolde, pour $b \in B$ générique.

Corollaire 5.26 Soit $(X \mid \Delta)$ une orbifolde géométrique lisse, avec $X \in \mathcal{C}$ connexe. Il existe $e^{22}$ une unique fibration méromorphe dominante $R^{*}:=$ $R_{(X \mid \Delta)}^{*}: X \rightarrow R^{*}(X \mid \Delta)$ telle que :

1. Les fibres orbifoldes de $R$ sont $R E$.

2. L'une des bases orbifoldes stables de $R^{*} n^{\prime e s t}$ pas uniréglée.

Démonstration : Si une base orbifolde $\left(X_{1} \mid \Delta_{1}\right):=[R(X \mid \Delta) \mid \Delta(R, \Delta)]$ n'est pas uniréglée au sens orbifolde, on prend $R=R^{*}$. Sinon, on considère $R_{1}:=R_{\left(X_{1} \mid \Delta_{1}\right)}$, et on itère la construction, raisonnant par récurrence sur $\operatorname{dim}(X)$. Les fibres orbifoldes de la fibration composées sont bien RE, par 5.23 .

Remarque 5.27 Si l'implication $R E \Longrightarrow R C C$ est vraie, alors $R=R^{*}$. Ce serait le cas si les questions 5.22 avaient une réponse positive.

\footnotetext{
${ }^{22}$ Le résultat est valable pour les trois notions de $\Delta$-courbe rationnelle.
} 


\subsection{Le cas des quotients globaux.}

Nous réduisons ici, grâce au théorème 5.16, l'étude des courbes $\Delta^{d i v}$ rationnelles à celle des courbes rationnelles usuelles lorsque $(X \mid \Delta)$ est un quotient global au sens de 5.15 .

Théorème 5.28 Soit $f: X^{\prime} \rightarrow(X \mid \Delta)$ un quotient global au sens de la définition 5.15. Alors :

1. $(X \mid \Delta)^{\text {div }}$ est uniréglée si et seulement si $X^{\prime}$ est uniréglée.

2. $(X \mid \Delta)^{\text {div }}$ est FRCC si et seulement si $X^{\prime}$ est $R C C$.

3. $(X \mid \Delta)^{\text {div }}$ est $R C$ si et seulement si $X^{\prime}$ est $R C$.

4. $(X \mid \Delta)^{\text {div }}$ est $R C^{+}$si et seulement si $X^{\prime}$ est $R C^{+}$.

Démonstration : Elle est immédiate, d'après les définitions, et les théorèmes 5.6 et 5.16 .

Corollaire 5.29 Soit $f: X^{\prime} \rightarrow(X \mid \Delta)$ un quotient global au sens de la définition 5.15. Alors les propriétés suivantes sont équivalentes :

1. $(X \mid \Delta)^{\text {div }}$ est $F R C C$

2. $(X \mid \Delta)^{\text {div }}$ est $R C C$

3. $(X \mid \Delta)^{\text {div }}$ est $R C$

4. $(X \mid \Delta)^{\text {div }}$ est $R C^{+}$

Démonstration : Evidente d'après 5.28 et l'équivalence entre les propriétés $R C^{+}$et $F R C C$ pour $X^{\prime}$ (qui résulte de [Ko-Mi-Mo92]).

Nous établissons maintenant l'équivalence biméromorphe (qui n'est pas aussi évidente que lorsque $\Delta=0$ ) :

Corollaire 5.30 Soit $f: X^{\prime} \rightarrow(X \mid \Delta)$ un quotient global au sens de la définition 5.15. Si $\left(X_{1} \mid \Delta^{\prime}\right)$ est une orbifolde lisse birationnellement équivalente à $(X \mid \Delta)$, alors :

1. $(X \mid \Delta)^{\text {div }}$ est uniréglée si et seulement si $\left(X_{1} \mid \Delta_{1}\right)^{\text {div }}$ est uniréglée.

2. $(X \mid \Delta)^{\text {div }}$ est $R C$ si et seulement si $\left(X_{1} \mid \Delta_{1}\right)^{\text {div }}$ est $R C$.

Démonstration : Il suffit d'établir les assertions lorsqu'il existe une application biméromorphe $g: X_{1} \rightarrow X$ qui induit une équivalence biméromorphe élémentaire au sens orbifolde (ie : $f:\left(X_{1} \mid \Delta_{1}\right)^{d i v} \rightarrow(X \mid \Delta)^{d i v}$ est un morphisme orbifolde divisible, et $f_{*}\left(\Delta_{1}\right)=\Delta$ ). 
Dans ce cas, $f\left(\operatorname{Ratl}\left(X_{1} \mid \Delta_{1}\right)^{d i v}\right) \subset \operatorname{Ratl}(X \mid \Delta)^{d i v}$. Si donc $\left(X_{1} \mid \Delta_{1}\right)^{d i v}$ est $U R$ (resp. $R C$ ), il en est de même de $(X \mid \Delta)$.

L'inclusion $\left.f\left(\operatorname{Ratl}\left(X_{1} \mid \Delta_{1}\right)^{\operatorname{div}}\right) \subset \operatorname{Ratl}(X \mid \Delta)\right)^{\text {div }}$ étant stricte en général (voir 5.32 ci-dessous), les implications réciproques nécessitent un argument supplémentaire.

Lemme 5.31 Soit $X^{\prime} \in \mathcal{C}$ lisse et uniréglée (resp. rationnellement connexe), et $Z^{\prime} \subset X^{\prime}$ analytique fermé de codimension complexe au moins 2 . Pour tout $x^{\prime} \in X^{\prime}$ (resp. $\left.\left(x^{\prime}, y^{\prime}\right) \in X^{\prime} \times X^{\prime}\right)$, il existe une courbe rationnelle irréductible $R^{\prime}$ passant par $x^{\prime}$ (resp. contenant $x^{\prime}$ et $\left.y^{\prime}\right)$.

Démonstration : Supposons d'abord $X^{\prime}$ rationnellement connexe. Il existe alors, d'après [Ko-Mi-Mo 92], une courbe rationnelle irréductible $R^{\prime}$ passant par $x^{\prime}$ et $y^{\prime}$ et de fibré normal ample. Un argument simple de déformation montre alors que l'on peut déformer $R^{\prime}$ en une telle courbe évitant $Z^{\prime}$.

Lorsque $X^{\prime}$ est uniréglée (de dimension $n>0$ ), on considère son quotient rationnel $r^{\prime}: X^{\prime} \rightarrow Q^{\prime}$, qui est presque-holomorphe ([Ca 92], [Ko-Mi-Mo 92]) à fibres génériques lisses et $R C$ de dimension (strictement) positive. Soit $F^{\prime}$ une fibre générique : elle rencontre $Z^{\prime}$ en un ensemble de codimension au moins 2 dans $F$.

Le résultat précédent appliqué à $F^{\prime}$ permet donc de conclure

Montrons maintenant que si $(X \mid \Delta)^{d i v}$ est $U R$ (resp. $R C$ ), il en est de même de $\left(X_{1} \mid \Delta_{1}\right)^{d i v}$. (Remarquer que $\left(X_{1} \mid \Delta_{1}\right)$ n'est pas, en général, un quotient global).

Soit $Z \subset X$ le centre de la modification $g: X_{1} \rightarrow X$, et $Z^{\prime} \subset X^{\prime}$ son image réciproque dans $X^{\prime}$, qui y est de codimension au moins 2 puisque $f$ est finie. Puisque $(X \mid \Delta)^{\text {div }}$ est $U R$, il en est de même de $X^{\prime}$, et il existe donc une famille algébrique couvrante de courbes rationnelles de $X^{\prime}$ dont le membre générique évite $Z^{\prime}$. Leurs images par $f$ évitent donc $Z$, sont des courbes rationnelles de $(X \mid \Delta)^{d i v}$, et se relèvent donc par $g$ en une famille couvrante de courbes rationnelles de $\left(X_{1} \mid \Delta_{1}^{\text {div }}\right)$, qui est donc $U R$.

L'argument est identique pour la propriété $R C^{\text {div }}$

Exemple 5.32 Nous allons montrer que l'inclusion $f\left(\operatorname{Ratl}\left(X_{1} \mid \Delta_{1}\right)^{\text {div }}\right) \subset$ $\operatorname{Ratl}(X \mid \Delta)^{\text {div }}$ peut être stricte, même si $\left(X_{1} \mid \Delta_{1}\right)^{\text {div }}$ est minimum rendant $g:\left(X_{1} \mid \Delta_{1}\right)^{\text {div }} \rightarrow(X \mid \Delta)^{\text {div }}$ un morphisme orbifolde. 
Prenons $(X \mid \Delta):=\left(\mathbb{P}_{2} \mid \Delta\right)$, avec $\Delta:=(1-1 / 2) \cdot\left(D_{1}+D_{3}\right)+(1-1 / 3) \cdot D_{2}$, $D_{1}$ (resp. $\left.D_{2}\right)$ étant la droite d'équation affine $: x=0$ (resp. $\left.y=0\right)$, tandis que $D_{3}$ est une droite générique rencontrant la cubique rationnelle $R$ d'équation affine : $y^{2}=x^{3}$ en 3 points distincts a, $b, c$, dont le point à l'infini $a=(0,1,0)$ en coordonnées projectives. Alors $R$ est une courbe $\Delta^{\text {div }}$ rationnelle de $\mathbb{P}_{2}:=X$, puisqu'elle a un contact d'ordre 3 (resp. 2) avec $D_{2}$ (resp. $D_{1}$ ), de sorte que le diviseur orbifolde minimum $\Delta_{R}$ sur $R$ rendant l'inclusion normalisée $\left(R \mid \Delta_{R}\right) \rightarrow\left(\mathbb{P}_{2} \mid \Delta\right)$ un morphisme orbifolde divisible est $\Delta_{R}:=(1-1 / 2) \cdot(\{a\}+\{b\}+\{c\})$.

Soit alors $g: X_{1} \rightarrow \mathbb{P}_{2}=X$ l'éclatement du point de coordonnées affines $(0,0)$, et $E$ le diviseur exceptionnel de cet éclatement. Soit $R_{1}$ la transformée stricte de $R$ dans $X_{1}$. Elle a donc un ordre de contact avec $E$ (resp. la transformée stricte $D_{1}^{\prime}$ de $D_{1}$; resp. $D_{2}^{\prime}$ de $D_{2}$ ) égal à 2 (resp. 0 (sur E); resp. 1 ). La structure orbifolde minimum $\Delta_{1}^{\text {div }}$ sur $X_{1}$ faisant de $g:\left(X_{1} \mid \Delta_{1}\right) \rightarrow(X \mid \Delta)$ un morphisme orbifolde divisible est :

$\Delta_{1}^{\text {div }}:=(1-1 / 6) \cdot E+(1-1 / 2) \cdot\left(D_{1}^{\prime}+D_{3}^{\prime}\right)+(1-1 / 3) \cdot D_{2}^{\prime}$.

Tandis que la structure orbifolde minimum $\Delta_{R_{1}}^{\text {div }}$ sur $R_{1}$ faisant de l'inclusion $\left(R_{1} \mid \Delta_{R_{1}}\right) \rightarrow\left(X_{1} \mid \Delta_{1}\right)$ un morphisme orbifolde divisible est :

$\Delta_{R_{1}}^{\text {div }}:=(1-1 / 2) \cdot(\{d\}+\{a\}+\{b\}+\{c\})$, en identifiant $a, b, c$ avec leurs images réciproques dans $X_{1}$, tandis que $d:=\left(E \cap D_{2}^{\prime}\right)$. Il en résulte que $R_{1}$ n'est pas une $\Delta_{1}^{d i v}$-courbe rationnelle (mais elle reste cependant "elliptique").

On peut d'ailleurs vérifier que $R_{1}$ n'est pas non plus une courbe $\left(X_{1} \mid \Delta^{\prime}\right)^{Q_{-}}$ rationnelle si $g:\left(X_{1} \mid \Delta^{\prime}\right)^{Q} \rightarrow(X \mid \Delta)^{Q}$ est un morphisme orbifolde.

\subsection{Sections orbifoldes.}

Cette section tente de formuler le théorème de [G-H-S 03] dans le cadre des orbifoldes géométriques. Rappelons-le :

Théorème 5.33 ([G-H-S 03]) Soit $g^{\prime}: X^{\prime} \rightarrow B^{\prime}$ une fibration, avec $X^{\prime}, B^{\prime}$ projectives, lisses et connexes, et $B^{\prime}$ une courbe. Si la fibre générique de $g^{\prime}$ est $R C$, alors $g^{\prime}$ admet une section.

Dans le cas des quotients globaux, la situation se réduit encore au cas précédent :

Proposition 5.34 Soit $f: X^{\prime} \rightarrow(X \mid \Delta)$ un quotient global au sens de 5.15. On suppose qu'il existe un diagramme commutatif : 


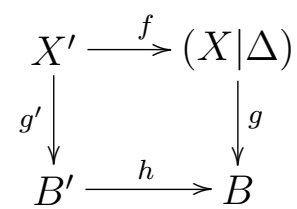

dans lequel les flèches verticales sont des fibrations, et où $B^{\prime}, B$ sont des lisses et connexes. Alors:

1. $K_{B^{\prime}} \leq h^{*}\left(K_{B}+\Delta(g, \Delta)^{d i v}\right)$.

2. Si g' a une section $\sigma^{\prime}$, alors : $K_{B^{\prime}}=h^{*}\left(K_{B}+\Delta(g, \Delta)^{\text {div }}\right)$, et $\Delta(g, \Delta)^{\text {div }}=\Delta(g, \Delta)^{Z}$. De plus :

3. Si $g^{\prime}$ a une section méromorphe $\sigma^{\prime}$, et si $B_{1}:=$ est la normalisée de $f \circ \sigma^{\prime}\left(B^{\prime}\right)$, munie de la plus petite structure orbifolde $\Delta_{B_{1}}^{\text {div }}$ faisant de l'inclusion normalisée $i:\left(B_{1} \mid \Delta_{B_{1}}\right)^{\text {div }} \rightarrow(X \mid \Delta)^{\text {div }}$ un morphisme, alors $g:\left(B_{1} \mid \Delta_{B_{1}}\right)^{d i v} \rightarrow\left(B \mid \Delta_{B}\right)^{\text {div }}$ est étale.

\section{Démonstration :}

1. Soit $b^{\prime} \in B^{\prime}$, et $e\left(b^{\prime}\right):=e$ l'indice de ramification de $h$ en $b^{\prime}$. Soit $b:=h\left(b^{\prime}\right) \in B$. Soit $x^{\prime} \in X^{\prime}$ tel que $g^{\prime}\left(b^{\prime}\right)=b^{\prime}$, et $x:=f\left(x^{\prime}\right)$. Soit $D$ une composante irréductible de $g^{-1}(b)$ contenant $x$, et $D^{\prime}$ une composante irréductible de $f^{-1}(D)$ contenant $x^{\prime}$.

Alors près de $x^{\prime}$ et de $x$, on a :

$t_{D} \cdot m_{\Delta}(D) \cdot D^{\prime}+\ldots=f^{*}\left(g^{*}(b)\right)=(g \circ f)^{*}(b)=\left(h \circ g^{\prime}\right)^{*}(b)=g^{\prime *}\left(e \cdot b^{\prime}\right)=e \cdot d \cdot D^{\prime}+\ldots$,

en désignant par $t_{D}$ (resp. $d$ ) la multiplicité de $D$ (resp. $D^{\prime}$ ) dans la fibre de $g$ au-dessus de $b$ (resp. de $g^{\prime}$ au-dessus de $b^{\prime}$ ).

Puisque, par définition, $m_{\Delta_{B}^{\text {div }}}(b)=\operatorname{pgcd}_{\{D\}}\left\{t_{D} \cdot m_{\Delta}(D)\right\}$, on voit que $e(b)$ divise $m_{\Delta_{B}^{\text {div }}}(b)$, ce qui est l'assertion 1 .

2. Si $g^{\prime}$ admet une section $\sigma^{\prime}$ telle que $\sigma^{\prime}\left(b^{\prime}\right)=x^{\prime}$, on a $: d=1$. Donc : $e=t_{D} \cdot m_{\Delta}(D) \geq m_{\Delta_{B}^{Z}}(b)$ divise $m_{\Delta_{B}^{\text {div }}}(b)$. Puisque $m_{\Delta_{B}^{\text {div }}}(b)$ divise (toujours) $m_{\Delta_{B}^{Z}}(b)$, on a l'égalité $: e=t_{D} \cdot m_{\Delta}(D) \geq m_{\Delta_{B}^{Z}}(b)=m_{\Delta_{B}^{Z}}(b)$, pour tout $b \in B$, ce qui est l'assertion 2.

3. On sait (d'après 5.16 et la remarque qui suit), que $f \circ \sigma^{\prime}: B^{\prime} \rightarrow$ $\left(B_{1} \mid \Delta_{B_{1}}\right)^{\text {div }}$ est étale au sens de 5.18.

Puisque la composée $h=\left(g \circ f \circ \sigma^{\prime}\right): B^{\prime} \rightarrow\left(B \mid \Delta_{B}\right)^{d i v}$ est étale (par 2. ci-dessus), on en déduit que $g:\left(B_{1} \mid \Delta_{B_{1}}\right)^{\text {div }} \rightarrow\left(B \mid \Delta_{B}\right)^{\text {div }}$ est aussi étale

Corollaire 5.35 Dans la situation du diagramme de 5.34, si les fibres de $g^{\prime}$ sont $R C$, alors $g^{\prime}$ a une section $\sigma^{\prime}$ (au sens de 5.37), et donc (d'après 5.34): 

5.18).

1. $K_{B^{\prime}}=h^{*}\left(K_{B}+\Delta(g, \Delta)^{d i v}\right)$ (autrement dit : $h$ est étale au sens de

2. $\Delta(g, \Delta)^{\text {div }}=\Delta(g, \Delta)^{Z}$.

3. $g:\left(B_{1} \mid \Delta_{B_{1}}\right)^{\text {div }} \rightarrow\left(B \mid \Delta_{B}\right)^{\text {div }}$ est étale au sens de 5.18 .

4. En particulier, si $\left(B \mid \Delta_{B}\right)^{\text {div }}$ est rationnelle, $(X \mid \Delta)$ div et $X^{\prime}$ sont $R C$.

Remarque 5.36 Il est clair qu'une fibration $f:(X \mid \Delta) \rightarrow B$ n'a en général pas de (multi)section au sens orbifolde (dans le sens suivant) si l'on munit sa base de la structure orbifolde au sens divisible (ie : de la plus grande structure orbifolde $\left(B \mid \Delta_{B}\right)^{\text {div }}$ telle que $f:(X \mid \Delta)^{\text {div }} \rightarrow\left(B \mid \Delta_{B}\right)^{\text {div }}$ soit un morphisme (orbifolde divisible)), et si l'on exige que la (multi)section $B^{\prime} \subset X$, une fois munie de la plus petite structure orbifolde $\Delta_{B^{\prime}}$ telle que l'inclusion normalisée $i:\left(B^{\prime} \mid \Delta_{B^{\prime}}\right) \rightarrow(X \mid \Delta)$ soit un morphisme orbifolde (même dans Georb $\left.^{Q}\right)$, induise une composée $f \circ i:\left(B^{\prime} \mid \Delta_{B^{\prime}}\right) \rightarrow\left(B \mid \Delta_{B}\right)$ qui soit étale.

Considérons par exemple la seconde projection $f_{0}: X_{0}:=\mathbb{P}_{1} \times \mathbb{P}_{1} \rightarrow$ $\mathbb{P}_{1}:=B$. On éclate d'abord $X_{0}$ aux trois points $(0,0),(0,1)$ et $(0, \infty)$. On obtient ainsi $X_{1}$ et sur $X_{1}$, six $(-1)$-courbes $F_{0}, F_{1}, F_{\infty}$ et $E_{0}, E_{1}, E_{\infty}$, où les $F_{i}$ sont les transformés stricts des fibres $f^{-1}(i)$, et $E_{i}$ le diviseur exceptionnel correspondant, image réciproque de $(0, i)$ pour $i=0,1, \infty$.

On éclate ensuite $X_{1}$ aux trois points $a_{i}:=\left(E_{i} \cap F_{i}\right)$. On obtient ainsi $X_{2}:=X$, et trois $(-2)$-courbes exceptionnelles sur $X$, notées $G_{i}$. On note encore $F_{i}, E_{i}$ les transformés stricts dans $X$, et $f: X \rightarrow \mathbb{P}_{1}:=B$ la composée de $f_{0}$ avec les deux éclatements précédents. On munit $X$ du diviseur orbifolde $\Delta:=(1-1 / 3)\left(F_{1}+F_{2}+F_{3}+E_{1}+E_{2}+E_{3}\right)+(1-1 / 2) \cdot\left(G_{1}+G_{2}+G_{3}\right)$.

Alors la base orbifolde divisible $\left(\Delta_{B}\right)^{\text {div }}$ de $f$ est nulle (puisque $\operatorname{pgcd}(2,3)=1)$. Donc toute multisection étale de $g$ doit être (par simpleconnexité orbifolde) une section. Puisque toute section $s: B \rightarrow X$ ne rencontre aucune des $G_{i}$ (qui ont multiplicité 2 comme fibres de $f$ ), la structure orbifolde $\left(\Delta_{B^{\prime}}\right)^{\text {div }}$ minimum d'une telle section est $\left(\Delta_{B^{\prime}}\right)^{\text {div }}:=$ $(1-1 / 3) \cdot(\{0\}+\{1\}+\{\infty\})$, qui n'est pas isomorphe à $(B \mid 0)$, et $\left(B^{\prime} \mid \Delta_{B^{\prime}}\right)$ div n'est pas même une courbe rationnelle orbifolde.

Remarquons que la base orbifolde $\left(\Delta_{B}\right)^{Q}$ de $f$ est $\left(\Delta_{B}\right)^{Q}:=(1-$ $1 / 2) \cdot(\{0\}+\{1\}+\{\infty\})$ (qui est rationnelle orbifolde, mais a pour groupe fondamental $\mathbb{Z}_{2} \times \mathbb{Z}_{2}$ ), et que $f$ n'a donc pas non plus de section orbfifolde dans la catégorie $G_{e o r b}{ }^{Q}$. Elle a, par contre, une 4-section orbifolde étale.

Les "sections" orbifoldes seront donc (sous réserve d'existence) des multisections. 
Definition 5.37 Soit $f:(X \mid \Delta) \rightarrow B$ une fibration sur une courbe lisse projective connexe $B$, avec $X$ une variété complexe compacte et connexe, et $(X \mid \Delta)$ lisse, entière. Soit $\left(B \mid \Delta_{B}\right)^{Z}$ la base orbifolde de $(f, \Delta)$ dans la catégorie Georb ${ }^{Z}$. C'est aussi la plus grande structure orbifolde sur $B$ pour laquelle $f$ est un morphisme orbifolde (non-classique).

Une section de $(f, \Delta)$ est alors une courbe irréductible normalisée $i$ : $B^{\prime} \rightarrow X$ (birationnelle sur son image) telle que $f \circ i: B^{\prime} \rightarrow B$ soit finie (ie : surjective), et telle que si $\Delta_{B^{\prime}}$ est le plus petit diviseur orbifolde sur $B^{\prime}$ tel que $i:\left(B^{\prime} \mid \Delta_{B^{\prime}}\right)^{Z} \rightarrow(X \mid \Delta)^{Z}$ soit un morphisme orbifolde, alors la composée $f \circ i:\left(B^{\prime} \mid \Delta_{B^{\prime}}\right) \rightarrow\left(B \mid \Delta_{B}\right)$ est un morphisme orbifolde étale (au sens de 5.18).

Nous allons déduire l'existence de sections pour les fibrations à fibres orbifoldes rationnellement connexe de la généralisation 5.39 de la conjecture suivante, due à Hassett-Tschinkel :

Conjecture 5.38 ([H-T 04]) Soit $g: X \rightarrow B$ une fibration, avec $X, B$ projectives, lisses et connexes, et $B$ une courbe. Si la fibre générique de $g$ est $R C$, et si $E \subset X$ est un sous-ensemble fini en chaque point duquel la fibre de $g$ est lisse et réduite, alors $g$ admet une section dont l'image contient $E$.

Conjecture 5.39 Soit $g:(X \mid \Delta) \rightarrow B$ une fibration, avec $X, B$ projectives, lisses et connexes, et $B$ une courbe. On suppose que la fibre orbifolde générique $\left(X_{b} \mid \Delta_{b}\right)$ de $g$ est lisse et $R C$, et que le support de $\Delta$ n'a pas de composante irréductible g-verticale (ie : contenue dans une fibre de g). Soit $E \subset X$ est un sous-ensemble fini en chaque point duquel la fibre de $f$ est lisse et réduite.

Alors $f$ admet une section $\sigma$ dont l'image contient $E$, et dont l'image $B_{1}$ est telle que l'inclusion : $i:\left(B_{1} \mid \Delta_{B_{1}}:=0\right)^{\text {div }} \rightarrow(X \mid \Delta)^{\text {div }}$ est un morphisme orbifolde. (De manière équivalente, pour toute composante irréductible $D_{j} d u$ support de $\Delta$ de multiplicité $m_{j}$ dans $\Delta$, et tout b de $B$, si $\sigma(b) \in D_{j}$, l'ordre de contact de $D_{j}$ avec $B_{1}$ en $\sigma(b)$ est divisible par $m_{j}$ ).

Remarque 5.40 Il est clair que la conjecture 5.39 fournit une section orbifolde de $g$ (au sens de 5.37) dans la situation particulière où $\Delta$ n'a pas de composante $g$-verticale.

Proposition 5.41 Soit $g:(X \mid \Delta) \rightarrow B$ une fibration, avec $X, B$ projectives, lisses et connexes, et $B$ une courbe. On suppose que la fibre orbifolde générique $\left(X_{b} \mid \Delta_{b}\right)$ de $g$ est $R C$. 
Admettons la conjecture 5.39.

1. Alors $g$ a une (multi)section orbifolde au sens de 5.37.

2. En particulier, si $(X \mid \Delta)$ est lisse et si $\left(B \mid \Delta_{g, \Delta}\right)^{Z}$ est rationnelle, alors $(X \mid \Delta)$ est $R C C$.

\section{Démonstration :}

Soit $\Delta_{B}:=\Delta_{g, \Delta}^{Z}=\sum_{b \in B}(1-1 / m(b)) .\{b\}=\sum_{k}\left(1-1 / m_{k}\right) .\left\{b_{k}\right\}$, avec : $m_{k}:=m\left(b_{k}\right):=\inf _{j}\left\{t_{j} \cdot m_{\Delta}\left(F_{j}\right)\right\}$, si $g^{*}\left(b_{k}\right)=\sum_{j} t_{j} . F_{j}$. Pour chacun des $b_{k}$, soit $j_{k}$ un indice tel que $t_{j_{k}} \cdot m_{\Delta}\left(F_{j_{k}}\right)=m_{k}$. Posons : $m_{k}(g):=t_{j_{k}}$, et $\Delta_{g}:=\sum_{k}\left(1-1 / m_{k}(g)\right) \cdot\left\{b_{k}\right\}$.

Il existe alors un revêtement orbifolde étale $: u: B^{\prime} \rightarrow\left(B \mid \Delta_{g}\right)^{23}$. On considère $g^{\prime}: X^{\prime}:=\widehat{X \times_{B} B_{1}} \rightarrow B_{1}$, déduit de $g$ par changement de base et normalisation. Alors l'image réciproque $F_{j_{k}}^{\prime}$ de chacun des $F_{j_{k}}$ est une composante réduite de la fibre de $g^{\prime}$ au-dessus de $b_{k}^{\prime}:=u^{-1}\left(b_{k}\right)$. Notons $f: X^{\prime} \rightarrow X$ la projection naturelle. Choisissant pour chaque $k$ un point lisse $E_{k}$ de $F_{j_{k}}^{\prime}$, et notant $E$ la réunion des $E_{k}$, on peut appliquer la conjecture 5.39 à $\left(X^{\prime} \mid f^{*}\left(\Delta^{h o r}\right)\right)$, où $\Delta^{\text {hor }}$ est la partie $g$-horizontale de $\Delta$. On obtient ainsi une section $\sigma^{\prime}$ de $g$ contenant $E$, et ayant les ordres de tangence requis le long de $f^{*}(\Delta)^{\text {hor }}$ ).

On vérifie immédiatemment que $B_{1}:=f\left(\sigma^{\prime}\left(B^{\prime}\right)\right)$ est une (multi)section orbifolde de $g:(X \mid \Delta) \rightarrow B$. La dernière assertion est alors immédiate

Remarque 5.42 On a vu (exemple 5.36) que si l'on considère, dans l'énoncé de 5.41 , non pas $\Delta_{B}^{Z}$, mais $\Delta_{B}^{\text {div }}$, la conclusion peut-être en défaut (i.e : il n'existe as néccessairement de section orbifolde de $g$ ). En fait, il peut alors même ne pas exister de $\Delta$-courbe rationnelle finie sur $B$, si $(B \mid \Delta)^{d i v}$ est rationnelle (voir l'exemple 5.43 ci-dessous).

Il est peut-être nécessaire dans cette situation, si l'on ne veut considérer que les bases orbifoldes divisibles, de borner les multiplicités pour que les fibres singulières (avec leurs multiplicités) soient (en un sens adéquat à définir) des spécialisations d'orbifoldes lisses et rationnellement connexes.

Exemple 5.43 Considérons par exemple la seconde projection $f_{0}: X_{0}:=$ $\mathbb{P}_{1} \times \mathbb{P}_{1} \rightarrow \mathbb{P}_{1}:=B$. On éclate d'abord $X_{0}$ aux trois points $(0,0),(0,1)$ et $(0, \infty)$. On obtient ainsi $u: X_{1} \rightarrow X_{0}$ et sur $X_{1}$, six $(-1)$-courbes $F_{0}, F_{1}, F_{\infty}$

\footnotetext{
${ }^{23}$ Sauf si $B=\mathbb{P}_{1}$, et si le support de $\Delta_{g}$ a 1 ou 2 éléments. Dans ce cas, on choisit simplement $B^{\prime}=B$. La courbe $B_{1}$ résultant de la construction qui suit ne sera pas une section orbifolde, mais sera une courbe $\Delta^{d i v}$-rationnelle, ce qui suffit pour nos applications.
} 
et $E_{0}, E_{1}, E_{\infty}$, où les $F_{i}$ sont les transformés stricts des fibres $f^{-1}(i)$, et $E_{i}$ le diviseur exceptionnel correspondant, image réciproque de $(0, i)$ pour $i=$ $0,1, \infty$. On note $g:=f_{0} \circ u: X_{1} \rightarrow \mathbb{P}_{1}$.

Soit $3<p<q$ deux entiers premiers entre eux. On munit $X_{1}$ de l'orbifolde $\Delta_{1}:=(1-1 / p) .\left(E_{0}+E_{1}+E_{\infty}\right)+(1-1 / q) \cdot\left(F_{0}+F_{1}+F_{\infty}\right) . I l$ est alors facile de voir que $\left(X_{1} \mid \Delta_{1}\right)^{Q}$ n'a pas de courbe rationnelle orbifolde $g$-horizontale $\left(B_{1} \mid \Delta_{B_{1}}\right)$. En effet $: \Delta_{g, \Delta_{1}}^{Q}=(1-1 / p) \cdot(\{0\}+\{1\}+\{\infty\})$, et donc : $\kappa\left(\left(\mathbb{P}_{1} \mid \Delta_{g, \Delta_{1}}\right)^{Q}\right)=1$. L'inclusion normalisée de $B_{1}$ dans $X_{1}$ composée avec $g$ fournirait alors un morphisme $g_{1}:\left(B_{1} \mid \Delta_{B_{1}}\right)^{Q} \rightarrow\left(\mathbb{P}_{1} \mid \Delta_{g, \Delta_{1}}\right)^{Q}$ qui induirait pour tout $N$ une inclusion $g_{1}^{*}: H^{0}\left(S^{N}\left(\Omega^{1}\left(\mathbb{P}_{1} \mid \Delta_{g, \Delta_{1}}\right)^{Q}\right) \rightarrow\right.$ $H^{0}\left(S^{N}\left(\Omega^{1}\left(B_{1} \mid \Delta_{B_{1}}\right)\right)\right.$. Ce qui implique que $\kappa\left(B_{1} \mid \Delta_{B_{1}}\right) \geq \kappa\left(\left(\mathbb{P}_{1} \mid \Delta_{g, \Delta_{1}}\right)^{Q}\right)=1$ et contredit la rationalité de $\left(B_{1} \mid \Delta_{B_{1}}\right)^{Q}$.

Remarquer que, par contre, $\Delta_{g, \Delta_{1}}^{d i v}=0$, puisque $\operatorname{pgcd}(p, q)=1$.

\subsection{Quotients globaux : l'implication $R E \Longrightarrow R C C$.}

Théorème 5.44 Soit $X^{\prime} \rightarrow(X \mid \Delta)$ un quotient global (voir 5.15). Si $(X \mid \Delta)$ est $R E_{\text {evit }}^{\text {div }}$, il est $\left(R C^{+}\right)^{d i v}$.

Démonstration : Il nous suffit de montrer que $X^{\prime}$ est $R C$, par 5.28. Nous pouvons supposer que le revêtement $f: X^{\prime} \rightarrow X$ est Galoisien de groupe $G$, fini (voir 5.15). Soit donc $g^{\prime}: X^{\prime} \rightarrow Y_{0}^{\prime}$ le quotient rationnel de $X^{\prime}$ ([Ca 92], [Ko-Mi-Mo 92]) : ses fibres sont $R C$ et sa base n'est pas uniréglée si ce n'est pas un point. Supposons donc $\operatorname{dim}\left(Y^{\prime}\right)>0$. Nous allons montrer que $Y^{\prime}$ est uniréglé, ce qui établira le résultat. La fibration $g^{\prime}$ est préservée par le groupe $G$. Soit $h_{0}: Y_{0}^{\prime} \rightarrow Y_{0}:=Y / G$ le quotient naturel. Il existe donc une application $g: X \rightarrow Y_{0}:=Y / G$ telle que $g \circ f=h \circ g^{\prime}$.

Il existe donc des modifications $u: X_{1} \rightarrow Y$ et $u^{\prime}: X_{1}^{\prime} \rightarrow X^{\prime}$, ainsi que des applications holomorphes : $f_{1}: X_{1}^{\prime} \rightarrow X_{1}$ et $h: Y^{\prime} \rightarrow Y$ telles que :

1. $h \circ g_{1}^{\prime}=g_{1} \circ f_{1}: X_{1}^{\prime} \rightarrow Y$, les applications $u^{\prime}$ et $f_{1}$ étant $G$-équivariantes.

2. Pour toute structure orbifolde $\Delta_{1}$ sur $X_{1}$ telle que $u_{*}\left(\Delta_{1}\right)=\Delta$ et telle que $u:\left(X_{1} \mid \Delta_{1}\right)^{d i v} \rightarrow(X \mid \Delta)^{d i v}$ soit un morphisme orbifolde, alors : (puisque $(X \mid \Delta)$ est $R E^{\text {div }}$ par hypothèse), la base orbifolde $\left(Y \mid \Delta_{Y}\right)$ est lisse et uniréglée, avec : $\Delta_{Y}:=\left(\Delta_{g_{1}, \Delta_{1}}\right)^{Z}$.

3. $h: Y^{\prime} \rightarrow Y$ est finie (mais $Y^{\prime}$ est seulement normal), et est un revêtement Galoisien de groupe $G$. 
Il existe donc sur $Y$ une unique structure orbifolde $\Delta_{Y}^{\prime}$ telle que $h: Y^{\prime} \rightarrow$ $Y$ ramifie exactement au-dessus de $\Delta_{Y}^{\prime}$. On peut, de plus, puisque $(X \mid \Delta)$ est, par hypothèse, $R E^{\text {div }}$, supposer que :

4. $\Delta_{Y}^{\prime}$ divise $\Delta_{Y}$ (i.e : $m_{\Delta_{Y}^{\prime}}(E)$ divise $m_{\Delta_{Y}}(E), \forall E \in W(Y)$ ).

En effet : soit $E \in W(Y)$ telle que $m^{\prime}(E)>m(E)$, notant $m^{\prime}(E)$ (resp. $\left.m^{\prime}(E)\right)$ la multiplicité de $E$ relative à $\Delta_{Y}^{\prime}$ (resp. $\left.\Delta_{Y}\right)$. Il existe donc $D \in$ $W\left(X_{1}\right)$ tel que $m^{\prime}(E)=t_{E}(D) \cdot m_{\Delta_{1}}(D)$, avec $g_{1}^{*}(E)=t_{E}(D) \cdot D+\ldots$, avec $t_{E}(D)>0$, i.e $: g_{1}(D)=E$.

On a alors 2 cas : ou bien $D$ est $u$-exceptionnel, ou bien $u(D)=D_{j} \in$ $W(X)$.

Dans le premier cas, on peut augmenter $m_{\Delta_{1}}(D)$ de telle sorte que $t_{E}(D) \cdot m_{\Delta_{1}}(D)$ soit un multiple de $m^{\prime}(E):\left(Y \mid \Delta_{Y}\right)$ reste uniréglée.

Dans le second cas, si $D^{\prime} \in W\left(X^{\prime}\right)$ et si $g_{1}^{\prime}\left(D^{\prime}\right)=E^{\prime}$, et $h\left(E^{\prime}\right)=E$, on a $:\left(h \circ g_{1}^{\prime}\right)^{*}(E)=g_{1}^{\prime *}\left(m^{\prime}(E) \cdot E^{\prime}\right)=\left(t_{E^{\prime}}^{\prime}\left(D^{\prime}\right) \cdot m^{\prime}(E)\right) \cdot D^{\prime}+\ldots=\left(g_{1} \circ f_{1}\right)^{*}(E)=$ $f_{1}{ }^{*}\left(t_{E}(D) \cdot D+\ldots\right)=\left(t_{E}(D) \cdot m_{\Delta}\left(D_{0}\right) \cdot D+\ldots\right.$, où $D_{0}:=u(D)$, puisque l'ordre de ramification de $f_{1}$ au-dessus de $D$ coincide avec celui de $f$ au-dessus de $D_{0}$.

On a donc (puisque $f_{1}$ et $h$ sont Galoisiens, et que seuls des diviseurs non $g_{1} \circ f_{1}$-exceptionnels interviennent) :

$t_{E^{\prime}}^{\prime}\left(D^{\prime}\right) \cdot m^{\prime}(E)=t_{E}(D) \cdot m_{\Delta}\left(D_{0}\right)=t_{E}(D) \cdot m_{\Delta_{1}}(D)=: m(E)$.

Donc : $m^{\prime}(E)$ divise $m(E)$.

En répétant un nombre fini de fois cette construction (au plus autant de fois que le nombre de diviseurs $D$ qui sont $u$-exceptionnels dans les images réciproques par $g_{1}$ des composantes du support de $\Delta_{1}$ ), on obtient la divisibilité annoncée.

Soit alors $B \subset Y$ une courbe $\Delta_{Y}$-rationnelle, membre générique d'une famille couvrante de telles courbes. C'est, a fortiori, une courbe $\Delta_{Y^{\prime}}^{\prime}$-rationnelle, et $\left(K_{Y}+\Delta_{Y}^{\prime}\right) \cdot B<0$. Soit $Z^{\prime} \subset Y^{\prime}$ le lieu singulier de $Y^{\prime}$, et $Z:=h\left(Z^{\prime}\right) \subset Y$ : $Z$ est de codimension au moins 2 dans $Y$. Puisque $(X \mid \Delta)$ satisfait la condition $R E_{\text {evit }}$, par hypothèse, nous pouvons supposer que $B$ évite $Z$. Soit $B^{\prime}$ une composante irréductible de $h^{-1}(B)$, membre d'une famille couvrante de $Y^{\prime}$, avec $h_{*}\left(B^{\prime}\right)=t . B, t>0$ : on a donc : $K_{Y^{\prime}} \cdot B^{\prime}=t$. $\left(K_{Y}+\Delta_{Y}^{\prime}\right) \cdot B<0$. Par [Mi-Mo 86], $Y^{\prime}$ est uniréglé, et le théorème démontré

Remarque 5.45 La démonstration montre que l'hypothèse que $(X \mid \Delta)$ est $R E_{\text {evit }}^{\text {div }}$ est très forte. Il serait intéressant de pouvoir l'affaiblir. 


\subsection{Uniréglage et Dimension Canonique}

Definition $5.46 S i(X \mid \Delta)$ est une orbifolde géométrique lisse, on pose : $\kappa_{+}(X \mid \Delta):=\max _{f}\{\kappa[Y \mid \Delta(f, \Delta)]\}, f:(X \mid \Delta) \rightarrow Y$ parcourant l'ensemble des applications méromorphes surjectives (ie : dominantes), avec $\operatorname{dim}(Y)>0$.

Donc $\kappa_{+}(X \mid \Delta)=-\infty$ signifie que :

$\kappa([Y \mid \Delta(f, \Delta)])=-\infty, \forall f:(X \mid \Delta) \rightarrow Y$.

La démonstration de l'énoncé suivant est facile :

Proposition 5.47 Soit $(X \mid \Delta)$ une orbifolde géométrique lisse, avec $X$ compacte et connexe.

1. Si $(X \mid \Delta)$ est uniréglée, alors $\kappa(X \mid \Delta)=-\infty$.

2. Si $(X \mid \Delta)$ est $R E$, alors $\kappa_{+}(X \mid \Delta)=-\infty$.

La conjecture suivante est la version orbifolde d'une conjecture standard (cas où $\Delta=0$ ) de la géométrie algébrique :

Conjecture 5.48 Soit $(X \mid \Delta)$ une orbifolde géométrique lisse, avec $X \in \mathcal{C}$ connexe. Si $\kappa(X \mid \Delta)=-\infty$, alors $(X \mid \Delta)$ est uniréglée.

Remarque 5.49 Le seul cas non-trivial avec $\Delta \neq 0$ dans lequel cette conjecture est connue est celui des surfaces projectives avec diviseur orbifolde logarithmique $(\Delta=S u p p(\Delta))$, par [K-M98]. Il s'agit donc dans ce cas de recouvrir $X$ par des courbes rationnelles $R$ rencontrant $\Delta$ en un seul point, en lequel $R$ est unibranche.

Théorème 5.50 Admettons la conjecture 5.48 précédente lorsque $\Delta=0$. Alors cette conjecture est encore valable pour tout quotient global $f: X^{\prime} \rightarrow$ $(X \mid \Delta)$.

Démonstration : En effet : $f^{*}\left(K_{X}+\Delta\right)=K_{X^{\prime}}$, de telle sorte que $\kappa(X \mid \Delta)=\kappa\left(X^{\prime}\right)=-\infty$. Donc $X^{\prime}$ est uniréglée (par 5.48), et donc aussi $(X \mid \Delta), \operatorname{par} 5.6$

Exemple 5.51 Soit à nouveau $(X \mid \Delta)$ l'exemple torique de 5.5.4. Il est rationnellement connexe (au sens orbifolde), et donc $\kappa_{+}(X \mid \Delta)=-\infty$. C'est un exemple dans lequel la conjecture 5.48 est vérifiée. 
Corollaire 5.52 Admettons la conjecture 5.48. Soit $(X \mid \Delta)$ une orbifolde géométrique lisse, avec $X \in \mathcal{C}$ connexe. Si $\kappa_{+}(X \mid \Delta)=-\infty$, alors $(X \mid \Delta)$ est RE.

Démonstration : Soit $R^{*}:=R_{(X \mid \Delta)}^{*}: X \rightarrow R^{*}(X \mid \Delta)$ la fibration construite en 5.26. Sa base est de dimension nulle, puisque $\kappa_{+}(X \mid \Delta)=-\infty$. Donc $(X \mid \Delta)$ est RE.

Remarque 5.53 Admettant une autre conjecture $\left(C_{n, m}^{\text {orb }}\right.$, voir $\left.\S 6.1\right)$, nous construirons en 6.14 une variante de la fibration $R^{*}$ : ses fibres orbifoldes générales ont $\kappa_{+}=-\infty$, et sa base orbifolde stable a $\kappa \geq 0$ (elle n'est donc pas uniréglée). Si 5.48 est vraie, ces deux fibrations coincident.

Exemple 5.54 Soit $X=\mathbb{P}^{2}$, et $\Delta=C$, avec $C$ une conique lisse. Les courbes rationnelles orbifoldes $R$ sont alors les courbes rationnelles (de degré $d)$, qui coupent $C$ en un unique point en lequel $R$ est unibranche.

Lorsque $d=1$, les tangentes à $C$ sont donc de tels exemples. Lorsque $d=2, R$ est une conique lisse osculatrice à $C$. On peut trouver de telles courbes $C$-rationnelles pour tous les degrés d. On devrait pouvoir en déduire que $\left(\mathbb{P}^{2} \mid C\right)$ est $R C$.

Lorsque les multiplicités sont finies, de nombreux nouveaux cas se présentent, même lorsque $n=2$, d'orbifoldes géométriques lisses $\left(\mathbb{P}^{2} \mid \Delta\right)$ qui sont Fano, et pour lesquelles la vérification de la conjecture 5.48 n'est pas immédiate.

Exemple 5.55 Soit $X=\mathbb{P}^{2}, \Delta=2 / 3\left(L_{3}+M_{3}\right)+4 / 5 L_{5}+6 / 7 . L_{7}$, où $L_{3}, M_{3}, L_{5}, L_{7}$ sont quatre droites en position générale. L'orbifolde géométrique lisse $\left(\mathbb{P}^{2} \mid \Delta\right)$ est Fano, puisque $2 / 3+1 / 5+1 / 7=1+1 / 105>1$.

Pour des petits degrés $N$, les $\Delta$-courbes rationnelles entières de $\mathbb{P}^{2}$ sont en nombre fini, et ne recouvrent pas $\mathbb{P}^{2}$. Il existe, par contre, des $\Delta$-coniques rationnelles (à multiplicités rationnelles) recouvrant génériquement $\mathbb{P}^{2}$ (voir l'exemple 5.5 .3 ci-dessus).

Pour des degrés $N$ multiples de 105, $N=k .105, k>0$, existe-t'il des $\Delta$-courbes rationnelles de degré $N$ dont tous les contacts avec $L_{d}, M_{d}$ (si $d=3$ ) sont d'ordre $d$, pour $d=3,5,7$ ? Un décompte de paramètres montre qu'il existe des familles de dimension $N(1 / 3+1 / 3+1 / 5+1 / 7-1)=k$ de telles courbes. Le problème est de démontrer que les polynômes de degré 
$N$ décrivant ces courbes sont bien premiers entre eux. Ce décompte de paramètres suggère d'ailleurs la connexité rationnelle par des familles de courbes rationnelles de degré suffisamment grand et divisible sans structure orbifolde pour toute orbifolde lisse, entière et finie $\left(\mathbb{P}_{n} \mid \Delta\right)$. Il serait intéressant d'avoir une approche plus conceptuelle (par déformation?) de ces problèmes.

\subsection{Appendice : Quotients méromorphes.}

Nous rappelons ici les résultats de [Ca04, appendice] (auquel nous renvoyons pour les démonstrations et plus de détails). Ils seront utilisés dans le présent texte dans les $§ 5.25$ et 11.5 .

Soit $X \in \mathcal{C}$, normal et connexe. On note $\mathcal{C}(X)$ ou $C h o w(X)$ la variété de Chow (ou espace des cycles) de $X$ construit dans [Ba75]. Pour $a \in \mathcal{C}(X)$, on note $Z_{a} \subset X$ le support du cycle paramétré par $a$.

On note $A \subset \mathcal{C}(X)$ un sous-ensemble (ensembliste). On supposera toujours que la famille $A$ est couvrante, c'est-à-dire que la réunion des $Z_{a}, a \in A$ est $X$. Soit $R_{A} \subset X \times X$ la relation d'équivalence pour laquelle deux points de $X$ sont équivalents si et seulement s'ils sont contenus dans $A$-chaine, ie : une réunion finie connexe de $Z_{a}, a \in A$.

Si $V \subset X$ est analytique fermé irréductible, on dira que $V \in A$ s'il existe $a \in A$ tel que $Z_{a}=V$.

Théorème 5.56 On suppose $A$ analytique fermé dans $\mathcal{C}(X)$. Il existe alors une unique fibration $q_{A}: X \rightarrow Q_{A}$, qui est presque-holomorphe (voir 7.16), et telle que pour $b \in Q_{A}$ général (générique si $A$ a un nombre fini de composantes), la fibre $X_{b}=q_{A}^{-1}(b)$ est la classe de $R_{A}$-équivalence de chacun de ses points. La fibration $q_{A}$ est appelée le A-quotient de $X$.

Definition 5.57 On dit que $A \subset \mathcal{C}(X)$ est Z-régulier si, pour tout $B \subset$ $\mathcal{C}(X)$, analytique fermé et irréductible, $A \cap B$ soit contient une intersection dénombrable d'ouverts de Zariski denses de $B$, soit est contenu dans une réunion dénombrable de sous-ensembles analytiques fermés stricts de $B$.

Si $A$ est Z-régulier, il existe une unique réunion finie ou dénombrable de sous-ensembles analytiques fermés irréductibles sans inclusions $B_{n}$ de $\mathcal{C}(X)$ (appelés les composantes de $A$ ), tels que, pour chaque $n, A \cap B_{n}$ contienne une intersection dénombrable d'ouverts de Zariski denses de $B_{n}$, et tels que $A$ soit contenu dans la réunion des $B_{n}$. On note $B$ la réunion des $B_{n}$. 
Théorème 5.58 Soit $A \subset \mathcal{C}(X), Z$-régulier et couvrant. Soit $q_{A}: X \rightarrow Q_{A}$ le $B$-quotient, $B$ étant la réunion des composantes de $A$ dans $\mathcal{C}(X)$. Si $b \in Q_{A}$ est général, et si $Z_{a}, a \in A$, rencontre $X_{b}$, alors $Z_{a} \subset X_{b}$.

Definition 5.59 Soit $A \subset \mathcal{C}(X), X \in \mathcal{C}$. On dit que $A$ est stable si, pour tout $V \subset X$ analytique fermé irréductible muni d'une fibration méromorphe dominante $g: V \rightarrow W$, alors $V \in A$ si :

1. Les fibres générales de $g$ sont dans $A$.

2. Il existe $Z \subset V, Z \in$ a tel que $g(Z)=W$.

Théorème 5.60 Soit $A \subset \mathcal{C}(X)$, Z-régulier et couvrant. Soit $q_{A}: X \rightarrow Q_{A}$ le $B$-quotient, $B$ étant la réunion des composantes de $A$ dans $\mathcal{C}(X)$. Si A est stable, et si $b \in Q_{A}$ est général, alors $X_{b} \in A$. 


\section{ADDITIVITÉ ORBIFOLDE}

On va rappeler ici certaines conjectures et résultats de $[\mathrm{Ca} 04, \S 4$, pp. 564574] auquel nous renvoyons pour les démonstrations et détails. Ces résultats sont techniquement essentiels pour établir les résultats principaux du présent texte (le "coeur" et sa décompostion).

\subsection{La conjecture $C_{n, m}^{o r b}$.}

Conjecture 6.1 (Conjecture $C_{n, m}^{\text {orb }}$ ) Soit $f:(Y \mid \Delta) \rightarrow S$ une fibration holomorphe, l'orbifolde géométrique $(Y \mid \Delta)$ étant lisse avec $Y$ compacte et connexe dans la classe $\mathcal{C}$.

Alors $\kappa(Y \mid \Delta) \geq \kappa\left(Y_{s} \mid \Delta_{s}\right)+\kappa(f \mid \Delta)$

On a noté $\left(Y_{s} \mid \Delta_{s}\right)$ la fibre orbifolde de $f$ au-dessus du point général $s \in$ $S$. (Remarquons que cette orbifolde géométrique est lisse, par le théorème de Sard, et $\kappa\left(Y_{s} \mid \Delta_{s}\right)$ est indépendant de $s \in S$, général, par le théorème de cohérence des images directes de Grauert, appliqué aux $f_{*}\left(m\left(K_{Y}+\Delta\right)\right)$, $m>0$ assez divisible).

Remarque 6.2 (1) Ici comme partout ailleurs ${ }^{24}$, les coefficients des composantes de $\Delta$ peuvent être supposés seulement rationnels dans [0,1], et pas nécessairement de la forme "standard" $(1-1 / m)$.

(2) Lorsque $f$ est nette (au sens de 3.8), on a aussi : $\kappa(f \mid \Delta)=$ $\kappa(S \mid \Delta(f, \Delta))$.

(3) Cette conjecture est évidemment la version orbifolde de la conjecture $C_{n, m}$ d'Iitaka, qui affirme que $\kappa(Y) \geq \kappa\left(Y_{s}\right)+\kappa(S)$ si $Y$ est projective.

\subsection{Le cas des fibrations de type général.}

Le résultat principal est ici le :

Théorème 6.3 Lorsque $f:(Y \mid \Delta) \rightarrow S$ est, de plus, une fibration de type général, la conjecture précédente $C_{n, m}^{o r b}$ est vraie, et on a alors :

$$
\kappa(Y \mid \Delta)=\kappa\left(Y_{s} \mid \Delta_{s}\right)+\operatorname{dim}(S) .
$$

\footnotetext{
${ }^{24}$ À l'exception des définitions du groupe fondamental, du revêtement universel, de la pseudométrique de Kobayashi, et des points entiers au sens des orbifoldes géométriques.
} 
Ce théorème est aisément déduit du suivant, adaptation au cadre orbifolde de résultats de E. Viehweg, initiés par T. Fujita et Y. Kawamata (Voir [Fuj78], [Kaw80], [Vie83]; un résultat similaire dans le cadre numérique est dû à Y. Kawamata [Kaw98]) :

Théorème 6.4 [Ca04, 4.13, p. 568] Soit $f: Y \rightarrow S$ une fibration, avec $Y, S$ lisses, $Y$ dans la classe $\mathcal{C}$, et $S$ projective. Soit $D=\sum m_{j} . D_{j}$ un diviseur entier et effectif sur $Y$ dont le support est à croisements normaux au-dessus du point générique de $S$. Soit $m>0$ un entier tel que $m \geq m_{j}$, pour tout $j$ tel que $D_{j}$ soit une composante $f$-horizontale de $D$ (ie : tel que $f\left(D_{j}\right)=S$ ). Alors : $f_{*}\left(m K_{Y / S}+D\right)$ est un faisceau cohérent faiblement positif ${ }^{25}$ sur $S$.

\section{Remarque 6.5}

1. Bien qu'obtenue par les mêmes méthodes que celles de [Vi 83], cette généralisation en étend considérablement le champ d'application.

2. Il résulte de 6.3 que si $f:(X \mid \Delta) \rightarrow Y$ est une fibration de type général (ie : la base orbifolde d'un modèle holomorphe net est de type général), alors les fibres génériques orbifoldes de deux modèles nets holomorphes de $f$ ont la même dimension canonique, égale à la différence entre $\kappa(X \mid \Delta)$ et $\operatorname{dim}(Y)$. Nous verrons en fait (en 7.17) un résultat plus précis : une telle fibration est presque-holomorphe.

3. Dans le Lemme 4.10, p.567 de [Ca04], l'hypothèse (par exemple) que $g_{*}(E)$ est localement libre a été omise, comme me l'a signalé O. Debarre. Cette hypothèse est difficilement vérifiable en pratique, mais on a la version suivante, qui couvre les applications présentes:

Proposition 6.6 Soit $f: Y \rightarrow S$ une fibration avec $Y$ compact, normal et connexe. Soit $A$ un $\mathbb{Q}$-diviseur ample sur $S$ et $D$ un fibré en droites sur $Y$ tel que $f_{*}(D)$ soit faiblement positif. Alors $\kappa\left(Y, D+f^{*}(A)+E\right)=$ $\kappa\left(Y_{s}, D_{s}\right)+\operatorname{dim}(S)$, pour $E$ un diviseur effectif $f$-exceptionnel adéquat sur $Y$. $S^{\prime}$ il existe un morphisme birationnel $v: Y \rightarrow Y^{\prime}$ contractant tous les diviseurs $f$-exceptionnels de $Y$, et un fibré en droites $D^{\prime}$ sur $Y^{\prime}$ tel que $D=v^{*}\left(D^{\prime}\right)$, on peut prendre $E=0$.

Lorsque $f$ est nette (c'est la situation présente, et aussi celle considérée dans [Ca04]), l'hypothèse de la seconde assertion de la proposition est satisfaite (et le lemme 4.10 peut donc bien être appliqué tel quel).

\footnotetext{
${ }^{25}$ Voir [Vi83] pour cette notion, due à E. Viehweg. Des rappels se trouvent aussi dans $[\mathrm{Ca} 04, \S 4]$
} 
Démonstration : (C'est, sous une forme simplifiée, celle de 4.6 et de 4.3 ci-dessus). Il suffit de montrer que $H^{0}\left(Y, m\left(L+E+f^{*}(A)\right) \neq 0\right.$ pour $E$ et $m>0$ adéquats. Par hypothèse, $H^{0}\left(S, S^{m}(F) \otimes m A\right) \neq 0$, si $S^{m}(F)$ est le bidual de $\operatorname{Sym}^{m}\left(f_{*}(D)\right)$. Une section non nulle de ce faisceau fournit donc une section de $m\left(D+f^{*}(A)\right)$ ayant des pôles sur un diviseur $f$-exceptionnel de $Y$ (on suppose $m A$ entier). D'où la première assertion. La seconde assertion résulte de ce que, sous l'hypothèse de contractibilité de l'énoncé, toute section de $m\left(D+f^{*}(A)\right)$ définie sur le complémentaire du lieu exceptionnel de $f$ se prolonge à $Y$ tout entier.

\subsection{Première application : $\kappa=0$.}

Notre première application du théorème 6.3 est l'exemple fondamental suivant d'orbifolde géométrique lisse "spéciale" (voir aussi définition 4.18 pour ce terme) :

Théorème 6.7 Soit $f:(Y \mid \Delta) \rightarrow S$ une fibration holomorphe, l'orbifolde géométrique $(Y \mid \Delta)$ étant lisse avec $Y$ compacte et connexe dans la classe $\mathcal{C}$. Si $\kappa(Y \mid \Delta)=0$, alors $(Y \mid \Delta)$ est "spéciale" (ie : il n'existe pas de fibration de type général $f:(Y \mid \Delta) \rightarrow X)$.

Démonstration : Soit $f:(Y \mid \Delta) \rightarrow X$ une telle fibration.

On a donc $\operatorname{dim}(S)>0$, et (c'est immédiat) $: \kappa\left(Y_{s} \mid \Delta_{s}\right) \geq 0$.

Donc : $0=\kappa(Y \mid \Delta)=\kappa\left(Y_{s} \mid \Delta_{s}\right)+\operatorname{dim}(S) \geq \operatorname{dim}(S)>0$, par 6.3. Contradiction•

Le même argument fournit un résultat un peu plus général :

Théorème 6.8 Soit $f:(Y \mid \Delta) \rightarrow S$ une fibration holomorphe de type général, l'orbifolde géométrique $(Y \mid \Delta)$ étant lisse avec $Y$ compacte et connexe dans la classe $\mathcal{C}$.

Si $\kappa(Y \mid \Delta) \geq 0$, alors $\operatorname{dim}(S) \leq \kappa(Y \mid \Delta)$, et on a égalité si et seulement si $\kappa\left(Y_{s} \mid \Delta_{s}\right)=0$, pour la fibre orbifolde générale.

\section{Remarque 6.9}

1. Lorsque $\kappa(Y \mid \Delta)=-\infty$, l'inégalité précédente tombe en défaut (déjà pour $\Delta=0$ : prendre $Y=\mathbb{P}^{r} \times X$, où $X$ est une variété de type général de dimension strictement positive arbitraire)

2. Rappelons que, dans les théorèmes 6.7 et 6.8 précédents, les multiplicités de l'orbifolde géométrique lisse $(X \mid \Delta)$ sont des rationnels $a_{j}, 0<a_{j} \leq$ $1, \forall j$. 
Corollaire 6.10 Soit $(X \mid \Delta)$ lisse et Fano (ie : $-\left(K_{X}+\Delta\right)$ est ample sur $X)$. Alors $(X \mid \Delta)$ est spéciale.

Démonstration : Soit $H$ une section lisse de $-m\left(K_{X}+\Delta\right)$ intersectant transversalement $\Delta$. Soit $\Delta^{\prime}:=\Delta+(1 / m)$. $H$. Alors : $\left(X \mid \Delta^{\prime}\right)$ est lisse, et $K_{X}+\Delta^{\prime}$ est de $\mathbb{Q}$-torsion, donc $\kappa\left(X \mid \Delta^{\prime}\right)=0$. Donc $\left(X \mid \Delta^{\prime}\right)$ est spéciale, par 6.7. Donc aussi $(X \mid \Delta)$, puisque $\Delta \leq \Delta^{\prime}$

Le même argument fournit l'exemple suivant (suggéré par une question de M. Mustaţa) :

Exemple 6.11 Soit $X$ une variété torique de fibré anticanonique $D$, et $\Delta \leq D$. Alors $(X \mid \Delta)$ est spéciale. En effet : le diviseur anticanonique, complémentaire de l'orbite ouverte, est à croisements normaux.

Un cas particulier utilisé dans la suite est le suivant :

Exemple 6.12 Soit $(Y \mid \Delta):=\left(\mathbb{P}^{r} / D_{r}\right)$ l'orbifolde géométrique (logarithmique) lisse de l'exemple 2.20, obtenue de $\mathbb{P}^{r}$ en munissant l'hyperplan à l'infini de la multiplicité $+\infty$. Cette orbifolde géométrique (torique) est donc spéciale.

\subsection{Le quotient $\kappa$-rationnel (conditionnel)}

Lemme 6.13 On suppose dans ce lemme que $C_{n, m}^{\text {orb }}$ est vraie. (Voir 6.1).

Soit $(X \mid \Delta)$ une orbifolde géométrique lisse, avec $X \in \mathcal{C}$.

Soit $f:(X \mid \Delta) \rightarrow Y$ et $g:(X \mid \Delta) \rightarrow Z$ deux fibrations avec $\operatorname{dim}(Y)>0$ et $\operatorname{dim}(Z)>0$, telles que $\kappa(f \mid \Delta) \geq 0$ et $\kappa(g \mid \Delta) \geq 0$.

Il existe alors une fibration $h:(X \mid \Delta) \rightarrow V$ telle que $\kappa(h \mid \Delta) \geq 0$ qui domine $f$ et $g$ (ie : il existe $u: V \rightarrow Y$ et $v: V \rightarrow Z$ telles que $v \circ h=g$ et $u \circ h=f)$.

Démonstration : On peut supposer $f$ et $g$ holomorphes, nettes, avec bases orbifoldes lisses. Soit $V \subset Y \times Z$ l'image du morphisme produit $h$ : $f \times g: X \rightarrow Y \times Z$ défini par $h(x)=(f(x), g(x))$. On note $u: V \rightarrow Y$ et $v: V \rightarrow Z$ les projections naturelles, telles que $u \circ h=f$, et $v \circ h=g$. Observons que $h$ est bien une fibration, puisque $V_{y}=g\left(X_{y}\right)$ est connexe, pour tout $y \in Y$. 
On peut encore, quitte à modifier encore $X, Y$ et $Z$, supposer la fibration $h:(X \mid \Delta) \rightarrow V$ nette et à base orbifolde lisse, et supposer aussi (par 3.6) que : $\Delta(f, \Delta)=\Delta(u, \Delta(h, \Delta))$.

La famille $g\left(X_{y}\right)=\left(V_{y}\right)_{y \in Y}$ forme une famille couvrante de sous-variétés de $Z$. Notons $\Delta_{Z}:=\Delta(g, \Delta)$. L'orbifolde géométrique stable $\left[\left(Z \mid \Delta_{Z}\right)_{V_{y}}\right]$ induite par restriction est donc bien définie, et est telle que $\kappa\left(\left[\left(Z \mid \Delta_{Z}\right)_{V_{y}}\right]\right) \geq 0$, puisque $\kappa(g \mid \Delta)=\kappa(Z \mid \Delta(g, \Delta))=\kappa\left(Z \mid \Delta_{Z}\right) \geq 0$, par hypothèse.

La fibration $v:(V \mid \Delta(h, \Delta)) \rightarrow Y$ a pour fibre orbifolde générique $(V \mid \Delta(h, \Delta))_{V_{y}} \cong\left[\left(Z /\left(\Delta_{Z}\right)_{V_{y}}\right]\right.$. On vient donc de voir que $\kappa\left((V \mid \Delta(h, \Delta))_{V_{y}} \geq\right.$ 0 . Par hypothèse $\kappa(Z \mid \Delta(v, \Delta(h, \Delta)))=\kappa(Z \mid \Delta(v \circ h, \Delta))=\kappa\left(Z \mid \Delta_{Z}\right) \geq 0$.

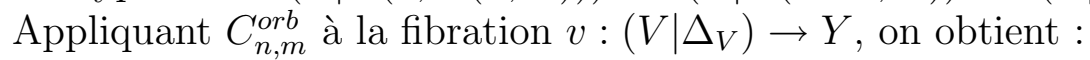

$\kappa\left(V \mid \Delta_{V}\right) \geq 0$

Corollaire 6.14 On suppose que $C_{n, m}^{\text {orb }}$ est vraie. (Voir 6.1).

Soit $\left(X \mid \Delta_{X}\right)$ une orbifolde géométrique lisse avec $X \in \mathcal{C}$, et $\operatorname{dim}(X)>0$.

Il existe une unique fibration $r_{X \mid \Delta}^{+}:(X \mid \Delta) \rightarrow R^{+}(X \mid \Delta)$ telle que :

1. $\kappa\left(\left[R^{+}(X \mid \Delta) \mid \Delta\left(r_{X \mid \Delta}^{+}, \Delta\right)\right]\right) \geq 0$.

2. $\kappa_{+}(X \mid \Delta)_{r}=-\infty$, si $\left[(X \mid \Delta)_{r}\right]$ est la fibre orbifolde stable générique de $r_{X \mid \Delta_{X}}^{+}$

Cette fibration, bien définie à équivalence biméromorphe près sur $(X \mid \Delta)$, est appelée le $\kappa$-quotient rationnel (conditionnel) de $(X \mid \Delta)$.

Démonstration : L'unicité est claire, puisque $\kappa(h \mid \Delta)=-\infty$, pour toute fibration $h:(X \mid \Delta) \rightarrow T$ telle que $\operatorname{dim}\left(h\left(X_{r}\right)\right)>0$ pour $r \in R^{+}(X \mid \Delta)$ générique.

Etablissons l'existence. Si $\kappa_{+}(X \mid \Delta)=-\infty$, on choisit pour $r_{X \mid \Delta}^{+}$l'application constante sur un point. Sinon, on choisit une fibration $f:(X \mid \Delta) \rightarrow Z$ avec $\operatorname{dim}(Z)>0$ maximum, telle que $\kappa(f \mid \Delta) \geq 0$. Le lemme précédent montre que $f$ domine toute fibration $g:(X \mid \Delta) \rightarrow T$ telle que $\kappa(g \mid \Delta) \geq 0$.

Il reste à montrer que $\kappa_{+}\left(\left[(X \mid \Delta)_{z}\right]\right)=-\infty$, pour $z \in Z$ générique.

Sinon, par récurrence sur $\operatorname{dim}(X)>0$, toute fibre générale $\left[(X \mid \Delta)_{z}\right]$ de $f$ admet un (unique) $\kappa$-quotient rationnel (conditionnel) $r_{z}^{+}:(X \mid \Delta)_{z} \rightarrow R_{z}^{+}$, holomorphe sur un modèle biméromorphe adéquat de $f$, et dont la famille des fibres forme donc une composante irréductible de $\mathcal{C} h o w\left(X_{z}\right)$. Il résulte alors (par des arguments exactement similaires) de la démonstration de 7.20 qu'il existe des fibrations $r^{+}:(X \mid \Delta) \rightarrow V$ et $s: V \rightarrow Z$ telles que :

1. $s \circ r=f:(X \mid \Delta) \rightarrow Z$.

2. $r_{\mid X_{z}}^{+}=r_{z}^{+}$, pour $z \in Z$ général. 
En particulier, à la fois la base orbifolde stable et la fibre orbifolde stable de $s:\left(V \mid \Delta\left(r^{+}, \Delta\right)\right) \rightarrow Z$ ont une dimension canonique positive ou nulle. Il résulte alors de $C_{n, m}^{\text {orb }}$ que $\kappa\left(V \mid \Delta\left(r^{+}, \Delta\right)\right) \geq 0$. Ce qui contredit la maximalité de $\operatorname{dim}(Z)$, puisque $\operatorname{dim}(V)>\operatorname{dim}(Z)$, par construction. Les fibres orbifoldes génériques de $f$ ont donc bien $\kappa_{+}=-\infty$.

\section{Remarque 6.15}

1. On a donc: $R^{+}(X \mid \Delta)=(X \mid \Delta)$ si et seulement si $\kappa(X \mid \Delta) \geq 0$, et dans ce cas, $M(X \mid \Delta)=(X \mid \Delta)$ si et seulement si $\kappa(X \mid \Delta)=\operatorname{dim}(X) \geq 0$.

2. Il peut se faire que $r_{\left(X \mid \Delta_{X}\right)}^{+}$ne soit pas presque-holomorphe lorsque $\Delta \neq 0$. Par exemple si $X=\mathbb{P}^{2}$, et si $\Delta$ est l'orbifolde géométrique logarithmique (multiplicités $+\infty$ ) dont le support consiste en deux droites (projectives, distinctes). Ceci est cependant peut-être particulier aux orbifoldes géométriques logarithmiques.

Ici encore, $r_{\left(X \mid \Delta_{X}\right)}^{+}$jouit de la propriété de fonctorialité suivante :

Lemme 6.16 Soit $f:\left(X \mid \Delta_{X}\right) \rightarrow\left(Y \mid \Delta_{Y}\right)$ est un morphisme dans la catégorie méromorphe des orbifoldes géométriques lisses. On suppose que $X \in \mathcal{C}$.

Notons (pour simplifier les notations) $\left[R_{X}^{+} \mid \Delta_{R_{X}^{+}}\right]$et $\left[R_{Y}^{+} \mid \Delta_{R_{Y}^{+}}\right]$les bases orbifoldes stables de $r_{\left(X \mid \Delta_{X}\right)}^{+}$et de $r_{\left(Y \mid \Delta_{Y}\right)}^{+}$respectivement.

Il existe alors un (unique) morphisme $r_{f}^{+}:\left[R_{X}^{+} \mid \Delta_{R_{X}^{+}}\right] \rightarrow\left[R_{Y}^{+} \mid \Delta_{R_{Y}^{+}}\right]$tel que : $r_{f}^{+} \circ r_{\left(X \mid \Delta_{X}\right)}^{+}=r_{\left(Y \mid \Delta_{Y}\right)}^{+} \circ f$.

Démonstration : Notons $F_{X}$ la fibre orbifolde générique de $r_{\left(X \mid \Delta_{X}\right)}^{+}, R_{Y}^{+}$ la base orbifolde stable de $r_{\left(Y \mid \Delta_{Y}\right)}^{+}$, et $r_{Y}^{+}:=r_{\left(Y \mid \Delta_{Y}\right)}^{+}$. Alors $r_{Y}^{+}: F_{X} \rightarrow R_{Y}^{+}$ définit une famille couvrante de $R_{Y}^{+}$. Il s'agit de montrer que $\operatorname{dim}\left(F_{X}\right)=0$. Supposons le contraire. L' orbifolde géométrique obtenue par restriction de $\left[\Delta\left(r_{Y}^{+}, \Delta_{X}\right)\right]$ à $r_{Y}^{+}\left(F_{X}\right)$ a donc $\kappa=-\infty$, puisque quotient de $F_{X}$ telle que $\kappa_{+}\left(F_{X}\right)=-\infty$. Puisque cette famille est couvrante, ceci contredit $\kappa\left(R_{Y}^{+} \mid \Delta\left(r_{Y}^{+}, \Delta_{Y}\right)\right) \geq 0$ 


\section{FIBRATIONS DE TYPE GÉNÉRAL.}

Notation 7.1 Désormais ${ }^{26},(Y \mid \Delta)$ désignera une orbifolde géométrique lisse avec $Y$ compacte et connexe.

On dira que $Y \in \mathcal{C}$ si $Y$, compact, normal et connexe, est biméromorphe à une variété Kählérienne compacte.

On notera $f:(Y \mid \Delta) \rightarrow X$ une application méromorphe surjective.

\subsection{Fibrations de type général : définition, stabilité par composition}

Definition 7.2 Soit $f:(Y \mid \Delta) \rightarrow X$ une fibration méromorphe avec $X, Y$ compacts et iréductibles, et $(Y \mid \Delta)$ une orbifolde géométrique lisse. On dit que $(f \mid \Delta)$ (ou $f$ s'il n' y a pas d'ambiguité sur $\Delta$ ) est une fibration de type général si $\kappa(f \mid \Delta)=\operatorname{dim}(Y)>0$.

Si $(Y \mid \Delta)$ est une orbifolde géométrique lisse, avec $Y$ compacte et connexe. On note $F T G(Y \mid \Delta)$ l'ensemble des classes d'équivalence biméromorphe de fibrations de type général sur $(Y \mid \Delta)$. Si $f$ est une telle fibration on notera $[f]$ sa classe d'équivalence dans $F T G(Y \mid \Delta)$.

Cet ensemble ne dépend donc que de la classe d'équivalence biméromorphe de $(Y \mid \Delta)$.

Proposition 7.3 Soit $g:\left(Z \mid \Delta_{Z}\right) \rightarrow\left(Y \mid \Delta_{Y}\right)$ un morphisme surjectif d'orbifoldes géométriques lisses ( $Y$ et $Z$ étant compactes et connexes), et $f:(Y \mid \Delta) \rightarrow X$ holomorphe surjective. Si $f$ est de type général, la composée $f \circ g$ l'est aussi. La propriété subsiste lorsque $f$ et $g$ sont méromorphes.

Démonstration : Si $f$ est de type général, $\kappa\left(Y, L_{f}\right)=p=\operatorname{dim}(X)>$ 0 . Or $L_{f \circ g}=g^{*}\left(L_{f}\right)$. Donc $\kappa\left(Z, L_{f \circ g}\right)=\kappa\left(Z, g^{*}\left(L_{f}\right)\right)=\kappa\left(Y, L_{f}\right)$. D'où la première assertion. La seconde assertion se démontre de la même manière, puisque la dimension canonique des faisceaux $L_{f}$ est un invariant biméromorphe des orbifoldes géométriques lisses.

\footnotetext{
${ }^{26}$ C'est-à-dire : dans la présente $\S 7$.
} 


\subsection{Faisceaux de Bogomolov et fibrations de type général.}

Definition 7.4 Soit $(Y \mid \Delta)$ une orbifolde géométrique lisse, et $L \subset \Omega_{X}^{p}$, avec $p>0$, un sous-faiscau cohérent de rang 1. Rappelons que, d'après 2.24, $\kappa(Y \mid \Delta, L) \leq p$.

On dit que $L$ est un Faisceau de Bogomolov si $\kappa(Y \mid \Delta, L)=p$.

Deux tels faisceaux sont équivalents s'ils coincident sur un ouvert non vide de $X$. On note $\operatorname{Bog}(Y \mid \Delta)$ l'ensemble (éventuellement vide) des classes d'équivalence de tels faisceaux. On note $[L]$ la classe d'équivalence de $L$.

Si $f:(Y \mid \Delta) \rightarrow X$ est une fibration méromorphe, de faisceau $L_{f}:=$ $f^{*}\left(K_{X}\right)$ au point générique de $Y$, alors le corollaire 4.3 montre que $L_{f}$ est un faisceau de Bogomolov si et seulement si $f$ est de type général. D'où une application naturelle (clairement injective) $: F:=F_{(Y \mid \Delta)}: F G T(Y \mid \Delta) \rightarrow$ $\operatorname{Bog}(Y \mid \Delta)$.

Il résulte du corollaire 2.24 que cette application est surjective lorsque $Y$ est biméromorphe à une variété Kählérienne compacte (ie : dans a classe $\mathcal{C}$ de Fujiki). D'où le :

Théorème 7.5 Si $(Y \mid \Delta)$ est une orbifolde géométrique lisse, avec $Y$ dans la classe $\mathcal{C}$, l'application $F_{(Y \mid \Delta)}: F G T(Y \mid \Delta) \rightarrow B o g(Y \mid \Delta)$ précédente est bijective.

\subsection{Restriction à une sous-variété générique.}

On étend au cas des orbifoldes géométriques géométriques certains des résultats de [Ca 04].

Pour les notions de restriction au sens orbifolde, voir $§ 2.13$.

Proposition 7.6 Soit $f:(Y \mid \Delta) \rightarrow X$ une application méromorphe de type général, avec $(Y \mid \Delta)$ lisse, $Y$ compacte et connexe. Soit $V \subset Y$ une sous-variété non contenue dans le support de $\Delta$, et non contenue dans le lieu d'indétermination de $f$. Si $f(V)=X$, alors $f_{V}:\left(V \mid \Delta_{V}\right) \rightarrow X$ est aussi de type général, pour tout choix de la restriction $f_{V}$.

Démonstration : Soit $p:=\operatorname{dim}(X)>0$. Soit $\left(V^{\prime} \mid \Delta_{V^{\prime}}\right)$ une restriction arbitraire de $\Delta$ à $V$. Voir $\S 2.13$ pour cette notion. On obtient $L_{f_{V}} \subset \Omega_{V^{\prime}}^{p}$ en composant l'inclusion $L_{f}:=f^{*}\left(K_{X}\right) \subset \Omega_{Y}^{p}$ avec la restriction naturelle $\Omega_{Y}^{p} \rightarrow \Omega_{V^{\prime}}^{p}$. On en déduit l'inégalité $: p:=\kappa(f \mid \Delta) \leq \kappa\left(f_{V} \mid \Delta_{V}\right)$, et l'assertion. 
Definition 7.7 Soit $Y$ une variété complexe connexe. Une famille couvrante $\left(Z_{t}\right)_{t \in T}$ de sous-variétés de $Y$ est un quadruplet $(Z, T, g, h)$ constitué d'une variété complexe connexe $Z$, et de deux applications holomorphes propres et surjectives $h: Z \rightarrow T$, et $g: Z \rightarrow Y, h$ ayant des fibres connexes.

On note alors, pour $t \in T, Z_{t}:=\left(h^{-1}(t)\right) \subset Z$, et $Y_{t}:=g\left(Z_{t}\right):$ c'est un sous-ensemble analytique compact, irréductible si $t \in T$ est générique. On suppose aussi que la restriction $g_{t}: Z_{t} \rightarrow Y_{t}$ de $g$ à $Z_{t}$ est biméromorphe, pour $t \in T$ générique.

On note enfin $: X_{t}:=f\left(Y_{t}\right) \subset X$, et $f_{t}: Y_{t} \rightarrow X_{t}$ la restriction de $f$, si $f: Y \rightarrow X$ est holomorphe.

On dit que la famille $Z_{t}$ se factorise par la fibration $f: Y \rightarrow X$ s'il existe une application méromorphe $m: T \rightarrow X$ telle que $m \circ h=f \circ g$.

Théorème 7.8 Si $(Y \mid \Delta)$ est une orbifolde géométrique lisse, et si $f$ : $(Y \mid \Delta) \rightarrow X$ est une fibration méromorphe, la restriction de $f$ au membre générique d'une famille couvrante de sous-variétés de $Y$ est de type général si cette famille ne se factorise pas par $f$.

Démonstration : On peut remplacer $(Y \mid \Delta)$ par $\left(Y^{\prime} \mid \Delta^{\prime}\right)$ ci-dessus, et supposer de plus que $f$ est holomorphe. Soit alors $t \in T$ générique, et $d_{t}$ : $X_{t}^{\prime} \rightarrow X_{t}$ une désingularisation de $X_{t}$.

Alors on a une injection naturelle de faisceaux sur $X_{t}^{\prime}: d_{t}^{*}\left(K_{X}\right) \subset K_{X_{t}^{\prime}}$ qui fournit une injection de faisceaux sur $Y_{t}:\left(f_{t}^{\prime}\right)^{*}\left(K_{X}\right)=L_{f \mid Y_{t}} \subset L_{f_{t}}$, notant $f_{t}^{\prime}:=\left(d_{t}\right)^{-1} \circ f_{t}: Y_{t} \rightarrow X_{t}^{\prime}$.

On en déduit que $\kappa\left(f_{t} \mid \Delta_{Y_{t}}\right)=\kappa\left(Y_{t}, L_{f_{t}}\right) \geq \kappa\left(Y_{t},{\overline{L_{f, m}}}_{Y_{t}}\right)$, ceci pour tout $m>0$.

Puisque $t \in T$ est générique, puisque le système linéaire $\left|L_{f, m}\right|$ définit $f: Y \rightarrow X$ pour $m$ assez grand, et puisque $f$ est de type général, le système linéaire $\left|L_{f, m \mid Y_{t}}\right|$ définit $f_{t}: Y_{t} \rightarrow X_{t}$, qui est donc bien de type général sur $\left(Y_{t} \mid \Delta_{Y_{t}}\right)$.

Remarque 7.9 L'argument précédent montre, plus précisément, que $f_{V}$ : $\left(V \mid \Delta_{V}\right) \rightarrow W:=f(V)$ est de type général si $V$ rencontre la fibre générique de $f$ (et n'est pas contenue dans le support de $\Delta$ ).

Rappelons la :

Definition 7.10 Un point $t \in T$ est dit général s'il appartient à un sousensemble de $T$ contenant une intersection dénombrable d'ouverts de Zariski non vides. Une fibre $Y_{t}$ de $h$ est dite générale si $t \in T$ est général. 
Rappelons aussi la notion d'orbifolde (géométrique lisse) spéciale :

Definition 7.11 Une orbifolde géométrique lisse $(Y \mid \Delta)$, avec $Y$ compacte et connexe est dite spéciale si :

1. $Y \in \mathcal{C}$ (ie : $Y$ est biméromorphe à une variété Kählérienne compacte).

2. Il n'existe pas de fibration $f:(Y \mid \Delta) \rightarrow X$ de type général. (De manière équivalente : $(Y \mid \Delta)$ n'a pas d'application méromorphe dominante "stable" sur une orbifolde géométrique de type général de dimension strictement positive. Cette notion se formule donc naturellement dans la catégorie biméromorphe des orbifoldes géométriques).

Exemple 7.12 Soit $(Y \mid \Delta):=\left(\mathbb{P}^{r} / D_{r}\right)$ l'orbifolde géométrique (logarithmique) lisse de l'exemple 2.20. Alors tous les faisceaux $S_{N, q}(Y \mid \Delta)$ sont triviaux. Il n'existe donc pas de fibration de type général $f:(Y \mid \Delta) \rightarrow X$ sur cette orbifolde géométrique, et $F T G(Y \mid \Delta)=0$ dans ce cas. Cette orbifolde géométrique est donc spéciale. Une seconde démonstration de cette propriété est donnée en 6.11 .

Les orbifoldes géométriques spéciales forment avec les orbifoldes géométriques de type général les deux classes antithétiques fondamentales de la classification biméromorphe des orbifoldes géométriques. Elles seront étudiées plus en détail dans la section 8 . Le résultat principal du présent texte est le scindage fonctoriel de toute orbifolde géométrique en ses parties spéciale (les fibres orbifoldes) et de type général (la base orbifolde) par une unique fibration (son coeur) dans la section 9.

De 7.3 on déduit :

Corollaire 7.13 Si $u:(X \mid \Delta) \rightarrow\left(X^{\prime} \mid \Delta^{\prime}\right)$ est méromorphe surjective entre orbifoldes géométriques lisses, et si $(X \mid \Delta)$ est spéciale, alors $\left(X^{\prime} \mid \Delta^{\prime}\right)$ est aussi spéciale.

De 7.8 on déduit le :

Corollaire 7.14 Soit $f:(Y \mid \Delta) \rightarrow X$ une application méromorphe surjective de type général, l'orbifolde géométrique $(Y \mid \Delta)$ étant lisse, $Y$ compacte et connexe. Supposons aussi qu'il existe une fibration méromorphe $h:(Y \mid \Delta) \rightarrow T$ telle que les fibres orbifoldes générales de $h$ soient spéciales. Alors $h$ se factorise par $f$ (ie : il existe $g: T \rightarrow X$ telle que $f=g \circ h$ ). 
Démonstration : Sinon, d'après le théorème 7.8 précédent, la restriction de $f$ à $\left(Y_{t} \mid \Delta_{Y_{t}}\right)$ est de type général, ce qui est impossible, puisque les fibres orbifoldes générales sont suposées spéciales

À l'aide de l'exemple 7.12, on obtient le résultat suivant, utilisé dans la $\S 7.4$ ci-dessous :

Corollaire 7.15 Soit $f:(Y \mid \Delta) \rightarrow X$ une application méromorphe surjective de type général, l'orbifolde géométrique $(Y \mid \Delta)$ étant lisse, $Y$ compacte et connexe. Supposons aussi qu'il existe une fibration holomorphe $h: Y \rightarrow T$ telle que les fibres génériques $Y_{t} \cong \mathbb{P}^{r}$ de $h$ soient des sous-variétés lisses connexes de $Y$ rencontrant transversalement le support de $\Delta$ en $s \leq(r+1)$ hyperplans projectifs. Alors la famille des fibres de $h$ se factorise par $f$.

\subsection{Presque-holomorphie des fibrations de type général}

Definition 7.16 Soit $f: Y \rightarrow X$ une application holomorphe propre et surjective (définition par passage au graphe $Y_{f} \subset X \times Y$ de $f$ ) entre espaces analytiques normaux connexes. Soit $I_{f} \subset Y$ son lieu d'indétermination (lieu au-dessus duquel le graphe de $f$ a des fibres de dimension strictement positive), et $f\left(I_{f}\right) \subset X$ l'image de $I_{f}$ par $f$ (image par $f^{\prime}: Y_{f} \rightarrow X$ de l'image réciproque de $I_{f}$ dans $\left.Y_{f}\right)$.

On dit que $f$ est presque-holomorphe si $f\left(I_{f}\right) \subsetneq X$.

L'exemple le plus simple de fibration méromorphe non presqueholomorphe est $f: \mathbb{P}^{2} \rightarrow \mathbb{P}^{1}$ dont les fibres sont les droites passant par un point $a \in \mathbb{P}^{2}$. La famille des fibres de cette fibration (vue dans $\mathcal{C}\left(\mathbb{P}^{2}\right)=$ Chow $\left(\mathbb{P}^{2}\right)$ ) se déforme (en fonction de $a$ ).

Par contraste, les applications presque-holomorphes jouissent de propriétés similaires à celles des applications holomorphes. Par exemple : si $f: Y \rightarrow X$ est une fibration presque-holomorphe, la famille des ses fibres (famille vue dans la variété de Chow $\mathcal{C}(Y)$ ) forme une composante irréductible de $\mathcal{C}(X)$, par le classique "lemme de rigidité".

En particulier, l'ensemble des fibrations presque-holomorphes (à équivalence biméromorphe près) sur une variété complexe fixée $Y$ (dénombrable à l'infini) est fini ou dénombrable. Remarquer que cette propriété tombe en défaut sur l'exemple non presque-holomorphe très simple donné ci-dessus. 
De plus, si $f$ est presque-holomorphe, la restriction $\left(\Delta_{Y}\right)_{x}$ de l'orbifolde géométrique $\Delta_{Y}$ à sa fibre générique $Y_{x}$ est bien définie, et $\left(Y_{x} /\left(\Delta_{Y}\right)_{x}\right)$ est lisse si $\left(Y \mid \Delta_{Y}\right)$ est lisse. La classe d'équivalence biméromorphe de $\left(Y_{x} /\left(\Delta_{Y}\right)_{x}\right)$ ne dépend que de celle de $\left(Y \mid \Delta_{Y}\right)$.

Théorème 7.17 Si $(Y \mid \Delta)$ est une orbifolde géométrique lisse, avec $Y$ compacte et connexe, et si $f:(Y \mid \Delta) \rightarrow X$ est une fibration méromorphe de type général, alors $f$ est presque-holomorphe.

Démonstration : Soit $v: Y^{\prime} \rightarrow Y$ une composée d'éclatements de centres lisses telle que $f^{\prime}:=f \circ v: Y^{\prime} \rightarrow X$ soit holomorphe. Si $f$ n'est pas presque holomorphe, l'un des diviseurs exceptionnels $E$ de l'un de ces éclatements est tel que :

1. $f^{\prime}(E)=X$,

2. $v(E):=T$ est de codimension $r+1 \geq 2$ dans $Y$.

3. Les fibres génériques $E_{t}$ de $E$ sur $T$ ont des images $X_{t}$ dans $X$ de dimension $d>0$. (Autrement dit $: f_{E}^{\prime}: E \rightarrow X$ ne se factorise pas par $v$ ).

4. On peut supposer que $E$ n'est pas contenu dans le support de $\Delta^{\prime}$ (ceci par le corollaire 4.15, puisque $f$, et donc $f^{\prime}$ sont de type général).

Puisque $(Y \mid \Delta)$ est lisse, $T$ est contenu dans $s \leq(r+1)$ des composantes (lisses, d'intersections transversales) du support de $\Delta$. Pour $t \in T$ générique, l'orbifolde géométrique $\left(E_{t} \mid \Delta_{E_{t}}\right)$ est donc biméromorphe à $\left(\mathbb{P}^{r} \mid \Delta_{r}\right)$, où $\Delta_{r}$ est supportée par $s$ hyperplans projectifs en position générale. Donc $\left(E_{t} \mid \Delta_{E_{t}}\right)$ est spéciale. Mais d'après 7.6, $f_{E}$ est de type général, donc d'après $7.8, f_{E_{t}}$ aussi. Mais ceci contredit 7.12, dont la démonstration est indépendante de ce qui précède.

\section{Remarque 7.18}

1. La condition de lissité de $(Y \mid \Delta)$ est essentielle. Soit $f:\left(\mathbb{P}^{2} \mid \Delta\right) \rightarrow-\rightarrow \mathbb{P}^{1}$ la fibration dont les fibres sont les droites passant par un point donné $a \in \mathbb{P}^{2}$, et soit $\Delta=(1-1 / m) \cdot\left(D_{1}+D_{2}+D_{3}\right)$, les $D_{j}$ étant 3 droites concourantes en $a \in \mathbb{P}^{2}$. Cette fibration est de type général si $m \geq 4$ (puisque sa base orbifolde géométrique l'est : considérer $u^{+}(\Delta)$ sur l'éclaté de $\mathbb{P}^{2}$ en a). Elle n'est évidemment pas presque-holomorphe.

2. Lorsque $\Delta=0$, la condition de lissité de $Y$ est également essentielle (Exemple 2.23, p. 534 de [Ca04] du cône $Y$ sur une variété de type général).

Ceci semble indiquer que la condition de lissité d'une orbifolde géométrique (par condition de croisements normaux sur le support de $\Delta$ ) est l'analogue adéquat du cas où $\Delta=0$. 


\subsection{Réduction de type général simultanée.}

Definition 7.19 Soit $g:(Y \mid \Delta) \rightarrow X$ une fibration de type général, avec $(Y \mid \Delta)$ lisse, $Y$ compacte et connexe. On dit que $g$ est maximum si toute autre fibration de type général $h:(Y \mid \Delta) \rightarrow V$ est dominée par $g$ (ie : telle qu'existe $k: X \rightarrow V$ avec $h=k \circ g$ ).

Une telle fibration de type général maximum est unique (à équivalence biméromorphe près) si elle existe. L'existence (qui est l'un des résultats principaux du présent texte) sera établie pour les variétés de la classe $\mathcal{C}$ dans la section 9, à l'aide du théorème d'additivité orbifolde pour les dimensions canoniques. On utilisera en particulier le :

Théorème 7.20 Soit $f:(Y \mid \Delta) \rightarrow S$ une application holomorphe surjective, avec $(Y \mid \Delta)$ une orbifolde géométrique lisse, $Y$ étant compacte connexe et dans la classe $\mathcal{C}$. On suppose qu'il existe $T \subset S$, un sous-ensemble (arbitraire $^{27}$ ) non contenu dans une réunion dénombrable de fermés de Zariski stricts $^{28}$ de $S$, tel que pour tout $t \in T$, l'orbifolde géométrique (que l'on peut supposer lisse) $\left(Y_{t} \mid \Delta_{t}\right):=\left(Y_{t} \mid \Delta_{Y_{t}}\right)$ admette une fibration de type général $\overline{g_{t}}:\left(Y_{t} \mid \Delta_{t}\right) \rightarrow X_{t}$ maximum.

Il existe alors une unique fibration $g:(Y \mid \Delta) \rightarrow X$ au-dessus de $f$ (ie : telle qu'existe $h: X \rightarrow S$ avec $f=h \circ g$ ) telle que pour $x \in U$ général dans $S, g_{s}:\left(Y_{s} \mid \Delta_{s}\right) \rightarrow X_{s}$ soit la fibration de type général maximum de $\left(Y_{s} \mid \Delta_{s}\right)$. De plus, pour tout $s=t \in T \cap U, g_{s}=\bar{g}_{t}$

Démonstration : Le théorème 2.35, p. 538 de [Ca04] montre l'existence d'une fibration $g:(Y \mid \Delta) \rightarrow X$ au-dessus de $S$ telle que $g_{t}=\bar{g}_{t}$ pour tout $t \in T^{\prime} \subset T, T^{\prime}$ n'étant pas analytiquement maigre dans $S$. (Les arguments de la démonstration de [Ca04,thm 2.35] s'appliquent sans changement à la situation considérée ici, et plus généralement au cas de fibrations presqueholomorphes possédant une propriété d'unicité sur les fibres $Y_{t}, t \in T$ de $f)$.

Le théorème résulte alors de la proposition suivante :

Proposition 7.21 Soit $f:(Y \mid \Delta) \rightarrow S$ une application holomorphe surjective, avec $(Y \mid \Delta)$ une orbifolde géométrique lisse, $Y$ étant compacte connexe.

\footnotetext{
27 ie : non nécessairement analytique.

${ }^{28}$ ie : différents de $S$. Un tel ensemble $T$ est dit ne pas être analytiquement maigre dans $S$.
} 
Soit $f=h \circ g$ une factorisation de $f$ par une application méromorphe $g: Y \rightarrow X$ et une fibration $h: X \rightarrow S$.

On suppose que la restriction $g_{t}:\left(Y_{t} \mid \Delta_{t}\right) \rightarrow X_{t}$ est de type général pour tout $t \in T, T$ non analytiquement maigre dans $S$. Alors, $g_{s}$ est de type général pour $s \in S$ général.

Démonstration : Pour tout entier $m>0$, considérons le faisceau analytique cohérent $L:=g^{*}\left(K_{X / S}\right) \subset \Omega_{Y / S}^{p}$, avec $p:=\operatorname{dim}(X / S)=$ $\operatorname{dim}(X)-\operatorname{dim}(S)$, et la saturation $\overline{L_{m}} \subset S_{m, p}((Y \mid \Delta) / S)$ de $L^{\otimes m}$ dans le faisceau $S_{m, p}\left((Y \mid \Delta) / S\right.$ ) quotient de $S_{m, p}(Y \mid \Delta)$ par le sous-faisceau des (polynômes en les) formes nulles sur les $p$-vecteurs tangents à $Y$ qui sont $f$ verticaux.

Par le théorème d'image directe de Grauert, les faisceaux $F_{m}:=f_{*}\left(\overline{L_{m}}\right)$ sont cohérents, et, pour $s \in U$ général dans $S$, la fibre $F_{m, s}$ de $F_{m}$ en $s$ coincide avec $H^{0}\left(Y_{s},\left(\overline{L_{f_{s}}}\right)_{m}\right)$ pour tous les $m>0$. On a noté $L_{g_{s}}:=\left(g_{s}\right)^{*}\left(K_{X_{s}}\right) \subset \Omega_{Y_{s}}^{p}$, et $\overline{L_{g_{s}}, m} \subset S_{m, p}\left(Y_{s} \mid \Delta_{s}\right)$ la saturation de $L_{g_{s}}^{\otimes m}$ dans $S_{m, p}\left(Y_{s} \mid \Delta_{s}\right)$.

L'assertion résulte alors de ce que si, pour un $s \in U$, le système linéaire $\left|\overline{L_{g_{s}}, m}\right|$ définit une application $\varphi_{s}: Y_{s} \rightarrow Z_{s}$ de rang $p$, il en est de même pour tout $s^{\prime} \in S$ générique, et qu'alors $Z_{s}=X_{s}$ (quitte à remplacer $m$ par un multiple adéquat). 


\section{ORBIFOLDES SPÉCIALES}

\section{GÉOMÉTRIQUES}

\subsection{Fibre et base orbifolde d'une fibration}

Rappels. Dans cette section, on considèrera uniquement des orbifoldes géométriques $(X \mid \Delta)$ lisses, avec $X \in \mathcal{C}$, lisse et connexe.

Si $f:(X \mid \Delta) \rightarrow Y$ est méromorphe surjective, on définira sa base orbifolde stable, notée $[Y \mid \Delta(f, \Delta)]$ ou $[f \mid \Delta]$, obtenue comme base orbifolde d'un représentant holomorphe net arbitraire de $f$. Nous ne savons pas si la classe d'équivalence biméromorphe (au sens orbifolde) de $[Y \mid \Delta(f, \Delta)]=[f \mid \Delta]$ est indépendante du modèle choisi. Mais, par 4.14, la dimension canonique $\kappa([f \mid \Delta])$ est bien définie. C'est donc sur cet unique invariant que sont basées toutes les considérations qui suivent.

On dit (definition 4.17) que $f$ est de type général si sa base orbifolde stable est de type général et de dimension strictement positive.

Si $f:(X \mid \Delta) \rightarrow Y$ est une fibration, on désignera par $(X \mid \Delta)_{y}$ sa fibre orbifolde générique stable, définie, par modification de $X$, sur un modèle holomorphe de $f$ (également noté $f$ ), dont la fibre orbifolde générique $\left(X_{y} \mid \Delta_{y}\right)$ est alors lisse (par le théorème de Sard). Remarquons que la classe d'équivalence biméromorphe de $(X \mid \Delta)_{y}$ générique dépend en général du modèle biméromorphe de $f$ choisi, rendant $f$ holomorphe. Voir cependant 7.16 et 7.17 ci-dessous : si $f$ est presque-holomorphe (définition 7.16 ), alors $(X \mid \Delta)_{y}$ ne dépend pas du modèle choisi, et $f$ est presque-holomorphe si elle est de type général.

Lorsque $f$ est holomorphe, on a donc : $(X \mid \Delta)_{y}=\left(X_{y} \mid \Delta_{y}\right), \Delta_{y}$ étant la restriction (transversale) de $\Delta$ à $X_{y}$.

- Rappelons aussi que, par 7.14, si $f:(X \mid \Delta) \rightarrow Y$ est une fibration de type général, et si $g:(X \mid \Delta) \rightarrow T$ est une fibration dont la fibre générique (orbifolde stable) $(X \mid \Delta)_{t}$ est spéciale, il existe une (unique) factorisation $h: T \rightarrow Y$ de $f$ telle que $f=h \circ g$.

- Rappelons enfin (4.18) la :

Definition 8.1 L'orbifolde géométrique lisse $(X \mid \Delta)$ est dite spéciale si :

1. $X \in \mathcal{C}$. 
2. Aucune fibration $f:(X \mid \Delta) \rightarrow Y$ n'est de type général.

(En particulier, $X \in \mathcal{C}$ est dite spéciale si $(X \mid 0)$ est spéciale).

\subsection{Premiers exemples}

Les exemples et contre-exemples les plus simples sont les suivants :

\section{Exemple 8.2}

1. Si $(X \mid \Delta)$ est lisse de type général, de dimension non nulle, alors $(X \mid \Delta)$ n'est pas spéciale.

2. Si $X$ est une courbe lisse projective de genre $g$, et si $\Delta=\sum_{j}\left(1-\frac{1}{m_{j}}\right) \cdot p_{j}$, avec $m_{j}>1, \forall j \in J$, les $p_{j}$ étant des points distincts de $X$, alors $(X \mid \Delta)$ est spéciale si et seulement si $2(g-1)+\sum_{j}\left(1-1 / m_{j}\right) \leq 0$.

C'est le cas si et seulement si $g=1$ et $\Delta=0$, ou $g=0$, et si la suite ordonnée croissante des $m_{j}, j \in J$ est l'une des suivantes :

$|J| \leq 2$ : quelconque.

$|J|=3:(2,2, m), \forall m \leq+\infty ;(2,3,4) ;(2,3,5) ;(2,3,6) ;(2,4,4)$.

$|J|=4:(2,2,2,2)$.

3. Si $f:(X \mid \Delta) \rightarrow\left(X^{\prime} \mid \Delta^{\prime}\right)$ est méromorphe surjective (ie : telle que $f$ ait un modèle biméromorphe lisse qui est un morphisme orbifolde sujectif), et si $(X \mid \Delta)$ est spéciale, alors $\left(X^{\prime} \mid \Delta^{\prime}\right)$ est spéciale, par 7.3.

4. Si $(X \mid \Delta)$ est une orbifolde géométrique lisse, si $X \in \mathcal{C}$, et si $\kappa(X \mid \Delta)=$ 0 , alors $(X \mid \Delta)$ est spéciale. (Ceci résulte du théorème 6.7).

5. Si $(X \mid \Delta)$ est lisse et Fano, alors $(X \mid \Delta)$ est spéciale (par le corollaire 6.10). Plus généralement (du moins conjecturalement) :

6. Si $\kappa_{+}(X \mid \Delta)=-\infty$ (voir définition 5.46), et en particulier si $(X \mid \Delta)$ est $R E$ (voir définition 5.19), alors $(X \mid \Delta)$ est spéciale. C'est évident, par définition.

7. Si $(X \mid \Delta)$ est $\mathcal{S}$-connexe (voir définition en 9.7), alors $(X \mid \Delta)$ est spéciale (par le corollaire 9.7 du chapitre suivant).

8. Pour tout $n \geq 0$ et tout $k \in\{-\infty, 0,1, \ldots, n-1\}$, il existe des orbifoldes géométriques spéciales de dimension $n$ avec $\kappa=k$ (Voir [Ca04] pour des exemples simples).

9. Si $X$ est une variété quasi-projective lisse, et $\bar{X}$ une compactification lisse de $X$ telle que $\bar{X}-X:=D$ soit un diviseur à croisements normaux de $\bar{X}$, on dira que $X$ est spéciale si et seulement si $(\bar{X} \mid D)$ est spéciale. Cette condition ne dépend pas de la compactification choisie. 
10. $X$ est spéciale s'il existe une application méromorphe non-dégénérée $\varphi: \mathbb{C}^{n} \rightarrow X$ (voir [Ca 04], qui traite d'une situation plus générale). De manière similaire, s'il existe un morphisme orbifolde $\varphi: \mathbb{C}^{n} \rightarrow(X \mid \Delta)$, alors $(X \mid \Delta)$ n'est pas de type général ([Sak 74]. Je remercie E. Rousseau pour m'avoir indiqué cette référence).

\subsection{Composition de fibrations spéciales}

Le résultat simple suivant est crucial :

Théorème 8.3 Soit $f:(X \mid \Delta) \rightarrow Y$ une fibration méromorphe surjective telle que :

1. $X \in \mathcal{C}$

2. La base orbifolde stable $[Y \mid \Delta(f, \Delta)]$ de $f$ est spéciale.

3. La fibre générale orbifolde stable $(X \mid \Delta)_{y}$ de $f$ est spéciale.

Alors : $(X \mid \Delta)$ est spéciale.

Démonstration : On supposer que $f$ est nette, à base orbifolde lisse spéciale, et à fibres orbifoldes générales spéciales. Soit $g:(X \mid \Delta) \rightarrow T$ une fibration de type général. Il résulte de 7.14 que $g=h \circ f$ pour une fibration $h: Y \rightarrow T$. Quitte à changer de modèles biméromorphes pour $f, g, h$, on peut supposer par 3.6 que $\Delta_{T}(g, \Delta)=\Delta_{T}\left(h, \Delta_{Y}(f, \Delta)\right)$ est la base orbifolde stable de $h:(Y \mid \Delta(f, \Delta)) \rightarrow T$. Puisque $g$ est de type général, il en est donc de même pour $h$. Mais ceci contredit le fait que $[(Y \mid \Delta(f, \Delta)]$ est spéciale. Donc $(X \mid \Delta)$ est spéciale.

Remarque 8.4 Ce résultat est évidemment faux (surfaces elliptiques de base $\mathbb{P}^{1}$ ayant au moins 5 fibres multiples, par exemple) sans l'introduction de structures orbifoldes géométriques. Ce résultat simple est l'un de ceux qui justifient la nécessité de travailler dans la catégorie des orbifoldes géométriques, plutôt que dans celle des variétés. Un autre résultat similaire est celui portant sur les groupes fondamentaux des fibrations : celui de l'espace total est extension de celui de la base orbifolde par (un quotient de) celui de la fibre (orbifolde), ceci sous des conditions faibles.

Par itération, on obtient, à l'aide de 3.15 :

Corollaire 8.5 Soit $f_{j}:\left(X_{j} \mid \Delta_{j}\right) \rightarrow X_{j+1}, j=0, \ldots, k-1$, une suite de fibrations méromorphes telle que: 
1. $X_{0} \in \mathcal{C}$.

2. $X_{k}$ est un point.

3. Pour tout $j=0, \ldots, k-1,\left[\left(X_{j+1} \mid \Delta_{j+1}\right)\right]=\left[\left(X_{j+1} \mid \Delta\left(f_{j}, \Delta_{j}\right)\right]\right.$.

4. Pour tout $j=0, \ldots, k-1$, la fibre orbifolde stable générique $F_{j}$ de $f_{j}$ est telle que : ou bien $\kappa\left(F_{j}\right)=0$, ou bien $\kappa_{+}\left(F_{j}\right)=-\infty$.

Alors : $\left(X_{0} \mid \Delta_{0}\right)$ est spéciale.

Nous verrons au $\S 10$ que, réciproquement, sous réserve de la validité de la conjecture $C_{n, m}^{o r b}$, on peut canoniquement décomposer les orbifoldes géométriques spéciales en tours de fibrations à fibres orbifoldes génériques ayant soit $\kappa=0$, soit $\kappa_{+}=-\infty$. 


\section{LE "COEUR" D'UNE ORBIFOLDE GÉOMÉTRIQUE}

\subsection{Construction du Coeur.}

Le résultat central du présent texte est le :

Théorème 9.1 Soit $(Y \mid \Delta)$ une orbifolde géométrique lisse, avec $Y$ compacte connexe et dans la classe $\mathcal{C}^{29}$ Il existe alors une unique ${ }^{30}$ fibration $c_{(Y \mid \Delta)}$ : $(Y \mid \Delta) \rightarrow C(Y \mid \Delta)$ telle que :

1. $\kappa\left(c_{(Y \mid \Delta)} \mid \Delta\right)=\operatorname{dim}(C(Y \mid \Delta))$ (et c est donc presque-holomorphe).

2. Les fibres générales $\left(Y_{c} \mid \Delta_{c}\right)$ de $c_{(Y \mid \Delta)}$ sont spéciales.

La fibration $c_{(Y \mid \Delta)}$ est appelée le coeur ${ }^{31}$ de $(Y \mid \Delta)$.

On notera $[C(Y \mid \Delta)]$ la base orbifolde stable (voir 4.14) de $c_{(Y \mid \Delta)}{ }^{32}$.

La dimension de $C(X \mid \Delta)$ est appelée dimension essentielle de $(X \mid \Delta)$, et est notée ess $(X \mid \Delta)$.

On notera aussi $K(C(Y \mid \Delta)):=A(Y \mid \Delta)$ l'algèbre canonique (voir définition en 2.16) de la base orbifolde stable de $c_{(Y \mid \Delta)}$. On l'appelle l'algèbre essentielle de $(Y \mid \Delta)$.

\section{Remarque 9.2}

(1) La fibration $c_{(Y \mid \Delta)}$ est donc de type général si $\operatorname{dim}(C(Y \mid \Delta))>0$. Sinon, $\operatorname{dim}(C(Y \mid \Delta))=0$ si et seulement si $(Y \mid \Delta)$ est spéciale.

(2) A l'autre extrême, $(Y \mid \Delta)$ est de type général si et seulement si $\operatorname{dim}(C(Y \mid \Delta))=\operatorname{dim}(Y)$, c'est-à-dire si et seulement si $c_{(Y \mid \Delta)}=i d_{Y}$.

(3) D'après 7.14, le coeur est donc l'unique fibration de type général maximum (au sens de 7.19) sur $(Y \mid \Delta)$ si $(Y \mid \Delta)$ n'est pas spéciale.

(4) Si $C_{n, m}^{o r b}$ est vraie, alors le coeur défini en 9.1 coincide avec celui défini en 10.2.

Démonstration : L'unicité est une conséquence immédiate du théorème 7.14. On montre donc maintenant l'existence. On procède par récurrence sur

\footnotetext{
${ }^{29}$ ie : biméromorphe à $Y^{\prime}$, Kählérienne compacte.

${ }^{30} \mathrm{~A}$ équivalence biméromorphe près

${ }^{31}$ Ce terme est choisi pour insister sur le fait que cette fibration décrit la structure de $X$ dans son aspect le plus essentiel.

${ }^{32}$ Nous ne savons pas si c'est un invariant de $c_{(Y \mid \Delta)}$ dans la catégorie biméromorphe des orbifoldes géométriques lisses.
} 
$n:=\operatorname{dim}(Y)$. Si $n=0$, la conclusion est (évidemment) vraie, en considérant les points comme des orbifoldes géométriques spéciales. Supposant la conclusion vraie pour $\operatorname{dim}\left(Y^{\prime}\right)<n$, on choisit pour $c_{(Y \mid \Delta)}$ une application constante si $(Y \mid \Delta)$ est spéciale. Sinon, on choisit une fibration $f:(Y \mid \Delta) \rightarrow S$ de type général, avec $p:=m(X)>0$ maximum. Il suffit de montrer que les fibres générales (orbifoldes) de $f$ sont spéciales : on aura alors $f=c_{(Y \mid \Delta)}$. Si les fibres orbifoldes générales de $f$ ne sont pas spéciales, il existe un sousensemble $T \subset S$ qui n'est pas analytiquement maigre dans $S$, et tel que pour $s \in T,\left(Y_{s} \mid \Delta_{s}\right)$ n'est pas spéciale, et $c_{\left(Y_{s} \mid \Delta_{s}\right)}$ est donc, par l'hypothèse de récurrence, puique $\operatorname{dim}(S)>0$, par hypothèse, l'unique fibration de type général maximum sur $\left(Y_{s} \mid \Delta_{s}\right)$. On déduit alors de 7.20 et 7.21 l'existence d'une factorisation $f=h \circ g$ de $f$, avec $g: Y \rightarrow X$ et $h: X \rightarrow S$ des fibrations, telles que $\operatorname{dim}(X)>\operatorname{dim}(S)$, et telle que, pour $s \in S$ général, la restriction $g_{s}:\left(Y_{s} \mid \Delta_{s}\right) \rightarrow X_{s}$ de $g$ à $Y_{s}$ soit de type général.

Puisque $f=h \circ g$ est de type général, on déduit du théorème 6.3 que $g:(Y \mid \Delta) \rightarrow X$ est une fibration de type général. Ceci contredit le fait que $\operatorname{dim}(S)$ soit maximum parmi les bases de fibrations de type général définies sur $(Y \mid \Delta)$, et montre donc que les fibres orbifoldes de $f$ sont spéciales.

Exemple 9.3 Soit $(Y \mid \Delta)$ une orbifolde géométrique lisse avec $Y$ compacte et connexe dans la classe $\mathcal{C}$. Si $\kappa(Y \mid \Delta) \geq 0$, alors $\operatorname{dim}(C(Y \mid \Delta)) \leq \kappa(Y \mid \Delta)$, et on a égalité si et seulement si $c_{(Y \mid \Delta)}=M_{(Y \mid \Delta)}$ est la fibration de MoishezonIitaka de l'orbifolde géométrique $(Y \mid \Delta)$. Ceci résulte imédiatemment du théorème 6.8.

Proposition 9.4 Soit $(Y \mid \Delta)$ une orbifolde géométrique lisse, avec $Y \in \mathcal{C}$. Soit $f:(Y \mid \Delta) \rightarrow S$ une fibration méromorphe à fibres orbifoldes générales spéciales, et $g:(Y \mid \Delta) \rightarrow X$ une fibration méromorphe telle que $\kappa(g \mid \Delta)=$ $\operatorname{dim}(X)$. On pose $c:=c_{(Y \mid \Delta)}$.

Il existe alors une unique couple de fibrations $\sigma: S \rightarrow C(Y \mid \Delta)$ et $\gamma$ : $C(Y \mid \Delta) \rightarrow X$ telles que : $c \circ \sigma=f$ et $\gamma \circ c=g$.

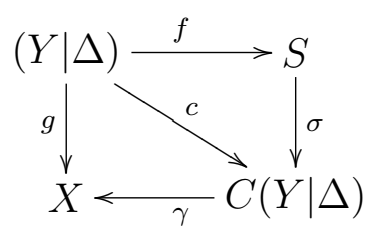

Démonstration : C'est une conséquence immédiate de 7.14 : pour construire $\sigma$ (resp. $\gamma$ ), on utilise le fait que $c$ est à base orbifolde stable 
de type général (resp. à fibre orbifolde générale spéciale).

\subsection{Fonctorialité.}

Proposition 9.5 Soit $f:(Y \mid \Delta) \rightarrow\left(X \mid \Delta^{\prime}\right)$ une application méromorphe surjective d'orbifoldes géométriques lisses, avec $Y \in \mathcal{C}$. Alors $f$ induit une (unique) application méromorphe d'orbifoldes géométriques lisses $c_{f}$ : $C(Y \mid \Delta) \rightarrow C\left(X \mid \Delta^{\prime}\right)$ telle que $c_{\left(X \mid \Delta^{\prime}\right)} \circ f=c_{f} \circ c_{(Y \mid \Delta)}$.

De plus, $f$ induit une application méromorphe orbifolde $c_{f}:[C(Y \mid \Delta)] \rightarrow$ $\left[C\left(X \mid \Delta^{\prime}\right)\right]$ entre bases orbifoldes stables.

Démonstration : La composée $c_{\left(X \mid \Delta^{\prime}\right)} \circ f:(Y \mid \Delta) \rightarrow C\left(X \mid \Delta^{\prime}\right)$ est de type général, par la proposition 7.3. Sa restriction à la fibre générale orbifolde de $c_{(Y \mid \Delta)}$ est donc, par le théorème 7.6, soit constante, soit de type général. Comme cette fibre orbifolde est spéciale, cette application est constante, et $c_{\left(X \mid \Delta^{\prime}\right)} \circ f$ se factorise donc par $c_{(Y \mid \Delta)}$. La seconde assertion résulte de 3.6, en considérant des représentants strictement nets et hauts.

\subsection{Connexité par chaînes spéciales.}

Proposition 9.6 Soit $(Y \mid \Delta)$ une orbifolde géométrique lisse, avec $Y \in \mathcal{C}$, et $\left.c_{(Y \mid \Delta)}:(Y \mid \Delta) \rightarrow C(Y \mid \Delta)\right)$ son coeur. Si $\left(V_{t}\right)_{t \in T}$ est une famille couvrante de sous-variétés de $Y$, et si $\left(V_{t} \mid \Delta_{V_{t}}\right)$ (définie par restriction de $\Delta$ à $V_{t}$ comme en 2.38) est spéciale pour $t \in T$ général, alors $V_{t}$ est contenue dans la fibre de $c_{(Y \mid \Delta)}$, pour tout $t \in T$.

Démonstration : Sinon, la restriction de $c_{(Y \mid \Delta)}$ est de type général, par le théorème 7.8 , ce qui contredit le fait que $\left(V_{t} \mid \Delta_{V_{t}}\right)$ est spéciale.

Corollaire 9.7 Soit $(Y \mid \Delta)$ une orbifolde géométrique lisse, $Y \in C$ compacte et connexe. Supposons que deux points généraux de $Y$ peuvent être joints par une chaine connexe de sous-variétés (au sens de 2.38) de $Y$ telles que les sous-orbifoldes géométriques de $(Y \mid \Delta)$ obtenues par restriction à ces sousvariétés (au sens de 2.38) soient spéciales (On dira alors que $(Y \mid \Delta)$ est $\mathcal{S}$-connexe).

Alors $(Y \mid \Delta)$ est spéciale. (Autrement dit : $(Y \mid \Delta)$ est spéciale si elle est lisse et $\mathcal{S}$-connexe). 
Démonstration : Soit $y$ l'un des deux points, choisi tel que la fibre $F$ de $c:=c_{(Y \mid \Delta)}$ passant par $y$ ne passe pas par le lieu d'indétermination de $c_{(Y \mid \Delta)}$. Ceci est possible, puisque $c_{(Y \mid \Delta)}$ est presque-holomorphe, en vertu du théorème 7.4. Soit $V \subset Y$ une sous-variété telle que $\left(V \mid \Delta_{V}\right)$ soit spéciale et rencontre $F$ sans être contenue dans $F$ (on peut supposer que la restriction de $\Delta$ à $V$ est définie, par le même théorème 7.8 et la remarque 7.9). Alors $c(V) \neq$ $C(Y \mid \Delta$ ), par 7.6. Par récurrence sur $\operatorname{dim}(Y)$, on en déduit (en considérant les variétés de la forme $\left.W:=c^{-1}(V)\right)$ que $(Y \mid \Delta)$ admet une famille couvrante de sous-orbifoldes géométriques $\left(W \mid \Delta_{W}\right)$ contenant strictement les fibres de c. Contradiction avec 9.6.

Remarque 9.8 La lissité de $(Y \mid \Delta)$ est essentielle (considérer le cône sur une variété de type général). En sens inverse, il existe des $Y$ (avec $\Delta=$ 0) spéciales n'ayant aucune famille couvrante non-triviale de sous-variétés spéciales : par exemple, les variétés abéliennes simples.

Corollaire 9.9 Si $(Y \mid \Delta)$ est lisse, avec $Y \in \mathcal{C}$, et si $(Y \mid \Delta)$ est rationnellement connexe par chaines, alors $(Y \mid \Delta)$ est spéciale.

\subsection{Invariance par revêtement étale.}

Proposition 9.10 Soit $v:\left(Y^{\prime} \mid \Delta^{\prime}\right) \rightarrow(Y \mid \Delta)$ un morphisme d'orbifoldes géométriques lisses et connexes dans la classe $\mathcal{C}$. Si v est étale en codimension 1 (au sens orbifolde, voir 2.21 et 2.22), alors $c_{v}: C\left(Y^{\prime} \mid \Delta^{\prime}\right) \rightarrow C(Y \mid \Delta)$ est génériquement fini.

Démonstration : On peut supposer que le revêtement $v$ est Galoisien de groupe fini $G$ en codimension 1 (ie : sur le complémentaire de $A$, Zariski fermé de codimension au moins 2 dans $Y$ ). On peut ensuite, quitte à augmenter $\Delta^{\prime}$ (ie : à remplacer $\Delta^{\prime}$ par $\Delta^{\prime+} \geq \Delta^{\prime}$ ), supposer que $\Delta^{\prime}$ est $G$-invariant : il suffit pour cela d'affecter chaque composante $D^{\prime}$ de $v^{-1}(\lceil\Delta\rceil)$ de la plus grande des multiplicités apparaissant initialement sur l'une des composantes de $v^{-1}\left(v\left(D^{\prime}\right)\right)$. Il nous suffit donc d'établir l'assertion lorsque $v:\left(Y^{\prime} \mid \Delta^{\prime}\right) \rightarrow$ $(Y \mid \Delta)$ est Galoisien en codimension 1 au sens précédent.

Le groupe $G$ agit naturellement sur $C\left(Y^{\prime} \mid \Delta^{\prime}\right)$, par unicité du coeur. Soit alors $q: C\left(Y^{\prime} \mid \Delta^{\prime}\right) \rightarrow C^{\prime}(Y \mid \Delta)$ le quotient par $G$ de $C\left(Y^{\prime} \mid \Delta^{\prime}\right)$. On a une application naturelle $c^{\prime}: Y \rightarrow C^{\prime}(Y \mid \Delta)$ telle que $c^{\prime} \circ v=q \circ c_{\left(Y^{\prime} \mid \Delta^{\prime}\right)}$. Les fibres orbifoldes de cette application sont spéciales, puisque images par $v$ de 
celles de $c_{\left(Y^{\prime} \mid \Delta^{\prime}\right)}$. De plus, $c^{\prime}$ est une fibration de type général, par 4.12. Donc $C^{\prime}(Y \mid \Delta)=C(Y \mid \Delta)$ et $c^{\prime}=c_{(Y \mid \Delta)}$. D'où le résultat. 


\section{DÉCOMPOSITION DU COEUR.}

La conjecture $C_{n, m}^{\text {orb }}$ implique que le "coeur" d'une orbifolde géométrique lisse $(Y \mid \Delta)$ de la classe $\mathcal{C}$ n'est autre que la composée des itérés, dans le cadre orbifolde géométrique (c'est essentiel), des deux fibrations de base de la classification : la fibration $M$ de Moishezon-Iitaka, et le quotient $\kappa$-rationnel $r$. En bref : $c=(M \circ r)^{n}, n=\operatorname{dim}(Y)$.

\subsection{La décomposition conditionnelle du coeur.}

On rappelle les propriétés de $M$ et $r$.

Lemme 10.1 On admet l'existence du $\kappa$-quotient rationnel ${ }^{33}$ pour les orbifoldes géométriques lisses dans $\mathcal{C}$.

Soit $(Y \mid \Delta)$ une orbifolde géométrique lisse, avec $Y \in \mathcal{C}$.

Soit $r: Y \rightarrow R^{+}=R^{+}(Y \mid \Delta)$ son $\kappa$-quotient rationnel, de base orbifolde stable $\left[R^{+} \mid \Delta_{R}^{+}\right]:=\left(R^{+} \mid \Delta(r, \Delta)\right)$. (Voir la $\left.\S 6.4\right)$.

Soit $M:\left(R^{+} \mid \Delta_{R}^{+}\right) \rightarrow M\left(R^{+}\right)$la fibration de Moishezon-Iitaka de $\left(R^{+} \mid \Delta_{R}^{+}\right)$(qui est bien définie, puisque $\kappa\left(R^{+} \mid \Delta_{R}^{+}\right) \geq 0$ ).

On notera $s_{(Y \mid \Delta)}:=M \circ r:(Y \mid \Delta) \rightarrow S(Y \mid \Delta)$ la composée, appelée réduction spéciale élémentaire $d e(Y \mid \Delta)$.

Alors :

1. Les fibres orbifoldes stables générales de $s_{(Y \mid \Delta)}$ sont spéciales.

2. $S(Y \mid \Delta)=(Y \mid \Delta)$ si et seulement si $(Y \mid \Delta)$ est de type général (ie : si et seulement si $\kappa(Y \mid \Delta)=\operatorname{dim}(Y) \geq 0)$.

Démonstration : La première assertion résulte de 8.3, la seconde de la remarque 6.15 précédente.

Théorème 10.2 On admet l'existence du $\kappa$-quotient rationnel pour les orbifoldes géométriques lisses dans $\mathcal{C}$, conséquence de $C_{n, m}^{\text {orb }}$.

Soit $(Y \mid \Delta)$ une orbifolde géométrique lisse, avec $Y \in \mathcal{C}$.

On définit une suite de fibrations $s^{k}:(Y \mid \Delta) \rightarrow S^{k}(Y \mid \Delta):=S^{k}$, de base orbifolde stable notée $\left(S^{k} \mid \Delta_{S^{k}}\right)$, par récurrence sur $k \geq 0$ par les conditions :

1. $s^{0}=i d_{Y}$.

2. $s^{k+1}:=s_{\left(S^{k} \mid \Delta_{S k}\right)}:\left(S^{k} \mid \Delta_{S^{k}}\right)-\rightarrow S^{k+1}$

Alors :

\footnotetext{
${ }^{33}$ Conséquence de $C_{n, m}^{\text {orb }}$.
} 
1. Il existe un plus petit entier $k \leq n=\operatorname{dim}(Y)$ tel que $S^{k+1}=S^{k}$.

Cet entier sera appelé la longueur de $(Y \mid \Delta)$, noté $\nu(Y \mid \Delta)$.

2. Si $k \geq \nu(Y \mid \Delta), s^{k}=s^{n}:(Y \mid \Delta) \rightarrow S^{k}=S^{n}$ est l'unique fibration de type général à fibres orbifoldes spéciales définie sur l'orbifolde géométrique $(Y \mid \Delta)$

La fibration $S^{n}$ (conditionnelle en $C_{n, m}^{\text {orb }}$ ) est donc le coeur de $(Y \mid \Delta)$. Elle a été construite inconditionnellement dans le $\S 9$.

Démonstration : La première assertion résulte de ce que $\operatorname{dim}\left(S^{k+1}\right)<$ $\operatorname{dim}\left(S^{k}\right)$ si $S^{k+1} \neq S^{k}$, et de ce que $S^{k+j}=S^{k}$ pour tout $j>0$ si cette égalité a lieu pour $j=1$. La seconde assertion résulte du lemme précédent, l'unicité de 8.3

Reformulons le résultat précédent :

Théorème 10.3 On admet l'existence du $\kappa$-quotient rationnel (conditionnel) pour les orbifoldes géométriques lisses dans $\mathcal{C}$. Soit $(Y \mid \Delta)$ une orbifolde géométrique lisse, avec $Y \in \mathcal{C}$ et $n=\operatorname{dim}(Y)$. Alors $: c=(M \circ r)^{n}$, avec les notations précédentes.

Pour les orbifoldes géométriques spéciales, on a donc :

Corollaire 10.4 On admet l'existence du $\kappa$-quotient rationnel (conditionnel) pour les orbifoldes géométriques lisses dans $\mathcal{C}$.

Soit $(Y \mid \Delta)$ une orbifolde géométrique lisse, avec $Y \in \mathcal{C}$, et $n=\operatorname{dim}(Y)$.

Alors $(Y \mid \Delta)$ est spéciale si et seulement si $\operatorname{dim}\left(S^{n}(Y \mid \Delta)\right)=0$.

De manière équivalente : $(Y \mid \Delta)$ est spéciale si et seulement si $(Y \mid \Delta)$ est (comme en 8.5) une tour de fibrations à fibres orbifoldes générales stables $F$ ayant soit $\kappa=0$, soit $\kappa_{+}=-\infty$.

Remarque 10.5 La suite de fibrations $s^{k}$ fournit une décomposition intrinsèque et fonctorielle dans la catégorie biméromorphe des orbifoldes géométriques lisses de $\mathcal{C}$ en composantes des trois "géométries pures" élémentaires :

1. $\kappa_{+}=-\infty$ (conjecturalement rationnellement connexes dans un sens orbifolde adéquat).

2. $\kappa=0$.

3. $\kappa=\operatorname{dim}$ ("type général").

Les analogues "numériques" de ces trois géométries biméromorphes sont: 
1. $K<0$ ("géométrie de Fano)

2. $K \equiv 0$. (ou $\left.c_{1}=0\right)$.

3. $K>0$ (fibré canonique ample).

L'objectif du programme des modèles minimaux (en version "logarithmique") est précisément de réduire la version biméromorphe à la version numérique. Ce "programme" pourrait être (en l'absence de méthodes biméromorphes directes) l'outil permettant de démontrer certaines des conjectures énoncées ci-dessous concernant les orbifoldes géométriques.

La "géométrie spéciale" combine donc (conditionnellement, et seulement dans la catégorie orbifolde biméromorphe) les deux premières "géométries pures" $\left(\kappa_{+}=-\infty\right.$ et $\left.\kappa=0\right)$, tandis que le "coeur" décompose canoniquement et fonctoriellement les orbifoldes géométriques dans $\mathcal{C}$ en leurs composantes "spéciale" (les fibres), et de "type général" (la base orbifolde).

\subsection{Existence de variétés de type et dimension donnés.}

Definition 10.6 Admettons la conjecture $C_{n, m}^{\text {orb }}$. Pour toute orbifolde géométrique $(X \mid \Delta)$ de dimension $n$, avec $X \in \mathcal{C}$, et $k=0,1, \ldots, n$, soit $d_{k}=d_{k}(X \mid \Delta):=\operatorname{dim}\left(S^{k}(X \mid \Delta)\right)$, et pour $k=1, \ldots, n$, soit $: d^{\prime}=d_{k}^{\prime}:=$ $\operatorname{dim}\left(R\left(S^{k}(X \mid \Delta)\right)\right)$.

Les $(2 n+1)$ entiers : $d_{0}:=\operatorname{dim}(X) \geq d_{1}^{\prime} \geq d_{1} \geq d_{2}^{\prime} \geq d_{2} \geq \cdots \geq d_{n}=$ $\operatorname{dim}\left((X \mid \Delta)\right.$ forment une suite décroissante $t(X \mid \Delta)=\left(d_{0}, d_{1}^{\prime}, \ldots, d_{n}\right)$ appelée le type de $(X \mid \Delta)$.

Soit $\nu=\nu(X \mid \Delta)$ le plus petit entier $k \leq n$ tel que $d_{k}=d_{k+1}=d_{n}$ ou $d_{k-1}^{\prime}=d_{k}^{\prime}=d_{n}$. Cet entier $\nu$ est la longueur de $(X \mid \Delta)$.

Le type de $(X \mid \Delta)$ est soumis aux conditions : $d_{k}>d_{k+1}^{\prime}$ si $k<\nu(X \mid \Delta)$ (puisque la composée de deux fibrations dont les fibres orbifoldes générales ont $\kappa_{+}=-\infty$ a aussi cette propriété).

Nous allons voir que ces conditions sont les seules, en général. Il nous suffira pour cela, par récurrence sur $\nu$, d'établir le lemme 10.7 suivant :

Lemme 10.7 Soit $X=(X \mid 0)$ une variété projective lisse de dimension $m$, de type $t=\left(m=\delta_{0}, \delta_{1}^{\prime}, \delta_{1}, \ldots, \delta_{\nu-1}=\cdots=\delta_{m}\right)$ et de longueur $(\nu-1)$. Alors, pour tout entier $r>0$ :

1. Si $\kappa(X) \geq 0,\left(\left(X \times \mathbb{P}^{r}\right) \mid 0\right)$ est de longueur $\nu$ et de type $\left(d_{0}=m+r, d_{1}^{\prime}=\right.$ $m=\delta_{0}=\delta_{1}, d^{\prime} j=\delta_{j-1}, d_{j}=\delta_{j-1}$ pour $\left.j=1, \ldots, m\right)$. 
2. Il existe une variété projective lisse $Y$ telle que $\kappa(Y)=\operatorname{dim}(X)=m$, $\operatorname{dim}(Y)=\operatorname{dim}(X)+r$, et une fibration $J: Y \rightarrow X$ qui est la fibration de Moishezon-Iitaka de $Y$. Le type de $Y$ est alors : $\left(m+r=d_{0}=d_{1}, d_{j}^{\prime}=\right.$ $\delta_{j-1}^{\prime}, d_{j}=\delta_{j-1}$ pour $\left.j=1, \ldots, m\right)$.

Démonstration : L'assertion 1 est évidente, puisque la projection de $Y:=\left(X \times \mathbb{P}^{r}\right)$ sur son premier facteur est le quotient rationnel de $Y$, et coincide donc avec $r$ pour l'orbifolde géométrique $(Y \mid 0)$.

Pour l'assertion 2, on choisit sur $X$ un fibré rès ample $H$, et pour $Y$ un membre générique du système linéaire $\left|d . H \times \mathcal{O}_{\mathbb{P}^{r+1}}(r+1)\right|$, sur $\left(X \times \mathbb{P}^{r+1}\right)$, pour $d>0$ entier assez grand. La projection de $Y$ sur $X$ induite par celle du produit sur son premier facteur n'a pas de fibre multiple en codimension 1 , et satisfait les conditions énoncées. (Nous ne le vérifions pas ici)

Exemple 10.8 Pour tout $n \geq 0$, on note $c(n)$ (tout comme en [Ca 04, 6.22]), le nombre de types en dimension $n$. Par étude directe on voit ([Ca 04, 6.22]) que $c(0)=1, c(1)=3, c(2)=8, c(3)=21$.

En dimension 1 , les trois types sont : $(1,0,0),(1,1,0)$ et $(1,1,1)$ correspondant respecivement $\grave{a} \kappa=-\infty, 0,1$ pour les courbes $(X \mid 0)$.

En dimension 2, les 8 types sont : $(2,0,0,0,0),(2,1,1,0,0),(2,1,1,1,1)$, $(2,2,0,0,0),(2,2,1,0,0),(2,2,1,1,0),(2,2,1,1,1),(2,2,2,2)$. Ils correspondent, pour les surfaces $(X \mid 0)$ aux invariants $(\kappa, \tilde{q})$ suivants respectivement, où q est le maximum des irrégularités des revêtements étales finis de $X:(-\infty, 0),(-\infty, 1),(-\infty,+\infty)$ (ie : $q \geq 2), \kappa=0,(\kappa=1,0),(\kappa=$ $1,1),(\kappa=1,+\infty), \kappa=2$.

On trouvera dans [Ca 04,3.38] une liste partielle des invariants birationnels des variétés $(X \mid 0)$ correspondants aux différents types non-spéciaux possibles en dimension 3.

Plus généralement, on va montrer $([\mathrm{Ca} 04,6.22])^{34}$ que : $c(n)=\frac{a^{n+1}-b^{n+1}}{\sqrt{5}}$, avec $: a=\frac{3+\sqrt{5}}{2}$ et $b=\frac{3-\sqrt{5}}{2}$, $\operatorname{car} c(n+1)=3 c(n)-c(n-1)$.

En effet : on a $c(n+1)=(n+2)+\sum_{0}^{n}\left(n+1-d_{1}\right) \cdot c\left(d_{1}\right)$. Le premier terme correspond aux types tels que $d_{1}^{\prime}=d_{1} \leq(n+1)$, le deuxième aux types tels que : $\left(d_{1}^{\prime}>d_{1}\right)$. On en déduit : $c\left(n+1-c(n)=1+\sum_{0}^{n} c\left(d_{1}\right)\right.$, puis l'égalité voulue en faisant la différence avec $c(n)-c(n-1)$.

\footnotetext{
${ }^{34}$ L'expression donnée de $c(n)$ est exacte, bien que la formule de récurrence y soit incorrecte!
} 
Remarque 10.9 Sous réserve de $C_{n, m}^{o r b}$, nous avons donc défini une série de nouveaux invariants biméromorphes des orbifoldes géométriques lisses $(X \mid \Delta)$. Ces invariants sont donc définis en particulier dans les cas extrêmes $\Delta=0$ (cas compact) et $\Delta=\operatorname{Supp}(\Delta)$ (cas ouvert, qui s'applique à toute compactification à croisements normaux de toute variété quasi-projective). Nous conjecturons que ces invariants entiers sont stables par déformation (par exemple lorsque $\Delta=0$ ).

Ces invariants généralisent et précisent la dimension canonique (dite "de Kodaira"). Ils fournissent, déjà lorsque $\Delta=0$, (voir, par exemple, le cas de la dimension 2 ci-dessus) une description beaucoup plus précise de la structure de $X$ que la classique dimension "de Kodaira".

\subsection{Analogie avec les algèbres de Lie.}

Dans le dictionnaire ci-dessous entre notions de géométrie complexe, et notions de la théorie des algèbres de Lie complexes, le "coeur" est l'analogue de la fibration de Levi-Malčev, tandis que la décomposition du coeur $c=$ $(M \circ r)^{n}$ est l'analogue de la suite des quotients par la série dérivée.

\begin{tabular}{|c||c|}
\hline Géométrie orbifolde & Algèbres de Lie \\
\hline \hline Fibration & Extension \\
\hline Fibre générale orbifolde & Noyau \\
\hline Base orbifolde stable & Quotient \\
\hline \hline Spéciale & Résoluble \\
\hline Type général & Semi-simple \\
\hline$\kappa=0$ ou $\kappa_{+}=-\infty$ & Abélienne \\
\hline \hline Coeur & Levi-Malčev \\
\hline
\end{tabular}

\subsection{Fonctorialité (conditionnelle)}

Des lemmes de fonctorialité 4.16 et 6.16 pour $r$ et $M$, on déduit immédiatemment celles de $s$ (réduction spéciale élémentaire) et du coeur :

Proposition 10.10 Soit $f:\left(X \mid \Delta_{X}\right) \rightarrow\left(Y \mid \Delta_{Y}\right)$ est un morphisme dans la catégorie méromorphe des orbifoldes géométriques lisses. On suppose que $X \in \mathcal{C}$. Soit $k \geq 0$ un entier. Notons (pour simplifier les notations) $\left[S_{X}^{k} \mid \Delta_{S_{X}^{k}}\right]$ et $\left[S_{Y}^{k} \mid \Delta_{S_{Y}^{k}}\right]$ respectivement les bases orbifoldes stables de $s_{\left(X \mid \Delta_{X}\right)}^{k}$ et de $s_{\left(Y \mid \Delta_{Y}\right)}^{k}$, supposées exister. 
Il existe alors un (unique) morphisme $s_{f}^{k}:\left[S_{X}^{k} \mid \Delta_{S_{X}^{k}}\right] \rightarrow\left[S_{Y}^{k} \mid \Delta_{S_{Y}^{k}}\right]$ tel que : $s_{f}^{k} \circ s_{\left(X \mid \Delta_{X}\right)}^{k}=s_{\left(Y \mid \Delta_{Y}\right)}^{k} \circ f$.

On $a: \nu\left(X \mid \Delta_{X}\right) \geq \nu\left(Y \mid \Delta_{Y}\right)$.

Si $\nu=\nu\left(X \mid \Delta_{X}\right)$, s ${ }_{f}^{\nu}$ induit donc un morphisme orbifolde (dans la catégorie biméromorphe) entre les bases orbifoldes des coeurs de $\left(X \mid \Delta_{X}\right)$ et de $\left(Y \mid \Delta_{Y}\right)$

Question 10.11 Si $u:(X \mid \Delta) \rightarrow\left(X^{\prime} \mid \Delta^{\prime}\right)$ est un morphisme orbifolde étale en codimension 1 , entre orbifoldes géométriques lisses de $\mathcal{C}$, les morphismes naturels induits par $u\left(r_{u}^{+}, M_{u}, s_{u}, s_{u}^{k}\right)$ sont-ils également (sur des représentants adéquats) étales en codimension 1 ? En particulier, la dimension des orbifoldes géométriques images doit être alors la même. Nous verrons (en 9.10) que cette dernière propriété est vérifiée pour le coeur, et l'est donc bien, en particulier, pour chaque étape de la décomposition.

\subsection{Relèvement de propriétés par dévissage.}

Soit $(X \mid \Delta)$ une orbifolde géométrique lisse spéciale. Admettons la conjecture $C_{n, m}^{o r b}$. Soit alors $s^{k}:(X \mid \Delta) \rightarrow S^{k}(X \mid \Delta)$ la suite de fibrations définies ci-dessus, pour $k \leq \nu(X \mid \Delta)$, avec $s^{k}=(M \circ r)^{k}$. Cette suite est appelée le dévissage canonique de $(X \mid \Delta)$.

Soit maintenant $(\mathrm{P})$ une propriété susceptible d'être ou non satisfaite par une orbifolde géométrique lisse $(X \mid \Delta)$, avec $X \in \mathcal{C}$. De manière équivalente, notons $(P)$ la classe des orbifoldes géométriques lisses $(X \mid \Delta)$, avec $X \in \mathcal{C}$ possédant la propriété $(\mathrm{P})$.

On a alors le lemme de "relèvement" évident suivant, qui permet de relever aux orbifoldes géométriques spéciales des propriétés satisfaites pour les classes $\kappa=0$ et $\kappa_{+}=-\infty$ :

Lemme 10.12 Admettons $C_{n, m}^{\text {orb }}$.

A. Supposons que la classe $(P)$ possède les propriétés de stabilité suivantes :

1. Invariance biméromorphe.

2. $(X \mid \Delta) \in(P)$ si $\kappa(X \mid \Delta)=0$.

3. $(X \mid \Delta) \in(P)$ si $\kappa_{+}(X \mid \Delta)=-\infty$.

4. Stabilité par extension : si $f:(X \mid \Delta) \rightarrow Y$ est une fibration méromorphe de base orbifolde stable $[Y \mid \Delta(f, \Delta)] \in(P)$, et si $(X \mid \Delta)_{y} \in(P)$, pour $y \in Y$ général, alors $(X \mid \Delta) \in(P)$. 
Alors : $(X \mid \Delta) \in(P)$ si $(X \mid \Delta)$ est lisse et spéciale.

B. Si, de plus, la classe $(P)$ est telle que:

5. $(X \mid \Delta) \notin(P)$ si $(X \mid \Delta)$ est lisse, de type général, avec : $\operatorname{dim}(X)>0$.

6. Si $f:(X \mid \Delta) \rightarrow Y$ est une fibration, avec $(X \mid \Delta) \in(P)$, alors $[Y \mid \Delta(f, \Delta)] \in(P)$.

Alors : $(P)$ est exactement la classe des orbifoldes géométriques spéciales.

(Pour démontrer le point B., utiliser le "coeur", en raisonnant par l'absurde).

Ce lemme devrait permettre de réduire la preuve d'un certain nombre de propriétés conjecturales (voir S.12) des orbifoldes géométriques lisses et spéciales aux cas "élémentaires" cruciaux $\kappa=0$ et $\kappa_{+}=-\infty$. La stabilité par extensions est elle-même conjecturale dans la plupart des situations. Sa signification est la suivante : si les obstructions à étendre $(P)$ s'annulent localement sur la base (orbifolde stable) de $f$, elles s'annulent globalement sur cette base. Des exemples de propriétés auxquelles appliquer ce principe de relèvement sont fournies dans le $\S 12$. 


\section{GROUPE FONDAMENTAL}

\subsection{Groupe fondamental d'une orbifolde géométrique lisse}

Les orbifoldes géométriques considérées dans cette section sont entières, lisses et connexes. Les morphismes orbifoldes le sont au sens divisible (voir définition 2.3).

On montre ici que le groupe fondamental orbifolde se comporte, sous l'hypothèse usuelle de lissité, comme dans le cas des variétés sans structure orbifolde (et même mieux, puisque les fibrations induisent toujours des suites exactes, voir 11.7. Ce fait est, avec 8.3, une seconde indication du fait que la catégorie orbifolde géométrique puisse être le cadre naturel de la géométrie kählérienne ou algébrique).

La définition suivante est classique :

Definition 11.1 Soit $(X \mid \Delta)$ une orbifolde géométrique lisse, avec $X$ connexe, et $\Delta:=\sum_{j \in J}\left(1-\frac{1}{m_{j}}\right) . D_{j}$. On note $X^{*}:=(X-\operatorname{supp}(\Delta))$ le complémentaire dans $X$ du support de $\Delta$, et $a \in X^{*}$. On note $\pi_{1}(X \mid \Delta, a)$ le quotient de $\pi_{1}\left(X^{*}, a\right)$ par le sous-groupe normal engendré par les lacets $g_{j}^{m_{j}}$, désignant par $g_{j}, \forall j \in j$ le lacet basé en a, tournant une fois dans le sens direct autour du diviseur $D_{j}$ (lacet dit "élémentaire" pour $D_{j}$ ). (Dans les problèmes considérés ici, on omettra la mention des points-base, qui n'y jouent aucun rôle).

On notera enfin $X^{0}$ tout ouvert de Zariski de $X$, complémentaire d'un sous-ensemble analytique fermé $A$ de codimension 2 au moins, $A$ contenant le lieu singulier Sing $(\lceil\Delta\rceil)$. On note $\Delta^{0}:=\Delta \cap X^{0}$.

Remarque 11.2 Si $X$ est compacte, $\pi_{1}(X \mid \Delta)$ est donc de présentation finie, puisque quotient de $\pi_{1}\left(X^{*}\right)$, qui est de type fini, par le sous-groupe normal engendré par un nombre fini d'éléments.

Proposition 11.3 Soit $f:\left(X \mid \Delta_{X}\right) \rightarrow\left(Y \mid \Delta_{Y}\right)$ un morphisme orbifolde divisible $^{35}$ entre orbifoldes géométriques lisses, avec $X$ connexe.

1. Il induit un morphisme fonctoriel naturel de groupes $f_{*}: \pi_{1}\left(X \mid \Delta_{X}\right) \rightarrow$ $\left(Y \mid \Delta_{Y}\right)$.

2. Si $f$ est une fibration, $f_{*}$ est surjectif.

\footnotetext{
${ }^{35}$ Voir définition en 2.3 .
} 
3. L'injection $j^{0}:\left(X^{0} \mid \Delta^{0}\right) \rightarrow(X \mid \Delta)$ induit un isomorphisme de groupes : $j_{*}^{0}: \pi_{1}\left(X^{0} \mid \Delta^{0}\right) \rightarrow \pi_{1}(X \mid \Delta)$

4. Si $f$ est étale en codimension $1, f_{*}$ est injectif. L'indice de $\pi_{1}\left(X \mid \Delta_{X}\right)$ dans $\pi_{1}\left(Y \mid \Delta_{Y}\right)$ est égal au degré géométrique de $f$.

5. Si $f$ est propre et surjective, son image est d'indice fini (au plus égal au nombre de composantes connexes d'une fibre générique) dans $\pi_{1}\left(Y \mid \Delta_{Y}\right)$.

Démonstration : 1 . L'image par $f$ du support $D_{X}$ de $\Delta_{X}$ est contenue dans le support $D_{Y}$ de $\Delta_{Y}$. Si $D$ est une composante de $\Delta_{X}$ de multiplicité $m$, et si $E$ est une composante quelconque de $\Delta_{Y}$ contenant $f(D)$, de multiplicité $m^{\prime}$, on a donc, par hypothèse : $t . m=k . m^{\prime}$, pour un entier $k>0$. Soit donc $g_{D}$ un lacet de $X-D_{X}$ élémentaire pour $D$. Soit $g_{E}$ un lacet élémentaire relatif à $E$ dans $\left(Y-D_{Y}\right)$. Puisque $f^{*}(E)=t . D+\ldots, f_{*}\left(g_{D}\right)=\left(g_{E}\right)^{t}$ (à conjugaison près dans $\left.\pi_{1}\left(Y-D_{Y}\right)\right)$. Donc $f_{*}\left(g_{D}^{m}\right)=\left(g_{E}\right)^{t \cdot m}=\left(g_{E}\right)^{m^{\prime} \cdot k}=$ $\left(\left(g_{E}\right)^{m^{\prime}}\right)^{k}$, est donc dans le noyau du quotient $\pi_{1}\left(Y-D_{Y}\right) \rightarrow \pi_{1}\left(Y \mid \Delta_{Y}\right)$, puisque ceci est vrai pour toute composante $E$ de $D_{Y}$. La fonctorialité de $f_{*}$ résulte immédiatemment de sa définition.

2. Soit $X^{* *}:=\left(X-f^{-1}\left(D_{Y}\right)\right) \cap X^{*} \subset X^{*} \subset X$. Si $f$ est une fibration, le morphisme de groupes $\pi_{1}\left(X^{* *}\right) \rightarrow \pi_{1}\left(Y^{*}\right)$ est surjectif. En effet $: X$, et donc $X^{* *}$ est lisse et connexe, les fibres génériques de $f_{\mid X^{* *}}: X^{* *} \rightarrow Y^{*}$ sont connexes, et $f_{\mid X^{* *}}: X^{* *} \rightarrow Y^{*}$ est surjective, par hypothèse. Donc $f_{*}$ est également surjectif, puisque $f_{*}$ est déduit du morphisme de groupes précédent par passage aux quotients. Plus précisément, le diagramme commutatif cidessous établit la surjectivité de $f_{*}$.

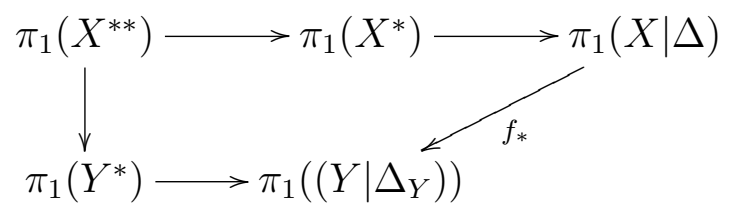

3. Les groupes $\pi_{1}\left(X^{*}\right)$ et $\pi_{1}\left(X^{0}\right)^{*}$ sont naturellement isomorphes par $j^{0}$, et les noyaux des morphismes définissant $\pi_{1}\left(X^{0} \mid \Delta^{0}\right)$ et $\pi_{1}(X \mid \Delta)$ sont engendrés par les (classes des) lacets élémentaires correspondants.

4. On est donc réduit, par 3 , au cas où $f$ est étale au sens orbifolde.

On peut donc supposer être dans la situation de 2.22. Si $D$ est une composante de $D_{X}$ de multiplicité $m$, alors $f(D)=E$ est une composante du support de $D_{Y}$ qui est de multiplicité $m^{\prime}$, avec $r . m=m^{\prime}$, si $r$ est l'indice de ramification de $f$ le long de $D$, tel donc que $f^{*}(E)=r . D+\ldots$ Donc $f_{*}\left(g_{D}\right)^{m}=\left(\left(g_{E}\right)^{r}\right)^{m^{\prime}}$, avec les notations utilisées dans la preuve de 1 . Puisque 
le morphisme naturel $\pi_{1}\left(X^{*}\right) \rightarrow \pi_{1}\left(Y^{*}\right)$ induit par la restriction de $f$ est injectif, et que les sous-groupes normaux de $\pi 1\left(Y^{*}\right)$ engendrés par les lacets élémentaires autour des composantes de $D_{X}$ et de $D_{Y}$ coincident, $\pi_{1}\left(X \mid \Delta_{X}\right)$ est bien un sous-groupe de $\pi_{1}\left(Y \mid \Delta_{Y}\right)$ dont l'indice coincide avec celui de $\pi_{1}\left(X^{*}\right)$ dans $\pi_{1}\left(Y^{*}\right)$, égal au degré (fini ou pas) de $f$.

5. Le diagramme utilisé dans la démonstration de l'assertion 2 ci-dessus montre que l'on peut remplacer $Y$ par l'un quelconque de ses ouverts non vides de Zariski $Y^{\prime}$, et $X$ par $X^{\prime}:=f^{-1}\left(Y^{\prime}\right)$. Soit $f=h \circ s, s: X \rightarrow Z$ une fibration, et $h: Z \rightarrow Y$ finie, la factorisation de Stein de $f$. Quitte à remplacer $Y$ par $Y^{\prime}$ adéquat, on supposera que $\left(Z \mid \Delta\left(s, \Delta_{X}\right)\right)$ est lisse, que $s_{\Delta}:\left(X \mid \Delta_{X}\right) \rightarrow\left(Z \mid \Delta\left(s, \Delta_{X}\right)\right)$ est un morphisme orbifolde (nécessairement divisible), et que $h: Z \rightarrow Y$ est étale et induit un morphisme orbifolde divisible $h_{\Delta}:\left(Z \mid \Delta_{Z}\right):=\left(Z \mid \Delta\left(s, \Delta_{X}\right)\right) \rightarrow\left(Y \mid \Delta_{Y}\right)$. La seule assertion non immédiate est la divisibilité de $s_{\Delta}$ et $h_{\Delta}$. Puisque $f$ est divisible, $\Delta_{Y}$ divise $\Delta\left(f, \Delta_{X}\right)$. Par finitude de $h, \Delta\left(f, \Delta_{X}\right)=\Delta\left(h, \Delta\left(s, \Delta_{X}\right)\right):=\Delta\left(h, \Delta_{Z}\right)$. Ce sont les assertions de divisibilité annoncées. On est donc réduit au cas où $f=s$ est étale finie. L'assertion de divisibilité signifie alors que $h^{*}\left(\Delta_{Y}\right)$ divise $\Delta_{Z}$. Donc $\pi_{1}\left(Z / h^{*}\left(\Delta_{Y}\right)\right)$ est un quotient de $\pi_{1}\left(Z \mid \Delta_{Z}\right)$. Puisque $\pi_{1}\left(Y \mid \Delta_{Y}\right)$ est un quotient de $\pi_{1}\left(Z / h^{*}\left(\Delta_{Y}\right)\right)$ de degré égal au nombre de composantes connexes de la fibre générique de $f$, le résultat est établi.

Remarque 11.4 1. La condition de divisibilité du morphisme orbifolde $f$ est en général nécessaire, pour l'existence de $f_{*}$ : considérer par exemple $X=\mathbb{P}^{1}$ avec les trois diviseurs orbifoldes $\Delta_{k}=\{0\}+\left(1-\frac{1}{a_{k}}\right) \cdot\{\infty\}$, pour $k=1,2,3$ et $a_{1}<a_{2}<a_{3}=+\infty$, $a_{1}$ et $a_{2}$ premiers entre eux. Ils induisent deux morphismes de groupes $: \mathbb{Z} \rightarrow \mathbb{Z}_{a_{1}}$ et $\mathbb{Z} \rightarrow \mathbb{Z}_{a_{2}}$, mais aucun morphisme $\mathbb{Z}_{a_{2}} \rightarrow \mathbb{Z}_{a_{1}}$ n'est compatible par composition avec les précédents.

2. Si $f:(X \mid \Delta) \rightarrow(Y \mid \Delta)$ est l'éclatement d'un point lisse du support de $\Delta_{Y}$, situé sur une composante $D$ de $D_{Y}$ affectée dans $\Delta_{Y}$ de la multiplicité $m>1$, et si le diviseur exceptionnel $D$ de $f$ est affecté dans $\Delta_{X}$ tel que $f_{*}\left(\Delta_{X}\right)=\Delta_{Y}$ d'une multiplicité $m^{\prime}$, première avec $m$, et assez grande pour que $f:(X \mid \Delta) \rightarrow(Y \mid \Delta)$ soit un morphisme orbifolde (non divisible), alors $\pi_{1}\left(X \mid \Delta_{X}\right) \cong \pi_{1}\left(Y \mid \Delta_{Y}^{\prime}\right)$, où $\Delta_{Y}^{\prime}$ est obtenu de $\Delta_{Y}$ par suppression de la composante $(1-1 / m)$.D. Ce dernier groupe est en général un quotient strict de $\pi_{1}\left(Y \mid \Delta_{Y}\right)$.

En particulier, le groupe fondamental n'est pas un invariant biméromorphe dans la catégorie des orbifoldes lisses (munie des morphismes non divisibles). 
3. Un cas particulier de $f:\left(X \mid \Delta_{X}\right) \rightarrow\left(Y \mid \Delta_{Y}\right)$ auquel l'assertion 2 peut être appliquée est celui dans lequel $X=Y$ et $\Delta_{Y}$ divise $\Delta_{X}$ (ie : $m_{\Delta_{Y}}(D)$ divise $\left.m_{\Delta_{X}}(D), \forall D \in W(X)=W(Y)\right): \pi_{1}\left(Y \mid \Delta_{Y}\right)$ est alors un quotient de $\pi_{1}\left(X \mid \Delta_{X}\right)$.

Proposition $11.5 f:\left(X \mid \Delta_{X}\right) \rightarrow\left(Y \mid \Delta_{Y}\right)$ un morphisme orbifolde divisible, avec $X$ connexe. On suppose que $f$ est biméromorphe au sens orbifolde (ie : biméromorphe de $X$ sur $Y$, et tel que $f_{*}\left(\Delta_{X}\right)=\Delta_{Y}$ ). Alors $f_{*}$ est un isomorphisme de groupes.

En particulier, deux orbifoldes géométriques biméromorphes au sens divisible $^{36}$ ont des groupes fondamentaux isomorphes.

Démonstration : Par l'observation faite ci-dessus, on ne change pas $\pi_{1}\left(Y \mid \Delta_{Y}\right)$ en restreignant $Y$ au complémentaire de $A \subset Y$, Zariski fermé de codimension au moins 2. On choisit $A$ contenant le lieu d'indétermination de $f^{-1}$. Alors $f^{\prime}: X^{*} \rightarrow Y^{*}$ est un isomorphisme. Et il existe donc un morphisme quotient $h: \pi_{1}\left(Y^{*}\right) \rightarrow \pi_{1}\left(X \mid \Delta_{X}\right)$ tel que $f_{*} \circ h=\delta_{Y}: \pi_{1}\left(Y^{*}\right) \rightarrow$ $\pi_{1}\left(Y \mid \Delta_{Y}\right)$ est le quotient naturel. Puisque le noyau de $\delta$ (engendré par les lacets $\Delta_{Y}$-élémentaires) est contenu dans celui de $h$ (engendré par les lacets $\Delta_{X}$-élémentaires), $f_{*}$ est un isomorphisme

Definition 11.6 Soit $\Delta^{\prime}$ et $\Delta$ deux diviseurs orbifoldes sur $X$, connexe. Alors $i d_{X}:\left(X \mid \Delta_{X}\right) \rightarrow\left(X \mid \Delta^{\prime}\right)$ est un morphisme orbifolde divisible si et seulement si $\Delta^{\prime}$ divise $\Delta$ (ie : que pour chaque $D \in W(X), m_{\Delta^{\prime}}(D)$ divise $\left.m_{\Delta}(D)\right)$. Ce morphisme induit un morphisme de groupes $\left(i d_{X}\right)_{*}$ : $\pi_{1}\left(X \mid \Delta_{X}\right) \rightarrow \pi_{1}\left(X \mid \Delta^{\prime}\right)$ surjectif.

On dit que $(X \mid \Delta)$ est régulière si le seul diviseur orbifolde $\Delta^{\prime}$ divisant $\Delta$, tel que le $\left(i d_{X}\right)_{*}$ soit un isomorphisme est $\Delta^{\prime}=\Delta$ (autrement dit : si on ne peut pas "réduire" strictement $\Delta$ sans réduire strictement son $\pi_{1}$ ).

\subsection{Suite exacte associée à une fibration orbifolde nette}

Proposition $11.7 f:\left(X \mid \Delta_{X}\right) \rightarrow\left(Y \mid \Delta_{Y}\right)$ un morphisme orbifolde divisible, avec $X$ connexe. Soit $\left(X_{y} \mid \Delta_{y}\right)$ une fibre orbifolde lisse générique de $f$,

\footnotetext{
${ }^{36}$ ie : s'il existe une chaine d'équivalences biméromorphes orbifoldes biméromorphes divisibles les reliant.
} 
notant $\Delta:=\Delta_{X}$, et $j:\left(X_{y} \mid \Delta_{y}\right) \rightarrow(X \mid \Delta)$ l'inclusion naturelle. Elle induit une morphisme de groupes naturel $j_{*}: \pi_{1}\left(X_{y} \mid \Delta_{y}\right) \rightarrow \pi_{1}(X \mid \Delta)$.

Si $f$ est nette au sens divisible ${ }^{37}$, la suite de groupes suivante induite par $j$ et $f$ est exacte :

$$
\pi_{1}\left(X_{y} \mid \Delta_{y}\right) \stackrel{j_{*}}{\longrightarrow} \pi_{1}(X \mid \Delta) \stackrel{f_{*}}{\longrightarrow} \pi_{1}\left(Y \mid \Delta_{Y}\right) \longrightarrow\{1\}
$$

Démonstration : Seule l'exactitude en $\pi_{1}(X \mid \Delta)$ reste à établir. Puisque $f_{*} \circ j_{*}=(f \circ j)_{*}$, il faut montrer que le noyau de $f_{*}$ est contenu dans l'image de $j_{*}$. Rappelons (voir 3.8) que $f$ "nette" (au sens divisible) signifie qu'il existe un diagramme commutatif :

dans lequel :

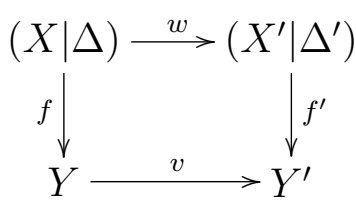

1. $w$ est un morphisme orbifolde divisible, $v$ et $w$ étant biméromorphes, $Y, Y^{\prime}$ lisses, et $w_{*}(\Delta)=\Delta^{\prime}$.

2. Tout diviseur $g$-exceptionnel de $X$ est $w$-exceptionnel.

Si $A \subset Y$ est analytique fermé de codimension 2 ou plus, $f^{-1}(A)=$ $B \cup E \subset X$ est analytique fermé réunion de $B$, et $E^{\prime}$, analytique fermés dans $X, B$ de codimension 2 ou plus, et $E$ un diviseur $f$-exceptionnel, donc $w$-exceptionnel, puisque $f$ est nette relativement à $f^{\prime}$. Il en résulte que $\pi_{1}\left(X " \mid \Delta^{\prime \prime}\right)=\pi_{1}(X \mid \Delta)$, si $X "=f^{-1}(Y-A)$, et $\Delta^{\prime \prime}:=\Delta \cap X^{\prime}$.

Remplaçant $Y$ par un ouvert adéquat $Y$ " et $X$ par $X^{\prime \prime}:=f^{-1}\left(Y^{\prime \prime}\right)$, on supposera donc désormais que $f$, ainsi que sa restriction au support de $\Delta_{X}$ sont à fibres équidimensionnelles, le support de $\Delta_{Y}$ étant lisse.

On va maintenant montrer que l'on peut supposer que, de plus, $\Delta=\Delta^{\text {vert }}$, partie $f$-verticale de $\Delta$, ce dernier diviseur orbifolde étant simplement la réunion des composantes du support de $\Delta$ qui ne sont pas envoyées surjectivement sur $Y$ par $f$, les multiplicités des composantes ( $f$-verticales) restantes étant conservées.

Alors : $\Delta_{Y}:=\Delta(f, \Delta)=\Delta\left(f, \Delta^{v e r t}\right)$. Cette dernière égalité résulte immédiatemment des définitions.

On a, de plus, une surjection naturelle $\pi_{1}(X \mid \Delta) \rightarrow \pi_{1}\left(X \mid \Delta^{\text {vert }}\right)$, compatible avec les restrictions à $X_{y}$.

\footnotetext{
37ie : satisfait la condition de 3.8, les morphismes orbifoldes l'étant alors tous au sens divisible.
} 
La propriété cruciale pour cette seconde réduction est le fait que le noyau du morphisme naturel $\pi_{1}(X \mid \Delta) \rightarrow \pi_{1}\left(X \mid \Delta^{\text {vert }}\right)$ est engendré par les lacets élémentaires autour des composantes $f$-horizontales de $\Delta$, et est donc contenu dans $\pi_{1}\left(X_{y} \mid \Delta_{X_{y}}\right)=\pi_{1}\left(X_{y}\right)$, puisque $\Delta_{\mid X_{y}}^{\text {vert }}=0$.

On supposera donc que $f$ et sa restriction à $\Delta=\Delta^{\text {vert }}$ sont équidimensionnels, et que $\Delta_{Y}=\Delta(f, \Delta)$ est à support lisse.

Soit donc $g \in \pi_{1}\left(X^{*}\right)$ tel que son image dans $\pi_{1}(X \mid \Delta)$ soit dans le noyau de $f_{*}$ ci-dessus. On peut donc écrire : $f(g)=\Pi_{r} h_{r}^{p_{r}} \cdot \mu_{r}$, où les $h_{r}^{\mu_{r}}$ sont des lacets élémentaires autour des composantes du support de $\Delta_{Y}$. Puisque $\Delta_{Y}$ est la base orbifolde de $(f \mid \Delta)$, pour chaque $r$, on peut écrire, par le théorème de Bezout : $h_{r}^{\mu_{r}}=\Pi_{s} f\left(g_{r, s}^{t_{r, s} \cdot m_{r, s} \cdot q_{r, s}}\right)$, puisque $\mu_{r}=\operatorname{pgcd}_{s}\left(t_{r, s} \cdot m_{r, s}\right)$, par la définition de la base orbifolde (divisible). Les $g_{r, s}$ sont des lacets élémentaires autour de composantes de $\Delta^{\text {vert }}$ dont l'image par $f$ est la composante d'indice $r$, de multiplicité $\mu_{r}$, du support de $\Delta_{Y}$.

Donc $: g=\prod_{r, s} f\left(g_{r, s}^{p_{r} \cdot t_{r, s} \cdot m_{r, s} \cdot q_{r, s}}\right) \cdot g^{\prime}$, où $f\left(g^{\prime}\right)=1$ dans $\pi_{1}\left(Y^{*}\right)$. Mais la suite exacte d'homotopie :

$$
\pi_{1}\left(X_{y}\right) \stackrel{j_{*}}{\longrightarrow} \pi_{1}\left(X^{*}\right) \stackrel{f_{*}}{\longrightarrow} \pi_{1}\left(Y^{*}\right) \longrightarrow\{1\}
$$

montre que $g^{\prime} \in \pi_{1}\left(X_{y}\right)=\pi_{1}\left(X_{y} \mid \Delta_{X_{y}}\right)$. Ce qui achève a démonstration.

Passant à un modèle holomorphe net, on en déduit la version biméromorphe suivante :

Corollaire 11.8 Soit $(X \mid \Delta)$ lisse, avec $X$ connexe, et $f: X \rightarrow Y$ méromorphe dominante connexe et propre, de fibre orbifolde générique $(X \mid \Delta)_{y}$ (sur un modèle biméromorphe rendant $f$ holomorphe), et de base orbifolde stable $\left[Y \mid \Delta_{Y}\right]$. La suite de groupes suivante est alors exacte:

$$
\pi_{1}\left(X_{y} \mid \Delta_{y}\right) \stackrel{j_{*}}{\longrightarrow} \pi_{1}(X \mid \Delta) \stackrel{f_{*}}{\longrightarrow} \pi_{1}\left(\left[Y \mid \Delta_{Y}\right]\right) \longrightarrow\{1\}
$$

Explicitons le lien entre le groupe fondamental de $X \in \mathcal{C}$, lisse et connexe, et celui de la base orbifolde (stable) de sa $\Gamma$-réduction.

Corollaire 11.9 Soit $X \in \mathcal{C}$, lisse et connexe. Soit $\gamma_{X}: X \rightarrow Y:=\Gamma(X)$ sa $\Gamma$-réduction (au sens de [ca94], ou sa réduction de Shafarevich), et $X_{g}$ sa fibre générique (lisse). Soit $\left(\Gamma(X) \mid \Delta\left(\gamma_{X}\right)\right)=\left[Y \mid \Delta_{Y}\right]$ sa base orbifolde (stable). Alors on a une suite exacte de groupes :

$$
\pi_{1}\left(X_{g}\right) \stackrel{j_{*}}{\longrightarrow} \pi_{1}(X) \stackrel{\gamma_{*}}{\longrightarrow} \pi_{1}\left(\left[Y \mid \Delta_{Y}\right]\right) \longrightarrow\{1\}
$$

Remarque 11.10 Cette suite exacte montre que l'étude de $\pi_{1}(X)$ nécessite celle de l'orbifolde géométrique $\left(Y \mid \Delta_{Y}\right)$. 


\subsection{Revêtement universel d'une orbifolde géométrique lisse}

Soit $(X \mid \Delta)$ une orbifolde géométrique lisse avec $X$ connexe. On note $\Delta=\sum_{j \in J}\left(1-1 / m_{j}\right) \cdot D_{j}$. On note aussi $X^{*}:=(X-\lceil\Delta\rceil)$, et $X^{0}:=X-A$, avec $A \supset \operatorname{Sing}(\lceil\Delta\rceil)$ le lieu singulier du support de $\Delta$, si $A$ est de codimension 2 au moins dans $X$.

Nous adoptons ici la terminologie de [N87], et en rappellons certains résultats.

Definition 11.11 Un revêtement ramifié en au plus $\Delta$ de $X$ est une application holomorphe $g: \bar{X} \rightarrow X$ telle que :

1. $\bar{X}$ est normal et connexe, et les fibres de $g$ sont discrètes.

2. La restriction $g^{*}: \bar{X}^{*}:=g^{-1}\left(X^{*}\right) \rightarrow X^{*}$ de $g$ à $\bar{X}^{*}$ est un revêtement non ramifié (de groupe $G \subset \pi_{1}\left(X^{*}\right)$ ).

3. Si $\bar{D}_{j}^{0}:=g^{-1}\left(D_{j}\right) \subset \bar{X}^{0}:=g^{-1}\left(X^{0}\right)$, alors $g$ ramifie à l'ordre $n_{j}$, diviseur de $m_{j}$ le long de $\bar{D}_{j}^{0}$.

4. Tout $a \in X$ a un voisinage ouvert connexe $W_{a}$ tel que, pour toute composante connexe $U_{a}$ de $g^{-1}(W), U_{a} \cap g^{-1}(a)$ soit réduit à un point $\bar{a}$, et $g_{\mid U_{a}}: U_{a} \rightarrow W$ est propre (et finie).

On dira que $\bar{X}$ est le complété de $\bar{X}^{*}$ au-dessus du support de $\Delta$.

On dit que $g$ ramifie en $\Delta$ si $n_{j}=m_{j}, \forall j \in J$.

La notion d'isomorphisme de revêtements ramifiés est la notion usuelle. Les revêtements ramifiés sont considérés à isomorphisme près dans la suite.

Théorème 11.12 [N87, 1.3.8] Dans la situation précédente ${ }^{38}$, il existe une bijection naturelle entre les sous-groupes de $\pi_{1}\left(X^{*}\right)$ contenant le sous-groupe normal $K$ engendré par les $g_{j}^{m_{j}}, j \in J$, et les revêtements ramifiés en au plus $\Delta$ de $X$ (à isomorphisme de revêtement près).

Cette bijection associe au revêtement $g: \bar{X} \rightarrow X$ le sous-groupe de $\pi_{1}\left(X^{*}\right)$ consitué des automorphismes du revêtement $g^{*}: \bar{X}^{*} \rightarrow X^{*}$ obtenu par restriction de $g$ au-dessus de $X^{*}$. Réciproquement, à un sous-groupe, le théorème associe la complétion au-dessus du support de $\Delta$ du revêtement de $X^{*}$ défini par ce sous-groupe.

\footnotetext{
${ }^{38}$ Le résultat de [N87] couvre, plus généralement, les diviseurs orbifoldes à groupes fondamentaux locaux finis.
} 
Corollaire $11.13 \mathrm{Il}$ existe un unique revêtement $u_{X \mid \Delta}: \overline{(X \mid \Delta)}:=\bar{X} \rightarrow X$ ramifié en au plus $\Delta$, avec $\bar{X}^{0}$ (et donc a fortiori $\bar{X}$ ) simplement connexe. On l'appelle le revêtement universel de $(X \mid \Delta)$. Alors :

1. Pour tout $j \in J$, soit $n_{j}$ l'ordre auquel ramifie $u_{X \mid \Delta}$ le long de $\bar{D}_{j}^{0}$ : c'est un diviseur de $m_{j}$. Soit $\bar{\Delta}:=\sum_{j \in J}\left(1-1 / n_{j}\right) . D_{j}$ : ce diviseur orbifolde divise $\Delta$, et on l'appelle la régularisation de $\Delta$.

2. L'identité de $X$ induit un morphisme orbifolde (divisible) $i d_{X}$ : $(X \mid \Delta) \rightarrow(X / \bar{\Delta})$ qui est un isomorphisme au niveau des $\pi_{1}$. De plus :

3. $u_{X \mid \Delta}: \bar{X} \rightarrow X$ est aussi le revêtement universel de $(X / \bar{\Delta})$, et $u_{X \mid \Delta}$ ramifie en $\bar{\Delta}$. On $a: \overline{(\bar{\Delta})}=\bar{\Delta}$. En particulier $: \bar{\Delta}=\Delta$ si et seulement si $(X \mid \Delta)$ est régulière.

4. Le sous groupe normal de $\pi_{1}\left(X^{*}\right)$ engendré par les $g_{j}^{m_{j}}, j \in J$ est aussi le groupe normal engendré par les $g_{j}^{n_{j}}$ (notations de 11.11).

5. Si $f:\left(X^{\prime} \Delta^{\prime}\right) \rightarrow(X \mid \Delta)$ est un morphisme orbifolde divisible biméromorphe, alors le revêtement universel de $\left(X^{\prime} \mid \Delta^{\prime}\right)$ est déduit de celui de $(X \mid \Delta)$ par le changement de base $f: X^{\prime} \rightarrow X$ et normalisation.

Démonstration : Le revêtement universel est donc obtenu en considérant le sous groupe $K$. L'assertion 1 est évidente, d'après les définitions. L'isomorphisme au niveau des $\pi_{1}$ résulte de ce que les groupes $K$ coincident pour $\Delta$ et sa régularisation. L'assertion 3 est évidente. L'assertion 4 résulte de la définition du groupe $K$ associé à la régularisation de $\Delta$, et de ce qu'il coincide avec celui associé à $\Delta$. La propriété 5 . résulte de ce que les fibres des deux espaces coincident au-dessus de $X^{0}$, de ce que l'on a un morphisme naturel du changement de base normalisé $\overline{X^{\prime} \times_{X} \bar{X}} \rightarrow \bar{X}^{\prime}$ vers $\bar{X}^{\prime}$ au-dessus de $X$, et de ce que les fibres des deux espaces au-dessus de $X$ sont discrètes.

Remarque 11.14 Dans la situation de 11.13, on peut montrer l'assertion suivante (non utilisée dans la suite) :

Soit $f:\left(Y \mid \Delta_{Y}\right) \rightarrow(X \mid \Delta)$ une application biméromorphe propre, avec des orbifoldes géométriques lisses, $Y$ et $X$ étant connexes. On note $X^{0}=X-A$, $A \subset X$ étant de codimension 2 au moins, Zariski fermé, et contenant le lieu d'indétermination de $f^{-1}$ et $\operatorname{Sing}(\lceil\Delta\rceil)$.

On suppose que $f_{*}\left(\Delta_{Y}\right)=\Delta$, que $(X \mid \Delta)$ est régulière, et que l'isomorphisme $f^{0}: Y_{0}:=f^{-1}\left(X^{0}\right) \rightarrow X^{0}$ induit un isomorphisme entre ses quotients $\pi_{1}(X \mid \Delta)$ et $\pi_{1}\left(Y \mid \Delta_{Y}\right)$.

Alors $f$ est un morphisme orbifolde divisible. 
Concernant le revêtement universel de $X \in \mathcal{C}$, de 11.9, on déduit :

Proposition 11.15 Soit $X \in \mathcal{C}$, lisse et connexe. Soit $\gamma_{X}: X \rightarrow Y:=$ $\Gamma(X)$ sa $\Gamma$-réduction (au sens de [Ca94]), ou réduction de Shafarevich. Soit $\left(\Gamma(X) \mid \Delta\left(\gamma_{X}\right)\right)=\left[Y \mid \Delta_{Y}\right]$ sa base orbifolde (stable), et $u_{Y}: \bar{Y} \rightarrow Y$ son revêtement universel orbifolde. On suppose que l'on a modifié $X$ et $Y$ de telle sorte que $\gamma$ soit holomorphe et nette. Donc $\left(Y \mid \Delta\left(\gamma_{X}\right)\right)=\left[Y \mid \Delta_{Y}\right]$.

D'après 11.9 , on a une suite exacte:

$$
\pi_{1}\left(X_{g}\right) \stackrel{j_{*}}{\longrightarrow} \pi_{1}(X) \stackrel{\left(\gamma_{X}\right)_{*}}{\longrightarrow} \pi_{1}\left(Y \mid \Delta_{Y}\right) \longrightarrow\{1\}
$$

Soit $u: \bar{X} \rightarrow X$ le revêtement Galoisien de groupe $\pi_{1}\left(Y \mid \Delta_{Y}\right)$, quotient de $\pi_{1}(X)$ par le groupe normal fini $\pi_{1}\left(X_{g}\right)$.

Soit $X^{\prime}=X \times_{Y} \bar{Y}$ le produit fibré normalisé. Alors : le morphisme naturel $X^{\prime} \rightarrow X$ se relève en un morphisme propre et biméromorphe $\beta: X^{\prime} \rightarrow \bar{X}$.

Démonstration : Soit $Y_{0} \subset Y$ l'ouvert au-dessus duquel $\gamma_{X}$ est équidimensionnel, et $\left.\Delta\left(\gamma_{X}\right)\right)$ lisse. Soit $\bar{Y}_{0}$ son image réciproque dans $\bar{Y}$. Soit $X_{0}:=\gamma_{X}^{-1}\left(Y_{0}\right)$. Puisque $\gamma_{X}$ est supposée nette, $\pi_{1}\left(X_{0}\right) \cong \pi_{1}(X)$. Il nous suffit de voir que, au-dessus de $Y_{0}$, l'application naturelle $X^{\prime} \rightarrow X$ est étale. Soit $\left(Y \mid \Delta_{Y}^{\prime}\right)$ l'orbifolde géométrique régularisée de $\left(Y \mid \Delta_{Y}\right)$. Donc $\Delta_{Y}^{\prime}$ divise $\Delta_{Y}$, et a le même $\pi_{1}$ et le même revêtement universel. Puisque $\Delta_{Y}^{\prime}$ divise $\Delta_{Y}$, un calcul local direct montre que la normalisation de $X \times_{Y} \bar{Y}$ est étale au-dessus de $X_{0}$. (En effet, chaque composante de chaque fibre de $\gamma_{X}$ au-dessus de $Y_{0}$ a une multiplicité multiple de la multiplicité de $\Delta_{Y}^{\prime}$ au point image par $\gamma_{X}$ de cette fibre).

\subsection{Finitude résiduelle et critère d'abélianité.}

De 11.13 on déduit :

Corollaire 11.16 1. Il existe un revêtement ramifié (Galoisien) fini $g: \bar{X} \rightarrow$ $X$ qui ramifie en $\Delta$ (exactement) si et seulement s'il existe un sous-groupe (normal) $G^{\prime}$ d'indice fini $G$ de $\pi_{1}(X \mid \Delta)$ ne contenant, pour chaque $j \in J$, aucun des éléments $g_{j}^{k}$, pour $0<k<m_{j}$. Cette condition est satisfaite, en particulier, si $\pi_{1}(X \mid \Delta)$ est résiduellement fini.

2. S'il existe un revêtement ramifié (Galoisien) fini $g: \bar{X} \rightarrow X$ qui ramifie en $\Delta$ (exactement), alors la complétion au-dessus de $A \subset X$ du revêtement universel (au sens usuel) de $\bar{X}^{0}$ est le revêtement universel de $(X \mid \Delta)$. 
Démonstration : 1 . Soit $G^{\prime}<G$ un tel groupe, et $G^{*}<\pi_{1}\left(X^{*}\right)$ son image réciproque. Elle définit un revêtement étale fini $X^{* *}$ de $X^{*}$. Le groupe $G^{*}$ contient $K$, et définit donc un revêtement ramifiant en au plus $\Delta$. Il ramifie en $\Delta$ exactement, puisque, par hypothèse, les lacets $g^{n_{j}}$ ne sont pas dans $G^{\prime}$ si $n_{j}$ est un diviseur strict de $m_{j}$.

Si $\pi_{1}(X \mid \Delta)$ est résiduellement fini, un tel sous-groupe existe (par définition).

2. Dans ce cas, le revêtement universel de $\bar{X}_{0}$ satisfait les conditions de 11.13 caractérisant le revêtement universel de l'orbifolde géométrique $(X \mid \Delta)$.

Remarque 11.17 1. Dans la situation de 11.16, si $\sigma: \tilde{X} \rightarrow \bar{X}$ est une résolution des singularités (quotient) de $\bar{X}$, comme ci-dessus, alors l'inclusion de $\bar{X}^{0} \subset \tilde{X}$ induit un morphisme $: j_{*}: \pi_{1}\left(\bar{X}^{0}\right) \rightarrow \pi_{1}(\tilde{X})$ surjectif, mais de noyau infini, en général, comme le montre l'exemple (classique) suivant : $E$ une courbe elliptique, (-1) l'involution usuelle sur $E,(X \mid \Delta)=\left(\left(\mathbb{P}_{1} \times \mathbb{P}_{1}\right) \mid \Delta\right)$ le quotient de $E \times E$ par le sous-groupe à quatre éléments engendré par les deux involution agissant sur chacun des facteurs. On choisit pour $\bar{X}$ la surface de Kummer quotient de $E \times E$ par l'action diagonale des involutions. Néammoins, lorsque les groupes fondamentaux locaux des singularités de $\bar{X}$ sont triviaux, ce morphisme $j_{*}$ est bijectif. Nous exploiterons ce fait dans 11.18 ci-dessous.

2. En général, $\bar{X}$ est singulier. Par exemple, si $\Delta:=(1-1 / m) \cdot\left(D_{1}+D_{2}\right)$ est le diviseur orbifolde sur $\mathbb{P}^{2}$, avec $D_{1}, D_{2}$ deux droites projectives distinctes et $2 \leq m<+\infty$ entier, alors $\pi_{1}\left(\mathbb{P}^{2} \mid \Delta\right) \cong \mathbb{Z}_{m}$. De plus, $\bar{X}$, le revêtement universel orbifolde de $\left(\mathbb{P}^{2} \mid \Delta\right)$ est le cône sur la courbe rationnelle normale de degré $m$ dans $\mathbb{P}^{m}$ (c'est la conique plane si $m=2$ ). Remarquons que le groupe fondamental local de la singularité du revêtement universel est ici trivial.

Théorème 11.18 Soit $(X \mid \Delta)$ lisse, avec $X$ complexe, compacte et connexe. Si $\pi_{1}(X \mid \Delta)$ est résiduellement fini, il existe un revêtement fini ramifié en $\Delta$ (exactement) $g: \bar{X} \rightarrow(X \mid \Delta)$ tel que $\pi_{1}\left(\bar{X}^{0}\right) \rightarrow \pi_{1}(\bar{X})$ soit bijectif, et $\pi_{1}(\tilde{X})$ est un sous-goupe d'indice fini de $\pi_{1}(X \mid \Delta)$, si $\sigma: \tilde{X} \rightarrow \bar{X}$ est une résolution des singularités (quotient) de $\bar{X}$.

Démonstration : On peut naturellement stratifier $\operatorname{Supp}(\Delta)$ comme réunion disjointe d'un nombre fini de sous-variétés lisses $V_{j}$, localement 
fermées. A chacune des $V_{j}$ est attaché un sous-groupe fini abélien $G_{j}$ de $\pi_{1}(X \mid \Delta)$, bien défini à conjugaison près seulement, et égal au groupe fondamental local de $(X \mid \Delta)$ en un point quelconque de $V_{j}$. Le groupe $\pi_{1}(X \mid \Delta)$ étant supposé résiduellement fini, il admet un sous-groupe normal d'indice fini qui ne rencontre qu'en $\{1\}$ chacun des $G_{j}$. Le revêtement associé à un tel sous-groupe satisfait les conditions énoncées, puisque les groupes fondamentaux locaux deviennent tous triviaux sur un tel revêtement, donc $\pi_{1}\left(\bar{X}_{0}\right)=\pi_{1}(\bar{X})$.

Théorème 11.19 Soit $(X \mid \Delta)$ une orbifolde géométrique lisse, avec $X \in \mathcal{C}$, connexe. Si $\pi_{1}(X \mid \Delta)$ est résoluble et résiduellement fini ${ }^{39}$, alors $\pi_{1}(X \mid \Delta)$ est presque nilpotent.

Si $(X \mid \Delta)$ est spéciale, et si $\pi_{1}(X \mid \Delta)$ est linéaire (ie : plongeable dans un $G l(N, \mathbb{C}))$, alors $\pi_{1}(X \mid \Delta)$ est presque abélien.

Démonstration : Soit $\bar{X}, \tilde{X}$ comme dans 11.18 ci-dessus (puisque $\pi_{1}(X \mid \Delta)$ est résiduellement fini, de tels revêtements existent). On a donc $\tilde{X} \in \mathcal{C}$, avec $\pi_{1}(\tilde{X})$ résoluble. Par [De06], $\pi_{1}(\tilde{X})$ est donc presque nilpotent. $\operatorname{Par} 11.18, \pi_{1}(\tilde{X})$ est d'indice fini dans $\pi_{1}(X \mid \Delta)$. Ce dernier groupe est donc presque-nilpotent.

La seconde assertion résulte de ce qui précède et de [Ca04, theorem 7.8], puisque $\tilde{X}$ est spéciale.

Théorème $11.20{ }^{40}$ Soit $X \in \mathcal{C}$ telle que, pour tout revêtement fini étale $X^{\prime}$ de $X$, l'application d'Albanese de $X^{\prime}$ soit surjective. Alors tout groupe quotient $G$ de $\pi_{1}(X)$ qui est résoluble est presque abélien.

En particulier, si $X$ est spéciale, tout quotient résoluble de $\pi_{1}(X)$ est presque abélien.

Démonstration : Il résulte de [Delz06] que $G$ est presque nilpotent (sinon un revêtement étale fini de $X$ fibre sur une courbe de genre 2 ou plus, contredisant l'hypothèse sur l'application d'Albanese). Il résulte alors de [Ca95] que $G$ est presque abélien.

Par les mêmes arguments que ceux de 11.19, on déduit de 11.20 :

\footnotetext{
${ }^{39} \mathrm{Il}$ existe des groupes résolubles de présentation finie non résiduellement finis : voir O. Kharlampovich. Izv. Akad. Nauk 45 (1981), p. 852. Je remercie T. Delzant pour cette référence.

${ }^{40}$ Ce résultat est inspiré par une discussion avec K. Yamanoi.
} 
Corollaire 11.21 Si $(X \mid \Delta)$ est lisse et spéciale, avec $X \in \mathcal{C}$, et si $\pi_{1}(X \mid \Delta)$ est résiduellement fini, tout quotient résoluble de $\pi_{1}(X \mid \Delta)$ est presque abélien.

Remarque 11.22 L'hypothèse de finitude résiduelle de 11.19 est probablement superflue dans 11.19 et 11.21.

\section{5 $\Gamma$-réduction (ou réduction de Shafarevich) orbi- folde.}

Nous étendons partiellement au cadre orbifolde géométrique certains résultats de [Ca94] :

Soit $(X \mid \Delta)$ une orbifolde géométrique lisse, et $V \subset X$ Zariski-fermé irréductible, non contenu dans le support de $\Delta$. Soit alors $\left(V \mid \Delta_{V}\right)$ une restriction divisible ${ }^{41}$ de $\Delta$ à $V$, au sens de 2.38. Cette restriction induit, par 11.3, un morphisme de groupes $\pi_{1}\left(V \mid \Delta_{V}\right) \rightarrow \pi_{1}(X \mid \Delta)$ dont l'image est bien définie à conjugaison près dans $\pi_{1}(X \mid \Delta)$, et est indépendante de la restriction (divisible) $\left(V \mid \Delta_{V}\right)$ choisie (c'en est même un invariant biméromorphe).

On notera $\pi_{1}\left(V \mid \Delta_{V}\right)_{(X \mid \Delta)}$ cette image. C'est un quotient de $\pi_{1}\left(V \mid \Delta_{V}\right)$.

Exemple 11.23 Soit $R \subset(X \mid \Delta)$ une courbe rationnelle orbifolde. Alors $\pi_{1}\left(R \mid \Delta_{R}\right)_{(X \mid \Delta)}$ est un groupe fini, puisque $(X \mid \Delta)$ admet pour revêtement universel $\left(\mathbb{P}^{1} \mid \Delta^{\prime}\right)$, avec $\Delta^{\prime}$ vide ou supportée par un seul point.

Nous allons maintenant introduire une version orbifolde de la $\Gamma$-réduction de [Ca94] (voir aussi [Ko93] dans le cas projectif).

Théorème 11.24 Soit $(X \mid \Delta)$ une orbifolde géométrique lisse, avec $X \in \mathcal{C}$. Il existe une unique fibration méromorphe presque holomorphe $g: X \rightarrow Y$ telle que :

1. Pour $y \in Y$ générique, $\pi_{1}\left(X_{y} \mid \Delta_{X_{y}}\right)_{(X \mid \Delta)}$ est fini.

2. Pour $y \in Y$ général, si $V \subset X$ est Zariski-fermé irréductible tel que $V$ rencontre $X_{y}$, et si $\pi_{1}\left(V \mid \Delta_{V}\right)_{(X \mid \Delta)}$ est fini, alors $V \subset X_{y}$.

La fibration $g$ est appelée la $\Gamma$-réduction (ou réduction de Shafarevich) de $(X \mid \Delta)$. On la note : $\gamma_{(X \mid \Delta)}:(X \mid \Delta) \rightarrow \Gamma(X \mid \Delta)$. La dimension $\operatorname{dim}(Y)$

\footnotetext{
${ }^{41}$ C'est-à-dire que les morphismes orbifoldes de cette définition sont cette fois-ci, divisibles.
} 
de la base de cette réduction est appelée la $\Gamma$-dimension de $(X \mid \Delta)$, notée $\gamma d(X \mid \Delta)$.

On dit que $(X \mid \Delta)$ est de $\pi_{1}$-type général $\operatorname{sidim}(Y)=\operatorname{dim}(X)$ ci-dessus.

Démonstration : Notations du §.5.12. Soit $A \subset \mathcal{C}(X)$ le sous-ensemble des $a$ tels que $Z_{a}$ soit (réduit), non contenu dans $\operatorname{Supp}(\Delta)$, et $\pi_{1}\left(V \mid \Delta_{V}\right)_{(X \mid \Delta)}$ fini. Il suffit, d'après $5.57,5.58$ et 5.60 , pour établir l'assertion, de montrer que $A \subset \mathcal{C}(X)$ est Z-régulier et stable. Soit donc $B \subset \mathcal{C}(X)$ analytique fermé irréductible, donc compact. Supposons que $A \cap B$ ne soit pas contenu dans une réunion dénombrable de sous-ensembles Zariski fermés stricts de $B$. Nous allons montrer que $A \cap B$ contient le point générique de $B$. Soit $Z \subset B \times X$ le graphe d'incidence (normalisé) de la famille de cycles $\left\{Z_{b}, b \in B\right\}$ paramétrée par $B$. Par hypothèse, $Z$ est irréductible, non contenu dans $B \times D$, $D:=\operatorname{supp}(\Delta)$. Soit $X^{\prime} \subset X$ la réunion (Zariski fermée) des $Z_{b}$. Par une modification lisse adéquate $\mu: Y^{\prime} \rightarrow Y:=B \times X$, on peut supposer que $X^{\prime}$ (ou plutoôt sa transformée stricte dans $\left.Y^{\prime}\right)$ rencontre transversalement $\Delta^{\prime}$, diviseur orbifolde sur $Y^{\prime}$ tel que $\left(Y^{\prime} \mid \Delta^{\prime}\right)$ soit lisse, et que $\mu:\left(Y^{\prime} \mid \Delta^{\prime}\right) \rightarrow(Y / B \times \Delta)$ soit biméromorphe. On suppose aussi que $X^{\prime}$ rencontre transversalement $B \times \Delta$. De sorte que $\left(X^{\prime} \mid \Delta_{X^{\prime}}^{\prime}\right)$ est une restriction de $(B \times X / B \times \Delta)$ à $X^{\prime}$. Cette restriction induit, par restriction à $Z_{b}, b \in B$ générique, une restriction de $\Delta$ à $Z_{b}$. Il nous suffit de montrer que $\pi_{1}\left(Z_{b} \mid \Delta_{Z_{b}}\right)_{(X \mid \Delta)}$ est indépendant de $b \in B$ générique. Ceci est maintenant évident, puisque l'on a une factorisation : $\pi_{1}\left(Z_{b} \mid \Delta_{Z_{b}}\right) \rightarrow \pi_{1}\left(X^{\prime} \mid \Delta_{X^{\prime}}^{\prime}\right) \rightarrow \pi_{1}(X \mid \Delta)$, et que l'image de la première flèche est indépendante de $b \in B$, générique (tel que sa restriction, vue dans $\left(X^{\prime} \mid \Delta_{X^{\prime}}^{\prime}\right)$, soit une orbifolde géométrique lisse). QED pour la Z-régularité. Nous considérons maintenant la stabilité de $A$.

Quitte à construire, comme auparavant, des modifications adéquates, il suffit de voir que si $g: V \rightarrow W$ est une fibration, avec $W, V \in \mathcal{C}$ lisses et connexes, et $h: V \rightarrow X$ biméromorphe sur son image $V^{\prime}:=h(V)$, alors $V^{\prime} \in A$ si $g\left(V_{w}\right) \in A$, et s'il existe $Z \subset V$ analytique fermé irréductible tel que $g(Z)=W$ et $h(Z) \in A$. Soit donc (ce que l'on peut supposer) $\left(V \mid \Delta_{V}\right)$ une restriction (divisible) de $\Delta$ à $V^{\prime}$ telle que $g: V \rightarrow W$ soit nette. On a donc une une suite exacte de groupes fondamentaux orbifoldes :

$$
\pi_{1}\left(V_{w} \mid \Delta_{V \mid V_{w}}\right) \longrightarrow \pi_{1}\left(V \mid \Delta_{V}\right) \longrightarrow \pi_{1}\left(W \mid \Delta_{W}\right) \longrightarrow\{1\}
$$

Dans laquelle $\left(W \mid \Delta_{W}\right)$ est la base orbifolde de $g:\left(V \mid \Delta_{V}\right) \rightarrow W$. D'après 11.3, on a un morphisme d'image d'indice fini : $\pi_{1}\left(Z \mid \Delta_{Z}\right) \rightarrow \pi_{1}\left(W \mid \Delta_{W}\right)$.

Prenant les images par $h_{*}$ dans $\pi_{1}(X \mid \Delta)$, on en déduit que $G:=$ 
$h_{*}\left(\pi_{1}\left(V \mid \Delta_{V}\right)\right)$ admet $N:=h_{*}\left(\pi_{1}\left(V_{w} \mid \Delta_{V \mid V_{w}}\right)\right)$ comme sous-groupe normal, le quotient admettant un sous-groupe d'indice fini quotient de $Q:=$ $h_{*}\left(\pi_{1}\left(Z \mid \Delta_{Z}\right)\right)$. Par hypothèse, $N$ et $Q$ sont finis, donc aussi $G$.

Corollaire 11.25 Soit $(X \mid \Delta)$ une orbifolde géométrique lisse rationnellement engendrée (ie : RE). Alors $\pi_{1}(X \mid \Delta)$ est fini.

Démonstration : Puisque $(X \mid \Delta)$ est une tour de fibrations à fibres orbifoldes RCC, il suffit, par 5.26 et 5.23 , de montrer le résultat lorsque $(X \mid \Delta)$ est RCC. Puisque deux points génériques de $X$ sont alors joints par une chaine connexe de courbes orbifoldes dont les groupes fondamentaux sont finis, l'assertion résulte de 11.24

Remarque 11.26 Il est possible de construire (comme dans [Ca94]) la $\tilde{\Gamma}$ réduction d'un revêtement orbifolde étale (non compact). La construction de [Ca94] nécessite cependant, pour être adaptée, la construction d'une métrique de Kähler adéquate sur ce revêtement, et l'élaboration d'arguments techniquement différents de ceux présentés ici. ${ }^{42}$

Rappelons ([Ca98]) :

Definition 11.27 Une classe $\mathcal{G}$ de groupes est dite stable si :

1. Tout groupe isomorphe à $G \in \mathcal{G}$ est dans $\mathcal{G}$.

2. Tout quotient, tout sous-groupe d'indice fini de $G \in \mathcal{G}$ est dans $\mathcal{G}$.

3. Tout extension de deux groupes de $\mathcal{G}$ est dans $\mathcal{G}$.

Les exemples les plus simples sont les classes des groupes finis, virtuellement (ou presque) abéliens, virtuellement polycycliques, virtuellement résolubles. La classe des groupes virtuellement nilpotents n'est pas stable.

Corollaire 11.28 Soit $(X \mid \Delta)$ une orbifolde géométrique lisse, avec $X \in \mathcal{C}$ connexe. Soit $\mathcal{G}$ une classe stable de groupes. Si deux points génériques de $X$ peuvent être joints par une chaine connexe de sous-variétés (analytiques fermées irréductibles) $V \subset X$ telles que $\pi_{1}\left(V \mid \Delta_{V}\right)_{(X \mid \Delta)} \in \mathcal{G}$, alors $\pi_{1}(X \mid \Delta) \in$ $\mathcal{G}$.

\footnotetext{
${ }^{42}$ Cette construction a été récemment faite par B. Claudon dans [Cla08].
} 
Démonstration : Lorsque $\mathcal{G}$ est la classe des groupes finis, c'est une conséquence immédiate de 11.24. En général, on considère le sous-ensemble $A \in \mathcal{C}(X)$ constitué des $a$ tels que $\pi_{1}\left(Z_{a} \mid \Delta_{Z_{a}}\right)_{(X \mid \Delta)} \in \mathcal{G}$. Il s'agit de montrer que cet ensemble est Z-régulier et stable. Les arguments sont les mêmes que ceux de la démonstration de $11.24^{43}$, et sont exposés dans [Ca98] et [Ca04] auxquels nous renvoyons.

\footnotetext{
${ }^{43} \mathrm{Il}$ suffit, dans la première (resp. dernière) ligne, de remplacer : " $\pi_{1}\left(V \mid \Delta_{V}\right)_{(X \mid \Delta)}$ fini" par : " $\pi_{1}\left(V \mid \Delta_{V}\right)_{(X \mid \Delta)} \in \mathcal{G}$ " (resp. " $N, Q$ finis" par : " $N, Q \in \mathcal{G}$ ")
} 


\section{CONJECTURES}

Les conjectures qui suivent sont motivées par le lemme de dévissage 10.12 dans le cas des orbifoldes géométriques spéciales, et par des conjectures standard dans le cas des variétés (lisses) avec $\kappa=0$ ou $\kappa_{+}=-\infty$ (conjecturalement rationnellement connexes). D'autres sont des versions orbifoldes de celles de S. Lang (voir [La86]) concernant l'arithmétique et l'hyperbolicité des variétés de type général. Ces conjectures orbifoldes semblent être des intermédiaires incontournables pour atteindre les propriétés conjecturales des variétés spéciales (sans structure orbifolde), puis des variétés arbitraires, à l'aide du "coeur".

Nous ne cherchons pas à établir une liste (pourtant limitée) des cas connus.

\subsection{Stabilité par déformation et spécialisation}

Definition 12.1 Soit $0 \in S$ un espace analytique connexe pointé, et $(X \mid \Delta)$ une orbifolde géométrique lisse, avec $X \in \mathcal{C}$. Une déformation de $(X \mid \Delta)$ paramétrée par $S$ est une orbifolde géométrique lisse $(\mathcal{X} \mid \mathcal{D})$ munie d'une submersion surjective propre et connexe $f: \mathcal{X} \rightarrow S$ dont toutes les fibres sont dans $\mathcal{C}$, et telle que :

1. Si $\mathcal{D}=\sum_{j}\left(1-\frac{1}{m_{j}}\right) . \mathcal{D}_{j}$, alors chacune des $\mathcal{D}_{j}$ est lisse, et la restriction de $f \grave{a} \mathcal{D}_{j}$ est submersive.

2. $\left(\mathcal{X}_{0} / \mathcal{D}_{0}\right)=(X \mid \Delta)$, posant : $\Delta_{s}:=\sum_{j}\left(1-\frac{1}{m_{j}}\right) \cdot\left(\mathcal{D}_{j}\right)_{s}, \forall s \in S$.

Conjecture 12.2 La dimension essentielle de $(X \mid \Delta)$ est invariante par déformation (ie : $\forall s \in S$,ess $\left(X_{s} \mid \Delta_{s}\right)=\operatorname{ess}\left(X_{0} \mid \Delta_{0}\right)$ dans la situation précédente). En particulier, si $\left(X_{0} \mid \Delta_{0}\right)$ est spéciale, $\forall s \in S,\left(X_{s} \mid \Delta_{s}\right)$ est spéciale.

Plus précisément : il existe une fibration méromorphe (unique) $c: \mathcal{X} \rightarrow$ $\mathcal{C}$ au-dessus de $S$ qui induit, pour tout $s \in S$, une fibration méromorphe $c_{s}: X_{s} \rightarrow C_{s}$ qui est le coeur de $\left(X_{s} \mid \Delta_{s}\right)$. Sur un modèle biméromorphe adéquat, la base orbifolde stable de $(\mathcal{C} / \mathcal{D}(c, \Delta))$ est une déformation de celle de $\left(X_{0} \mid \Delta_{0}\right)$.

L'algèbre essentielle $A(X \mid \Delta)$ est aussi un invariant de déformation. Elle est de type fini. (Voir 9.1 et 2.16 pour sa définition).

Les deux dernières conjectures sont analogues à l'invariance par 
déformation de l'algèbre canonique, et à la finitude de ses générateurs ([Siu01], [Pa05], [BCHM06]).

Nous allons maintenant voir que ces conjectures peuvent être réduites, dans une certaine mesure, par le dévissage de §.10.5, au cas de l'invariance par déformation des plurigenres en version orbifolde, et au cas $\kappa_{+}=-\infty$. Ce dévissage suggérant l'invariance par déformation de nouveaux invariants intermédiaires définissant le type (voir définition 10.6), et en particulier de la longueur $\nu(X \mid \Delta)$. Le lemme 10.12 montre en effet immédiatement que les conjectures 12.2 résultent des suivantes (et de $C_{n, m}^{o r b}$, ou de sa conséquence : l'existence du $\kappa$-quotient rationnel) :

Conjecture 12.3 1. $\kappa(X \mid \Delta)$ est invariante par déformation (ie : $\forall s \in S$, $\kappa\left(X_{s} \mid \Delta_{s}\right)=\kappa\left(X_{0} \mid \Delta_{0}\right)$ dans la situation précédente). L'algèbre canonique $K(X \mid \Delta)$ est de type fini, et invariante par déformation. (Voir 2.16 pour la définition).

2. La dimension du $\kappa$-quotient rationnel $r_{(X \mid \Delta)}^{+}$de $(X \mid \Delta)$ est un invariant de déformation.

3. Si $\kappa(X \mid \Delta) \geq 0$, il existe une fibration méromorphe (unique) $\mu: \mathcal{X} \rightarrow$ $\mathcal{M}$ au-dessus de $S$ qui induit, pour tout $s \in S$, une fibration méromorphe $\mu_{s}: X_{s} \rightarrow M_{s}$ qui est la fibration de Moishezon-Iitaka de $\left(X_{s} \mid \Delta_{s}\right)$. Sur un modèle biméromorphe adéquat, la base orbifolde stable de $(\mathcal{M} / \mathcal{D}(\mu, \Delta))$ est une déformation de celle de $\left(X_{0} \mid \Delta_{0}\right)$.

4. il existe une fibration méromorphe (unique) $r: \mathcal{X} \rightarrow \mathcal{R}$ au-dessus de $S$ qui induit, pour tout $s \in S$, une fibration méromorphe $r_{s}^{+}: X_{s} \rightarrow R_{s}^{+}$qui est le $\kappa$-quotient rationnel de $\left(X_{s} \mid \Delta_{s}\right)$. Sur un modèle biméromorphe adéquat, la base orbifolde stable de $(\mathcal{R} / \mathcal{D}(r, \Delta))$ est une déformation de celle de $\left(X_{0} \mid \Delta_{0}\right)$.

Remarque 12.4 1. Les résultats de [Cla06] et de [BCHM06] établissent respectivement la stabilité par déformation de $K(X \mid \Delta)$ et la finitude de son type (du moins lorsque $\Delta=0$ ). La conjecture 5.48 implique facilement l'invariance par déformation du $\kappa$-quotient rationnel.

2. Les conditions imposées aux composantes de $\mathcal{D}$ dans la définition 12.1 semblent nécessaires. Dans l'exemple (logarithmique)suivant, où $\mathcal{D}$ est lisse, mais $f_{\mathcal{D}}$ non submersive, le quotient rationnel est trivial pour $s \neq 0$, mais non-trivial pour $s=0: X_{s}=\mathbb{P}^{2}, \forall s$, et $\Delta_{s}$ est une conique lisse (réduite) si $s \neq 0$, et la réunion de 2 droites si $s=0$. Cette pathologie semble cependant liée à la singularité log-canonique non klt qui apparait sur la fibre centrale. 
Exemple 12.5 (Suggéré par une remarque de $Y$. Tschinkel). Soit $X_{0}^{\prime}$ le cône sur une hypersurface lisse de degré $(n+2)$ (donc de type général) de $\mathbb{P}_{n}, n \geq 2$. Il est spécialisation d'une famille d'hypersurfaces lisses de degré $(n+2)($ donc spéciales) de $\mathbb{P}_{n+1}$. Soit $X_{0}$ le transformé total de $X_{0}^{\prime}$ dans l'éclaté de $\mathbb{P}_{n+1}$ en le sommet de $X_{0}^{\prime}$. Donc $X_{0}$ a deux composantes : l'éclaté de $X_{0}^{\prime}$ en son sommet, qui n'est pas spécial, et une seconde composante isomorphe à $\mathbb{P}_{n}$. Et $X_{0}$ est encore spécialisation de la famille précédente de variétés spéciales. Observons que $X_{0}$ est $\mathcal{S}$-connexe (avec la définition 12.6 ci-dessous), bien que l'une de ses composantes ne le soit pas.

Definition 12.6 Une orbifolde géométrique $(X \mid \Delta)$ est dite $\mathcal{S}$-connexe si $X \in \mathcal{C}$ est de dimension pure, si ses composantes irréductibles sont lisses et se coupent transversalement, si chaque composante $\left(X_{k} \mid \Delta_{k}\right)$ de $(X \mid \Delta)$ est lisse, et si deux points génériques de $X$ peuvent être joints par une chaine connexe de sous-orbifoldes géométriques spéciales.

Conjecture 12.7 Toute spécialisation d'orbifoldes géométriques lisses spéciales est $\mathcal{S}$-connexe.

Cette conjecture ne semble pas pouvoir être simplement déduite par dévissage des cas $\kappa=0$ et $\kappa_{+}=-\infty$.

Dans une toute autre direction :

Conjecture $12.8 \gamma d(X \mid \Delta)$ est invariante par déformation.

Le cas des familles projectives de variétés de dimension 3 (sans structure orbifolde géométrique) a été partiellement résolu par B. Claudon [Cla07].

\subsection{Groupe fondamental et revêtement universel}

Conjecture 12.9 Soit $(X \mid \Delta)$ une orbifolde géométrique lisse entière, avec $X \in \mathcal{C}$ connexe.

1. Si $(X \mid \Delta)$ est spéciale, alors $\pi_{1}(X \mid \Delta)$ est presque abélien (noté : $\pi_{1}(X \mid \Delta) \in \widetilde{A b}$, ceci signifie que ce groupe a un sous-groupe d'indice fini abélien). En particulier :

2. Si $\kappa(X \mid \Delta)=0$, alors $\pi_{1}(X \mid \Delta) \in \widetilde{A b}$.

3. Si $\kappa_{+}(X \mid \Delta)=-\infty$, alors $\pi_{1}(X \mid \Delta)$ est fini. 
Dans ce cas particulier, le lemme de "dévissage" 10.12 permet de réduire la conjecture 12.9.(1) à ses cas particuliers 12.9.(2) et (3).

Proposition 12.10 Supposons la conjecture $C_{n, m}^{o r b}$ vraie, ainsi que les énoncés (2) et (3) de la conjecture 12.9. Alors l'énoncé (1) de la conjecture 12.9 est également vrai.

Démonstration : Il suffit de montrer que si $f:(X \mid \Delta) \rightarrow Y$ est une fibration dont la base orbifolde stable et la fibre orbifolde générique ont un $\pi_{1}$ presque abélien, alors $G:=\pi_{1}(X \mid \Delta) \in \widetilde{A b}$ aussi. Or ce groupe est extension d'un groupe presque abélien de type fini par un autre groupe du même type. Donc $G$ est polycyclique, et en particulier résoluble linéaire, et donc résiduellement fini. Il résulte donc de 11.19 que $G$ est presque presque-abélien.

Remarque 12.11 0. On a montré en 11.19 que la conjecture 12.9(1) est vraie si $\pi_{1}(X \mid \Delta)$ est supposé linéaire.

1. Le cas particulier de 12.9(3) où $(X \mid \Delta)$ est Fano (ie : $-K_{(X \mid \Delta)}$ ample) est peut-être accessible par les méthodes $L^{2}$, ou la construction de métriques orbifoldes à courbure de Ricci positive lorsque es multiplicités sont finies. (Dans le cas logarithmique, la condition $(X \mid \Delta)$ Fano n'implique en effet pas que : $\left.\kappa_{+}(X \mid \Delta)=-\infty\right)$.

2. Le cas particulier où $K_{(X \mid \Delta)} \equiv 0$ est peut-être accessible par l'usage de métriques de Kähler-Einstein. (Voir [Ca02] pour le cas très particulier des variétés à singularités quotient).

3. Lorsque $(X \mid \Delta)$ est $R E$, la conjecture 12.9.(3) est vraie, par 11.25. La conjecture 12.9.(3) est donc une conséquence de 11.25, et de la conjecture 5.48 .

Question 12.12 Soit $(X \mid \Delta)$ une orbifolde géométrique lisse, avec $X \in \mathcal{C}$ connexe.

1. Existe-t-il $Y \in \mathcal{C}$, normal, tel que $\pi_{1}(Y)$ et $\pi_{1}(X \mid \Delta)$ soient commensurables (ie : admettent des sous-groupes d'indices finis isomorphes)?

2. Existe-t-il $Z \in \mathcal{C}$, normal, tel que les revêtements universels de $Z$ et de $(X \mid \Delta)$ soient analytiquement isomorphes?

Lorsque $(X \mid \Delta)$ admet un revêtement étale fini $\bar{X} \rightarrow(X \mid \Delta)$ comme en 11.16, et en particulier lorsque $\pi_{1}(X \mid \Delta)$ est résiduellement fini, la réponse à ces deux questions est "oui", avec $Z=Y=\bar{X}$. 


\subsection{Pseudométrique de Kobayashi}

On rappelle la notion de pseudométrique de Kobayashi d'une orbifolde géométrique $(X \mid \Delta)$ : c'est la plus grande des pseudométriques $d: X \times X \rightarrow$ $\left[0,+\infty\left[\right.\right.$ telles que $d \leq h^{*}\left(d_{\mathbb{D}}\right)$, pour tout morphisme orbifolde $h: \mathbb{D} \rightarrow(X \mid \Delta)$, $d_{\mathbb{D}}$ étant la métrique de Poincaré sur le disque unité $\mathbb{D}$. On la note $d_{(X \mid \Delta)}$. Considérant seulement les morphismes orbifoldes divisibles : $\mathbb{D} \rightarrow(X \mid \Delta)$, on obtient la pseudométrique classique (ou divisible) $d_{(X \mid \Delta)}^{*}$. On a $: d_{(X \mid \Delta)} \leq$ $d_{(X \mid \Delta)}^{*}$.

Conjecture 12.13 Soit $(X \mid \Delta)$ une orbifolde géométrique lisse, avec $X \in \mathcal{C}$, connexe.

1. $d_{(X \mid \Delta)}$ est nulle si et seulement si $(X \mid \Delta)$ est spéciale.

2. Si $(X \mid \Delta)$ est de type général, il existe un ouvert de Zariski dense $U$ de $X$ tel que la restriction de $d_{(X \mid \Delta)}$ à $U \times U$ soit une métrique.

3. Si c : $(X \mid \Delta) \rightarrow C(X \mid \Delta)$ est le coeur, alors $d_{(X \mid \Delta)}=c^{*}\left(d_{(C(X \mid \Delta) \mid \Delta(c, \Delta))}\right)$.

\section{Remarque $\mathbf{1 2 . 1 4}$}

1. La conjecture (1) précédente peut être réduite aux cas $\kappa=0$ et $\kappa^{+}=$ $-\infty$ par dévissage si l'on peut montrer qu'une orbifolde géométrique munie d'une fibration a une pseudométrique de Kobayashi nulle s'il en est de même pour sa base et ses fibres orbifoldes.

2. L'assertion (2) est une version orbifolde de la conjecture hyperbolique de S. Lang.

3. L'assertion (3) signifie qu'il n'y a pas d'obstruction globale au relèvement à $(X \mid \Delta)$ des morphismes orbifoldes $h: \mathbb{D} \rightarrow(C(X \mid \Delta) \mid \Delta(c, \Delta))$, base orbifolde du coeur.

4. La conjecture 12.13(1) est établie pour les courbes dans [C-W05], où l'on montre aussi que le lemme de Brody reste valable en version orbifolde géométrique.Dans [C-P 05], une version orbifolde des théorèmes d'hyperbolicité de Bogomolov et $\mathrm{M}^{c}$ Quillan est établie pour certaines surfaces orbifoldes avec $\left(c_{1}^{2}-c_{2}\right)>0$. Voir [R 08] pour de nombreux compléments intéressants et améliorations sur ce sujet.

On peut tenter de relier l'annulation de la pseudométrique de Kobayashi orbifolde à l'existence de courbes entières orbifoldes. 
Conjecture 12.15 Soit $(X \mid \Delta)$ une orbifolde lisse, avec $X \in \mathcal{C}$. On a équivalence entre les propriétés suivantes:

1. $(X \mid \Delta)$ est spéciale.

2. $d_{(X \mid \Delta)} \equiv 0$.

3. Il existe une $\Delta^{\text {div }}$-courbe entière (ie : un morphisme $h: \mathbb{C} \rightarrow(X \mid \Delta)^{\text {div }}$ ) dont l'image est Zariski-dense dans $X$.

4. Il existe une $\Delta^{\text {div }}$-courbe entière dont l'image est dense (pour la topologie analytique) dans $X$.

5. Tout sous-ensemble fini de $X$ est contenu dans une $\Delta^{\text {div }}$-courbe entière dont l'image est dense dans $X$.

\section{Exemple 12.16}

1. Soit $X$ une variété quasi-projective lisse, et $X=(\bar{X}-D)$ une compactification de $X$ lisse telle que $D$ soit un diviseur à croisements normaux de $\bar{X}$. On dit que $X$ est spéciale si $(\bar{X} \mid D)$ est spéciale. Cette définition ne dépend pas de la compactification choisie. La conjecture précedente affirme donc en particulier que $X$ est spéciale si et seulement si elle contient une courbe entière (au sens usuel) Zariski-dense.

2. Un récent (Novembre 2008) preprint de J. Winkelmann caractérise les surfaces quasi-projectives $X$ dont l'application quasi-Albanese a une image de dimension 2, et qui contiennent une courbe entière Zariski-dense. Sa caractérisation semble coincider dans ce cas avec le fait d'être spéciale, et donc fournir pour cette classe de surfaces une solution (positive) de la conjecture précédente.

3. Si $(X \mid \Delta)$ lisse, entière, avec $X \in \mathcal{C}$ contient une $\Delta^{\text {div }}$-courbe entière Zariski-dense, elle est d'après la conjecture précédente, spéciale, et son groupe fondamental est donc presque-abélien. En particulier, une variété quasi-projective contenant une courbe entière (au sens usuel) Zariski-dense a un groupe fondamental presque-abélien.

\subsection{Points rationnels : corps de fonctions}

Soit $B$ une courbe projective complexe lisse et connexe, et $k_{B}:=\mathbb{C}(B)$ le corps de ses fonctions méromorphes. Soit $f: X \rightarrow B$ une application holomorphe surjective et connexe, $X$ étant une variété projective complexe lisse (et connexe, donc). Pour $b \in B$, on note $X_{b}$ la fibre schématique de $f$ audessus de $b$. On suppose $X$ muni d'une structure d'orbifolde géométrique lisse $(X \mid \Delta)$ qui induit, pour $b \in B$ générique, une structure d'orbifolde 
géométrique lisse $\left(X_{b} \mid \Delta_{b}\right)=(X \mid \Delta)_{b}$ sur $X_{b}$. On dira simplement que $(X \mid \Delta)$ est une orbifolde géométrique lisse définie sur $k_{B}$. On dira que $(X \mid \Delta)$ est spéciale (resp. de type général, etc...) s'il en est de même pour $\left(X_{b} \mid \Delta_{b}\right)$, pour $b \in B$ général. Voir [Ca 01] pour plus de détails.

Un point $k_{B}$-rationnel $s$ de $X$ sur $k_{B}$ est une section $s: B \rightarrow X$ de $f$. On note $X\left(k_{B}\right)$ l'ensemble de ces points. (Cet ensemble est essentiellement un invariant birationnel de $(X, f)$ : si $\left(X^{\prime}, f^{\prime}\right)$ est un second modèle birationnel de $f, X\left(k_{B}\right)$ et $X^{\prime}\left(k_{B}\right)$ coincident sur un ouvert de Zariski non vide commun).

Soit $S \subset B$ un sous-ensemble fini au dessus du complémentaire duquel $(X \mid \Delta)$ a bonne réduction, c'est-à-dire est tel que $\left(X_{b} \mid \Delta_{b}\right)$ est lisse si $b \notin S$.

Si $\Delta=\sum_{j \in J}\left(1-1 / m_{j}\right) D_{j}$, si $s \in X\left(k_{B}\right)$, si $b \notin S$, et si $j \in J$, on note $\left(s . D_{j}\right)_{b}$ l'ordre de contact en $s(b)$ de $s(B)$ avec $D_{j}$. C'est un entier positif (ou nul).

On définit alors $(X \mid \Delta)\left(k_{B}, S\right)$ comme le sous-ensemble des $s \in X\left(k_{B}\right)$ tels que $\forall j, b \notin S$, on ait : $\left(s . D_{j}\right)_{b} \geq m_{j}$ si $\left(s . D_{j}\right)_{b} \geq 1$. Autrement dit : l'ordre de contact doit être au moins égal à $m_{j}$ si $s(b) \in D_{j}$, ceci pour tous $j \in J, b \notin S$.

On note $(X \mid \Delta)\left(k_{B}\right)$ la réunion des $(X \mid \Delta)\left(k_{B}, S^{\prime}\right)$, lorsque $S^{\prime} \subset B$ est finie, de complémentaire de bonne réduction au sens précédent. Cet ensemble est essentiellement un invariant birationnel de $((X \mid \Delta), f)$.

On peut définir ces notions en version classique: $(X \mid \Delta)^{*}\left(k_{B}, S\right)$ est alors le sous-ensemble des $s \in X\left(k_{B}\right)$ tels que $\forall j, b \notin S$, on ait : $\left(s . D_{j}\right)_{b}$ est divisible par $m_{j}$. On a, bien sûr : $(X \mid \Delta)^{*}\left(k_{B}, S\right) \subset(X \mid \Delta)\left(k_{B}, S\right)$.

Les extensions finies de corps $k^{\prime} / k$ correspondent bijectivement aux morphismes finis $B^{\prime} \rightarrow B, B^{\prime}$ courbe projective lisse et connexe, posant : $k^{\prime}=k_{B^{\prime}}$. Un morphisme $f:(X \mid \Delta) \rightarrow B$ comme ci-dessus définit alors par changement de base (et désingularisation) un morphisme $f^{\prime}:\left(X^{\prime} \mid \Delta^{\prime}\right) \rightarrow B^{\prime}$, et des inclusions $(X \mid \Delta)\left(k_{B}, S\right) \subset(X \mid \Delta)\left(k_{B^{\prime}}, S^{\prime}\right)$, avec $S^{\prime}$ image inverse de $S$ dans $B^{\prime}$.

Si $g:(X \mid \Delta) \rightarrow\left(Y \mid \Delta_{Y}\right)$ est un morphisme orbifolde au-dessus de $B$, il induit naturellement une application $f:(X \mid \Delta)\left(k_{B}, S\right) \rightarrow\left(Y \mid \Delta_{Y}\right)\left(k_{B}, S\right), \forall S$. Si $f$ est un morphisme orbifolde divisible (ou classique), il induit de même une application $f:(X \mid \Delta)^{*}\left(k_{B}, S\right) \rightarrow\left(Y \mid \Delta_{Y}\right)^{*}\left(k_{B}, S\right)$.

On dit que $f:\left(Y \mid \Delta_{Y}\right) \rightarrow B$ est isotrivial s'il existe $B^{\prime} \rightarrow B$ fini, et $\left(F \mid \Delta_{F}\right)$ une orbifolde géométrique lisse telle que $\left(Y \mid \Delta_{Y}\right) \times_{B} B^{\prime}$ soit birationnel au-dessus de $B^{\prime}$ à $\left(F \mid \Delta_{F}\right) \times B^{\prime}$.

On dit que $(X \mid \Delta)$ n'a pas de quotient isotrivial ( $\left.\operatorname{sur} k_{B}\right)$ s'il n'existe pas de morphisme orbifolde méromorphe $g:(X \mid \Delta) \rightarrow\left(Y \mid \Delta_{Y}\right)$ dominant au-dessus de $B$ tel que $\left(Y \mid \Delta_{Y}\right)$ soit isotrivial. 
Conjecture 12.17 Soit $(X \mid \Delta)$ une orbifolde géométrique lisse définie sur $k_{B}$, et sans quotient isotrivial.

1. $(X \mid \Delta)$ est spéciale si et seulement s'il existe une extension finie $k_{B^{\prime}} / k_{B}$ telle que $(X \mid \Delta)\left(k_{B^{\prime}}\right)$ soit Zariski dense (ie : tel que la réunion des $s(B), s \in$ $(X \mid \Delta)\left(k_{B^{\prime}}\right)$ soit Zariski dense dans $\left.X_{B^{\prime}}\right)$.

2. $(X \mid \Delta)$ est de type général si et seulement s'il existe un ouvert de Zariski dense $U$ de $X$ tel que, pour toute extension finie $k_{B^{\prime}} / k_{B}$, l'ensemble des $s \in(X \mid \Delta)\left(k_{B^{\prime}}\right)$ tels que $s\left(B^{\prime}\right)$ rencontre $U$ soit fini.

\section{Remarque 12.18}

1. La conjecture 12.17.2. est la version orbifolde de la conjecture corps de fonctions de Bombieri-Lang.

2. Le coeur montre que si $(X \mid \Delta)$ n'est pas spéciale, et si 12.17.2. est vraie, alors $(X \mid \Delta)\left(k_{B^{\prime}}\right)$ n'est Zariski dense pour aucune extension finie $k_{B^{\prime}} / k_{B}$, ce qui établit 12.17.1. dans ce cas. Plus précisément, si 12.17.2. est vraie, alors $\left(c_{(X \mid \Delta)}(X \mid \Delta)\left(k^{\prime}\right)\right) \cap U$ est fini, pour toute extension finie $k_{B^{\prime}} / k_{B}$, et pour un ouvert de Zariski non vide $U$ de $C(X \mid \Delta)$.

3. La densité potentielle des orbifoldes géométriques lisses spéciales peut être conjecturalement réduite aux cas $\kappa=0$ et $\kappa_{+}=-\infty$ par le même "dévissage" et sous les mêmes hypothèses que dans les cas précédents.

4. Voir [Ca 05] pour un cas particulier en dimension relative 2.

\subsection{Points rationnels : arithmétique}

Ce cas est analogue au précédent (aux questions usuelles d'isotrivialité près). On renvoie à [Abr06] en particulier pour une présentation et une discussion détaillée des notions présentées ici.

Si $X, \Delta=\sum_{j \in J}\left(1-1 / m_{j}\right) . D_{j}$ sont définis sur un corps de nombres $k, X$ lisse et $\operatorname{Supp}(\Delta)$ à croisements normaux, et si $\mathcal{X}, \mathcal{D}=\sum_{j \in J}\left(1-1 / m_{j}\right) . \mathcal{D}_{j}$ sont des modèles de $X, D_{j}, \forall j$ définis et de bonne réduction (ie : si la réduction de $\Delta$ reste à croisements normaux pour toute place $v \notin S$ ) sur l'anneau des entiers $\mathcal{O}_{k, S}$, on note alors $(X \mid \Delta)\left(\mathcal{O}_{k, S}\right)$ l'ensemble des points $\mathcal{O}_{k, S}$-intégraux $x \in X\left(\mathcal{O}_{k, S}\right)$ tels que pour toute place $v \notin S$ de $\mathcal{O}_{k}$, et tout $j \in J$, le nombre d'intersection arithmétique $\left(x \cdot \mathcal{D}_{j}\right)_{v}$ de $x$ avec $\mathcal{D}_{j}$ en $v$ est : soit nul, soit supérieur ou égal à $m_{j}$.

Pour tout $k, S$ fixés, les points intégraux de $(X / \Delta)$ sont essentiellement indépendants des modèles choisis : deux modèles étant choisis, ces points intégraux coincident sur un ouvert de Zariski non vide ("commun") de $X$. 
On peut introduire la notion plus restrictive de points intégraux classiques en imposant la condition que les nombres d'intersection arithmétiques $\left(x \cdot \mathcal{D}_{j}\right)_{v}$ soient divisibles par $m_{j}$. Voir [DG98] dans le cas des courbes pour les multiplicités "classiques", et [Ca05] pour les différences et motivations. On notera $(X \mid \Delta)^{*}\left(\mathcal{O}_{k, S}\right)$ l'ensemble de ces points intégraux "classiques". On a évidemment (pour un modèle fixé) : $(X \mid \Delta)^{*}\left(\mathcal{O}_{k, S}\right) \subset(X \mid \Delta)\left(\mathcal{O}_{k, S}\right)$.

Si $f:(X \mid \Delta) \rightarrow\left(Y \mid \Delta^{\prime}\right)$ est un morphisme orbifolde défini sur $k$, il induit une application naturelle $f:(X \mid \Delta)\left(\mathcal{O}_{k, S}\right) \rightarrow\left(Y \mid \Delta^{\prime}\right)\left(\mathcal{O}_{k, S}\right)$, sur des modèles sur lesquels $f$ est défini. Si ce morphisme est un morphisme orbifolde divisible, il induit aussi une application $f:(X \mid \Delta)^{*}\left(\mathcal{O}_{k, S}\right) \rightarrow\left(Y \mid \Delta^{\prime}\right)^{*}\left(\mathcal{O}_{k, S}\right)$.

Conjecture 12.19 Soit $(X \mid \Delta)$ une orbifolde géométrique lisse définie sur $k$, un corps de nombres ${ }^{44}$. On suppose fixé un modèle défini sur $\mathcal{O}_{k, S}$ comme ci-dessus.

1. $(X \mid \Delta)$ est spéciale si et seulement s'il existe une extension finie $k^{\prime} / k$ telle que $(X \mid \Delta)\left(k^{\prime}\right)$ soit Zariski dense pour un, et donc tout modèle. ("Densité potentielle")

2. $(X \mid \Delta)$ est de type général si et seulement s'il existe un ouvert de Zariski dense $U \subset X$ tel que, pour toute extension finie $k^{\prime} / k,(X \mid \Delta)\left(k^{\prime}\right) \cap U$ soit fini pour un, et donc tout modèle.

\section{Remarque 12.20}

1. La conjecture 12.19.2. n'est qu'une version orbifolde géométrique de la conjecture arithmétique de Bombieri-Lang. Remarquons que cette conjecture est ouverte même pour les courbes, pour lesquelles elle résulte cependant de la conjecture $a b c$ (voir [C05]). La version corps de fonctions complexes est cependant établie dans [Ca05]. La version "classique" peut être cependant déduite du théorème de Faltings (voir [DG98]).

2. Le coeur montre que si $(X \mid \Delta)$ n'est pas spéciale, et si 12.19.2. est vraie, alors $(X \mid \Delta)\left(k^{\prime}\right)$ n'est Zariski dense pour aucune extension finie $k^{\prime} / k$, ce qui établit 12.19.1. dans ce cas. Plus précisément, si 12.19.2. est vraie, alors $\left(c_{(X \mid \Delta)}(X \mid \Delta)\left(k^{\prime}\right)\right) \cap U$ est fini, pour toute extension finie $k^{\prime} / k$, et pour un ouvert de Zariski non vide $U$ de $C(X \mid \Delta)$.

3. La densité potentielle des orbifoldes géométriques lisses spéciales peut être conjecturalement réduite aux cas $\kappa=0$ et $\kappa_{+}=-\infty$ par le même "dévissage" que dans les cas précédents.

\footnotetext{
${ }^{44}$ On pourrait formuler cette conjecture, plus généralement, pour $k$ de type fini sur $\mathbb{Q}$.
} 
4. Voir [Ca 05] pour un énoncé de la conjecture de Mordell en version orbifolde, conséquence de la conjecture $a b c$, mais non du théorème de Faltings.

\subsection{Familles de variétés canoniquement polarisées.}

De façon vague, il est conjecturé que "l'espace des modules fin" des variétés de type général a des composantes irréductibles qui sont elles-mêmes de type général.

Dans le cas des variétés à fibré canonique ample (dont les courbes de genre $g \geq 2$ fournissent l'exemple classique), on va présenter des résultats et une conjecture plus précise, dont le cadre naturel semble être justement les notions d'orbifoldes géométriques logarithmiques spéciales et de "coeur" développées dans le présent texte.

Soit $g: V \rightarrow B$ un morphisme projectif, submersif et à fibres connexes de $V$, lisse, sur une base $B$ quasi-projective connexe. On supposera que $B=$ $\bar{B}-D$, où $\bar{B}$ est projective lisse, et $D$ un diviseur à croisements normaux de $\bar{B}$. Donc $B$ n'est autre que l'orbifolde géométrique logarithmique $(\bar{B} / D)$. On notera : $\bar{\kappa}(B):=\kappa(\bar{B} / D)$.

On suppose que $g$ est une famille de variétés canoniquement polarisées, c'est-à-dire satisfait les conditions précédentes, et que, de plus, le faisceau canonique relatif $K_{V / B}$ est ample sur toutes les fibres $V_{b}, b \in B$ de $g$.

On note alors : $\operatorname{Var}(g)$ le rang de l'application de Kodaira-Spencer $k s_{g}(b): T B_{b} \rightarrow H^{1}\left(V_{b}, T V_{b}\right)$ au point générique de $B$. Donc $\operatorname{Var}(g)=0$ si et seulement si $g$ est isotriviale (ie : ses fibres sont deux-à-deux isomorphes).

Notre conjecture ${ }^{45}$ est la suivante :

Conjecture 12.21 Si $B=(\bar{B} / D)$ est spéciale, la famille $g$ est isotriviale.

En particulier, $\operatorname{Var}(g) \leq \operatorname{dim}(C(\bar{B} / D)$, la dimension du "coeur" de la base orbifolde géométrique logarithmique de la famille considérée, dans le cas général.

En effet, pour $B$ arbitraire, la restriction de $g$ au-dessus des fibres du "coeur" $c_{B}: \bar{B} \rightarrow C(\bar{B} / D)$ de $B$ serait isotriviale, et la "variation" de g se factoriserait par $C(\bar{B} / D)$ (ie : au point générique $b$ de $B$, l'application de Kodaira-Spencer $k s_{g}(b)$ s'annule sur l'espace tangent à la fibre de $c_{B}$ en $b$ ).

\footnotetext{
${ }^{45}$ On pourrait la formuler, plus généralement, lorsque les fibres $V_{b}$ ont un fibré canonique semi-ample, ou même nef.
} 
Cette conjecture généralise et renforce en les précisant considérablement les 3 conjectures antérieures $A, B, C$ suivantes :

- A. (Viehweg, voir [V-Z02]) : si $\operatorname{Var}(g)=\operatorname{dim}(B)$, alors $B$ est de type général (ie : $\bar{\kappa}(B):=\operatorname{dim}(B))$. En effet, cette égalité entrainerait alors : $\operatorname{dim}(B)=\operatorname{Var}(g) \leq \operatorname{dim}(C(\bar{B} / D) \leq \operatorname{dim}(B)$, or $\operatorname{dim}(B)=\operatorname{dim}(C(\bar{B} / D)$ si et seulement si $B$ est de type log-général.

- B. $([$ Ke-Kov06] Si $\overline{\kappa(B)}=0$, alors $\operatorname{Var}(g)=0$ (c'est-à-dire que $g$ est isotriviale). En effet, la condition $\overline{\kappa(B)}=0$ entraine que $B$ est Log-spéciale.

- C. ([Ke-Kov06] Si $\bar{\kappa}(B)=-\infty$, alors $\operatorname{Var}(g) \leq(\operatorname{dim}(B)-1)$. En effet, si $\bar{\kappa}(B)=-\infty, B$ n'est pas de type Log-général. Et $\operatorname{dim}(C(\bar{B} / D) \leq(n-1)$.

- La décompostion conditionnelle du coeur montre que les deux cas cruciaux dans lesquels la conjecture devrait être d'abord établie sont les cas où $\overline{\kappa_{+}}(B)=-\infty$ (en particulier lorsque $(\bar{B} / D)$ est Fano), et lorsque $\bar{\kappa}(B)=0$ (en particulier lorsque $c_{1}(\bar{B} / D)=0$ ). Cependant, la conjecture 12.21 traite aussi les nombreux cas dans lesquels $(\bar{B} / D)$ "fibre" de manière itérée (au sens orbifode) avec des fibres de l'un des deux types précédents. Le premier cas non traité par les conjectures $\mathrm{A}, \mathrm{B}, \mathrm{C}$ ci-dessus étant celui dans lequel $\bar{\kappa}(B)=1$ et $\operatorname{dim}(B)=2$. Il est résolu ci-dessous, à titre d'exemple.

La conjecture 12.21 est démontrée lorsque $\operatorname{dim}(B)=1$ ( [Kov96], [Kov00], généralisant le cas classique dans lequel les $V_{b}$ sont des courbes de genre $g \geq 2$ ).

Lorsque $\operatorname{dim}(B)=2$, les conjectures $\mathrm{A}$, B et $\mathrm{C}$ ci-dessus sont démontrées dans $[$ Ke-Kov06].

Cependant, même lorsque $\operatorname{dim}(B)=2$, il existe de nombreux cas ( $B$ spéciale avec $\bar{\kappa}(B)=1$ ou $-\infty$ ) dans lesquels la conjecture 12.21 renforce les conjectures A,B,C (la conclusion étant " $g$ isotriviale", et non : "Var $(g) \leq 1$ ").

Théorème 12.22 Soit $g: V \rightarrow B$ une famille de variétés canoniquement polarisées (dans le sens ci-dessus). On suppose que $\operatorname{dim}(B)=2$, et que $B$ est spéciale. Alors g est isotriviale.

Démonstration : Tout comme dans [Ke-Ko06], la démonstration résulte essentiellement du résultat suivant de $[\mathrm{V}-\mathrm{Z}]$ :

Théorème 12.23 Soit $g: V \rightarrow B$ une famille de variétés canoniquement polarisées. Il existe alors un entier $N>0$ et un sous-fibré $L$ de rang 1 de $S_{N, 1}(B):=S_{N, 1}(\bar{B} / D)=\operatorname{Sym}^{N}\left(\Omega_{\bar{B}}^{1}(\log (D))\right.$ tel que $\kappa(\bar{B}, L) \geq \operatorname{Var}(g)$.

Le théorème 12.22 résulte alors du : 
Lemme 12.24 Soit $B=(\bar{B} / D)$ une orbifolde géométrique lisse, projective et logarithmique spéciale de dimension 2. Pour tout $N>0$, et pour tout sous-fibré $L$ de rang 1 de $S_{N, 1}(B)$, on a alors : $\kappa(\bar{B}, L) \leq 0$.

Démonstration : Nous traitons successivement les cas $\bar{\kappa}(B)=0, \bar{\kappa}(B)=$ $-\infty$, et $\bar{\kappa}(B)=1$. Les deux premiers cas étant déjà essentiellement connus.

- Lorsque $\bar{\kappa}(B)=0$, le résultat est, en fait, établi dans [Ke-Kov06]. Il nous reste à traiter les cas $\bar{\kappa}(B)=-\infty$ et $\bar{\kappa}(B)=1$.

- Lorsque $\bar{\kappa}(B)=-\infty$, il résulte de [K-McK99] que $\bar{B}$ est recouverte par des courbes rationnelles rencontrant $D$ en au plus un point (unibranche). Si le point générique de $\bar{B}$ est contenu dans 2 telles courbes distinctes (au moins), alors $(\bar{B} / D)$ est spéciale, et $g$ isotriviale (par [Kov00], par exemple).

Sinon, il existe une fibration $f:(\bar{B} / D) \rightarrow C$ dont la fibre générique est l'une des courbes rationnelles précédentes. Et $D=D^{h}+D^{v}$ est alors réunion de $D^{h}$, soit vide, soit une section de $f$, tandis que $D^{v}$ est effectif, contenu dans une réunion finie de fibres de $f$. Soit $F_{c} \cong \mathbb{P}^{1}$ une fibre générique lisse de $f$. La restriction de $\Omega_{(\bar{B} / D)}^{1}$ à $F=F_{c}, c \in C$ est une extension de $\mathcal{O}_{F}(-d)$ par $\mathcal{O}_{F}=f^{*}\left(T_{C, c}^{*}\right)$, avec $d=1$ si $D^{h} \neq 0$, et $d=2$ sinon. Donc, pour tout $N>0$, les sections de $S_{N, 1}(B)$ sur $F$ forment un espace vectoriel complexe de dimension 1 engendré par $f^{*}\left(\left(T_{C, c}^{*}\right)^{\otimes N}\right)$.

Les sections de $S_{N, 1}(B)$ sont donc de la forme : $f^{*}\left(N . K_{C}+\Delta_{C}\right)$, pour $\Delta_{C}$ un diviseur effectif sur $C$. Par la proposition 4.6, et l'exemple $3.9, \Delta_{C} \leq$ $N . \Delta(f, D)$.

Si $S_{N, 1}(B)$ admet deux sections non nulles $s, t$ telles que $t=u . s$, pour $u$ méromorphe sur $\bar{B}$, c'est donc que $\kappa(C \mid \Delta(f, D))=1$, et $(\bar{B} / D)=B$ n'est donc pas spéciale.

- Supposons donc désormais que $\bar{\kappa}(B)=1$. Soit $f:(\bar{B} / D) \rightarrow C$ la fibration de Moishezon-Iitaka. Soit $F:=F_{c}, c \in C$ une fibre générique lisse de $f$. Alors $F$ est soit elliptique, soit rationnelle, et $D=D^{h}+D^{v}$ est réunion de $D^{v}$ effectif, contenu dans une réunion finie de fibres de $f$, tandis que $D^{h}$ est soit vide (si $F$ est elliptique), soit réduit de degré 2 sur $C$.

On note $\pi: \mathbb{P}\left(\Omega_{\bar{B}}^{1}\right) \rightarrow \bar{B}$ le projectifié (des droites) du fibré cotangent de $\bar{B}$. Soit $s$ une section non nulle de $S_{N, 1}(B)$, et $\widetilde{B} \subset \mathbb{P}\left(\Omega_{\bar{B}}^{1}\right)$ le projectifié (non nécessairement réduit) du sous-faisceau tautologique engendré par $s$. La projection $\pi$ fait de $\widetilde{B} \rightarrow \bar{B}$ un revêtement de degré $N$. Puisque la restriction de $\Omega_{(\bar{B} / D)}^{1}$ à $F=F_{c}, c \in C$ est une extension de $\mathcal{O}_{F}=\Omega_{\left(F / D_{F}\right)}^{1}$ par $\mathcal{O}_{F}=$ $f^{*}\left(T_{C, c}^{*}\right)$, il existe un revêtement ramifié $h: \widetilde{C} \rightarrow C$ tel que $\widetilde{B}$ soit déduit de 
$f: \bar{B} \rightarrow C$ par le changement de base $h: \widetilde{C} \rightarrow C$. De plus, par construction, $\pi^{*}(s)=s_{1} \otimes s_{2} \otimes \cdots \otimes s_{N}$, avec $s_{j} \in H^{0}\left(\widetilde{B}, \Omega_{(\widetilde{B} / \widetilde{D})}^{1}\right)$, avec $\widetilde{D}=\pi^{-1}(D)$.

Supposons maintenant qu'il existe une seconde section $t$ de $S_{N, 1}(B)$ telle que $t=u . s, u$ étant méromorphe non constante sur $\bar{B}$. Par ce qui précède, $u=f^{*}(v)$, pour $v$ méromorphe non constante sur $C$, et $\pi^{*}(t)=t_{1} \otimes t_{2} \otimes$ $\cdots \otimes t_{N}$, avec $t_{j} \in H^{0}\left(\widetilde{B}, \Omega_{(\widetilde{B} / \widetilde{D})}^{1}\right)$, et pour $j=1, \ldots, N, t_{j}=v_{j}^{\prime} \cdot s_{j}$, avec $v_{j}^{\prime}$ méromorphe sur $\widetilde{C}$.

Il en résulte que $s$ et $t$ sont des sections méromorphes de $f^{*}\left(N . K_{C}\right)$. On en déduit, comme dans le cas où $\bar{\kappa}(B)=-\infty$, que la base orbifolde de $f$ est de type général, et donc que $B$ n'est pas spéciale.

Remarque 12.25 Lorsque la fibration de Moishezon-Iitaka $f:(\bar{B} / D) \rightarrow C$ n'est pas isotriviale (et $F$ est donc elliptique), on peut démontrer plus directement que $L \subset f^{*}\left(N . K_{C}\right)$ au-dessus du point générique $c$ de $C$ : puisque l'application de Kodaira-Spencer $k s_{f}(c)$ n'est pas nulle en $c$, les classes successives des extensions déduites de la filtration naturelle de quotients $\left(T F^{*}\right)^{\otimes j} \otimes\left(f^{*}\left(T C_{c}\right)\right)^{\otimes(N-j)}$ de $S y m^{N}\left(\Omega_{\bar{B}}^{1}\right) \mid F$ comme extension de $T F^{*}$ par $f^{*}\left(T C_{c}\right)$ sont des multiples entiers non nuls de $k s_{f}(c)$. Les sections de $\operatorname{Sym}^{N}\left(\Omega_{\bar{B}}^{1}\right) \mid F$ se réduisent donc à celles de $\left(f^{*}\left(T C_{c}\right)\right)^{\otimes N}=f^{*}\left(K_{C, c}^{\otimes N}\right)$.

Question 12.26 Soit $(B \mid \Delta)$ une orbifolde géométrique lisse spéciale avec $B \in \mathcal{C}$. Peut-il exister un sous-fibré $L \subset S_{N, 1}(B \mid \Delta)$ de rang 1 tel que $\kappa(B, L)>0$ ? Une réponse positive (qu'il est optimiste d'attendre en toute dimension : l'argument précédent utilise une propriété très spécifique à la dimension 2 : la décomposabilité des produits symétriques de 1-formes) impliquerait la conjecture 12.21.

\subsection{Formes différentielles}

Soit $(X \mid \Delta)$ une orbifolde géométrique lisse avec $X \in \mathcal{C}$.

Soit $q \geq 0$ un entier, et $\Omega^{q}(X \mid \Delta):=\oplus_{N \geq 0} H^{0}\left(X, S_{N, q}\right)$ l'algèbre des $q$ formes différentielles symétriques sur $(X \mid \Delta)$.

Pour $x \in X, x \notin \operatorname{Supp}(\Delta)$, on a une application naturelle d'évaluation en $x: e v_{x}^{q}: \Omega^{q}(X \mid \Delta) \rightarrow \oplus_{N \geq 0} \operatorname{Sym}^{N}\left(\Omega_{X, x}^{q}\right)$.

\section{Conjecture 12.27}

1. Si $\kappa_{+}(X \mid \Delta)=-\infty$, alors $\Omega^{q}(X \mid \Delta)=\mathbb{C}, \forall q \geq 0$.

2. Si $\kappa(X \mid \Delta)=0$, alors ev $x_{x}^{q}$ est injective pour $x \in X$ générique. 


\section{Remarque $\mathbf{1 2 . 2 8}$}

0 . La conjecture précédente entraine en particulier que : si $L \subset \Omega_{X}^{p}, p>0$, on a : $\kappa((X \mid \Delta), L) \leq 0$ si $\kappa(X \mid \Delta)=0$, et : $\kappa((X \mid \Delta), L)=-\infty$ si $\kappa_{+}(X \mid \Delta)=$ $-\infty$.

1. La conjecture 12.27.1 est vraie pour les variétés rationnellement connexes (avec $\Delta=0$ ). Elle l'est aussi pour les orbifoldes rationnellement connexes (au sens orbifolde). Elle doit pouvoir être établie au moins dans le cas des multiplicités entières et finies à l'aide de métriques orbifoldes à courbure de Ricci positive.

2. La conjecture 12.27.2 est vraie pour les variétés Kählériennes compactes avec $c_{1}(X)=0$ (et $\Delta=0$ ), par la solution de la conjecture de Calabi sur l'existence de métriques de Kähler Ricci-plates et le parallélisme des $q$-formes symétriques qui en résulte. La conjecture de K. Ueno $\left(h^{0}\left(X, \Omega_{X}^{q}\right) \leq \frac{n !}{q !(n-q) !}\right.$ si $\kappa(X)=0$, avec $n:=\operatorname{dim}(X))$ est un cas particulier de 12.27. Elle doit pouvoir être établie au moins dans le cas des orbifoldes géométrique à multiplicités entières et finies à l'aide de métriques orbifoldes à courbure de Ricci nulle.

3. Une conjecture sur l'algèbre $\Omega^{q}(X \mid \Delta)$ lorsque $(X \mid \Delta)$ est spéciale semble plus difficile à formuler, le dévissage exposé au $\S 10.5$ ne fournissant pas de structure simple apparente, même en admettant 12.27.

\section{BIBLIOGRAPHIE}

[Abr 07]D. Abramovich. Birational geometry for number theorists. math. AG/0701105v2

[Ba75] D. Barlet. Espace analytique réduit des cycles analytiques complexes compacts d'un espace analytique de dimension finie. LNM 482 (1975), $1-158$.

[BCHM06]C.Birkar-P.Cascini-C.Hacon-J. McKernan. Existence of minimal models for varieties of log general type. math. AG/0610203

[Bo79] F. Bogomolov. Holomorphic tensors and vector bundles on projective varieties. Math. Ussr Izv. 13 (1979), 499-555.

[Br 08] A. Broustet. Communication orale (Novembre 2008).

[Ca 92] F. Campana. Connexité rationnelle des variétés de Fano. Ann. Sc. ENS. 25 (1992), 539-545.

[Ca93] F.Campana.Remarques sur les groupes de kähler nilpotents. Ann. Sc. ENS 28(1993), 307-316. 
[Ca94] F.Campana.Remarques sur le revêtement universel des variétés kählériennes compactes. Bull. SMF 122(1994), 255-284.

[Ca98] F.Campana. $\mathcal{G}$-connectedness of compact kähler manifolds. Cont. Math. 241(1999), 85-97.

[Ca01]F.Campana. Special varieties and classification theory. math.AG/0110151.

[Ca04]F.Campana. Orbifolds, special varieties and classification theory. Ann.Inst. Fourier. 54 (2004), 499-665.

[Ca05] F. Campana. Fibres multiples sur les surfaces. Man. Math. 117(2005), 429-461.

[C-P05] F.Campana-M.Paun. Variétés faiblement spéciales à courbes entières dégénérées. math.AG/0512124. A paraitre aux Comp. Math.

[C-Pe 06] F. Campana-T. Peternell. Geometric stability of the cotangent bundle and the universal cover of a projective manifold. Disponible sur arXiv.

[C-W05]F.Campana-J. Winkelmann. A Brody theorem for orbifolds. preprint arXiv.

[Cla06]B.Claudon. Invariance for multiples of the twisted canonical bundle. Ann. Inst. Fourier 57 (2007), 289-300. (Aussi : Math. AG/0511736).

[Cla08]B.Claudon. Gamma-reduction for smooth orbifolds. arXiv : 0801.2894 .

[DG98]H. Darmon-A. Granville. On the equations $z^{m}=F(x, y)$ and $A x^{p}+B y^{q}=C z^{r}$. Bull. London Math. Soc. 27(19995), 513-543.

[De74] P. Deligne. Théorie de Hodge II. Publ. IHES 40 (1972), 5-57.

[Delz06]T. Delzant. L'invariant de Bieri Neuman strebel des groupes fondamentaux des variétés kählériennes. math.DG/0603038

[Fuj78] T.Fujita. On Kähler fibre spaces over curves. J. Math. Soc. Jap. 30 (1978), 779-794.

[GHS03] T.Graber-J.Harris-J.Starr. Families of rationally connected varieties. J. Amer. Math. Soc. 16(2003), 57-67.

[G-K-K08] D. Greb, S. Kebekus, S. Kovàcs. Extension theorems for differential forms and Bogomolov-Sommese vanishing on Log-canonical varieties. arXiv : 0808.3647.

[Kaw81] Y.Kawamata. Characterisation of Abelian Varieties. Comp. Math. (1981), 253-276.

[Kaw98] Y.Kawamata. Subadjunction of log-canonical divisors II. Amer. J. Math. 120 (1998), 893-899.

[Ke-Kov06] S. Kebekus-S. Kovàcs. Families of canonically polarized varieties over surfaces. math.arXiv. To appear in Inv. Math. 
[K-McK99] S. Keel-J. McKernan. Rational curves on quasi-projective surfaces. Memoirs of the AMS 669 (1999).

[Ko93]J. Kollár. Shafarevitch maps and plurigenera of algebraic varieties. Inv. Math. 113 (1993), 177-215.

[KoMiMo92]J.Kollár-Y.Miyaoka-S.Mori. Rationally connected varieties. J. Alg. Geom. 1 (1992), 429-448.

[Kov96] S.Kovàcs. Smooth families over rational and elliptic curves. JAG 5 (1996), , 369-385.

[Kov00] S.Kovàcs. Algebraic hyperbolicity of fine moduli spaces. JAG 9 (2000), 169-174.

[La86] S. Lang. Hyperbolic and Diophantine Analysis. Bull. AMS 14(1986), 159-205.

[Lieb78]D.Lieberman. Compactness of the Chow Scheme. LNM 670 (1975), 140-186.

[Miy 87] Y. Miyaoka. Deformation of a morphism along a foliation. Proc. Symp. Pure Math. vol. 46, 245-268 (1987)

[Mi-Mo 86] Y. Miyaoka-S. Mori. A numerical criterion for uniruledness. Ann. Math. 124 (1986), 65-69.

[N87]M.Namba. Branched coverings and algebraic functions. Pitman research Notes in Mathematics series 161. Longman Scientific and Technical (1987).

[Pa05]M.Paun.Siu's invariance of plurigenera : a one-tower proof . A paraitre au J. Diff.Geom.

[R72] M. Raynaud. Flat modules in algebraic geometry. Comp. Math. 24 (1972), 11-31.

[R08] E. Rousseau. Hyperbolicity of geometric orbifolds. arXiv :08091356.

[Sak 74] F. Sakai. Degeneracy of holomorphic maps with ramifications. Inv. Math. 28 (1974), 213-229.

[SB 92] N. Shepherd-Barron. Miyaoka's theorem on the semi-negativity of $T_{X}$. Astérisque 211, 103-114 (1992)

[Siu02]Y.T.Siu. Extension of twisted pluricanonical sections with plurisubharmonic weights. Complex Geometry (Göttingen 2000),223-277, Springer (2002).

[U75] K. Ueno. Classification theory of complex analytic manifolds. LNM 439 (1975)

[Vie83] E. Viehweg. Weak positivity and the additivity $\mathrm{f}$ the Kodaira dimension for certain fibre spaces. Ad. Studies in Pure Math. 1 (1983), 329353. 
[V-Z02] E. Viehweg-K. Zuo. Base spaces of non-isotrivial families of smooth minimal models. Complex geometry (Göttingen 2000), 279-328. Springer Verlag 2002.

F. Campana

Département de Mathématiques

Université Nancy 1

BP 239

F-54506 Vandoeuvre-lès-Nancy Cedex

campana@iecn.u-nancy.fr 